\title{
ERKLÄRUNG VON RECHTSCHREIBLEISTUNGEN DURCH PHONOLOGISCHE UND AUDITIV-SENSORISCHE INFORMATIONS- VERARBEITUNGSKOMPETENZEN: EINE UNTERSUCHUNG MIT LESE-/RECHTSCHREIBGESTÖRTEN KINDERN
}

Dissertation zur Erlangung des Doktorgrades der Mathematisch-Naturwissenschaftlichen Fakultäten der Georg-August-Universität zu Göttingen.

vorgelegt von

Anette Ulrich-Brink

aus Nürnberg

Göttingen, 2004 
D 7

Referent: $\quad$ Prof. Dr. M. Hasselhorn

Korreferent: Prof. Dr. A. Rothenberger

Tag der mündlichen Prüfung: 01. Juli 2004 
FÜR MEINEN VATER 


\section{INHALTSVERZEICHNIS}

1. EINLEITUNG.

2. THEORIE

2.1. DAS STÖRUNGSBILD "LESE-/RECHTSCHREIBSCHWÄCHE“"

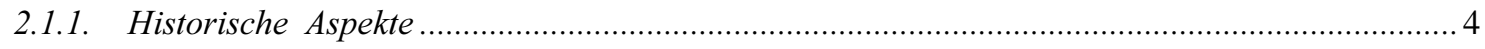

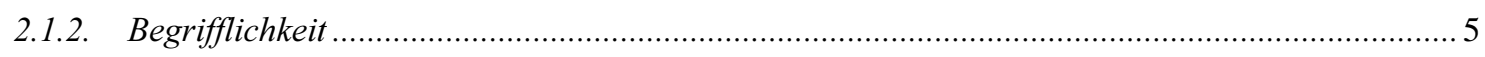

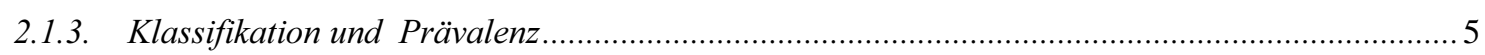

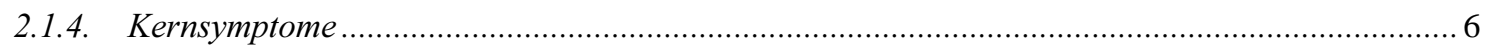

2.2. URSACHENBEREICHE DER LESE-/RECHTSCHREIBSCHWÄCHE

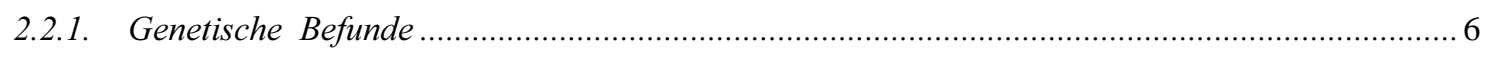

2.2.2. Strukturelle und neurologische Befunde .............................................................................. 7

2.2.3. Phonologische Informationsverarbeitung ........................................................................ 9

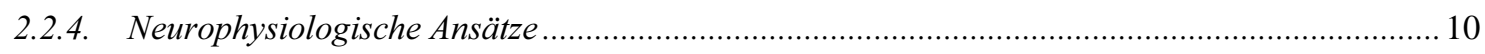

\subsection{KOGNITIONSPSYCHOLOGISCHE KONZEPTE UND METHODEN}

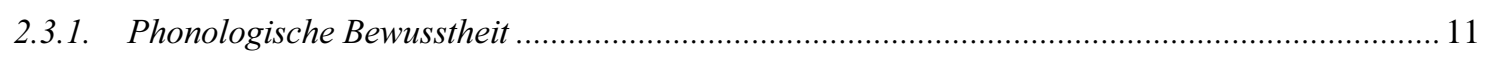

2.3.2. Abruf phonologischer Codes aus dem Langzeitgedächtnis ....................................................... 13

2.3.3. Das Arbeitsgedächtnis (nach Baddeley \& Hitch, 1974) .................................................... 13

2.3.3.1. Das Arbeitsgedächtnismodell: Überblick ..................................................................................... 14

2.3.3.2. Architektur der phonologischen Schleife und Interaktionen innerhalb des Gedächtnisses ................... 14

2.3.3.3. Funktionsmerkmale der phonologischen Schleife und Befunde defizitärer Verarbeitung bei lese/rechtschreibschwachen Personen............................................................................................... 17

2.3.3.4. Repräsentationsformat innerhalb der phonologischen Schleife ......................................................... 20

2.3.3.5. Relevanz des Arbeitsgedächtnisses für den Schriftspracherwerb ........................................................ 21

\subsection{NEUROPHYSIOLOGISCHE KONZEPTE UND METHODEN}

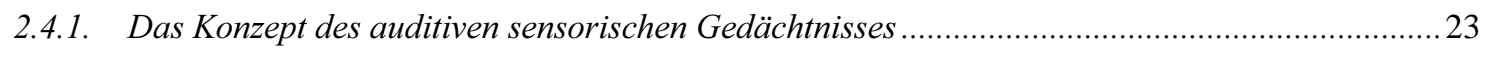

2.4.2. Untersuchungsmethoden zum auditiven sensorischen Gedächtnis...........................................23

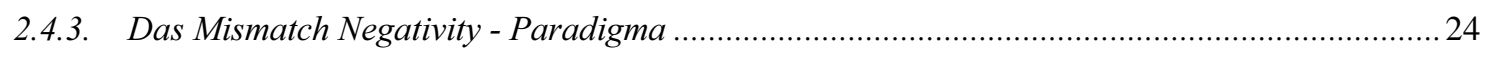

2.4.4. Befunde zum auditiven sensorischen Gedächtnis im Kontext von Lese-/Rechtschreibschwäche 29

2.4.4.1. Defizit der rasch temporalen Verarbeitung ........................................................................................... 29

2.4.4.2. Generelles auditorisches Verarbeitungsdefizit............................................................................ 32

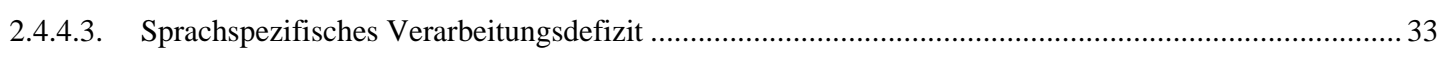


3. EIGENE FRAGESTELLUNG

3.1. PhONOLOGISCHE VERARBEITUNGSDEFIZITE VON KINDERN MIT LESE-/RECHTSCHREIBSCHWÄCHE (KOGNITIONSPSYCHOLOGISCHE EBENE)....

3.2. SENSORISCH AUDITORISCHE VERARBEITUNGSDEFIZITE VON KINDERN MIT LESE-/RECHTSCHREIBSCHWÄCHE (NEUROPHYSIOLOGISCHE EBENE)

3.3. ZUSAMMENHANG ZWISCHEN PHONOLOGISCHEN UND AUDITIV-SENSORISCHEN

VERARBEITUNGSDEFIZITEN

4. METHODE

4.1. DESIGN. 44

\subsection{UNABHÄNGIGE VARIABLEN}

4.2.1. Definition der Gruppenzugehörigkeit 44

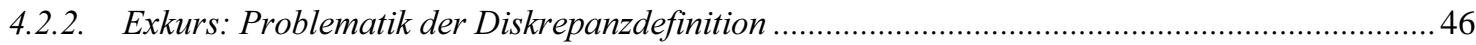

4.2.3. Instrumente zur Erhebung der Gruppenzugehörigkeit ..........................................................47

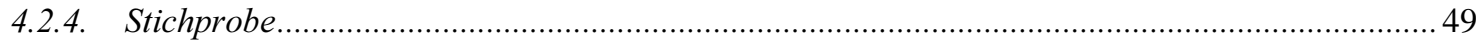

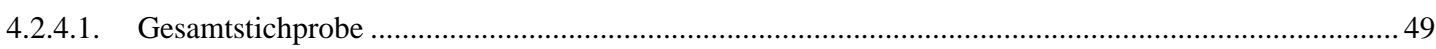

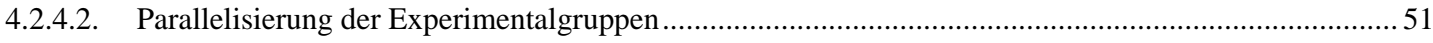

4.2.4.3. Lese-/Rechtschreibparameter der Experimentalgruppen .................................................................... 53

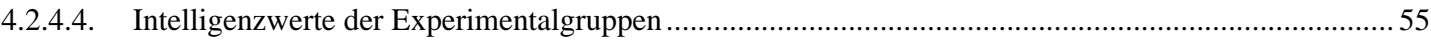

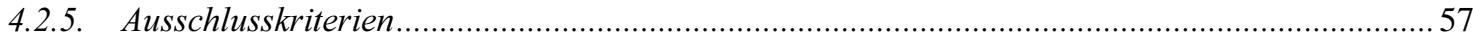

4.2.6. Instrumente zur Erfassung potentieller Ausschlusskriterien .............................................57

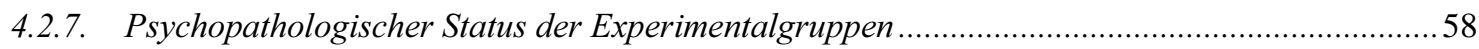

\subsection{ABHÄNGIGE VARIABLEN}

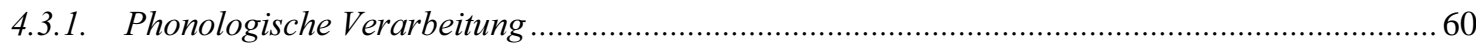

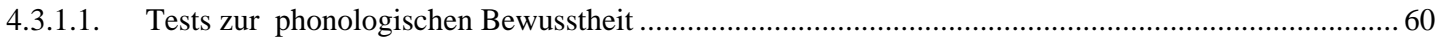

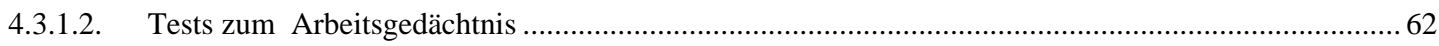

4.3.1.3. Tests zum Abruf aus dem Langzeitgedächtnis.................................................................................. 63

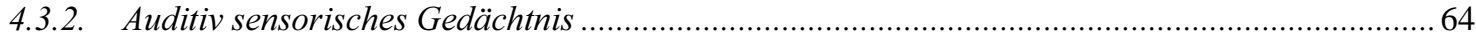

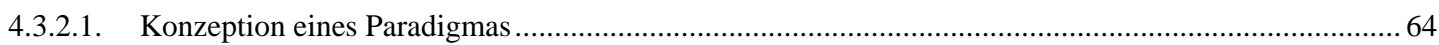

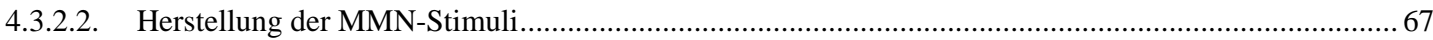

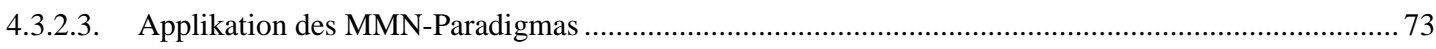

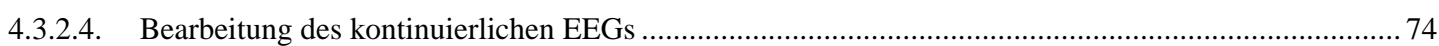

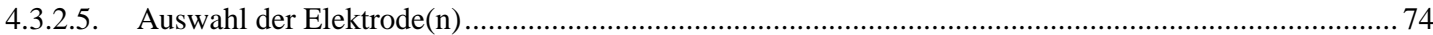

4.3.2.6. Segmentierung der Mismatch Negativity - Kurven ....................................................................... 75

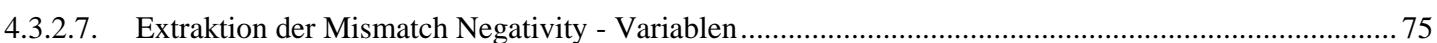


4.4. KONZEPTE ZUR UNTERSUCHUNG EINES ZUSAMMENHANGS ZWISCHEN KOGNITIONSPSYCHOLOGISCHEN UND AUDITIV SENSORISCHEN VERARBEITUNGSDEFIZITEN............................................76

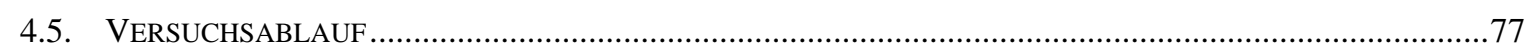

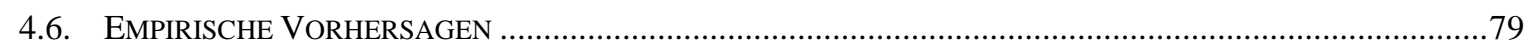

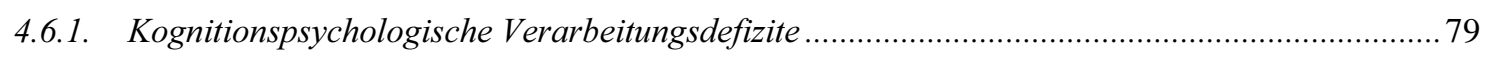

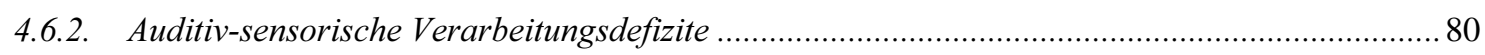

4.5.5. Zusammenhang zwischen phonologischen und auditiv sensorischen Verarbeitungsdefiziten......81

\section{ERGEBNISSE.}

\subsection{GRUPPENUNTERSCHIEDE DER PHONOLOGISCHEN VERARBEITUNG}

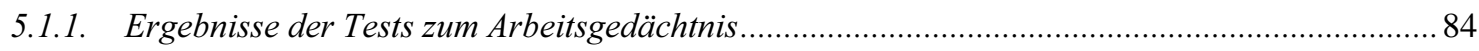

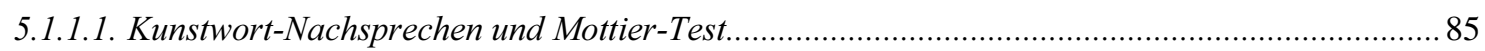

5.1.1.1.1. Ergebnisse des Kunstwort-Nachsprechens............................................................................... 85

5.1.1.1.2. Zusammenfassung und Beurteilung hinsichtlich der empirischen Vorhersage 1.a ....................... 88

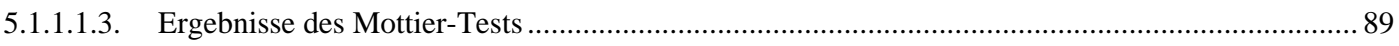

5.1.1.1.4. Zusammenfassung und Beurteilung hinsichtlich der empirischen Vorhersage 1.a ....................... 91

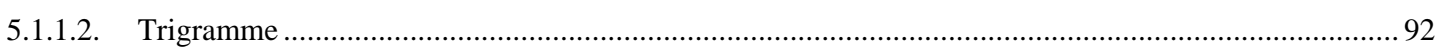

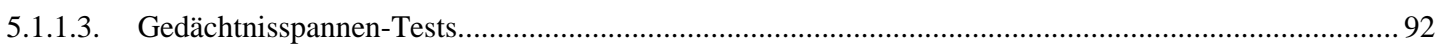

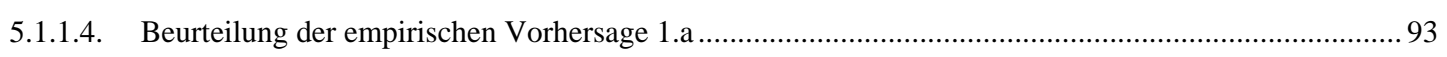

5.1.2. Ergebnisse der Tests zur phonologischen Bewusstheit....................................................... 93

5.1.3. Ergebnisse der Tests zum Abruf aus dem Langzeitgedächtnis ...................................................94

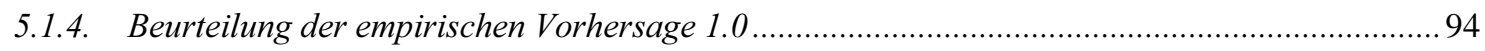

\subsection{GRUPPENUNTERSCHIEDE DER AUDITIV SENSORISCHEN VERARBEITUNG}

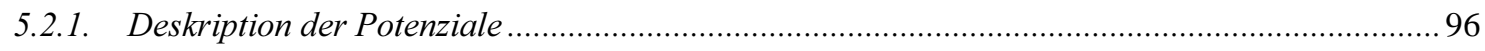

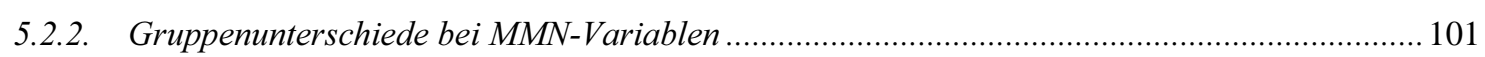

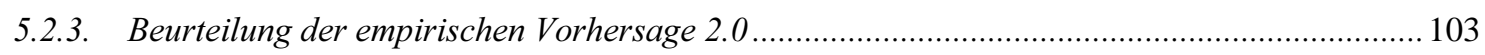

5.2.4. Zur Spezifikation des auditiven sensorischen Defizits ........................................................... 104

\subsection{ZUSAMMENHANG ZWISCHEN PHONOLOGISCHER UND AUDITIV SENSORISCHER VERARBEITUNG}

5.3.1. Bereitstellung geeigneter Variablen für die weiteren Analysen .............................................. 106

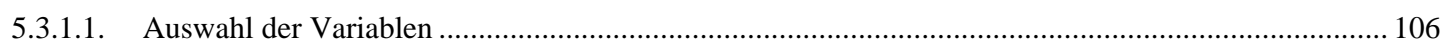

5.3.1.2. Verifikation der Realisation der Konstrukte ................................................................................ 108

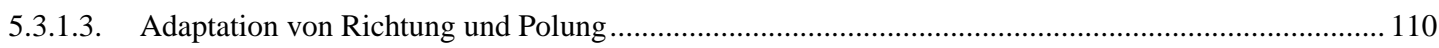


5.3.2. Erklärungskraft der aggregierten abhängigen Variablen für die Rechtschreibleistung 111

5.3.2.1. Aggregation der abhängigen Variablen und Bestimmung der Erklärungskraft der Aggregate

5.3.2.2. Struktur der Varianzen der kognitionspsychologischen Konstrukte . 115

5.3.2.3. Implementierung der EEG-Variablen in das Varianzschema der kognitionspsychologischen Konstrukte

5.3.2.3.1. Regression der einzelnen EEG-Variablen ....................................................................................... 120

5.3.2.3.2. Gegenseitige Überschneidung der EEG-Varianzen ............................................................... 121

5.3.2.3.3. Additive Varianzbereiche bezüglich der kognitionspsychologischen Konstrukte....................... 122

5.3.2.3.4. Zusammenfügen der EEG-Varianzen zum Varianzschema der kognitionspsychologischen Konstrukte.

5.3.2.4. Beurteilung der empirischen Vorhersage 3a und Zusammenfassung der Untersuchungen zum Erklärungspotenzial der Konstrukte / Ebenen.

5.3.3. Modellierung des Zusammenhangs phonologischer und auditiv sensorischer Verarbeitungs-

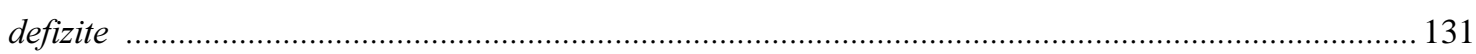

5.3.3.1. Prüfung der Modellstruktur gemäß der empirischen Vorhersage 3b .................................................. 134

5.3.3.1.1. Struktur der kognitionspsychologischen Konstrukte............................................................ 134

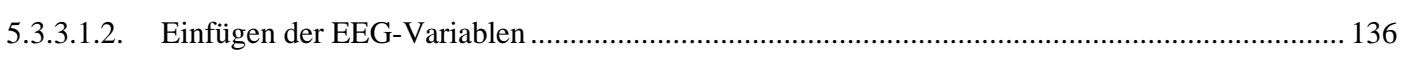

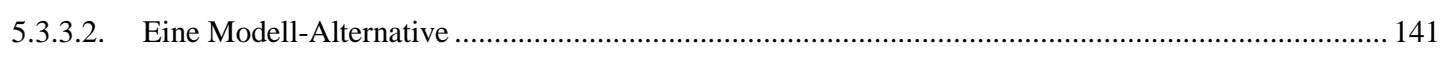

5.3.3.2.1. Struktur der kognitionspsychologischen Konstrukte............................................................. 141

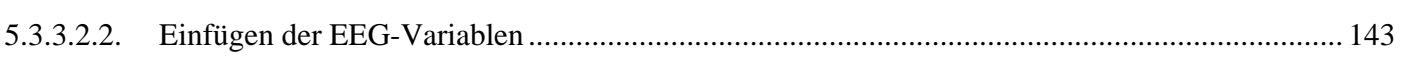

5.3.3.3. Beurteilung der empirischen Vorhersage $3 \mathrm{~b}$ und Zusammenfassung der Modell-Prüfung .................. 146

5.3.4. Zusammenfassung der Analysen des Zusammenhangs auditiv sensorischer und phonologischer Verarbeitungsdefizite bei lese-/rechtschreibschwachen Kindern 147

6. DISKUSSION.

\subsection{DISKUSSION DER BEFUNDE ZUR PHONOLOGISCHEN VERARBEITUNG}

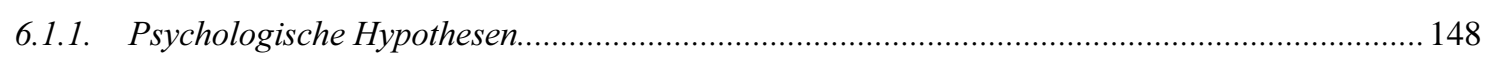

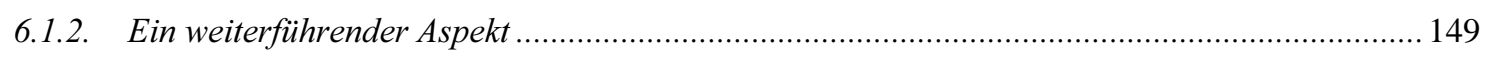

\subsection{DISKUSSION DER BEFUNDE ZUR AUDITIV SENSORISCHEN VERARBEITUNG}

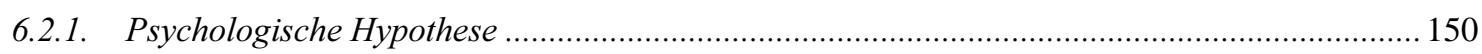

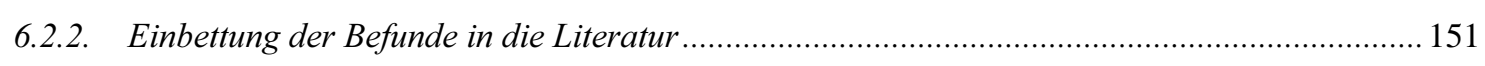

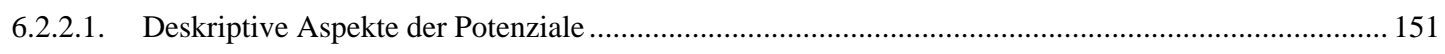

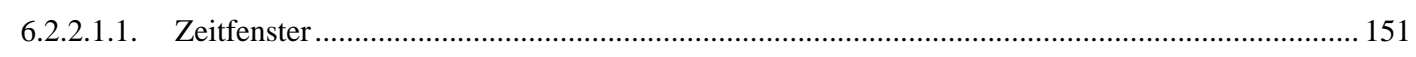

6.2.2.1.2. Form der Potenziale ……..................................................................................................... 152 


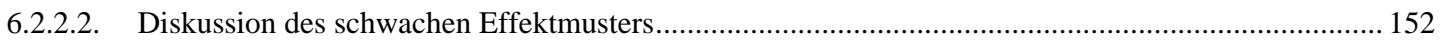

6.2.2.2.1. Vergleich mit Effektstärken aus der Literatur ......................................................................... 152

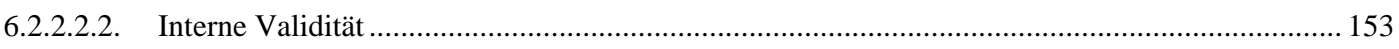

6.2.2.2.3. Variablenvalidität: Diskriminative Aufgabenschwierigkeit ...................................................... 154

\subsubsection{Diskussion der Spezifikation des auditiv sensorischen Verarbeitungsdefizits lese-/recht-}

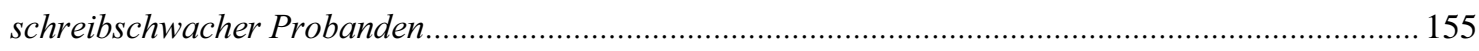

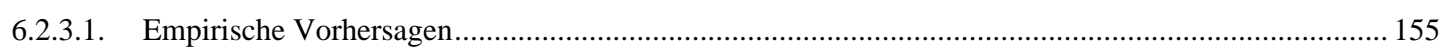

6.2.3.1.1. Ablehnung eines generellen Verarbeitungsdefizits .............................................................. 156

6.2.3.1.2. Ablehnung eines spezifisch sprachlichen Verarbeitungsdefizits............................................... 157

6.2.3.1.3. Annahme eines Defizits der rasch temporalen Verarbeitung .................................................... 158

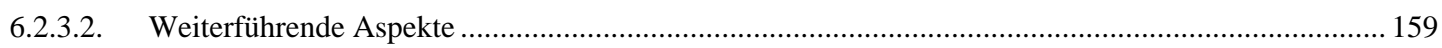

6.2.3.2.1. Spektrale Komplexität und „Sprachlichkeit“ der Sprache.......................................................... 159

6.2.3.2.2. Überlegungen zu einem verlängerten MMN-Integrationsfenster .............................................. 160

6.2.3.3. Zusammenfassung der Diskussion zur Spezifikation eines auditiv sensorischen Defizits .................. 161

\subsection{ANALYSE DES ZUSAMMENHANGS PHONOLOGISCHER UND AUDITIV SENSORISCHER}

VERARBEITUNGSDEFIZITE BEI LESE-/RECHTSCHREIBSCHWACHEN KINDERN

6.3.1. Diskussion der Analysen zum Vorliegen eines Zusammenhangs............................................. 163

6.3.1.1. Psychologische Hypothese 3a............................................................................................... 163

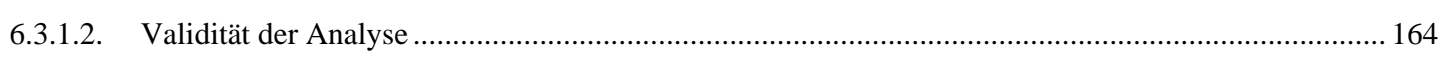

6.3.1.3. Zusammenhänge zwischen Konstrukten und Ebenen .......................................................................... 164

6.3.1.3.1. Zusammenhang zwischen den kognitionspsychologischen Konstrukten und den

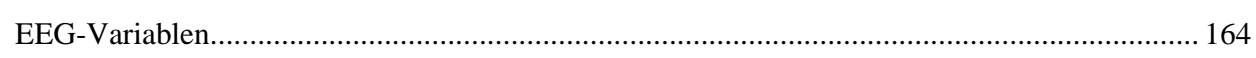

6.3.1.3.2. Zusammenhang unter den kognitionspsychologischen Konstrukten.......................................... 165

6.3.1.3.3. Zusammenhang unter den EEG-Variablen............................................................................. 165

6.3.1.4. Anmerkungen zu einzelnen Konstrukten ..................................................................................... 166

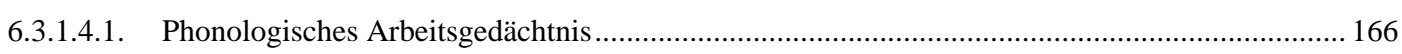

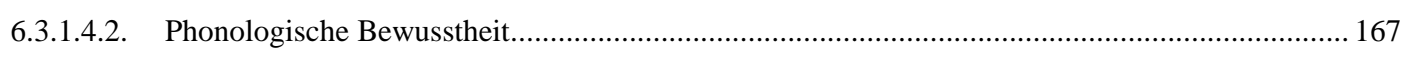

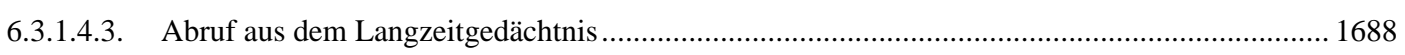

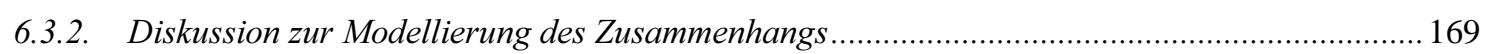

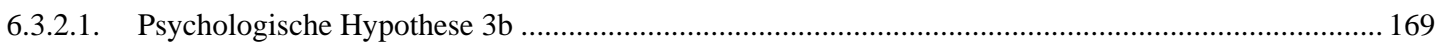

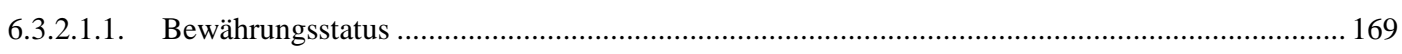

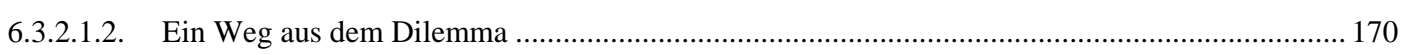

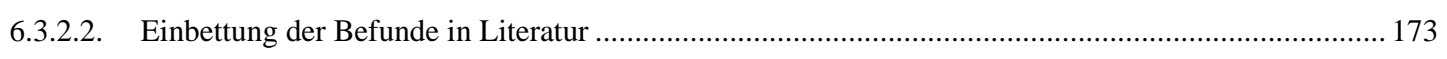

6.3.2.2.1. Zur Vorstellung verzerrter Repräsentationen an der Basis der Störung .................................... 173

6.3.2.2.2. Vergleich mit dem LISREL-Modell von Schulte-Körne ........................................................... 174

6.3.2.2.3. Bedeutung von Arbeitsgedächtnis bzw. phonologischer Bewusstheit bei der Verursachung

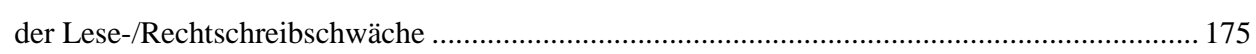




\subsection{DisKUSSION DES GESAMTBILDES NACH ASPEKTEN}

6.4.1. Zusammenhang zwischen den prädiktorischen Bereichen..... 177

6.4.2. Spezifikation des Verarbeitungsdefizits bei der Lese-/Rechtschreibschwäche

6.4.3. Das Kriterium der Verarbeitungspräzision 180

6.4.4. Bedeutung des Arbeitsgedächtnis im Vergleich zur phonologischen Bewusstheit........ 181

6.4.5. Methodische Überlegungen. 181

6.4.6. Diagnostische Nutzbarkeit des MMN-Paradigmas 182

7. ZUSAMMENFASSUNG

8. LITERATURVERZEICHNIS. 186

9. ANHANG

10. DANKSAGUNGEN 


\section{EINLEITUNG}

Die Not der Diagnostik einer Lese-/Rechtschreibschwäche besteht wie bei vielen Entwicklungsstörungen darin, dass im Kern der Diagnose eine nicht vorhandene bzw. sich nur langsam ausbildende Fähigkeit steht. Abwesenheit bzw. Langsamkeit in der Entwicklung ist im Allgemeinen nicht unbedingt auffällig, zumal das Defizit besonders bei den anfangs geringen Anforderungen mit anderen Mitteln - wie beispielsweise Auswendiglernen - kompensiert werden kann.

Bis die Leistungsschere gegenüber gesunden Kindern soweit aufgegangen ist, dass ein klinisch bedeutsamer Rückstand diagnostiziert werden kann, dauert es unter Umständen lange. Dieser Zeitraum beträgt im Falle der Lese-/Rechtschreibschwäche häufig zwei bis drei Jahre. Das hat Folgen.

Der Rückstand hat zu diesem Zeitpunkt ein Ausmaß erreicht, das auch bei normaler Begabung in der kritischen Fähigkeit kaum noch aufzuholen ist. Da der Lehrplan nun aber von einem angemessenen Maß schriftsprachlicher Kompetenzen ausgeht und auf dieser Grundlage weiter aufbaut, amplifizieren sich die Probleme nun. So hatte in der Mannheimer Längsschnittstudie (Esser, 1991; Esser \& Schmidt, 1994) bereits die Hälfte der Achtjährigen - bei durchschnittlicher Intelligenz - eine Klasse wiederholt und vier Fünftel hatten mangelhafte Leistungen in einem der Kernfächer. Obwohl 59\% der Gruppe mit Sprachentwicklungsstörungen (oder motorischen Entwicklungsstörungen) im Alter von 18 Jahren Realschul- oder Gymnasialniveau erreicht hatten, war dies bei nur 12\% der von einer Lese-/Rechtschreibstörung betroffenen Jugendlichen der Fall. Trotz für die Bewältigung des Alltags ausreichender Kompensationsbemühungen persistiert und beeinträchtigt die Lese-/Rechtschreibschwäche häufig über die Schulzeit hinaus während der gesamten Lebensspanne (Klicpera \& GasteigerKlicpera, 1993; Strehlow, Kluge, Müller \& Haffner, 1992).

Durch diese Belastung entstehen gravierende psychosoziale Probleme: $\mathrm{Zu}$ nennen sind hier Komorbiditäten und Sekundärstörungen wie Konzentrations- und Verhaltensprobleme (dissoziale Symptome, Hyperkinetisches Syndrom) und psychiatrische Störungen (McGee, Share, Moffitt, Williams \& Silva, 1988; Esser, 1991; Esser \& Schmidt, 1994). Die Quote psychisch auffälliger Kinder war in der Mannheimer Längsschnittstudie mit 61\% im Alter von acht und 13 Jahren und 41\% im Alter von 18 Jahren zwar nicht höher als die von Kindern mit anderen Entwicklungsstörungen, im Vergleich zur Normalpopulation (5 - 7\%; Remschmidt \& 
Walter, 1990) jedoch drastisch erhöht. Dementsprechend bedenklich fallen auch die sozialen Langzeitprognosen aus: Diese sprechen von 26\% Arbeitslosen einer lese-/rechtschreibschwachen Stichprobe im Alter von 25 Jahren gegenüber einem Arbeitslosenanteil von 4\% unter unauffälligen Kontrollpersonen und von 25\% in kriminelle Aktivitäten Verwickelte im Alter von 18 Jahren. Allerdings wird der hohe Anteil an Delinquenz / Dissozialität zum Teil auch einem Effekt niederer sozialer Schicht zugeschrieben (Maugham, Gray \& Rutter, 1985; Maugham, 1995; Naylor, Felton \& Wood, 1990).

Vor diesem Hintergrund wird der Nutzen deutlich, den eine vorschulische Diagnostik bringen könnte: Sie würde die Voraussetzungen für ein frühes und unter Umständen sehr wirksames Training schaffen. Mit dem Progamm „Hören, lauschen, lernen“ von Küspert und Schneider (1999; 2003) gelang es beispielsweise, die in der Schule verlangten schriftsprachlichen Fähigkeiten von Kindern, die im Kindergarten ehemals als Risikokinder eingeschätzt wurden, den Leistungen einer Kontrollgruppe anzugleichen (Küspert, 1998). Lundberg, Frost und Petersen hatten bereits zehn Jahre zuvor ein ähnliches Programm mit ähnlichem Erfolg entwickelt.

Im kognitionspsychologischen Forschungssektor herrscht folglich rege Tätigkeit, um schriftunabhängige Prädiktoren der Lese-/Rechtschreibschwäche zu identifizieren. Ein Ergebnis dieser Bemühungen ist das Bielefelder Screening (BISC) von Jansen, Mannhaupt, Marx und Skowronek (1998), das gefährdete Kinder u.a. anhand der sogenannten phonologischen Bewusstheit selegiert, einer sprachlichen Fähigkeit, die die Manipulation von Worten und Wortteilen erlaubt. Die phonologische Bewusstheit steht auch im Mittelpunkt der Trainings von Lundberg et al. (1988) und Küspert und Schneider (1999; 2003). Weitere potentielle Prädiktoren werden in Leistungen des phonologischen Arbeitsgedächtnisses sowie in der Aktivierung sprachlicher Codes des Langzeitgedächtnisses gesehen.

In jüngerer Zeit stellen sich an die Seite der kognitionspsychologisch orientierten Arbeitsgruppen auch neurophysiologischen Konzepten und Methoden verpflichtete Teams. Es ist von elektrophysiologischen Korrelaten der Lese-/Rechtschreibschwäche die Rede. Ein physiologischer Indikator könnte einen entscheidenden Vorteil gegenüber dem BISC aufweisen: Das BISC ist zum einen relativ zeitintensiv (ein bis zwei Stunden) und ist außerdem aufgrund des Eins-zu-eins-Settings zu aufwändig, um etwa in eine kinderärztliche Routineuntersuchung Eingang zu finden. So kommen Kinder im Augenblick nur in den Genuss des Screenings, wenn sie besonders aufmerksame Eltern haben, die bei Auffälligkeiten eine Fachstelle konsultieren, oder wenn sie Teilnehmer einer wissenschaftlichen Studie werden. 
$\mathrm{Da}$ das neurophysiologische Paradigma sich im gegenwärtigen Zustand erst in der Etablierungsphase befindet, muss das Ziel, aus diesem einen reliablen und erprobten Frühindikator der Lese-/Rechtschreibschwäche zu entwickeln, als Fernziel betrachtet werden.

Die ersten Schritte auf dem Weg müssten grundlagenwissenschaftliche Fragen klären. Deren primäre Aufgabe bestünde darin, die Prädizierbarkeit der Lese-/Rechtschreibschwäche durch die drei hauptsächlich genannten kognitionspsychologischen Konstrukte (phonologisches Arbeitsgedächtnis, phonologische Bewusstheit, Abruf aus dem Langzeitgedächtnis) sowie durch das neurophysiologische Paradigma an derselben Stichprobe abzusichern und miteinander $\mathrm{zu}$ vergleichen. Es interessierte dabei die Erklärungskraft der Prädiktion sowie die Tatsache, ob und welcher Art zwischen ihnen Beziehungen bestehen, dass heißt beispielsweise, ob die unterschiedlichen Prädiktoren als alternative Methoden zu sehen sind oder ob sie sogar geeignet wären, sich zu ergänzen. Weiterhin steht die Frage im Raum, ob vorhandene Beziehungen zwischen den Prädiktoren der Lese-/Rechtschreibschwäche nicht Ausdruck einer Kausalität der mit unterschiedlichen Methoden abgegriffenen Prozesse sein könnten.

Da das neurophysiologische Paradigma sich noch im Etablierungsprozess befindet, geht es auch darum, unterschiedliche Varianten $\mathrm{zu}$ entwickeln und zu testen. Aus solchen Versuchen ist anbei eine Aussage über ein potentielles neurophysiologisches Primärkorrelat der Lese/Rechtschreibschwäche zu erwarten.

Diesen grundlagenwissenschaftlichen Fragen stellt sich die vorliegende Studie. 


\section{THEORIE}

\subsection{DAS STÖRUNGSBILD "LESE-/RECHTSCHREIB- SCHWÄCHE“"}

Das Hauptkennzeichen der Lese-/Rechtschreibschwäche (LRS) besteht in einer besonderen Schwierigkeit im Erwerb von Lese- und Rechtschreibfähigkeiten, die nicht auf alternative Ursachen wie allgemeine Intelligenzminderung, unzureichende Beschulung oder emotionale bzw. neurologische Störungen zurückgeht.

2.1.1. HISTORISCHE ASPEKTE

Ein auffällig gestörter Schriftspracherwerb wurde 1885 erstmalig beschrieben. Jedoch brachte Berkhan (1885; 1886) die Störung dabei mit „Halbidiotie“ in Verbindung. Heute noch gültige ätiologische Kriterien zählte Morgan (1896) in seiner Hypothese zur ,angeborenen Wortblindheit" auf: Es handele sich um eine auf den Schriftspracherwerb beschränkte Schwäche einer ansonsten gesunden und normal intelligenten Person. Die Schwäche basiere auf einem genetisch bedingten visuellen Verarbeitungsdefizit, welches das Wortbildgedächtnis beeinträchtige. Den neben der „Lese-/Rechtschreibschwäche“ synonym gebräuchlichen Begriff der „Legasthenie“ führte Ranschburg (1916) ein. Dieser war es auch, der zum ersten Mal den Aspekt phonologischer Defizite in die Diskussion brachte, indem er auf Verwechslungsfehler nicht nur visuell, sondern auch phonologisch ähnlicher Buchstaben aufmerksam machte. Maria Linder (1951) schließlich griff Morgans Gedanken einer umschriebenen Störung auf und definierte die Legasthenie in Abgrenzung zu einer allgemeinen Lernschwäche als Teilleistungsstörung. Da dieses Konzept somit eine spezielle Schwäche im Schriftspracherwerb beinhaltet, die aus dem Rahmen der übrigen Leistungen eines normal begabten Kindes fällt, wurde es unter der Bezeichnung „Diskrepanzdefinition“ bekannt. Es bildet noch heute eine Säule der Diagnostik. 
2.1.2. BEGRIFFLICHKEIT

Neben den bereits erwähnten Begriffen „Lese-/Rechtschreibschwäche“ oder „Legasthenie“ wird diese Störung mitunter auch als „umschriebene Lese-/Rechtschreibschwäche“ bezeichnet, um die Erfüllung des in den Klassifikationsschemata geforderten Doppelkriteriums (siehe Punkt 2.1.3.) hervorzuheben. Dem sei in der vorliegenden Untersuchung mit der Bezeichnung „Lese-/Rechtschreibschwäche“ allein Genüge getan.

Im englischen Sprachgebrauch findet der Begriff "Dyslexie" Verwendung. Wie der Begriff „Legasthenie“ fokussiert er die Leseschwäche, was das Hauptaugenmerk von Forschung und Diagnostik auf das Lesen lenkt. In den 70'er Jahren wurde der „Legasthenie“-Begriff aufgrunddessen im deutschen Sprachraum massiv kritisiert und weitgehend durch „Lese-/Rechtschreibschwäche" ersetzt.

\subsubsection{KLASSIFIKATION UND PRÄVALENZ}

Die Lese-/Rechtschreibsstörung ist nach ICD 10 (1993) unter den „umschriebenen Entwicklungsstörungen schulischer Fertigkeiten“ als „Lese- und Schreibstörung (F 81.0)“ und im DSM IV (Saß, Wittchen \& Zandig (Hrsg.), 1998) als „Lesestörung (315.00)“ unter den „Lernstörungen" klassifiziert. Auch die Lesestörung schließt Rechtschreibschwierigkeiten ein. Die ICD 10 (Weltgesundheitsorganisation; Dilling, Mombour \& Schmidt (Hrsg.), 1993) führt weiterhin die „Isolierte Rechtschreibstörung (F81.1)“ an, bei der keine begleitende Lesestörung auftritt. Die Diagnostik einer Lese-/Rechtschreibsschwäche beruht auf der Kombination zweier Kriterien: Das Diskrepanzkriterium verlangt eine Differenz der Lese-/Rechtschreibleistung zur Intelligenz um mindestens zwei Standardabweichungen, wobei im klinischen Alltag besonders bei niedrigeren Intelligenzquotienten eine Diskrepanz von bereits einer Standardabweichung als klinisch relevant gewertet wird. Die Lese-/Rechtschreibleistung selbst muss weiterhin deutlich unter dem Durchschnitt, nämlich unter Prozentrang 10 liegen. Zwecks des Ausschlusses einer allgemeinen Intelligenzminderung wird ein Intelligenzquotient von mindestens 70, z.T. auch von mindestens 85 gefordert.

Schätzungen der Prävalenzrate reichen von 3 bis 10\% (Rutter, Tizard, Yule, Graham \& Whitmore, 1976). Dabei fanden die meisten Studien, dass das männliche Geschlecht zwei bis vier mal häufiger betroffen ist als das weibliche (Esser \& Schmidt, 1994). 


\subsubsection{KERNSYMPTOME}

Eine Lesestörung kann sich im frühen Entwicklungsstadium des Schriftspracherwerbs in Schwierigkeiten äußern, Buchstaben korrekt zu benennen. Später ist vor allem ein langsames Lesetempo kennzeichnend, da Wörter immer wieder Buchstabe für Buchstabe „erlesen“ werden müssen. Dementsprechend ist das Sinnverständnis häufig eingeschränkt. Lesefehler bestehen im Auslassen, Ersetzen, Verdrehungen oder Hinzufügen von Worten oder Wortteilen. $\mathrm{Zu}$ Beginn des Schreibenlernens treten Fehler der phonologischen Genauigkeit auf; später handelt es sich vor allem um Regelfehler. Die Schreibfehler können unterschiedlichster Art sein: Verdrehungen visuell identischer Buchstaben (b-d, n-u, p-q), Reihenfolgefehler (,dei" statt „die“), Buchstabenauslassungen (,ach“ statt „,auch“), Einfügen falscher Buchstaben („Artzt“ statt „Arzt"), Wahrnehmungsfehler (z.B. harter vs. weicher Konsonant) und Regelfehler. Schließlich kann dasselbe Wort in immer wieder unterschiedlicher Weise falsch geschrieben werden (Fehlerinkonstanz).

Da all diese Fehler jedoch auch bei Personen mit minderer Intelligenz auftreten, kann für die Lese-/Rechtschreibschwäche keine spezifische Fehlertypologie identifiziert werden.

\subsection{URSACHENBEREICHE DER LESE-/RECHTSCHREIB- $\underline{\text { SCHWÄCHE }}$}

Die Ursachen der Lese-/Rechtschreibschwäche sind nicht vollständig geklärt. Auf den verschiedensten Ebenen, wie z.B. molekularer, anatomischer, neurophysiologischer und kognitiver Ebene, werden Korrelate gefunden. Dies verdeutlicht die Komplexität des Störungsbildes. Bevor sich das Blickfeld der Studie auf die beiden Aspekte verengt, die im Fokus der vorliegenden Untersuchung stehen, sollen ausgewählte zentrale Ursachenhypothesen aus unterschiedlichen Forschungsansätzen kurz skizziert werden.

\subsubsection{GENETISCHE BEFUNDE}

In jedem Fall besteht eine genetische Disposition, vermutlich in Form eines autosomaldominanten Erbganges. Ist ein Kind betroffen, so beträgt das Störungsrisiko für Geschwister 52 bis $62 \%$ und 20 - $49 \%$ für Eltern (Schulte-Körne, Remschmidt \& Hebebrand, 1993; SchulteKörne, Deimel, Müller, Gutenbrunner \& Remschmidt, 1996; Froster, Schulte-Körne, 
Hebebrand \& Remschmidt, 1993; Grimm, Noethen, Schulte-Körne, 1998). Die Ergebnisse aus Zwillingsstudien zur Erblichkeit fallen allerdings inkonsistenter aus. Bezüglich eines Lesedefizits rangieren die Ergebnisse von $3-60 \%$, die Erblichkeit der Rechtschreibstörung wird auf 60 - 70 \% geschätzt (DeFries, Fulker \& LaBuda, 1987; DeFries, Stevenson, Gillis, \& Wadsworth, 1991; Stevenson, Graham, Fredman \& McLoughlin, 1987).

Die Vererbung ist vermutlich polygen. Kopplungs- und Assoziationsstudien wiesen auf eine Beteiligung der Chromosomen 1, 2, 3, 6, 15 und 18 hin. (zur Übersicht: Schulte-Körne, Grimm, Nöthen, Müller-Myhsok, Cichon, Vogt, Propping \& Remschmidt, 1998a; Grimm et al., 1998). Diese scheinen nicht die Lese-/Rechtschreibfähigkeit an sich, sondern dafür grundlegende Fähigkeiten wie „Wortlesen“ oder „phonologische Bewusstheit“ weiter zu geben.

\subsubsection{STRUKTURELLE UND NEUROLOGISCHE BEFUNDE}

Der strukturelle Hauptbefund im Gehirn lese-/rechtschreibschwacher Personen besteht im Fehlen oder in einer Inversion (Hier, LeMay, Rosenberger \& Perlo, 78; Haslam, Dalby \& Rademaker, 1981; Lubs, Smith, Kimberling, Pennington, Gross-Glenn, \& Duara, 1988) der bei der Mehrzahl der Bevölkerung auftretenden und für die Sprachverarbeitung wichtigen linkshemisphärisch dominanten Lateralisation der Plani temporali (Geschwind \& Levitsky, 1968). Weiterhin wird ein erhöhtes Vorkommen fokaler Dysplasien im Cortex berichtet (Galaburda, 1988; Galaburda \& Kemper, 1979; Galaburda, Sherman, Rosen, Aboitiz \& Geschwind, 1985; Kaufmann \& Galaburda, 1989), die zum Teil in Regionen der Sprachverarbeitung (perisylvanische Regionen) liegen. Beide Befunde werden von einem Überschuss an Neuronen sowie callosaler Konnektionen begleitet (Galaburda, Aboitiz, Rosen \& Sherman, 1986; Williams \& Rakic, 1988); die Symmetrie der Hemisphären schreiben Galaburda, LeMay, Kemper \& Geschwind (1978) nicht einem Minderwachstum des linken, sondern einer -wahrscheinlich durch inadäquates Neuronensterben bedingten- Vergrößerung des rechten Planums zu (Galaburda, 1991). Die Folge sei ein Verfehlen der optimalen Passung und damit einer optimalen Kommunikation neuronaler Strukturen.

John Locke (1997) erklärt in seiner „Theorie der neurolinguistischen Entwicklung“ die Lateralisationsanomalie mit einem verzögerten Einsatz des Worterwerbs, der dazu führt, dass einem linkshemisphärisch lokalisierten- potentiellen Sprachanalysemechanismus in einer dafür vorgesehenen kritischen Phase nicht ausreichend Material zur Verfügung steht. Ungenutzt verfalle somit die Analysefunktion der linken Seite und werde von der contralateralen Region über- 
nommen, welche allerdings für diese Aufgabe nicht adäquat ausgerüstet sei. Es komme zu einem bleibenden Kerndefizit in der Sprachanalyse bzw. in den phonologischen Fertigkeiten.

Mit der Methode des dichotischen Hörens und des Konzeptes der „right / left ear advantage (REA / LEA)“ wurde Bakker (1979) auf Besonderheiten in der lateralen zerebralen Organisation lese-/rechtschreibschwacher Kinder während des Leselernprozesses aufmerksam. Eine ear advantage besteht, wenn die auf ein bestimmtes Ohr besser als die auf das andere Ohr eingetroffene Information verarbeitet wird. Dies erlaubt den Rückschluss auf eine Dominanz der dem „bevorzugten“ Ohr contralateralen Sprachregion. Eine Verschiebung von LEAs zu REAs während des Leselernprozesses guter Leser veranlasste Bakker $(1979 ; 1986)$ zur Formulierung seines Balance-Modells: Das Lesen-Lernen gehe in einem zweiphasigen Prozess vonstatten: $\mathrm{Zu}$ Beginn dominiere die -rechtshemisphärisch kontrollierte- perzeptuelle Analyse der Schrift. Werden mit steigender Automatisierung des Leseprozesses zunehmend linguistische Aspekte einbezogen, verschiebt sich die Balance der zerebralen Kontrolle auf die linke Seite. Der Aufbau dieser zerebralen Dominanz sei bei lese-/rechtschreibschwachen Personen verzögert (Bakker, 1973; Satz, Rardin \& Ross, 1971), ein Befund, aufgrunddessen Bakker mittlerweile validierte Subtypen der Lese-/Rechtschreib-schwäche postulierte (Bakker, 1986; 1992).

Die fehlende Dominanz der linken Gehirnhemisphäre erachtete Orton (1937) als Ursache einer visuellen Gedächtnisstörung, die sich u.a. in der Verwechslung formidentischer Buchstäben äußere. Liberman, Shankweiler, Orlando, Harris \& Bell-Berti (1971) widerlegten diesen Ansatz jedoch, indem sie zeigten, dass Lesefehler vor allem auf phonetische, weniger auf visuelle Verwechslungen zurückgehen.

Veränderungen im Gyrus angularis (Duara, Kushch, Gross-Glenn, Barker, Jallad, Pascal, Loewenstein, Sheldon, Rabin, Lewin \& Lubs, 1991), welcher für die Übersetzung visueller in verbaler Information zuständig sei (Horwitz, Rumsey \& Donohue, 1998), sprechen für eine Hypothese, gemäß der die Hauptschwierigkeit lese-/rechtschreibschwacher Kinder im Verknüpfen visueller und akustischer / verbaler Information bestehe (vgl. Warnke, 1990; Warnke \& Wewetzer, 1997). Diesen Überlegungen zufolge wäre der intra- und interhemisphärische Informationsfluss gestört, ähnlich wie es auch bei den Überlegungen von Galaburda und Kollegen anklingt. 
In den magnozellulären Schichten des Nucleus geniculatus (Livingstone, Rosen, Dislane \& Galaburda, 1991) lese-/rechtschreibschwacher Personen finden sich Anomalien der Migration; diese Zellen selbst seien außerdem kleiner und variabler in der Größe (Galaburda \& Livingstone, 1993). Da im Nucleus geniculatus Neuronen aus der Retina mittels Punkt-zu-PunktVerknüpfungen v.a. mit dem Bereich der visuellen Hirnrinde verschaltet werden, der für visuelle Informationsverarbeitung mit hoher Geschwindigkeit und geringen Kontrasten verantwortlich gemacht wird, postulierten Galaburda \& Livingstone bei lese-/rechtschreibschwachen Personen eine geringere Sensibilität gegenüber schneller und kontrastarmer visueller Stimulation. Tatsächlich zeigten sich schon $50 \mathrm{~ms}$ nach Reizbeginn Abnormaltiäten im visuell evozierten Potenzial über den occipitalen Regionen. Auch andere Studien fanden eine Verzögerung früher Komponenten visuell evozierter Potenziale (Hennighausen, Remschmidt \& Warnke, 1994; Warnke \& Remschmidt, 1992).

In dieser Aufzählung wurden nur die wichtigsten Befunde aufgegriffen, um ein Bild der Hauptlinien biologischer Korrelate zu vermitteln. Wie stark jeder einzelne Befund zu den individuellen Schwierigkeiten eines lese-/rechtschreibschwachen Kindes beiträgt, ist schwer abschätzbar. Von einer Heterogenität innerhalb des Störungsbildes ist auszugehen. Doch wird deutlich, dass sich die Besonderheiten zerebraler Funktionen vor allem in zwei Bereichen der Informationsverarbeitung manifestieren müssen, nämlich der visuellen und der phonologischen Informationsverarbeitung. Vorstellbar sind auch Mängel der intrazerebralen Kommunikation. Da sich jedoch gezeigt hat, dass Beeinträchtigungen im visuell-räumlichen Bereich nur eine untergeordnete Rolle bei der Erklärung der Lernschwäche spielen (Warnke \& Remschmidt, 1992), der phonologischen Verarbeitung mittlerweile aber eine erstrangige Erklärungsrelevanz zugesprochen wird, konzentriert sich die aktuelle Forschung auf die phonologische Informationsverarbeitung.

\subsubsection{PHONOLOGISCHE INFORMATIONSVERARBEITUNG}

Die phonologische Informationsverarbeitung beinhaltet die Fähigkeit, lautsprachliche oder klangliche Strukturen der Sprache erkennen, manipulieren und nutzen zu können. Die funktionelle Einheit des phonologischen Moduls bildet das Phonem, welches als das kleinste klanglich unterscheidbare Segment von Sprache definiert ist.

Der Zusammenhang zwischen phonologischer Informationsverarbeitung und Lesefähigkeit ist gut belegt (z. B.: Adams, 1990; Brady \& Shankweiler, 1991; Goswami \& Bryant, 1990; Gough, 
Ehri \& Treiman, 1992; Sawyer \& Fox, 1991). Insbesondere auf der Basis von Langzeitstudien beruht die weitgehende Akzeptanz eines kausalen Einflusses der phonologischen Informationsverarbeitung auf den Schriftspracherwerb (Farmer \& Klein, 1995; Lundberg, Olofssson, Wall, 1980; Snowling, 2000; Treiman, 1991; Wagner \& Torgesen, 1987). Dies schließt einen bidirektionalen Zusammenhang (Wagner, Torgesen \& Rashotte, 1994) nicht aus. Ein schweres Defizit in der phonologischen Verarbeitung wird daher als bedeutender Risikofaktor für die Ausbildung einer Lese-/Rechtschreibschwäche gesehen (Bradley \& Bryant, 1978; Bruck, 1990; Mody, Studdert-Kennedy \& Brady, 1995; Rayner, Pollatsek \& Bilsky, 1995; Schulte-Körne, Deimel, Bartling \& Remschmidt, 1998b; Stanovich, 1991; Studdert-Kennedy \& Mody, 1995; Wagner, 1986).

Auch Studien, die sich der Einteilung leseschwacher Kinder in Subtypen widmen und damit ebenfalls Indizien bezüglich ätiologischer Fragestellungen bereitstellen, bestätigen konsistent das Vorkommen eines phonologischen Typs (Castles und Coltheart, 1993; Stanovich, Siegel \& Gottardo, 1997 sowie Manis, Seidenberg, Doi, McBride-Chang und Peterson, 1996). Mindestens 30\% der leseschwachen Kinder sind diesem Subtyp zuzuordnen. Shaywitz (1996) bringt ebenfalls in ihrem Modell des „Phonologie-Defizits“ schwache phonologische Verarbeitungsfähigkeiten mit der pathologischen Störung des Schriftspracherwerbs in Verbindung.

\subsubsection{NEUROPHYSIOLOGISCHE ANSÄTZE}

Mit neurophysiologischen Methoden werden Prozesse im sensorischen Gedächtnis sichtbar gemacht. Das auditive sensorische Gedächtnis -um dieses handelt es sich bei Lese/Rechtschreibschwäche-bezogener Forschung- bewerkstelligt die erste Speicherung der Information nach deren Wahrnehmung. Von dort aus werden die Gedächtnisspuren an andere zerebrale Funktionen weitergegeben.

Auf dem Gebiet der neurophysiologischen Forschung konnten mittlerweile einige wichtige Korrelate der Lese-/Rechtschreibschwäche identifiziert werden. Da es in der Natur der neurophysiologischen Methoden liegt, mit sehr distinkten Reizqualitäten zu experimentieren, sind die Korrelate in der Regel sehr spezifisch, was diese Herangehensweise wiederum besonders vielversprechend macht. Möglicherweise aufgrund dieser Spezifität sind die Befunde der unterschiedlichen Autoren zum aktuellen Zeitpunkt noch nicht unbedingt in Einklang zu bringen. Da auf diesem Feld jedoch rege Forschungstätigkeit herrscht, kann in nicht allzu ferner Zukunft auf Fortschritte in dieser Frage gehofft werden (- unter anderem durch die vorliegende Arbeit). 
Bei den am intensivsten diskutierten Linien Lese-/Rechtschreibschwäche-bezogener Befunde handelt es sich um die folgenden: Tallal (1980) begründete eine Theorie, gemäß welcher der Lese-/Rechtschreibschwäche eine Verarbeitungsschwäche rasch folgender auditiver Reize zugrundeliegt. Andere Autoren halten allein die Verarbeitung sprachlicher Stimuli für unterentwickelt (z.B.: Jaramillo et al., 1999; Mody, 1993; Mody, Studdert-Kennedy \& Brady, 1995; Schulte-Körne et al., 1998b; Studdert-Kennedy \& Mody, 1995). Es existiert weiterhin eine Vielzahl an Befunden, die sich im Sinne eines generellen auditiven Defizits interpretieren lassen, welches die gesamte auditive Informationsverarbeitung gleichermaßen beeinträchtigt (z.B.: Csépe \& Gyurkócza, 1995; Kujala, Karma, Čeponiene, Belitz, Turkkila, Tervaniemi \& Näätänen, 2001).

\subsection{KOGNITIONSPSYCHOLOGISCHE KONZEPTE UND METHODEN}

Wagner und Torgesen (und Rashotte) (Wagner \& Torgesen, 1987; Wagner, Torgesen \& Rashotte, 1994) unterteilten die phonologische Informationsverarbeitung in drei Komponenten, die phonologische Bewusstheit, den Abruf phonologischer Codes aus dem Langzeitgedächtnis und das phonetische Rekodieren im Arbeitsgedächtnis, und brachten alle drei Bereiche mit der Fähigkeit zu lesen in Verbindung.

\subsubsection{PHONOLOGISCHE BEWUSSTHEIT}

Die phonologische Bewusstheit beinhaltet die Fähigkeit, Phoneme (einzelne Phoneme sowie Phonemverbindungen) zu identifizieren, wiederzuerkennen und $\mathrm{zu}$ manipulieren. Demnach sind der phonologischen Bewusstheit die Lautanalyse und die Lautsynthese zuzuordnen. Das Erkennen klanglicher Gemeinsamkeiten und damit die Möglichkeit, bekannte Buchstabenmuster wieder einsetzen zu können, reduziert die Gedächtnisanforderung beim Lesen- und Schreibenlernen drastisch. Die Koartikulation jedoch macht es phonologisch gering begabten Kindern schwer, die Phoneme aus dem Wortkontext zu lösen, da sie die Worte als Einheiten wahrnehmen. Zudem verändert die Koartikulation die Phoneme um Nuancen; es entstehen unendlich viele „Allophone“. Phonologisch schwache Kinder haben infolgedessen Schwierig- 
keiten mit der sicheren Zuordnung von Allophon zu Phonem, und damit von Phonem zu Graphem.

Die phonologische Bewusstheit, die auf der Operation mit einzelnen Phonemen basiert, wird auch als ,phonologische Bewusstheit im engeren Sinne‘ bezeichnet; unter dem Begriff der ,phonologischen Bewusstheit im weiteren Sinne، wird auch die Fähigkeit zur Operation mit ganzen Silben oder Wortteilen verstanden.

Die phonologische Bewusstheit stand bislang im Fokus der Aufmerksamkeit zur Vorhersage des Schriftspracherwerbs. Ihre wesentliche Rolle dabei ist klar belegt (z. B.: Stanovich, 1988; Schneider, 1997; Schneider \& Näslund, 1993); Marx, 1997). Lundberg et al. (1980); zum Überblick: Goswami \& Bryant, 1990; Gough, Ehri \& Treiman, 1992).

$\mathrm{Zu}$ den einflussreichsten Arbeiten gehören die der Gruppe um Peter Bryant und Lynette Bradley. Ihre Untersuchungen bezogen sich v.a. auf die Reim- oder Lautkategorisierungsfähigkeit. Sie zeigten unter anderem, dass die phonologische Bewusstheit im Vorschulalter, trotz Kontrolle von Intelligenz und Gedächtnisspanne, auf vier Jahre später erhobene Fähigkeiten im Schriftsprachbereich einen bedeutenden Einfluss ausübte (Bradley \& Bryant, 1985). Die Formulierung einer Kausalität des Zusammenhangs beruhte auf dem Befund, dass die Beherrschung von Kinderreimen im Alter von drei Jahren die phonologische Bewusstheit vorhersagte, und diese wiederum in engem Zusammenhang mit späterem Schriftspracherwerb stand (Bryant, Bradley, MacLean \& Crossland, 1989; MacLean, Bryant \& Bradley, 1987).

Es ist allerdings möglich, dass erst der Schriftspracherwerb selbst die phonologische Bewusstheit ermöglicht, wie es Studien mit erwachsenen Analphabeten nahelegen (Morais, Cary, Alegria \& Bertelson, 1979; Read, Zhang, Nie, \& Ding, 1986). Da aber auch schon bei Kindergartenkindern eine gewisse Kenntnis der Schriftsprache nicht ausgeschlossen werden kann, sollten Trainingsstudien Antwort zur Art des Zusammenhangs zwischen phonologischer Bewusstheit und Erwerb der Schriftsprache geben. Lundberg, Frost \& Peterson (1988) stellten noch nach vier Schuljahren eine Überlegenheit im Lesen und Schreiben in phonologischer Bewusstheit trainierter Kinder versus einer untrainierten Kontrollgruppe fest. Das Training war zu einem Zeitpunkt erfolgt, als die Kinder noch über keine Kenntnisse des alphabetischen Prinzips verfügten. Andere Studien erbrachten ähnliche Befunde (Blaesser, 1994; Schneider, Visé, Reimers \& Blaesser, 1994). Die Autoren sahen damit eine Kausalitätsannahme für begründet.

Gemäß der Salzburger Längsschnittstudie (Landerl und Wimmer, 1994) wie auch der LOGIKStudie (Weinert \& Schneider, 1987; 1992) scheint die phonologische Bewusstheit v.a. für die Vorhersage der Lesefähigkeit bedeutsam zu sein. Dies mag ein Grund dafür sein, dass der 
phonologischen Bewusstheit im englischen Sprachraum, in dem v.a. die Leseschwäche im Vordergrund des Störungsbildes steht, eine größere Bedeutung als im deutschen Sprachraum beigemessen wird (vgl. Mayringer \& Wimmer, 1999; vgl. Hasselhorn, Tiffin-Richards, Banaschewski, Woerner \& Rothenberger, 2000(b)).

\subsection{2.} ABRUF PHONOLOGISCHER CODES AUS DEM LANGZEITGEDÄCHT$\underline{\mathrm{NIS}}$

Im Langzeitgedächtnis gespeichertes Wissen liegt wahrscheinlich in der Struktur von Netzwerken vor. Inhaltliche Kenntnisse entstammen der sogenannten semantischen Wissensbasis. Für eine hohe Leistungsfähigkeit des Langzeitspeichers ist nicht allein dessen Volumen oder Vernetztheit ausschlaggebend; ebenso wichtig ist eine schnelle Aktivierbarkeit der abgelegten Inhalte. An dieser Stelle liegt wahrscheinlich eine weitere Schwäche der Verarbeitungsprozesse lese-/rechtschreibschwacher Personen vor:

Bei lese-/rechtschreibschwachen Personen scheint speziell der Zugriff auf phonologische Codes erschwert (Wagner \& Torgesen, 1987; Wolf \& Obregon, 1992). Unter der Bezeichnung „phonologische Codes“ werden die Namen, die Aussprache und die Betonung jeweils von Buchstaben, Zahlen und Bezeichnungen verstanden. Es ist einleuchtend, dass die Effizienz dieses Prozesses für den Erfolg beim Dekodieren von Wörtern zentral ist (Wolf, 1991).

Eine Möglichkeit, die Fähigkeit des Zugriffs auf das Langzeitgedächtnis zu operationalisieren, besteht in der Benennungsgeschwindigkeit für Objekte, Buchstaben, Farben oder Zahlen.

\subsubsection{DAS ARBEITSGEDÄCHTNIS (NACH BADDELEY \& HITCH, 1974)}

Unter dem Begriff „Arbeitsgedächtnis“ wird allgemein eine kognitive Funktionseinheit verstanden, die an jeder bewussten Informationsverarbeitung beteiligt ist. Sie akquiriert Information aus der Umwelt sowie aus der Wissensbasis, hält diese temporal aufrecht, manipuliert sie gegebenenfalls und kann sie wieder der Wissensbasis zur langfristigen Speicherung zuleiten. Der Einsatz dieser Verarbeitungsprozesse wird vom Arbeitsgedächtnis selbst gesteuert.

Badeners Arbeitsgedächtnis-Modell (1986, 1990), welches von ihm und Hitch 1974 erstmalig formuliert wurde, wird aufgrund seiner Bewährung in vielerlei Forschungsbemühungen als 
mittlerweile bedeutende und differenzierte Modellvorstellung u.a. grundlegender Sprachverarbeitungsprozesse akzeptiert. Aufgrunddessen soll ihm im Folgenden besondere Aufmerksamkeit zuteil werden:

\subsubsection{DAS ARBEITSGEDÄCHTNISMODELL: ÜBERBLICK}

Das Modell geht von der Existenz mindestens dreier kooperierender Instanzen aus. Die „Zentrale Exekutive“ ist in ihrer Funktion als Kontrollinstanz über zwei modalitätsspezifische Hilfssysteme an deren Aktivität beteiligt. Mittels begrenzter Aufmerksamkeitsressourcen reguliert sie Kontrollprozesse der Informationsverarbeitung wie z.B. Aufnahme, Auswahl oder Transfer in oder aus der Wissensbasis (Baddeley, 1996a,b). Eines der beiden Subsysteme ist für die kurzfristige Speicherung visuell-räumlicher Information zuständig („visuell-räumlicher Sketchpad“; Logie, 1995). Auf diese Funktion wird im Folgenden nicht weiter eingegangen, da sie nicht in die Verarbeitung gehörter Sprache eingebunden ist. Das andere Subsystem, die „phonologische Schleife“ oder auch „phonologisches Arbeitsgedächtnis“ (wenn im weiteren Textverlauf von „Arbeitsgedächtnis“ gesprochen wird, ist stets von diesem „phonologischen Arbeitsgedächtnis“ die Rede), hält sprachliche Informationen kurzfristig fest. Das Arbeitsgedächtnismodell weist diesem Subsystem eine bedeutende Rolle bei der Sprachverarbeitung $\mathrm{zu}$.

\subsubsection{ARCHITEKTUR DER PHONOLOGISCHEN SCHLEIFE UND INTERAKTI- ONEN INNERHALB DES GEDÄCHTNISSES}

Das System "phonologische Schleife" besteht wiederum aus zwei Untereinheiten, nämlich einem passiven akustischen bzw. phonetischen Speicher als einer Strukturkomponente und einem subvokalen Rehearsalprozess als einer prozessualen Komponente. Dies ist in der folgenden Abbildung (Abb. 1) dargestellt.

Um die Funktionsweise der phonologischen Schleife im Gesamtkontext, d.h. auch deren Interaktion mit anderen Ebenen und Elementen kognitiver Sprachverarbeitung, betrachten zu können, sind in der Graphik auch die zentrale Exekutive, die semantische Wissensbasis (Langzeitgedächtnis) und sensorische Kurzzeitspeicher (Register) dargestellt. Die Abbildung 
entspricht einer vereinfachten Fassung eines Rahmenmodells der Informationsverarbeitung von Hasselhorn (1992; S.85).

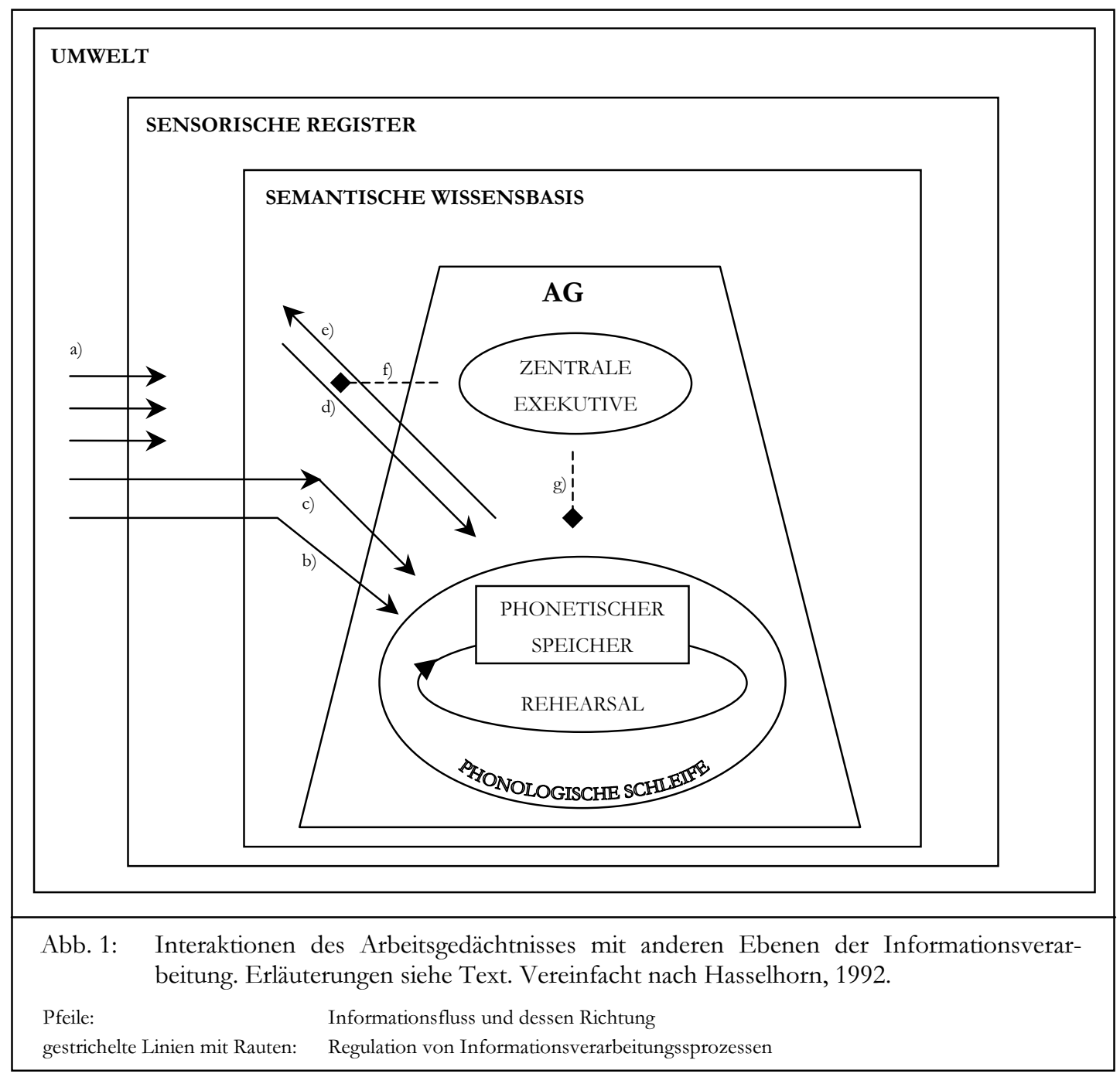

Perzeptionen aus der Umwelt gelangen in die sensorischen Register (a). Dabei erhalten sprachliche Wahrnehmungen automatisch Zugang zum phonetischen Speicher. Einige können in der Wissensbasis eine bereits vorhandene Repräsentation aktivieren (c), einige mögen noch unbekannt sein (b). Ebenso geht verbales Material aus dem Langzeitgedächtnis in den phonetischen Speicher ein (d). Die Spuren der Informationen liegen dort für ca. 1.5 bis 2 Sekunden vor (Baddeley, 1990; 1.8 sek: vgl. Hasselhorn, Grube \& Mähler, 2000c). In diesem Zeitraum können Ergebnisse aktueller Verarbeitungsvorgänge an kurz zurückliegende Verarbeitungsergebnisse angebunden werden, wodurch aus Phonemen Worte und anhand von Wortabschnitten Sinnzusammenhänge gebildet werden. Anders formuliert, verknüpft hier das Arbeitsgedächtnis Verarbeitungsresultate, bettet sie in die Dimension Zeit ein und schafft 
daraus das erst auf diese Art und Weise nutzbare Gesamtprodukt. Werden (neue) Spuren im phonetischen Speicher ausreichend lange aufrecht erhalten, so können stabile Repräsentationen in der Wissensbasis aufgebaut werden (e), die wiederum in den Speicher eingelesen werden können (d). Quantität und Qualität der shuttle-Prozesse zwischen Wissensbasis und phonologischem Arbeitsgedächtnis sowie die Aktivität innerhalb der phonologischen Schleife werden von der zentralen Exekutive beeinflusst (f).

Das subvokale Rehearsal besteht in einer Art inneren Sprechens, das die kurzlebigen Spuren im phonetischen Speicher gegebenenfalls so lange vor dem Verblassen bewahrt, bis eine langlebigere Repräsentation im Langzeitgedächtnis entstanden ist.

Eine weitere Aufgabe des Rehearsals besteht in der Übersetzung nicht akustisch dargebotenen, aber dennoch sprachlichen Materials wie Schrift, Symbole, Zahlen oder Bilder in einen phonetischen Code (,phonetisches Rekodieren“; Baddeley, 1982; 1986; Conrad, 1964). Erst anschließend geht die entsprechende Information in den phonetischen Speicher ein.

Anhand des Punktes d) dieses Schemas wird deutlich, dass eine der beiden vorgenannten Komponenten der phonologischen Informationsverarbeitung, nämlich der Abruf phonologischer Codes aus dem Langzeitgedächtnis, möglicherweise eine Leistung des Arbeitsgedächtnisses darstellt oder mit diesem zumindest in sehr engem Zusammenhang stehen könnte.

$\mathrm{Da}$ auch bei den Manipulationsvorgängen der phonologischen Bewusstheit Spuren aufrecht erhalten und Informationen neu verknüpft werden müssen, ist ebenfalls von einem deutlichen Zusammenhang zwischen Arbeitsgedächtnis und phonologischer Bewusstheit auszugehen. Sind diejenigen phonologischen Codes, deren Phoneme oder Silben es in einer bestimmten Aufgabe zu manipulieren gilt, als Referenzen oder Merkhilfen mühelos zu aktivieren, ist dies für die Bewältigung der Aufgabe sicherlich zuträglich. Ein enger Zusammenhang ist daher auch für die Konstrukte „Zugriff auf das Langzeitgedächtnis“ und „phonologischer Bewusstheit“ anzunehmen. 


\subsubsection{FUNKTIONSMERKMALE DER PHONOLOGISCHEN SCHLEIFE UND BEFUNDE DEFIZITÄRER VERARBEITUNG BEI LESE-/RECHTSCHREIB- SCHWACHEN PERSONEN}

\section{PHONETISCHER SPEICHER}

Die Speicherkapazität des phonetischen Speichers ist limitiert. Seine Begrenzung ergibt sich aus der Zeitspanne, innerhalb der aufgenommene Spuren aufrecht erhalten bleiben. Zur Messung der Kapazität des phonetischen Speichers wurde zu Beginn der 90'er Jahre von Gathercole, Willis, Emslie und Baddeley (1991) eine Aufgabenstellung entwickelt, in der Kunstwörter, d.h. unvertraute Klangkombinationen, nachgesprochen werden müssen (Gathercole, Willis, Baddeley und Emslie, 1994). Dasselbe Anforderungsprofil besitzt der auf Grete Mottier zurückgehende Test („Mottier-Test“), der für den deutschen Sprachraum bereits seit 1951 verfügbar ist. Da die Kunstwörter in kürzerer Zeit als die postulierte Behaltensdauer des phonetischen Speichers ausgesprochen werden können und die Probanden das Wort außerdem sofort nach dem Hören nachsagen, gehen Baddeley, Gathercole \& Papagno (1998) davon aus, dass es nicht zum Einsatz von Rehearsal kommt und somit allein die Fähigkeit zur Speicherung unbekannter Klangsequenzen abgefragt wird. Die Leistung beim Kunstwörter Nachsprechen korreliert mit Lese- und Schreibschwierigkeiten (Gathercole \& Baddeley, 1990).

Es wird vermutet, dass bei lese-/rechtschreibschwachen Personen v.a. der phonetische Speicher betroffen ist (Gathercole \& Baddeley, 1993; siehe auch Hasselhorn, Tiffin-Richards, Woerner, Banaschewski \& Rothenberger, 2000(b)).

Ein Einfluss lexikalischen Vorwissens bei wortähnlichen Kunstwörtern kann nicht abgestritten werden; es stellte sich jedoch heraus, daß dieser die Validität des Indikators nicht beeinträchtigt (Hasselhorn \& Seidler, 1999; Hasselhorn, Seidler-Brandler \& Körner, 2000(a)).

Hasselhorn und Kollegen experimentierten mit einer Variante des Kunstwörter Nachsprechens, in der die wiederzugebenden Worte verzerrt worden waren (Hasselhorn \& Körner, 1997). Beispielsweise reproduzierten Dysphasiker zwar lange Kunstworte schlechter als gesunde Kontrollprobanden. Beim Nachsprechen verzerrter Kunstworte waren die Muster der beiden Gruppen jedoch dieselben. Aus diesem Befund schlossen die Autoren, dass der phonetische Speicher nicht nur durch das Funktionsmerkmal Größe, sondern ebenfalls hinsichtlich seiner Verarbeitungspräzision charakterisierbar sei (Hasselhorn et al., 1995; Hasselhorn, 1999; vgl. Grube, Hasselhorn \& Weiß, 1998) und schlugen als empirischen Indikator das Nachsprechen verzerrter Kunstworte vor. 
Lese-/rechtschreibschwache Kinder schnitten sowohl beim Nachsprechen verzerrter Kunstwörter als auch im Mottier-Test im Vergleich zu Kindern einer Kontrollgruppe schlechter ab (Hasselhorn et al., 2000b).

\section{REHEARSAL}

Die Kapazitätsbegrenzung der prozessualen Komponente besteht in der Geschwindigkeit des Rehearsals. Je schneller das innere Wiederholen des zu merkenden Inhalts vonstatten geht, desto mehr Material kann im phonetischen Speicher aktiv gehalten werden (Baddeley, 1986, 1990). Auf diese Art und Weise kommt der sogenannte „Wortlängeneffekt“ zustande, der darin besteht, dass die Merkleistung mit steigender Wortlänge bzw. Silbenzahl sinkt (Baddeley, 1994).

Das subvokale Rehearsal ist mit höheren Sprachplanungsprozessen kongruent, die auch für die offene Artikulation genutzt werden (Baddeley \& Wilson, 1985; Gathercole \& Baddeley, 1993), so dass offene Artikulation die Rehearsalfunktion besetzt. Zwischen Sprechgeschwindigkeit und Reproduktionsleistung in Gedächtnisspannenaufgaben konnten klare Beziehungen gefunden werden: Je langsamer die maximale Sprechgeschwindigkeit eines Kindes ausfällt, desto geringer ist auch seine Behaltensleistung; erhöht sich die Sprechgeschwindigkeit (z.B. im Laufe des Älterwerdens), kann sich das Kind auch mehr Items merken (Hulme, 1984). Aus diesem Grunde wird die Sprechgeschwindigkeit zur Schätzung der Rehearsal-Geschwindigkeit herangezogen (Landauer, 1962).

Die Merkleistung schneller Leser erwies sich als der langsamer Leser überlegen (Baddeley, Thompson \& Buchanan, 1975).

Auch für das Rehearsal schlagen Hasselhorn, Grube \& Mähler (2000c) eine Charakterisierung anhand zweier Funktionsmerkmale vor: Neben der Geschwindigkeit wird auf den Automatisierungsgrad der Aktivierung hingewiesen. Im Rahmen der vorliegenden Forschungsarbeit wird das Rehearsal jedoch allein anhand seiner Geschwindigkeit beschrieben.

Die soeben geschilderte Aufgliederung des Arbeitsgedächtnisses ist durch die untenstehende Abbildung (Abb. 2) illustriert: 


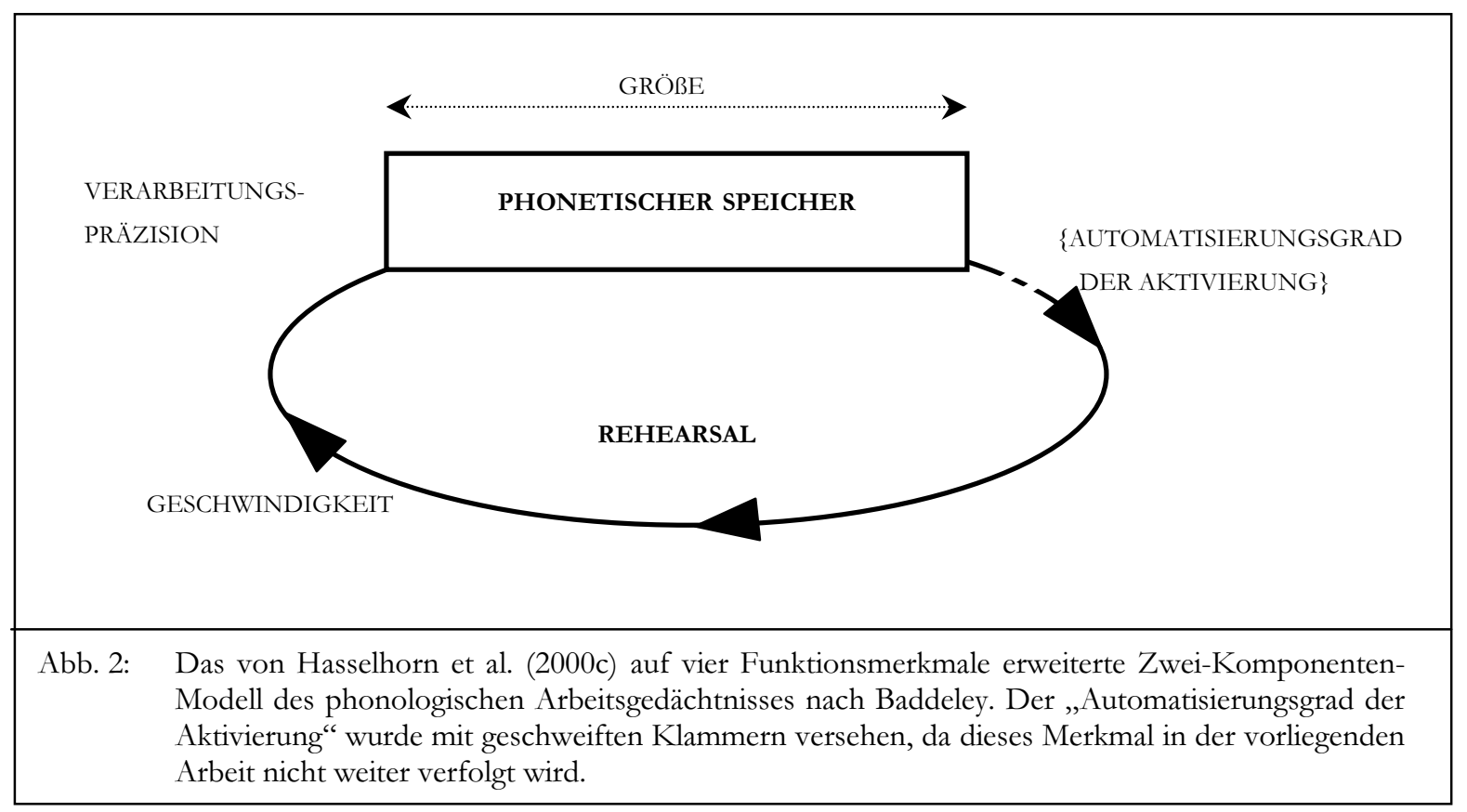

\section{PHONOLOGISCHE SCHLEIFE ALS GESAMTSYSTEM}

Dem Gesamtsystem der phonologischen Schleife weisen Gathercole \& Baddeley (1993) und Baddeley et al. (1998) -v.a. aufgrund ihrer Funktion, unbekannte Klangmuster zu speichern, während im Langzeitgedächtnis permanente Gedächtnisspuren konstruiert werden- eine Schlüsselrolle beim Sprachverständnis, beim Wortschatzerwerb und beim Lesenlernen zu.

Ihre funktionale Gesamtkapazität, welche auf der gemeinsamen Aktivität von Speicher und Rehearsal beruht, kann mithilfe der Gedächtnisspanne bestimmt werden (Gathercole \& Baddeley, 1993; Baddeley et al., 1998). Diese entspricht der Anzahl Items (Worte oder Zahlen), die eine Person aus einer Sequenz einmal gehörter Items korrekt und in richtiger Reihenfolge reproduzieren kann und gilt als verlässlicher Prädiktor für die Lese-, Rechtschreib- und Sprachfähigkeit. Lese-/rechtschreibschwache Kinder weisen sehr häufig eine verminderte Gedächtnisspanne auf (z.B.: Gathercole, Willis, Emslie \& Baddely, 1992; Gathercole, 1997; de Jong, 1998). 


\subsubsection{REPRÄSENTATIONSFORMAT INNERHALB DER PHONOLOGISCHEN SCHLEIFE}

Um Fragestellungen bezüglich des Repräsentationsformats der Inhalte der phonologischen Schleife nachzugehen, lässt sich der Effekt irrelevanter Sprache nutzen: Sprache gelangt automatisch ins phonologische Arbeitsgedächtnis. Für eine bestimmte Aufgabe (z.B. Gedächtnisspanne) irrelevante Anteile konkurrieren mit den relevanten Anteilen um die Kapazitäten der phonologischen Schleife. Die Leistung in der entsprechenden Aufgabe ist infolgedessen gemindert. Ebenso wie irrelevante Sprache sollten diejenigen akustischen Reize die Verarbeitung innerhalb der phonologischen Schleife beeinträchtigen, die zwar möglicherweise nicht sprachlich sind, aber dennoch vom phonologischen Arbeitsgedächtnis aufgenommen werden.

\section{KRITERIUM DER „SPRACHLICHKEIT“}

Baddeley (1986) hielt die phonologische Schleife für ein spezifisch an die Verarbeitung von Sprache angepasstes System. Da artikulierter Sprachschall, nicht aber Rauschen, die Gedächtnisspanne minderte, schlossen er und Salamé (1986) auf einen Filter, der nur sprachlichem und sprachähnlichem Material Zugang zur phonologischen Schleife gewähre.

Das wirft jedoch die Frage auf, anhand welcher Merkmale die „Sprachlichkeit“ des akustischen Materials erkannt wird. Das Vorhandensein oder die Abwesenheit dieser kritischen Merkmale bei sprachlichem sowie bei nichtsprachlichem Material würde infolgedessen über den Zutritt zur phonologischen Schleife entscheiden. Dass Rauschen die Merkleistung wenig beeinträchtigt, weist darauf hin, dass eine hohe spektrale Komplexität per se kein solches kritisches Merkmal sein kann. Sprache in Form einer repetitven Hintergrundsilbe zeigte ebenfalls keinen Effekt auf die Merkleistung (Jones, 1993). Wohl aber die tonale Variation der Hintergrundsilbe wie auch die Präsentation einer Serie vier variierender Töne störte die Gedächtnisspannenleistung (Jones \& Macken, 1993). Dies führte Jones und Macken (1993) zur Hypothese, dass für die Zulassung zur phonologischen Schleife nicht der Zustand des akustischen Materials selbst, sondern die Art seiner Zustandsveränderung bedeutsam sei (changing state hypothesis, Jones \& Macken, 1993). 


\section{ZEITKRITERIUM}

Klatte \& Hellbrück (1993) sahen in der spezifisch zeitlichen Struktur ein kritisches Merkmal für den Störeffekt akustischer Stimuli auf die Gedächtnisspannenleistung. Sie entnahmen diese Schlussfolgerung ihrem Befund, dass die Merkleistung durch schnelle Flötenmusik stärker beeinträchtigt wurde als durch Meditationsmusik. Jack (1990) erzielte mit SchreibmaschineTippen ebenfalls eine Minderung der Gedächtnisleistung und bestätigte somit den Eindruck von Klatte und Hellbrück. Durch diese Untersuchungen inspiriert, stellte Grube (1999) seiner Dissertation die Frage voran, inwieweit die Wiedergabe von Rhythmus von der phonologischen Schleife unterstützt würde. Zu diesem Zweck untersuchte er den Zusammenhang zwischen der Reproduktion eines Rhythmusses und Indikatorfähigkeiten für die Komponenten des phonologischen Arbeitsgedächtnisses. Sowohl mit der Gedächtnisspanne als auch mit der Artikulationsgeschwindigkeit fand Grube eine Korrelation der Rhythmuswiedergabe. Die Reproduktion unterlag außerdem einem (dem Wortlängeneffekt analogen) „RhythmuslängenEffekt", was auf eine Beteiligung des phonetischen Speichers schließen ließ. Von einer Beteiligung des phonologischen Arbeitsgedächtnisses an der kurzfristigen Speicherung zeitlicher Strukturen bzw. Rhythmen kann aufgrund dieser Befunde ausgegangen werden.

\subsubsection{RELEVANZ DES ARBEITSGEDÄCHTNISSES FÜR DEN SCHRIFT- SPRACHERWERB}

Im englischen Sprachraum wird dem Arbeitsgedächtnis -im Vergleich zur phonologischen Bewusstheit und dem Abruf aus dem Langzeitgedächtnis- für die Vorhersage von Lese/Rechtschreibfähigkeiten eine relativ geringe Bedeutung zugemessen (z.B. Wagner, Torgesen, Rashotte, Hecht, Berker, Burgess, Donahue \& Garon, 1997; Wolf \& Bowers, 1999). Die Schlussfolgerung, das phonologische Arbeitsgedächtnis spiele für den Schriftspracherwerb keine Rolle, halten Hasselhorn et al. (2000b) aus folgenden Gründen jedoch für voreilig: Die Übertragbarkeit von Konzepten, die für die Dyslexie des englischen Sprachraums entwickelt wurden, ist gegenüber der Lese-/Rechtschreibschwäche des deutschen Sprachraums möglicherweise eingeschränkt, da bei der Dyslexie das Lesedefizit, bei der Lese-/Rechtschreibschwäche das Schreibdefizit im Mittelpunkt steht. Möglicherweise stellt besonders das Schreiben Anforderungen an das phonologische Arbeitsgedächtnis.

Die Ergebnisse von Längsschnittstudien im deutschen Sprachraum deuten in diese Richtung (Klicpera \& Gasteiger-Klicpera, 1995; Näslund \& Schneider, 1996; Schneider \& Näslund, 1993; Weinert \& Schneider, 1987, 1992): Alle drei Komponenten der phonologischen Informations- 
verarbeitung konnten spätere Lese-/Rechtschreibleistungen vorhersagen. Zudem wirft der Befund, dass die Prädiktionswerte auch der Leseleistung durch den phonetischen Speicher im deutschen Sprachraum höher als im englischen Sprachraum ausfallen, die Vermutung auf, die durchschnittlich längere Wortlänge im Deutschen als im Englischen belaste das phonologische Arbeitsgedächtnis stärker.

Bei einer Subklassifikation innerhalb der phonologischen Dyslexie (Stanovich, 1991: phonological-core variable-differences vs. garden-variety; Castles \& Coltheart, 1993: phonologische vs. Oberflächen-Dyslexie), die sie durch Gruppierung nach Defiziten in einer, zwei oder allen drei Komponenten der phonologischen Informationsverarbeitung erhielten, kamen Morris, Stuebing, Fletcher, Shaywitz, Lyon, Shankweiler, Katz, Francis \& Shaywitz (1998) auf drei von fünf Subgruppen bzw. auf $85 \%$ der insgesamt zugeordneten Kinder mit Defiziten im phonologischen Arbeitsgedächtnis. Hasselhorn et al. (2000b) konnten weiterhin das gesamte Spektrum beobachteter Bereiche sprachlicher Minderleistungen (fünf Bereiche) von lese/rechtschreibschwachen Kindern durch Maße des phonetischen Speichers erklären. Nur ein Teil (zwei) der Bereiche identifizierter Minderleistungen ließen sich durch eine reduzierte phonologische Bewusstheit erklären.

Diese Befunde verdeutlichen die Relevanz des phonologischen Arbeitsgedächtnisses für das Erlernen der Schriftsprache im deutschen Sprachraum. 


\subsection{NEUROPHYSIOLOGISCHE KONZEPTE UND METHODEN}

Die erste Etappe wahrgenommener akustischer und sprachlicher Reize ist die Verarbeitung im Rahmen des auditiven sensorischen Gedächtnisses. Von diesem gelangen die Informationen ins Arbeitsgedächtnis, und können von dort aus wiederum für eine dauerhafte Speicherung ins Langzeitgedächtnis übertragen werden.

\subsubsection{DAS KONZEPT DES AUDITIVEN SENSORISCHEN GEDÄCHTNISSES}

Cowan $(1984 ; 1988 ; 1995)$ postulierte für das auditive sensorische Gedächtnis ein integratives Modell: Er stellt es sich als ersten Gedächtnisspeicher vor, der die physikalischen Eigenschaften des rezenten auditiven Inputs aufnimmt, kurzfristig behält und dadurch die Information der weiteren Verarbeitung zur Verfügung stellt. Dieser Speicher, den er „sensorisches Kurzzeitgedächtnis" nennt, bildet sowohl auditive als auch phonologische Gedächtnisspuren. Diese Vorstellung des sensorischen Kurzzeitgedächtnisses teilt Cowan mit anderen Autoren (Näätänen, 1992; Neisser, 1967). Die Eigenschaften der eintreffenden Stimuli aktivieren schließlich korrespondierende Repräsentationen im Langzeitgedächtnis und bringen auf diese Art und Weise deren Spuren ins Kurzzeitgedächtnis.

Die Erstellung einer solchen Gedächtnisspur geschieht gemäß Broadbent (1958; 1984) automatisch. Ihre Lebensdauer schätzt Broadbent auf bis zu 10 Sekunden. In der Gedächtnisspur sind alle Eigenschaften der gespeicherten Stimulus-Information in integrierter Form und die gesamte gebündelte Information der zurückliegenden 5 bis 10 Sekunden enthalten.

\subsection{2.} UNTERSUCHUNGSMETHODEN ZUM AUDITIVEN SENSORISCHEN GE$\underline{\text { DÄCHTNIS }}$

Die aktuelle Forschung zum auditorischen Kurzzeitgedächtnis bedient sich hauptsächlich diskriminativer Paradigmata. Stimuli müssen anhand spezifischer Merkmale differenziert oder identifiziert werden. Diese Aufgabenstellungen werden v.a. mittels zweier methodischer Hauptansätze bewerkstelligt. Auf behavioralem Wege muss die Erkennensleistung durch einen Knopfdruck demonstriert werden. Eine Möglichkeit besteht darin, bestimmte Tasten bestimmten Stimulustypen zuzuordnen, so dass der Proband eine gehörte Stimulussequenz anhand der Tasten nachvollziehen muss (,temporal order judgement“, „TOJ“). Unkomplexere Varianten 
bieten das „Same-Different-Paradigma“, bei dem der Tastendruck das Urteil über Gleichheit bzw. Unterschiedlichkeit zweier aufeinanderfolgender Sequenzen beinhaltet, oder der Tastendruck auf einen Zielstimulus inmitten distraktorischer Stimuli.

Zur Untersuchung früher Verarbeitungsprozesse gewinnt weiterhin das EEG (Elektroencephalogramm) -Paradigma immer mehr an Bedeutung: Während der Darbietung von Reizen bzw. während des Ablaufs kognitiver Operationen wird ein EEG abgeleitet. Die dadurch ausgelösten Potenziale, die sogenannten „Ereigniskorrelierten Potenziale (Event related potential; ERP)“, reflektieren systematische neurale Aktivität während der Ereignisse oder kognitiven Operationen und erlauben somit eine Verknüpfung der psychologischen mit der physiologischen Ebene.

Im Kontext der Lese-/Rechtschreibschwäche hat sich in besonderer Weise das ERP-Paradigma der Mismatch Negativity (MMN) etabliert und soll von daher im Folgenden ausführlich erläutert werden.

\subsubsection{DAS MisMATCH NEGATIVITY - PARADIGMA}

Man erzeugt diese Komponente, indem Probanden einem Fluss repetitiver auditiver Stimuli (Standards) ausgesetzt werden, welcher durch zufällig verteilte andersartige auditive Stimuli (Deviants) unterbrochen ist. Die negativ gepolte hirnelektrophysiologische Reaktion auf den Deviant fällt stärker als auf den häufig repetierten Reiz aus. Zwischen den beiden Antwortkurven besteht eine Differenz; dieser „Mismatch“ bezeichnet die erfolgte zerebrale Detektion der Andersartigkeit des Deviants vom Standardstimulus (Näätänen, 1995; Näätänen, Gaillard \& Mäntysalo, 1978). Die Mismatch Negativity wird von daher nicht direkt gemessen, sondern im Anschluss an die Messung aus den Potenzialen von Standard und Deviant mathematisch ermittelt. Dies soll gemeint sein, wenn im weiteren Textverlauf aus Gründen sprachlicher Vereinfachung von „Ableitung“ oder „Messung“ der Mismatch Negativity die Rede ist.

Das Setting ist passiv, da der MMN-Generationsprozess als aufmerksamkeitsunabhängig gilt (Alho, Woods, Algazi \& Näätänen, 1992). Für die Ableitung sind besonders frontozentrale 
Positionen des Skalps geeignet. Das Potenzial tritt in einen Zeitraum von ca. $100-300$ ms nach Stimulus-Onset auf (Näätänen \& Alho, 1995).

Die Mismatch Negativity wird im aktuellen Forschungskontext als ein physiologisches Korrelat der auditorischen Stimulus-abhängigen Repräsentationen im sensorischen Gedächtnis begriffen (Näätänen \& Alho, 1995). Dabei gehen Näätänen und Winkler (1999) davon aus, dass die MMN Resultat eines diskriminativen Prozesses ist, der aktuelle Gedächtnisspuren mit vorangegangenen vergleicht. Dieser Prozess dient möglicherweise dazu, die Aufmerksamkeit auf potentiell wichtige Veränderungen der Umwelt zu lenken (Öhmann, 1979; 1992). Dies schließen Näätänen und Winkler (1999) u.a. aus Experimenten, in denen Probanden während einer Aufgabe auf das Auftreten abweichender Stimuli in einer aufgabenirrelevanten Sequenz von Tonreizen mit einer Leistungsverschlechterung reagierten (Escera, Alho, Winkler \& Näätänen, 1998; Jääskeläinen, Alho, Escera, Winkler, Sillanaukee \& Näätänen, 1996; Schröger, 1996).

Die im Folgenden darzustellenden Funktionsmerkmale charakterisieren die Mismatch Negativity. Es sind v.a. diese Merkmale, die die Attribution der MMN als Ausdruck von Repräsentationen innerhalb des auditiven sensorischen Gedächtnisses bedingen.

Die MMN integriert die Codierung verschiedener Merkmale zu einer funktionellen Einheit

Die Teilspuren, die der MMN zugrundeliegen, reflektieren die Merkmale der sensorischen Information. Je nachdem, durch welches Merkmal der Kontrast zwischen Standard und Deviant gebildet wird, variiert der Lokus der MMN-Generation im auditorischen Cortex (Giard, Lavikainen, Reinikainen, Bertrand, Pernier \& Nätänen, 1995; Levänen, Ahonen, Hari, McEvoy \& Sams, 1996; Paavilainen, Alho, Reinikainen, Sams \& Näätänen, 1991). Die neuronale Codierung der Eigenschaften ist demzufolge anatomisch verstreut. Nichtsdestotrotz bilden die Neuronenpopulationen eine funktionelle Einheit, wie es beispielsweise ein Experiment von Gomes, Bernstein, Ritter, Vaughan und Miller (1997) verdeutlicht: Als Standard wurden drei Stimulustypen präsentiert. Der Deviant bildete sich aus der Kombination der Eigenschaften zweier Standards und löste eine MMN aus. Würde der Detektionsprozess 
auf die isolierten physikalischen Eigenschaften des auditorischen Inputs reagieren, hätte keine MMN entstehen dürfen.

Die den Kontrast konstituierenden Merkmale beeinflussen das Erscheinungsbild der Kurve (Sandridge \& Boothroyd, 1996).

Als Mechanismus der Repräsentationsbildung schlagen Näätänen und Winkler (1999) ein gleitendes Zeitfenster von ca. $200 \mathrm{~ms}$ Ausdehnung vor, innerhalb dessen die Stimuluseigenschaften integriert werden. Die Integration erfolgt sowohl parallel als auch sequentiell.

Die MMN bildet sämtliche Merkmale eines Ereignisses ab

In den der MMN zugrundeliegenden Gedächtnisspuren werden sämtliche, das heißt, sowohl statische als auch dynamische Stimulusmerkmale der auditorischen Information erfasst. MMNs konnten durch Unterschiede in Frequenz (Näätänen et al., 1978), Intensität (Lounasmaa, Hari, Joutsiniemi \& Hämäläinen, 1989; Näätänen, Paavilainen, Alho, Reinikainen \& Sams, 1987) sowie durch die räumliche Veränderung der Tonquelle (Paavilainen, Karlsson, Reinikainen \& Näätänen, 1989; Schröger \& Wolff, 1996; Winkler, Tervaniemi, Schröger, Wolff \& Näätänen, 1998) ausgelöst werden. Ebenso erzeugen phonetische Unterschiede eine MMN (Kraus, McGee, Sharma, Carrell \& Nicol, 1992, Sandridge \& Boothroyd, 1996). Zeitgebundene Merkmale wie das Interstimulusintervall (Ford \& Hillyard, 1981; Näätänen, Jiang, Lavikainen, Reinikainen \& Paavilainen, 1993a; Nordby, Roth \& Pfefferbaum, 1988), rise time (Lyytinen, Blomberg \& Näätänen, 1992), Stimulusdauer (Kaukoranta, Sams, Hari, Hämäläinen \& Näätänen, 1989; Näätänen, Paavilainen \& Reinikainen, 1989), die Reihenfolge innerhalb einer Stimulussequenz (Winkler \& Schröger, 1995) sowie der Rhythmus der Abfolge (Imada, Fukuda, Kawakatsu, Masjiko, Odaka, Hayashi, Aihara \& Kotami, 1993) werden weiterhin in den der MMN zugrundeliegenden Spuren codiert.

Diese Befunde verdeutlichen zum einen, dass jeglicher wahrnehmbare auditorische Kontrast in der Lage ist, eine MMN auszulösen. Weiterhin wird deutlich, dass der Bildung der MMN nicht allein die „photographische“ Speicherung momentaner Information, sondern vielmehr die vollständige Erfassung des auditorischen Ereignisses inklusive seiner zeitlichen Dynamik zugrundeliegt. 
Die MMN steht in reziproker Beziehung zu Repräsentationen im Langzeitgedächtnis

Die sensorische Information der Gedächtnisspuren, die der MMN zugrundeliegen, initiiert wahrscheinlich die Erstellung dauerhafterer Repräsentationen. So zeigten Cowan und Kollegen (Cowan, Winkler, Teder \& Näätänen, 1993), dass für die Wiederauffrischung der verblassten Spur eines Standardstimulus (und damit zur Möglichkeit einer MMN-Auslösung) eine einmalige erneute Präsentation ausreichte. Näätänen, Schröger, Karakas, Tervaniemi \& Paavilainen (1993b) boten Probanden Muster komplexer Töne. Die Frequenz eines Tones in der Abfolge war beim Deviant verändert. Versuchspersonen, die den Unterschied nicht bewusst erkennen konnten, zeigten auch keine MMN. Lernten sie aber die Diskrimination im Laufe der Untersuchung, konnte auch die Ausbildung einer MMN beobachtet werden. Selbiges konnten Winkler, Kujala, Tiitinen, Sivonen, Alku, Lehtokoski, Czigler, Csépe, Ilmoniemi. \& Näätänen (1999) für das Erkennen phonetisch relevanter Unterschiede beim Erlernen einer Fremdsprache demonstrieren. Befunde dieser Art deuten auf die Etablierung einer dauerhafteren Gedächtnisspur hin, die im Weiteren die Grundlage des Diskriminationsprozesses bildet.

Cheour, Čeponiene, Lehtokoski, Luuk, Allik, Alho und Näätänen (1998) belegten in besonders eindrücklicher Weise die Bildung von Gedächtnisspuren im Langzeitgedächtnis, indem sie deren Rückwirkung aus der Wissensbasis auf die Diskriminationsleistung zeigten. Sechs Monate alten Finnen und Esten wurden die Vokale /o/, /ö/ und das intermediäre /õ/ dargeboten. Die Kinder beider Nationen diskriminierten die Stimuli anhand ihrer physikalischen Kriterien. Im Alter von einem Jahr jedoch hatte bei den Finnen die Diskrimination des in ihrer Sprache relevanten /o/ - /ö/ - Kontrastes deutlich zugenommen, die des nur im Estnischen relevanten /o/ - /õ/ - Kontrastes war dagegen etwas gesunken. Offenbar hatten die Spuren der bedeutsamen Phoneme stabile Repräsentationen im Langzeitgedächtnis erzeugt, welche als kategoriale Muster die Diskrimination der muttersprachlichen Phoneme fördern. Für sprachunrelevante Kontraste war die Bildung der Langzeitrepräsentationen ausgeblieben.

Diese Befunde zeigen, dass es sich bei der MMN um einen Ausdruck eines zwar frühen, aber dennoch in aktiver Wechselwirkung mit der Wissensbasis im Langzeitgedächtnis stehenden Verarbeitungsprozess auditorischer Information handelt.

Die beiden folgenden Charakteristika der MMN gehen aus dem soeben Erläuterten hervor. 
Die $M M N$ korreliert mit der Reizdiskrimination auf behavioraler Ebene

Der oben erwähnte Versuch von Näätänen et al. (1993b), in dem das Erlernen der Diskrimination durch die Entstehung einer MMN begleitet wurde, wie auch Winklers Demonstration, dass die Unterscheidung fremdsprachiger Phonemkontraste mit der Ausbildung von Mismatch Negativities in Zusammenhang stand (Winkler et al., 1999), weisen außerdem auf die enge Korrelation zwischen dem Indikator der physiologischen Diskrimination für einen bestimmten Kontrast und der behavioralen Diskriminationsleistung dieses Kontrastes hin (siehe auch: Aaltonen, Toumainen, Laine \& Niemi, 1993; Kraus, McGee, Carrell \& Sharma, 1995; Lang, Nyrke, Ek, Aaltonen, Raimo \& Näätänen, 1990).

MMN-Amplitude und Latenz korrelieren mit der Größe des Unterschieds der sensorischen Information

Die Größe des Unterschiedes der perzeptuellen Information findet in der MMN Ausdruck. Tiitinen, May, Reinikainen und Näätänen (1994) stießen auf eine proportionale Beziehung, als sie die Amplitude der MMN mit dem Frequenz-Unterschied zwischen Deviant und Standard verglichen. Ebenso unterlag die Latenz einem systematischen Zusammenhang. 


\subsubsection{BEFUNDE ZUM AUDITIVEN SENSORISCHEN GEDÄCHTNIS IM}

\section{KONTEXT VON LESE-/RECHTSCHREIBSCHWÄCHE}

In der Forschung zum auditiven sensorischen Gedächtnis werden drei Haupthypothesen bezüglich der Defizite bei lese-/rechtschreibschwachen Personen vertreten. Es handelt sich hierbei um ein Defizit der Verarbeitung zeitlicher Information bei raschen Stimulusfolgen, ein generelles auditives Verarbeitungsdefizit und schließlich um ein spezifisch die Verarbeitung sprachlicher Reize betreffendes Defizit. Die prinzipielle Wirkweise eines solchen Defizits wäre in allen genannten Vorstellungen ähnlich: Die Verarbeitungsdysfunktion behindere die Bildung phonologischer Kurzzeitrepräsentationen; dies wiederum beeinträchtige die Erstellung der Langzeitgedächtnisspuren. Ohne diese als kategorielle Vorlagen ist jedoch das Erkennen sprachlicher Elemente und damit der sichere Umgang mit Sprache erschwert (Fowler, 1991; Swan \& Goswami, 1997).

\subsubsection{DEFIZIT DER RASCH TEMPORALEN VERARBEITUNG}

Eine bekannte Theorie (Tallal, 1980; Tallal, Miller, Jenkins \& Merzenich, 1997; Tallal, Miller \& Fitch, 1993; Reed, 1989) versucht, die Schwierigkeiten lese-/rechtschreibschwacher Personen in der Sprachverarbeitung auf Schwierigkeiten in der zeitlichen Verarbeitung zurückzuführen. Tallal und ihre Kollegen experimentierten im „temporal order judgement“ und im „same / different" - Paradigma mit der Länge der Einzeltöne innerhalb der dargebotenen Tonsequenzen und mit der Dauer des Interstimulusintervalls. Lese-/rechtschreibschwache Kinder zeigten bei kurzen Interstimulusintervallen (unter $428 \mathrm{~ms}$ ) bzw. bei kurzen Tonlängen (75 ms statt $250 \mathrm{~ms}$ ) deutliche Leistungseinbußen. Daraus schloss Tallal auf ein grundlegendes Verarbeitungsdefizit zeitlicher Information, ein sogenanntes „temporal processing deficit“. Besonders StuddertKennedy und Mody (1995) präzisierten die Definition des Defizits, indem sie darauf hinwiesen, dass die Dysfunktion nur die temporale Verarbeitung schneller Stimulusfolgen betreffe. Unter der Bezeichnung „rasch temporales Verarbeitungsdefizit“ soll im weiteren Textverlauf ebendies verstanden werden. Die an anderer Stelle erwähnte Studie von Čeponiene et al. (1999) kann in diesem Sinne verstanden werden, da die Einschränkung der Diskriminationsleistung der schlecht Kunstwort nachsprechenden Gruppe vor allem bei kurzen Interstimulusintervallen von $350 \mathrm{~ms}$, nicht bei Intervallen von $2000 \mathrm{~ms}$, auftrat. 
Es muss einschränkend hinzugefügt werden, dass Tallal und ihre Kollegen Defizite der rasch temporalen Verarbeitung nur bei solchen Kindern gefunden hatten, deren Leseschwäche von Schwierigkeiten in der verbalen Sprachproduktion begleitet wurde. Nichtsdestotrotz erfuhr diese Hypothese in der Forschungslandschaft -verdientermaßen-ausgeprägte Beachtung, da sie, wie eine Studie von Shannon, Zeng, Kamath, Wygonski und Ekelid (1995) zeigt, einen kritischen Punkt der Sprachverarbeitung berührt: Hier gelang Probanden die Identifikation von Klängen, deren spektrale Information beinahe völlig reduziert war, sofern ihre temporale Information jedoch erhalten blieb.

Schulte-Körne, Deimel, Bartling und Remschmidt (1999a) überprüften die Hypothese des raschen temporalen Verarbeitungsdefizits anhand von Sequenzen unterschiedlicher Töne im MMN-Paradigma. Die Ergebnisse fielen erwartungsgemäß aus. Veränderungen der zeitlichen Abfolge der Töne riefen bei lese-/rechtschreibschwachen Probanden kleinere Mismatch Negativities als bei gesunden Kontrollpersonen hervor. Kujala, Myllyviita, Tervaniemi, Alho, Kallio und Näätänen (2000) arbeiteten mit einem rhythmischen Paradigma. Ein Gruppenunterschied der MMN wurde durch Vorversetzen des dritten Tons (von vieren) bewirkt. Dieselbe Manipulation erzeugte jedoch keinen Gruppenunterschied, wenn nur die veränderungstragenden Töne, das heißt, der zweite und der dritte Ton dargeboten wurden. Die Autoren schlossen infolgedessen auf einen Maskierungseffekt in der Vier-Ton-Bedingung. Sie brachten damit das temporale Verarbeitungsdefizit lese-/rechtschreibschwacher Personen in Verbindung mit einer erhöhten Vulnerabilität gegenüber Maskierungseffekten. Diese Vulnerabilität wiederum, so die Vermutung, könnte auf ein verlängertes Integrationsfenster bei der Erstellung der Repräsentationen im auditiven sensorischen Gedächtnis zurückgehen.

Besondere Probleme müsste gemäß Tallal et al. (1997) die Diskrimination derjenigen Phoneme stellen, die durch schnelle Veränderung der Frequenzen (Formanten), sogenannte Transitionen, entstehen. Es handelt sich hierbei um die Stop-Konsonanten (Verschlusslaute). Diese wären die Laute /k/, /g/, /t/, /d/, /p/ und /b/. Befunde wie beispielsweise von Reed (1989), gemäß welcher sich dyslektische Kinder bei Aufgaben zur raschen temporalen Verarbeitung gegenüber Kontrollkindern im Nachteil befanden, wenn die Stimuli aus Silben mit einem StopKonsonanten bestanden, nicht jedoch, wenn Vokale die Stimuli konstituierten, unterstützen die Position Tallals. Auch Mody et al. (1995) oder Schulte-Körne, Deimel, Bartling und Remschmidt (1998c, 1999b) konnten eine besondere Beeinträchtigung der Diskrimination von StopKonsonanten bei lese-/rechtschreibschwachen Personen nachweisen (siehe unten). 
Zeitliche Modifikationen innerhalb der Stop-Konsonanten untersuchten die beiden folgenden Gruppen. Sie variierten die Stimmhaftigkeit bzw. die wahrgenommene Dauer von Konsonanten, was durch eine Veränderung der Konsonantendauer bis zum Einsatz des folgenden Vokals (,voice onset time") vonstatten geht. Harte oder doppelte Konsonanten weisen dabei die längere voice onset time auf.

Chiappe, Chiappe und Siegel (2001) schufen zehnstufige Kontinua innerhalb zweier Silbenpaare. Jedes Paar bestand aus einem in der Landessprache sinnvollen und einem Kunstwort, wobei die beiden sich allein in der Stimmhaftigkeit des Startkonsonanten unterschieden (/bis/ versus /pis/; /bif/ versus /pif/). Bei der Kontrollgruppe trat erwartungsgemäß bei den mittleren Stufen im Kontinuum ein lexikalischer Effekt, d.h. eine überzufällig große Tendenz zu Entscheidungen für die sinnvolle Silbe, auf. Die Probanden verließen sich folglich im Falle akustischer Uneindeutigkeit vermehrt auf ihr lexikalisches Wissen. Bei lese-/rechtschreibschwachen Personen war dieser lexikalische Effekt über alle Kontinuumsstufen hinweg zu beobachten, was auf grundlegende perzeptuelle Identifikationsunsicherheiten für die Konsonanten /b/ bzw. /p/ schließen lässt.

Ähnliche Ergebnisse erbrachte die Gegenüberstellung von /ata/ und /atta/ im MMNParadigma von Leppänen und Kollegen (2002). Die Untersuchung wurde an ca. sechs Monate alten Säuglingen durchgeführt, die aufgrund ihrer familiären Herkunft mit dem Risiko einer zukünftigen Lese-/Rechtschreibschwäche belastet waren, sowie an einer altersparallelisierten Kontrollgruppe. Der MMN des Kontrastes „Standard /atta/ - Deviant /ata/“ fehlte bei den Risikokindern ein zweiter Peak; die Bedingung „Standard /ata/ - Deviant /atta/“ erzeugte bei Risikokindern (linkshemisphärisch) kleinere Differenzpotenziale.

Die Hypothese eines Defizits der raschen temporalen Verarbeitung inklusive aller konformen Befunde verlöre an Bedeutung, stünde sie mit phonologischen Fähigkeiten oder der Lese- oder Schreibschwäche nicht in Zusammenhang, sondern wäre als eine Art konsequenzlose Begleiterscheinung der Lese-/Rechtschreibschwäche zu verstehen. In diesem Punkt herrscht jedoch Uneinigkeit. Tallal und ihre Kollegen fanden eine erstaunlich hohe Korrelation von 0.81 ( $p<$ 0.001) zwischen dem Lesen von Kunstworten und nonverbalen Aufgaben zur temporalen Verarbeitung, die allerdings in dieser Höhe nicht wieder repliziert werden konnte. Andere Autoren belegten einen Zusammenhang zwischen der raschen temporalen Verarbeitung und Aufgaben zur phonologischen Bewusstheit (Booth, Perfetti, MacWhinney \& Hunt, 2000; Talcott, Witton, McClean, Hansen, Rees, Green \& Stein, 1999). Dem stehen auch Befunde entgegen, gemäß derer beispielsweise der Einfluss der raschen temporalen Verarbeitung auf 
phonologische Fähigkeiten bzw. auf das Lesen / Rechtschreiben gar nicht besteht (Watson \& Miller, 1993) oder nicht auf eine gemeinsame genetische Varianz zurückgeht (Bishop, Carlyon, Deeks \& Bishop, 1999). Auch Marshall, Snowling \& Bailey (2001) stießen auf deutliche Zusammenhänge zwischen der phonologischen Bewusstheit auf Phonemebene und der Lesefähigkeit sowie zwischen Aufgaben zur raschen temporalen Verarbeitung und der phonologischen Bewusstheit auf Silbenebene, nicht jedoch auf einen (direkten oder indirekten) Zusammenhang zwischen der raschen temporalen Verarbeitung und der Leseleistung.

Die These des raschen temporalen Verarbeitungsdefizits ist demzufolge als eine plausible und weithin anerkannte, aber dennoch nicht unwidersprochene Hypothese zu beurteilen.

\subsubsection{GENERELLES AUDITORISCHES VERARBEITUNGSDEFIZIT}

Dysphasiker produzierten in Untersuchungen von Hasselhorn und Kollegen (Hasselhorn, Hille, Süß, Werner \& Grube, 1995; Hasselhorn, 1999) einen erstaunlichen Befund: Im KunstwortNachsprechen nach Hasselhorn (Hasselhorn \& Körner, 1997), eine Aufgabe, deren schwierigkeitserhöhendes Prinzip zum einen in der Steigerung der Silbenzahl, zum anderen in der Verzerrung der dargebotenen Worte besteht, zeigten die dysphasischen Kinder im Gegensatz zu Kontrollkindern keine zusätzliche Verschlechterung der Wiedergabe langsilbiger Worte durch die Verrauschung. Eine mögliche Erklärung dieses Phänomens würde ein generelles auditorisches Verarbeitungsdefizit bieten, welches (noch) nicht wie das rasche temporale Defizit auf einen konkreten Funktionsbereich lokalisierbar ist, sondern sich wie ein Schleier über die Perzeption aller akustischen Modalitäten legt und damit zu unschärferen Langzeitrepräsentationen führt. Eine solche generelle auditorische Verarbeitungsschwäche wäre auch für die Lese-/Rechtschreibschwäche vorstellbar.

Ein generelles auditorisches Defizit müsste der Annahme eines temporalen Verarbeitungsdefizits nicht widersprechen, sondern könnte es einschließen oder nebenher bestehen.

Eine Verarbeitungsschwäche bei der Erkennung von Frequenzunterschieden konnten Baldeweg, Richardson, Watkins, Foale und Gruzelier (1999) nachweisen. Auf der Basis der Annahme, dass im Sprachverarbeitungssystem lese-/rechtschreibschwacher Personen möglicherweise die Diskrimination unterschiedlicher Modalitäten eingeschränkt ist, entwickelten Kujala und Kollegen (2001) ein Trainingsprogramm zur Verbesserung der Lesefähigkeit. Dieses Programm verzichtete auf lexikalische Elemente und beschränkte sich auf die Darbietung von 
Sequenzen von Tönen unterschiedlicher Frequenz, Dauer und Intensität, welche in kodierter Form auf einem Bildschirm visualisiert worden waren. Des Probanden Lernaufgabe bestand im aktiven „Mitlesen“ der Tonfolge. Bereits nach 14 zehnminütigen Durchgängen hatte sich bei den siebenjährigen lese-/rechtschreibschwachen Probanden nicht nur die MMN-Amplitude zu Kontrasten der trainierten Stimuli vergrößert, sondern auch die Lesefähigkeit verbessert. Der Erfolg dieses Programms unterstreicht weiterhin eindrücklich die Bedeutung der nonverbalen, generell auditiven Sprachverarbeitung für den Schriftspracherwerb.

Die Hypothese eines Defizits in der generellen auditiven Sprachverarbeitung muss von daher ebenfalls als bedeutend angesehen werden.

\subsubsection{SPRACHSPEZIFISCHES VERARBEITUNGSDEFIZIT}

Jaramillo und Kollegen (1999) stießen in ihrer Untersuchung zur Erkennung von Frequenzunterschieden auf Indizien für eine sprachspezifische Verarbeitung. Erzeugten sie den Deviant durch Verlängerung eines Sinuston-Standards, so entstand eine Mismatch Negativity, die größer als die Mismatch Negativity auf einen durch Verkürzung entstandenen Deviant war. Die Größenverhältnisse der MMNs drehten sich um, wenn dieselbe Manipulation der Dauer an vokalischen Stimuli vorgenommen wurde. Da weder die spektrale Reduktion des Vokals noch eine spektrale Anreicherung der Sinustöne das Verhältnis umkehren konnten, führten die Autoren die offenbar unterschiedlichen Verarbeitungsmodi auf die Sprachlichkeit des Materials zurück. Unbeantwortet bleibt die Frage, wodurch sich „Sprachlichkeit“ definiert.

Aber auch andere Autoren gehen von der Existenz spezifisch sprachlicher Verarbeitungswege aus und halten besonders diese bei der Lese-/Rechtschreibschwäche für unterentwickelt.

$\mathrm{Zu}$ den Vertretern dieser Position zählen beispielsweise Schulte-Körne, Deimel, Bartling und Remschmidt (1998c, 1999b). Die im Kontext des temporalen Defizits erwähnten Befunde der Arbeitsgruppe lassen sich auch im Sinne eines sprachspezifischen Verarbeitungsdefizits lese/rechtschreibschwacher Personen deuten, wie es die Arbeitsgruppe selbst tut. Das Marburger Team hatte ebenso wie Mody (1993) und Mody et al. (1995) bei lese-/rechtschreibschwachen Jungen im Vergleich zu gesunden Jungen eine Reduktion der MMN-Amplitude für den sprachlichen Kontrast /ba/ versus /da/, nicht aber für den Frequenzkontrast zweiter Sinustöne (1000 Hz versus $1050 \mathrm{~Hz}$ ) nachweisen können. 
Der mithilfe der MMN neurophysiologisch detektierbare Gruppenunterschied in der Diskrimination des /ba/ - /da/ - Kontrastes fand bei Schulte-Körne, Deimel, Bartling und Remschmidt (1999b) weiteren Niederschlag in einem LISREL-Modell, dessen basalste Komponente der elektrophysiologische Indikator darstellt. Das Modell versucht, die Fortpflanzung des Verarbeitungsdefizits von der neurophysiologischen präattentiven Ebene über die Ebenen der behavioralen Diskrimination und der phonologischen Bewusstheit bis schließlich hin zur Schreibschwäche zu verfolgen (vgl. Abbildung 3).

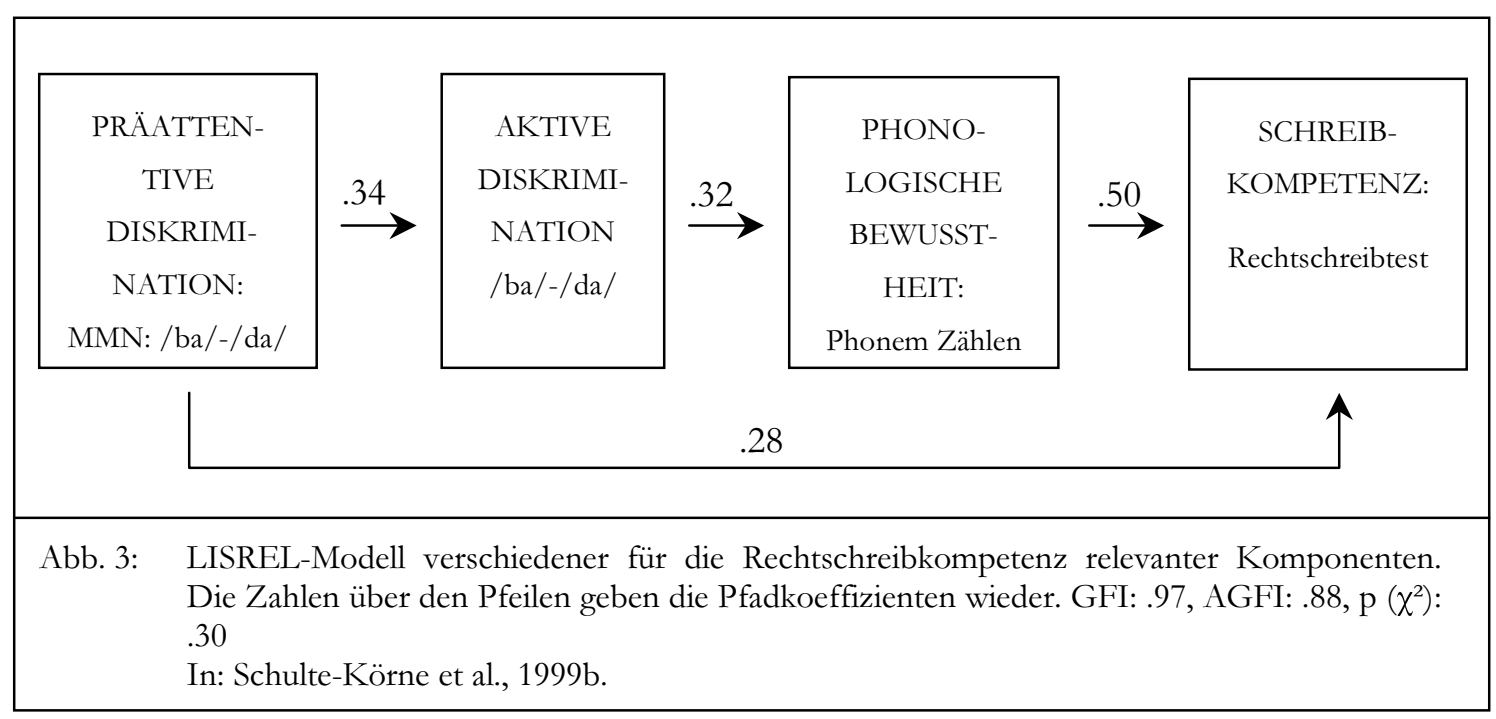

Die aktive Diskrimination wurde anhand der behavioralen Unterscheidung derselben Stimuli umgesetzt, die auch im EEG-Paradigma den Kontrast konstituierten; zur Erfassung der phonologischen Bewusstheit mussten die Probanden die Phoneme einzelner Wörter zählen; die Schreibkompetenz wurde durch einen altersangepassten Rechtschreibtest gemessen. Noch weitere Parameter wie die nichtsprachliche Diskriminationsfähigkeit (anhand von Sinustönen) und eine Aufgabenstellung zur raschen temporalen Verarbeitung hatten als potentielle Komponenten des Modells zur Verfügung gestanden, doch korrelierten diese nicht mit schriftsprachlicher Kompetenz.

Desweiteren ist zu bemerken, dass Zusammenhänge mit der Lesekompetenz jeweils nicht signifikant ausfielen.

Durch sein Muster korrelativer Einschlüsse ebenso wie durch das Muster nicht eingeschlossener Komponenten trifft das Modell von Schulte-Körne et al. (1999b) eine gewichtige Aussage über die Bedeutsamkeit der genannten Komponenten für die Schreibfähigkeit.

Obwohl Defizite auf nonverbaler Ebene in den Hintergrund rücken, besteht die Hauptaussage der Autoren, die die Position eines sprachspezifischen Verarbeitungsdefizits bei lese-/recht- 
schreibschwachen Personen vertreten, nicht darin, diese nichtsprachlichen Defizite zu negieren. Die Verursachung der Schwierigkeiten im Schriftspracherwerb wird jedoch hauptsächlich einer Schwäche der spezifisch sprachlichen Verarbeitung zugeschrieben.

Auch Hinweise Csépe und Gyurkócza (1995) interpretierten ihre Befunde im Sinne eines spezifisch sprachlichen Defizits: Sie untersuchten die Diskrimination von (bezüglich Artikulationsort und Stimmhaftigkeit differierender) Konsonanten (/ba/ versus /ga/ bzw. /pa/), die Diskrimination von Vokalen (/e/ versus /i/ bzw. /ø/) sowie die Diskrimination von Sinustönen (1000 versus $1050 \mathrm{~Hz}$ bzw. $1200 \mathrm{~Hz}$ ) bei lese-/rechtschreibschwachen und sprachunauffälligen Kindern. Für jeden Kontrast wurden die Amplitude und die Latenz ausgewertet. Signifikante Gruppenunterschiede traten bei allen Konsonanten-Kontrasten sowie bei den MMN-Amplitude des Stimuluspaars /e/ - /i/ und des Sinustonpaars 1000 versus 1050 $\mathrm{Hz}$ auf.

Studdert-Kennedy und Mody (1995) nehmen eine Zwischenposition zwischen der Favorisierung phonologischer Defizite und der Vermutung von Schwierigkeiten der Verarbeitung rasch aufeinanderfolgender Reize ein. Seine Haltung beruht u.a. auf Experimenten zur kategorialen Wahrnehmung (z.B.: De Weirdt, 1988; Steffens, Eilers, GrossGlen \& Jallad, 1992; Godfrey, Syrdal-Lasky, Millay \& Knox, 1981; Werker \& Tees, 1987). Die besondere Schwierigkeit lese-/rechtschreibschwacher Personen besteht demnach weniger im Erkennen akustischer Unterschiede innerhalb einer phonologischen Kategorie, sondern in der Diskrimination zwischen diesen Kategorien. Diese Identifikationsprobleme könnten durch unscharfe kategoriale Langzeitmuster für phonologische Kategorien zustande gekommen sein (Studdert-Kennedy \& Mody, 1995). An dieser Stelle sind die Untersuchungsreihen von Mody (1993) bzw. Mody et al. (1995) anzuführen, die die Hypothese des raschen temporalen Verarbeitungsdefizits hinterfragen sollten. Mit den Stimuli /ba/ und /da/ wurden die Paradigmata nachgestellt, die Tallal (1980) zur Formulierung ihrer Hypothese geführt hatten, nämlich das „TOJ“ und das „same / different“ - Paradigma. Je kürzer das ISI eingestellt wurde, desto stärker häuften sich erwartungsgemäß Diskriminationsfehler lese-/rechtschreibschwacher Kinder. Tallal würde zur Begründung dieser Befunde die schnellen Transitionen der Stop-Konsonanten heranziehen. Dem widersprach jedoch, dass im Folgeexperiment Sinustöne mit Transitionen auf einem zweiten und dritten Formanten versehen wurden, die denen der Konsonanten entsprachen, die Diskriminationsleistung der lese-/rechtschreibschwachen Kinder gegenüber gesunden Kindern sich jedoch nicht verschlechterte. Allerdings wurde ebenso der Verdacht ausgeräumt, die pure Sprachlichkeit bedinge den Leistungseinbruch lese/rechtschreibschwacher Kinder bei der Differenzierung der Stop-Konsonanten: Der Kontrast 
/sa/ versus / $\mathrm{a}$ / wurde von Kindern beider Experimentalgruppen gleich gut bewältigt. Die Arbeitsgruppe konkretisierte infolgedessen den phonologischen Kern ihrer These: Das Verarbeitungsdefizit betreffe die Diskrimination eng benachbarter phonologischer Kategorien und würde erst bei höheren Ansprüchen an die Verarbeitungsgeschwindigkeit deutlich. 


\section{EIGENE FRAGESTELLUNG}

Die vorangegangenen Abschnitte stellten eine Reihe konvergierender Ergebnisse vor: Zwei Forschungstraditionen mit völlig unterschiedlichen Herangehensweisen können Befunde vorlegen, die jeweils mit der Lese-/Rechtschreibstörung in Verbindung stehen. Allein diese Tatsache motiviert bereits dazu, in einem Brückenschlag zwischen diesen beiden herauszufinden, ob und inwiefern die beiden prädiktorischen Bereiche zusammen hängen. Zusätzlich erhärtet der Vergleich konkreter Merkmale von Parametern des EEG-Bereichs mit einem Konstrukt der phonologischen Verarbeitung, nämlich dem des phonologischen Arbeitsgedächtnisses, den Verdacht eines Zusammenhangs zwischen kognitionspsychologischen und neurophysiologischen Verarbeitungsdefiziten bei Personen mit Lese-/Rechtschreibschwäche:

Im auditiven Gedächtnis sowie beim Arbeitsgedächtnis ist die Rede von einem passiven Speicher für rezente akustische Information mit automatischem primärem Zugang. Die Speicherkapazität ist begrenzt. Sowohl hierin als auch hinsichtlich der Tatsache, dass diese Limitation durch den Faktor Zeit (anstelle von Speichermasse) bestimmt wird, sind die Modelle kongruent. Die Angaben über die Überlebensdauer der Speicherinhalte bewegen sich für beide Konstrukte im Sekundenbereich, was den Verdacht von Kongruenz der Konstrukte weiter verstärkt.

Die Spuren der eingetroffenen akustischen Eigenschaften erzeugen eine diese integrierende Repräsentation, mutmaßen Näätänen und Winkler (1999). Die Vorrichtungen in Baddeleys Arbeitsgedächtnismodell speichern die Gedächtnisspuren zwar nur noch (phonetischer Speicher) bzw. erhalten sie aufrecht (Rehearsal); möglicherweise handelt es sich dabei jedoch um diejenigen Spuren, die das sensorische Gedächtnis vorher gebildet hat. Dies wäre mit der Vorstellung konsistent, dass das sensorische auditive Gedächtnis einen Vorläufer des Arbeitsgedächtnis darstellt.

Die Gedächtnisspuren stehen in reziproker Verbindung zu Spuren im Langzeitgedächtnis. Dies behaupten ebenfalls Vertreter beider Modellvorstellungen. Cheour, Čeponiene, Lehtokoski, Luuk, Allik, Alho, \& Näätänen (1998) beispielsweise demonstrierten sehr eindrücklich die Entstehung von Langzeitrepräsentationen auf der Basis des auditorischen Einflusses und deren Rückwirkung auf die Diskriminationsfähigkeit innerhalb des sensorischen Gedächtnisses. Derartige Interaktionen zwischen phonologischer Schleife und Wissensbasis sieht auch das Modell von Baddeley vor. 
Bezüglich des Formats der aufgenommenen auditorischen Information lassen sich ebenfalls Gemeinsamkeiten zwischen auditivem sensorischen und Arbeitsgedächtnis finden: Jedes Merkmal, das Teil des Spektrums akustisch wahrnehmbarer Eigenschaften ist -von der Tonhöhe über dynamische Eigenschaften bis hin zu phonetischen Kriterien-, ist in der Lage, eine Mismatch Negativity auszulösen. All diese Merkmale finden folglich Eingang in den auditiven sensorischen Speicher. Für den phonetischen Speicher im Arbeitsgedächtnis scheinen nicht mehr alle, aber noch ein Ausschnitt dieser Eigenschaften relevant zu sein. Hier liegt der Fokus v.a. auf der „Sprachlichkeit“ der Information (Baddeley \& Salamé, 1986) wobei jedoch wichtig anzumerken ist, dass Sprache das Aggregat der Einzeleigenschaften darstellt. Neben der Sprachlichkeit werden im phonologischen Arbeitsgedächtnis auch weiterhin die temporalen Merkmale codiert, wie Klatte und Hellbrück (1993), Jack (1990) oder Grube (1999) zeigten.

Zudem weisen Aussagen über die Prinzipien der Codierungsmodi beider Speichersysteme eine bemerkenswerte Koinzidenz auf: Näätänen wies den Generationsprozess der MMN als Änderungsdetektionsprozess im auditiven sensorischen Gedächtnis aus (vgl. Näätänen \& Winkler, 1999). Jones und Macken (1993) kamen bei Untersuchungen zum Arbeitsgedächtnis zu dem Schluss, für den Zutritt zur phonologischen Schleife sei weniger der Zustand des akustischen Materials selbst als vielmehr die Art seiner Zustandsveränderung bedeutsam (changing state hypothesis). Parallelen lassen sich möglicherweise auch aus der Codierungsqualität von Änderungen ziehen: Wenn zwischen der Diskriminationsfähigkeit und der Intensität der Mismatch Negativity - Reaktion eine Korrelation festgestellt werden konnte (Tiitinen, Sinkkonen, May \& Näätänen, 1994), weist dies den Parameter auch als ein Maß für die Diskriminationspräzision aus - als ein wichtiges Funktionsmerkmal des phonetischen Speichers formulierten Hasselhorn, Grube und Weiß (Grube, Hasselhorn \& Weiß, 1998; Hasselhorn, 1999) die Dimension „Präzision“.

Ausgangspunkt für einen weiteren Verknüpfungspunkt bilden die Arbeiten von Gathercole und Baddeley (Gathercole \& Baddeley, 1993; Baddeley et al., 1998) zur phonologischen Schleife. Die Autoren schrieben ihr eine Schlüsselrolle beim Wortschatzerwerb sowohl in einer Fremdals auch in der Muttersprache zu. Cheour et al. (1998) zeigten im auditiven sensorischen Gedächtnis die Bedeutung des Mismatch-Prozesses für die Wiedererkennung von Phonemen der Muttersprache; Winkler et al. (1999) demonstrierten das Gleiche für Phoneme einer Fremdsprache. Führt man sich vor Augen, dass der Erwerb von Worten auf dem Erwerb von Phonemen basiert, so stellen sich die Ergebnisse so dar, als hätten Cheour und Winkler die neurophysiologischen Prozess-Voraussetzungen für die Arbeit der phonologischen Schleife aufgezeichnet, die Gathercole und Baddeley als so essentiell für den Spracherwerb erkannten. 
Den einzigen experimentellen Vorstoß in Richtung einer Verknüpfung der beiden Ebenen wagten Čeponiene, Service, Kurjenluoma, Cheour und Näätänen (1999). Sie bestimmten aus einer Gruppe sieben- bis neunjähriger (im Schriftspracherwerb unauffälliger) Kinder anhand des Kunstwort-Nachsprechens zwei Extremgruppen. Deren Leistungsunterschiede beim Kunstwort-Nachsprechen spiegelten sich in einem Gruppenunterschied der MMN während der passiven Diskrimination von /baga/ versus /baka/ wieder. Darin sah das finnische Team ein Indiz dafür, dass Unterschiede im phonologischen Arbeitsgedächtnis -wie sie durch das Kunstwort-Nachsprechen erfasst werden- mit Unterschieden im auditiven sensorischen Gedächtnis -wie sie die MMN reflektiert- in enger Verbindung stehen.

Aus den theoretischen Überlegungen sowie aus der eben genannten Studie wird ersichtlich, dass Verbindungen zwischen der Ebene neurophysiologischer Parameter und der Ebene kognitionspsychologischer Konzepte durchaus plausibel sind. Dabei stellen die einzelnen mit unterschiedlichen Parametern sichtbar gemachten Verarbeitungsdefizite möglicherweise unterschiedliche Zeitpunkte und Stadien ein und derselben defizitären Verarbeitung dar. Dies entspräche einer in der Literatur häufig geäußerten Vorstellung (z. B. Csépe \& Gyurkócza, 1995), die Lese-/Rechtschreibschwäche basiere auf insuffizienten Phonem- bzw. WortRepräsentationen.

Diese Verbindungen zwischen neurophysiologischen und kognitionspsychologischen Verarbeitungsdefiziten bei Lese-/Rechtschreibschwäche aufzudecken und zu spezifizieren, ist das Hauptziel der vorliegenden Studie. Dieses Hauptziel wird über drei spezifischere Fragestellungen zu erreichen versucht, die in den folgenden Abschnitten aufgeführt werden. 


\subsection{PHONOLOGISCHE VERARBEITUNGSDEFIZITE VON KINDERN MIT LESE-/RECHTSCHREIBSCHWÄCHE (KOGNITIONSPSYCHOLOGISCHE EBENE)}

Um einen möglichen Zusammenhang zwischen neurophysiologischen und kognitionspsychologischen Verarbeitungsdefiziten bei Lese-/Rechtschreibschwäche aufzudecken, ist es zunächst nötig, die neurophysiologischen und kognitionspsychologischen Verarbeitungsdefizite nachzuvollziehen. Ein erster Abschnitt widmet sich den kognitionspsychologischen Schwierigkeiten. Aus dem bisher Gesagten geht die Erwartung hervor, dass die kognitionspsychologische Verarbeitung bei lese-/rechtschreibschwachen Kindern beeinträchtigt ist (psychologische Hypothese $(\mathrm{pH})$ 1.0). Dies würde im Hinblick auf die Triade phonologischer Verarbeitung von Wagner und Torgesen (1987; bzw. Wagner, Torgesen \& Rashotte, 1994) bedeuten, dass das phonologische Arbeitsgedächtnis ( $\mathrm{pH}$ 1.a [pH 1 zum Arbeitsgedächtnis]), die phonologische Bewusstheit ( $\mathrm{pH}$ 1.b [ $\mathrm{pH} 1$ zur phonologischen Bewusstheit]) und der Abruf aus dem Langzeitgedächtnis ( $\mathrm{pH} 1.1$ ( $\mathrm{pH} 1$ zum Abruf aus dem Langzeitgedächtnis]) funktionsgestört sind. Die Verarbeitungsschwäche schlösse alle Konstrukt-Untereinheiten -soweit vorhandenein: Für das phonologische Arbeitsgedächtnis beträfe die Erwartung einer Beeinträchtigung demzufolge den phonetischen Speicher mit den Funktionsmerkmalen Speicherkapazität, Speicherpräzision und Rehearsalgeschwindigkeit; für die phonologische Bewusstheit lese/rechtschreibschwacher Kinder würde eine verschlechterte phonologische Bewusstheit im engeren Sinne wie auch eine verschlechterte phonologische Bewusstheit im weiteren Sinne erwartet werden.

\subsection{SENSORISCH AUDITORISCHE VERARBEITUNGSDEFI- ZITE VON KINDERN MIT LESE-/RECHTSCHREIB- SCHWÄCHE (NEUROPHYSIOLOGISCHE EBENE)}

Für das auditive sensorische Gedächtnis werden ebenfalls Beeinträchtigungen bei lese/rechtschreibschwachen Personen erwartet ( $\mathrm{pH}$ 2.0). Diese Verarbeitungsdefizite auf der neurophysiologischen Ebene sollen in einem zweiten Abschnitt nachvollzogen werden. 
Zur Verursachung der Lese-/Rechtschreibschwäche durch ein Defizit im auditiven sensorischen Gedächtnis existieren verschiedene differenziertere konkurrierende Hypothesen. Die drei gewichtigsten sollen im Fokus der vorliegenden Untersuchung stehen. Es handelt sich hierbei um die Hypothese einer Schwäche der Verarbeitung rasch temporaler Reize ( $\mathrm{pH} 2 . \mathrm{t}$ ), die Hypothese einer generellen auditiven Verarbeitungsstörung ( $\mathrm{pH}$ 2.g) und die Hypothese einer Schwäche der spezifisch sprachlichen Verarbeitung ( $\mathrm{pH}$ 2.s). Da die Hypothesen konkurrieren, besteht keine Erwartung im Sinne einer Favorisierung eines bestimmten Typs sensorisch auditiven Verarbeitungsdefizits; vielmehr richtet sich die Erwartung auf Verarbeitungsdefizite im Sinne einer (mindestens einer - sie schließen sich nicht unbedingt wechselseitig aus) der drei Hypothesen.

Welchem Defizittypus eventuelle auftretende Verarbeitungsschwierigkeiten zuzuordnen sein werden, wird möglicherweise Rückschlüsse bezüglich der Richtigkeit der einen oder anderen Hypothese erlauben. Derartige Rückschlüsse stellen ein Nebenziel der Studie dar.

\subsection{ZUSAMMENHANG ZWISCHEN PHONOLOGISCHEN UND AUDITIV-SENSORISCHEN VERARBEITUNGSDEFIZITEN}

Wenn die kognitionspsychologischen und neurophysiologischen Verarbeitungsdefizite lese/rechtschreibschwacher Personen nachvollzogen werden können, kann der Zusammenhang bei der Vorhersage von Lese-/Rechtschreibschwierigkeiten zwischen beiden Bereichen fokussiert werden. Gemäß der Erläuterungen zu Eingang dieses Abschnitts besteht eine klare Erwartung hinsichtlich des Vorhandenseins eines Zusammenhangs ( $\mathrm{pH}$ 3a). Desweiteren soll dieser spezifiziert werden. Die Exploration findet in zwei Schritten statt: Zum einen gilt es, gegenseitige Abhängigkeiten der Verarbeitungsebenen bzw. der Konstrukte auszumachen und deren Größenordnung zu ermitteln. Dies schließt die Abschätzung des Erklärungspotenzials jeder einzelnen Ebene bzw. jeden einzelnen Konstrukts ein. Ebenso stellt sich die Frage, inwieweit die neurophysiologische Ebene Erklärungspotenzial der schriftsprachlichen Leistung bietet, welches vom Erklärungspotenzial der phonologischen Verarbeitungsebene unabhängig ist.

In einem darauf aufbauenden Schritt soll der Zusammenhang modelliert werden. Die entsprechenden Erwartungen ( $\mathrm{pH} 3 \mathrm{~b}$ ) für Beeinflussungswege der Konstrukte sind in Abbildung 4 veranschaulicht. 


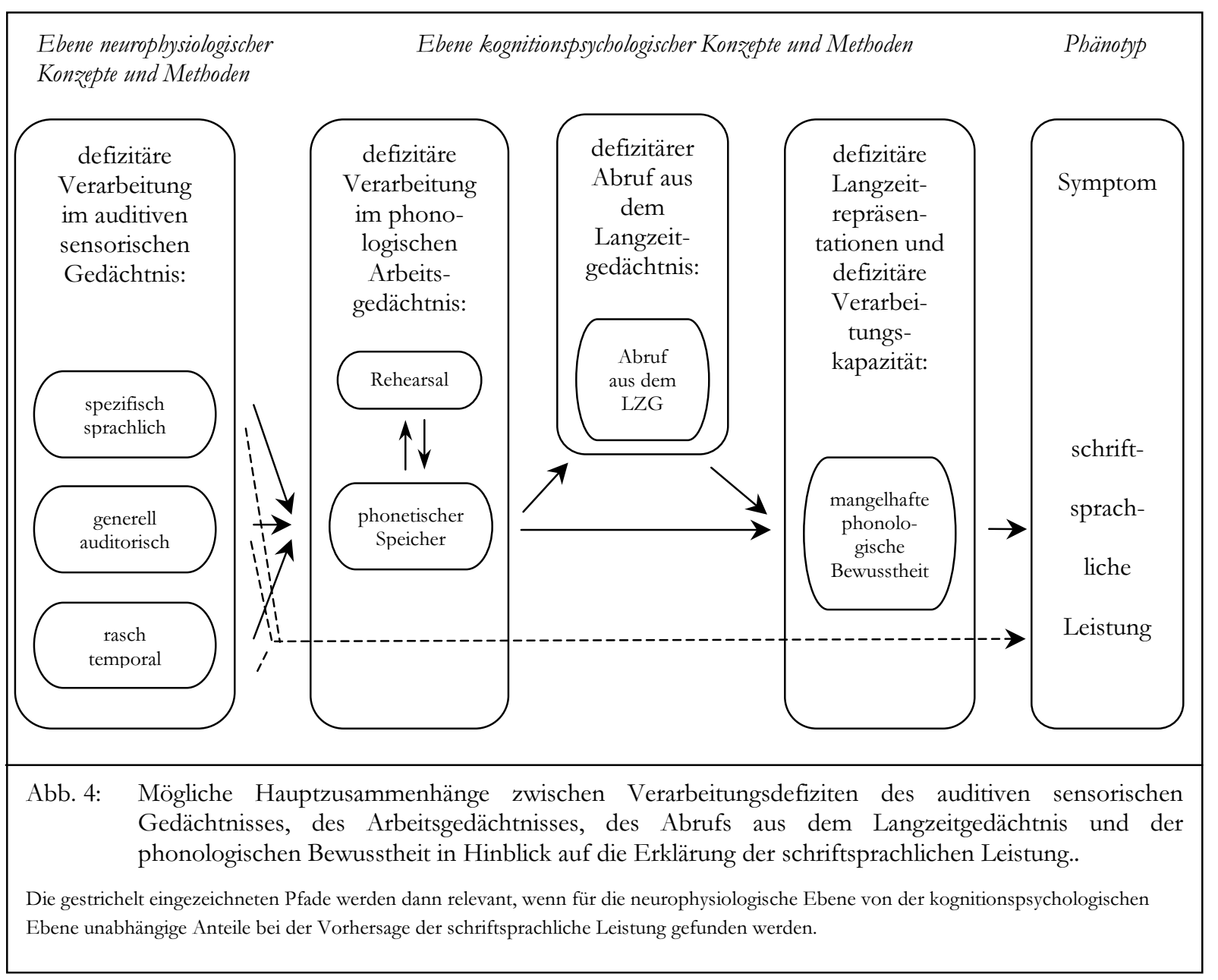

Die erste Strukturierung für die Modellerwartung geben die Angaben der Literatur für die zeitlichen Rahmen der Aktivierung bestimmter Gedächtniseinheiten: Die Abrufbarkeit von Inhalten des auditiven sensorischen Gedächtnis endet bereits nach wenigen Sekunden (Broadbent, 1958, 1984). Die Aktivitätsdauer des Kurzzeitgedächtnisses, zu welchem die Operationen des Arbeitsgedächtnisses, der Abruf aus dem Langzeitgedächtnis und die Aktivitäten im Rahmen der phonologischen Bewusstheit gezählt werden, liegt hingegen im Minutenbereich. Die daraus abzuleitende Schlussfolgerung wäre, dass die neurophysiologischen Prozesse allen kognitionspsychologischen Prozessen vorausgehen.

In Anlehnung an das Modell von Schulte-Körne et al. (1999b) wird auch eine direkte Vorhersage der neurophysiologischen Ebene auf die schriftsprachliche Leistung eingeräumt. Diese basiert auf der Existenz von der phonologischen Verarbeitung unabhängiger Anteile der 
neurophysiologischen Verarbeitung bei der Vorhersage der schriftsprachlichen Leistung und wird daher als mögliche Erwartung betrachtet.

Durch die frappierende Ähnlichkeit zwischen den Eigenschaften des phonetischen Speichers und Merkmalen der auditiv-sensorischen Gedächtnis-Parameters der MMN (Punkt 3.) -wobei hier insbesondere die sehr kurze Behaltensdauer des phonetischen Speichers (1.5-2 sek: Baddeley, 1990) zu erwähnen ist- nimmt das Arbeitsgedächtnis eine besondere Rolle im Kreise der kognitionspsychologischen Konstrukte ein. Verarbeitungsergebnisse des auditiven sensorischen Gedächtnisses werden mit der höchsten Wahrscheinlichkeit zuerst in das Arbeitsgedächtnis eingelesen. Im Modell ist es daher sicherlich an die Stelle nach dem auditiven sensorischen Gedächtnis zu setzen.

Die phonologische Bewusstheit konnte sich nicht nur für den Erwerb der Lesefähigkeit, sondern auch für das Schreibenlernen als starker Prädiktor des Schriftspracherwerbs bestätigen (Lundberg et al., 1980). Es ist daher davon auszugehen, dass diese den Anforderungen bei schriftsprachlichen Leistungen näher steht als die anderen beiden kognitionspsychologischen Konstrukte. Die phonologische Bewusstheit erhält im Modell demzufolge die Position vor der schriftsprachlichen Leistung.

Der Einfluss des Arbeitsgedächtnisses auf die schriftsprachliche Leistung (Gathercole, 1997; Gathercole, Willis, Emslie \& Baddeley, 1992) muss infolgedessen im Kontext der vorliegenden Konstrukte indirekter Natur sein, das heißt, über die phonologische Bewusstheit führen.

Wie in Punkt 2.3.3.2. angedeutet, steht der Abruf aus dem Langzeitgedächtnis vermutlich sowohl mit dem Arbeitsgedächtnis als auch mit der phonologischen Bewusstheit in engem Zusammenhang. Das Arbeitsgedächtnis steuert möglicherweise mit seiner Regulationseinheit, der Zentralen Exekutive, den Langzeitgedächtnis-Abruf; eine leistungsstarke Aktivierung unterstützt möglicherweise bei den Manipulationsaufgaben zur phonologischen Bewusstheit. Die entsprechende Eingliederung des Abrufs aus dem Langzeitgedächtnis in das Modell geschähe demzufolge als ein vom Arbeitsgedächtnis beeinflusstes und die phonologische Bewusstheit beeinflussendes Konstrukt.

Die bis hierhin abgeleiteten Pfade stellen die wichtigsten Zusammenhänge in dem vorhandenen Kontext dar. Wichtig zu betonen bleibt, dass in der Realität von einem weit engmaschigeren Netz auch wechselseitiger Einflüsse der Komponenten auszugehen ist. Ein Modell ist jedoch nur prüfbar, wenn es lediglich die Hauptpfade enthält. 


\section{METHODE}

\subsection{DESIGN}

Um phonologische Verarbeitungsdefizite lese-/rechtschreibschwacher Personen zu identifizieren und gegebenenfalls miteinander in Verbindung $\mathrm{zu}$ bringen, bietet sich ein Parallelgruppen-Design an: Einer Gruppe lese-/rechtschreibschwacher Probanden sowie einer alters-, geschlechts- und intelligenzgematchten unauffälligen Vergleichsgruppe werden Aufgaben gestellt, die mögliche Defizite berühren sollen. Die Aufgaben entstammen den bereits im Verdacht defizitärer Verarbeitung stehenden Bereichen, nämlich der phonologischen Bewusstheit, dem Arbeitsgedächtnis, dem Abruf aus dem Langzeitgedächtnis und dem auditiven sensorischen Gedächtnis.

\subsection{UNABHÄNGIGE VARIABLEN}

\subsubsection{DEFINITION DER GRUPPENZUGEHÖRIGKEIT}

Untersuchungen zur Lese-/Rechtschreibstörung sind ab einem Alter sinnvoll, ab dem die Störung diagnostizierbar ist und während einer Periode, in der die schriftsprachliche Schwäche die aktuellen Leistungsanforderungen noch massiv beeinträchtigt, da eine Therapie noch nicht begonnen bzw. nicht erfolgreich abgeschlossen wurde. Der Beginn der Diagnostizierbarkeit liegt in etwa beim Eintritt in die zweite Klasse, wenn gewisse Grundkenntnisse im Lesen und Schreiben bereits vorausgesetzt werden dürfen. Zur Diagnose kommt es häufig erst im Verlauf der dritten Klasse. Die sich anschließende Therapie kann mehrere Jahre in Anspruch nehmen. Aufgrund dieser Überlegungen wurde für die vorliegende Studie ein Altersspektrum von 8;0 bis 13;11 Jahren ausgewählt.

Die Probanden entstammten einer Inanspruchnahmepopulation der Poliklinik der Kinder- und Jugendpsychiatrie des Universitätsklinikums Göttingen.

Den Ankerpunkt der Diagnose einer Lese-/Rechtschreibschwäche bildete, wie es im deutschen Sprachraum üblich ist, die Rechtschreibleistung. Diese durfte einen T-Wert von 40 (Prozentrang 17) nicht überschreiten und sollte gleichzeitig eine Diskrepanz von mindestens 12 
T-Punkten zur höheren Intelligenz aufspannen. Die Grenze für die kritische Rechtschreibleistung fiel hiermit um 3 T-Punkte (7 Prozentränge) milder aus als es die Forschungskriterien von ICD 10 oder DSM IV erfordern würden. Mit dieser Festlegung wurde jedoch dem diagnostischen Verfahren im klinischen Alltag Rechnung getragen.

Obwohl die orthographische Leistung diejenige Teilleistung sein sollte, welche über die Gruppenzuordnung entschied, sollte der andere Aspekt der schriftsprachlichen Schwäche, die Leseproblematik, ebenfalls bestimmt werden, um sicherzustellen, dass die Bezeichnung „Lese/Rechtschreibschwäche“ für die selegierte klinische Gruppe wirklich gerechtfertigt ist.

Uneindeutiger bleibt dabei die Definition für „keine Lese-/Rechtschreibschwäche“: Probanden, auf die nur eines der beiden diagnostischen Kriterien zutrifft, ist korrekterweise keine Lese/Rechtschreibschwäche zu diagnostizieren. Wirklich „gesund“ hinsichtlich des Schriftspracherwerbs können jedoch nur Personen jenseits beider Kriterien genannt werden. Als Kontrollproband eignete sich demzufolge, wer keine der beiden Komponenten des Doppelkriteriums aufwies, das heißt, mindestens durchschnittliche orthographische Fähigkeiten demonstrierte, und dieses Leistungsniveau mit seiner Intelligenz um nicht mehr als 12 T-Punkte überschritt.

Die Sinnhaftigkeit der Diagnostik der Lese-/Rechtschreibstörung anhand des Doppelkriteriums von Intelligenz und Lese-/Rechtschreibleistung wird immer wieder in Frage gestellt. Einen kurzen Einblick in die Debatte soll der folgende Exkurs vermitteln: 


\subsubsection{EXKURS: PROBLEMATIK DER DISKREPANZDEFINITION}

Der Einbezug des Intelligenzkriteriums hat zur Folge, dass bei gleicher schriftsprachlicher Leistung die Wahrscheinlichkeit einer -therapieentscheidenden- Diagnose abnimmt, je größer die Distanz zum Intelligenzdurchschnittswert 100 wird. Das ist im obigen Koordinatensystem leicht nachzuvollziehen. Von besonderer therapeutischer Bedeutsamkeit ist diese Separation für die Gruppe der schlechten Rechtschreiber. Die diagnostische Differenzierung gründet sich auf das Verständnis der Lese-/Rechtschreibschwäche als Teilleistungsschwäche und der damit verbundenen Implikation, im Falle einer mit den intellektuellen Möglichkeiten inkonsistenten Schreibstörung lägen andere basale kognitive Defizite vor als dies bei einer schriftsprachlichen Schwäche der Fall wäre, die aufgrund des intellektuellen Leistungsniveaus vorhersagbar ist. Dies wurde jedoch nie bestätigt. Statt dessen konnten viele Forschergruppen zeigen, dass nicht nur die schriftsprachlichen Fähigkeiten schlechter Rechtschreiber bzw. lese-/rechtschreibschwacher Probanden miteinander vergleichbar waren, sondern offenbar auch Komponenten der phonologischen Verarbeitung gleichermaßen betroffen waren. Zu nennen wären hier das Kunstwort-Nachsprechen (Jorm, Share, MacLean \& Matthews, 1986; Bloom, Wagner, Reskin \& Bergman, 1980), die Sprechrate (Das, Mensink \& Mishra, 1990), Aufgaben zum Kurzzeitgedächtnis, zum phonetischen Rekodieren (Klicpera \& Gasteiger-Klicpera, 2001) und schließlich zur phonologischen Bewusstheit (Taylor, Satz \& Friel, 1979). In allen Fällen lagen die Leistungen der schlechten Rechtschreiber ebenso wie die der Lese-/rechtschreibschwachen deutlich hinter denen der gesunden Probanden zurück, unterschieden sich aber untereinander nicht. Nicht einmal im Fehlerprofil der Rechtschreibung konnten signifikante Gruppenunterschiede identifiziert werden (Klicpera \& Gasteiger-Klicpera mit Schabmann, 1993). Diejenigen Gruppenunterschiede, die zwischen den beiden lese-/rechtschreibschwachen Populationen gefunden werden, betrafen nicht die phonologische Verarbeitung. Es handelte sich beispielsweise um Unterschiede im orthographischen Wissen (Foorman, Francis, Fletcher \& Lynn, 1996; Siegel, Share \& Geva, 1995) oder der rechnerischen Befähigung (Graham \& Kline, 1991; Silva, McGee \& Williams, 1985). Diese Leistungen fielen jeweils zugunsten der intelligenzdiskrepanten Gruppe aus und ließen sich folgerichtig durch deren Intelligenzvorteil erklären (Klicpera \& Gasteiger-Klicpera, 2001).

$\mathrm{Zu}$ diesen empirischen Daten kommen theoretische Überlegungen, die die Diskrimination der nicht intelligenzdiskrepanten von der intelligenzdiskrepanten lese-/rechtschreibschwachen Personengruppe weiterhin in Frage stellen. Zum einen ist anzuführen, dass keine instrumentelle Richtlinie zur Erhebung der Intelligenz existiert, auf deren Basis die Diskrepanz zu berechnen 
wäre. Damit beruht das diagnostische Ergebnis auch auf der individuellen Forscherentscheidung für einen leichteren oder schwereren Test bzw. für die eine oder andere Test-Unterskala.

Eine weitere Überlegung betrifft die bei der Diagnostik mittels Diskrepanzkriterium vorausgesetzte Vorhersagbarkeit der Rechtschreibleistung auf der Grundlage des Intelligenzniveaus: Besonders in den Extrembereichen der Intelligenz-Skala besteht keine hohe Korrelation zur Rechtschreibleistung; Hochbegabte zeigen nicht unbedingt außerordentliche Leistungen im Rechtschreiben, und gering begabte Menschen schreiben oft deutlich besser, als es ihrem Intelligenzniveau entsprechen würde. Aufgrund dieser Problematik sahen sich Schulte-Körne, Deimel \& Remschmidt (2001) sogar veranlasst, -inspiriert durch das Vorbild englischsprachiger Literatur, wo häufig regressionsanalytisch berechnete kritische Werte den absoluten Diskrepanzwert ersetzen (Evans, 1990)-, eine Zuordnung kritischer Prozentrangwerte der Rechtschreibung zu Intelligenzbereichen zu erstellen.

Wie es die oben geschilderten Befunde und Überlegungen deutlich machen, wird die Sinnhaftigkeit des Intelligenzkriteriums für die Lese-/Rechtschreibschwäche-Diagnose bzw. die Sinnhaftigkeit einer Differenzierung der im Lesen und Schreiben leistungsschwachen Personen in eine nichtdiskrepante Gruppe nicht therapieberechtigter schlechter Rechtschreiber und in eine diskrepante therapieberechtigte Gruppe Lese-/rechtschreibschwacher mittlerweile von vielen Seiten in Frage gestellt.

\subsubsection{INSTRUMENTE ZUR ERHEBUNG DER GRUPPENZUGEHÖRIGKEIT}

$\mathrm{Zu}$ ermittelnde Kennwerte zur Feststellung der Gruppenzugehörigkeit (bzw. zur Deskription der Stichprobe) betreffen die Intelligenz, die Rechtschreibleistung, die Diskrepanz zwischen diesen beiden und schließlich die Lesekompetenz.

Zur Erhebung der Intelligenz wurde der Hamburg-Wechsler-Intelligenztest für Kinder HAWIK-III (Tewes, Rossmann \& Schallenberger, 1999) ausgewählt. Dieser setzt sich aus zehn Unter- und drei Zusatztests (jeweiliger Skalenmittelwert = 10; s = 2) zusammen, die sich nicht nur zu einem Gesamt-Intelligenzquotienten zusammenfügen, sondern sich außerdem einem Teilbereich verbaler bzw. einem Teilbereich handlungsbezogener Fähigkeiten zuordnen lassen (Skalenmittelwert der Quotienten $=100 ; \mathrm{s}=15)$. Der Gesamt-Intelligenzquotient diente zur Bestimmung des Diskrepanzkriteriums; anhand der Teilskala des Handlungs-Intelligenzquotienten wurde die Parallelisierung der Gruppen vorgenommen. Es ist bei Untersuchungen zur 
Lese-/Rechtschreibschwäche allgemein üblich, nach praktischer Intelligenz zu parallelisieren, da im Verbalbereich bei lese-/rechtschreibschwachen Kindern Einbußen auftreten können, die möglicherweise dem Störungsbild zuzuschreiben sind. Diese könnten beispielsweise durch Untertests des Verbalteils zu erklären sein, bei denen die schlechtere kurzfristige Behaltensleistung im Arbeitsgedächtnis (de Jong, 1998; Gathercole, 1997; Gathercole et al., 1992), die größeren Schwierigkeiten beim Wortschatzerwerb (Baddeley et al., 1998; Gathercole \& Baddeley, 1993) oder auch die größere Schwierigkeit des Zugriffs auf die semantische Wissensbasis lese-/rechtschreibschwacher Personen eine Rolle spielen.

Mit einem jeweils altersangemessenen deutschen Rechtschreibtest für Kinder (Diagnostischer Rechtschreibtest: DRT 4 von Grund, Haug, Naumann \& 1994; DRT 5 von Grund, Haug \& Naumann, 1995; Westermann Rechtschreibtest: WRT 4/5 von Rathenow, 1979; WRT 6+ von Rathenow, Laupenmühlen \& Vöge, 1981; Weingartener Rechtschreibtest: WRT 1+; WRT 2+; WRT 3+ Langform; jeweils von Birkel, 1994) wurden die orthographischen Fähigkeiten erfasst. Jeder dieser Tests ist als Lückentextdiktat konzipiert. Die Rechtschreibleistung wurde mittels eines T-Werts der Gesamtnorm (nicht einer schulspezifischen Norm) beurteilt.

In der klinischen Praxis bewährten sich für die Tests Anwendungszeiträume, die zum Teil leicht von den Vorgaben der Testentwickler abweichen. Als Richtlinie für die Diagnostik der vorliegenden Untersuchung sollte das klinische Vorgehen gelten, da es auf jahrelanger Erfahrung basiert. Die genaue Festlegung der Anwendungszeiträume der jeweiligen Rechtschreibtests dokumentiert Tabelle 1. Zum Überblick listet die Tabelle auch die vom Testmanual vorgegebenen Anwendungszeiträume auf.

\begin{tabular}{|c|c|c|}
\hline Anwendungszeiträume & Tests & Normen \\
\hline Ende 1. K1. - Mitte 2. K1.: & WRT $1+$ & entspricht sich \\
\hline Mitte 2. K1. - Mitte 3. Kl.: & WRT $2+$ & Ende 2. Kl. - erste 5 Monate $3 . \mathrm{Kl}$. \\
\hline Mitte 3. Kl. - Okt. 4. Kl:: & WRT 3+ & Ende 3. Kl. - Okt. 4. Kl. \\
\hline Okt. 4. Kl. - Mitte 4. Kl:: & DRT 4 & Okt. 4. K1. - Jan. 4. K1. \\
\hline Mitte 4. Kl. - Mitte 5. Kl.: & DRT 5 & Okt. 5. Kl. - Jan. 5. Kl. \\
\hline Mitte 5. Kl. - Mitte 6. Kl:: & WRT 4 / 5 & Mitte 4. Kl. - erste 3 Monate $5 . \mathrm{Kl}$. \\
\hline ab Mitte $6 . \mathrm{Kl}$. & WRT $6+$ & Ende 5. Kl. - Anfang 7. Kl. \\
\hline \multicolumn{3}{|c|}{ „Mitte“ = Halbjahreszeugnis; KL. = Klasse } \\
\hline \multicolumn{3}{|c|}{$\begin{array}{l}\text { Tab. 1: Realisierte Anwendungszeiträume der durchgeführten Rechtschreibtests und ihre } \\
\text { Normierungsbereiche. }\end{array}$} \\
\hline
\end{tabular}


Zur Affirmation der Diagnose wurde auch die Leseleistung (Zürcher Lesetest; von Linder \& Grissemann, 1980) erhoben. Der Zürcher Lesetest erlaubt eine Einschätzung der Lesekompetenz nach vier Aspekten: Jeweils Einzelworte bzw. Leseabschnitte werden aufgrund von Fehlerzahl und Geschwindigkeit beurteilt.

Lag eine isolierte Lesestörung ohne begleitende Störung des Rechtschreibens vor, wurde der Proband von der Untersuchung ausgeschlossen.

An Versuchsmaterialien wurde außer den Testbögen nichts weiter als ein Stift benötigt; der HAWIK-III war zusätzlich durch eine Stoppuhr zu ergänzen.

\subsubsection{STICHPROBE}

\subsubsection{GESAMTSTICHPROBE}

Die Gesamtstichprobe umfasste 105 Kinder. Aufgrund des Doppelkriteriums bildeten sich in einem Koordinatensystem der Dimensionen ,Rechtschreibleistung' und ,Intelligenz' neben den beiden Experimentalgruppen (30 Kontrollprobanden, 26 lese-/rechtschreibschwache Probanden) zwei weitere, nicht auswertbare Gruppen heraus: die Gruppe der „schlechten Rechtschreiber", deren unterdurchschnittliche Rechtschreibleistung keine Diskrepanz zur Intelligenz aufwies (neun Personen) und die Gruppe der „Diskrepanten bei hohem IQ“, deren orthographische Kenntnisse zwar in Diskrepanz zu ihrer Intelligenz standen, aber ein mindestens durchschnittliches Niveau erreichten (sechs Personen).

Da bei der Untersuchung der Lese-/Rechtschreibschwäche als Lernstörung eine Konfundierung mit einer allgemeinen Lernbehinderung vermieden werden sollte, wurden Kinder mit unterdurchschnittlicher Intelligenz (IQ < 85) ebenfalls nicht in die Auswertung mit einbezogen. In diese Gruppe fielen vier Probanden.

Zwei der lese-/rechtschreibschwachen Probanden wiesen unbrauchbare Aufzeichnungen der Elektroden TP 9 und TP 10 auf, so dass für diese keine neurophysiologische Auswertung erfolgen konnte. Ihre kognitionspsychologischen Testergebnisse blieben aus Gründen der Teststärke jedoch weiterhin Bestandteil des auszuwertenden Datenmaterials.

Auf 30 weitere Probanden trafen diverse Ausschlusskriterien (siehe Punkt 4.2.5.) zu.

Abbildung 5 veranschaulicht die Zusammensetzung der Gesamtstichprobe. 


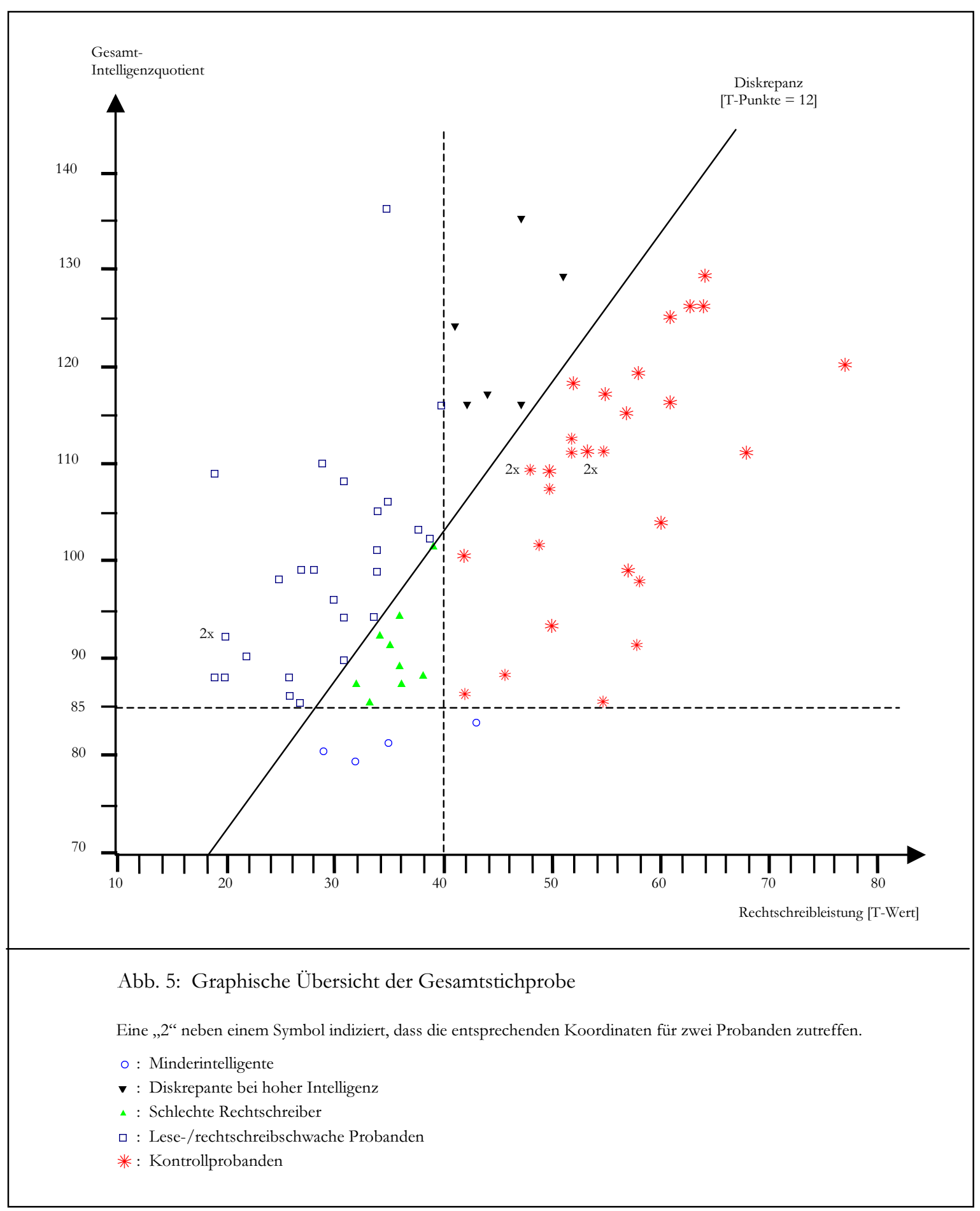




\subsubsection{PARALLELISIERUNG DER EXPERIMENTALGRUPPEN}

51 Probanden aus der Gesamtstichprobe bildeten die beiden parallelisierten Gruppen für die Auswertung. Als Parallelisierungskriterien wurden Intelligenz, das Alter und das Geschlecht berücksichtigt (siehe Punkt 4.1.). Bei der Untersuchung lese-/rechtschreibschwacher Probanden ist der Gesamt-Intelligenzquotient als Intelligenzparameter ungeeignet, da er sich auch aus zum Verbalteil gehörigen Untertestwerten zusammensetzt. Diese wiederum stellen Anforderungen, die bei lese-/rechtschreibschwachen Kindern beeinträchtigt zu sein scheinen. Die Parallelisierung erfolgte daher anhand des Handlungs- IQs.

Die Verteilung der Probanden bezüglich der Parallelisierungskriterien veranschaulicht Abbildung 6. Die Zahlenwerte der deskriptiven Statistika inklusive der Ergebnisse ihrer Signifikanztests sind der darunterstehenden Tabelle 2 zu entnehmen.

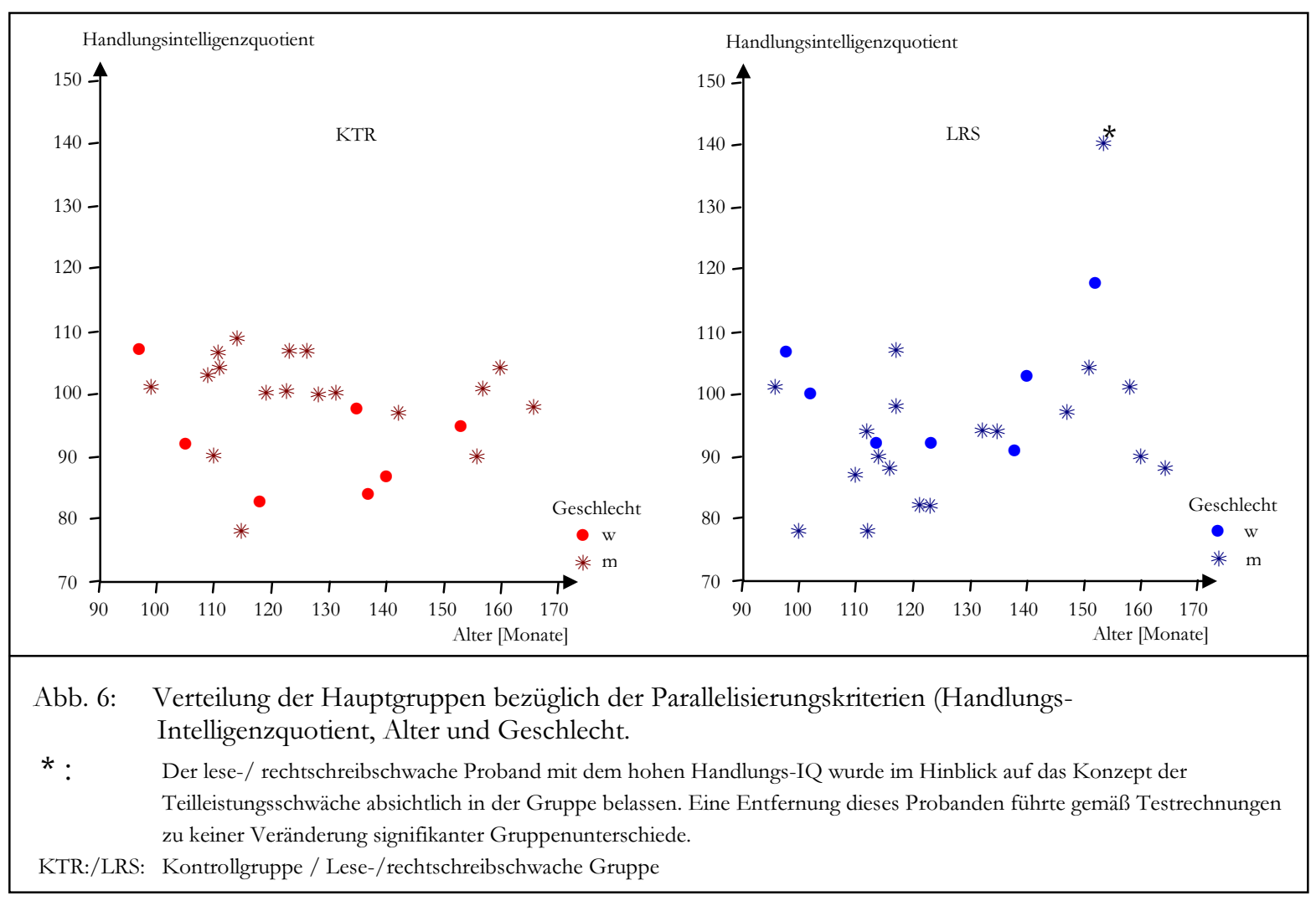




\begin{tabular}{|c|c|c|c|c|c|c|}
\hline & & KTR & LRS & df & Teststatistik & $\begin{array}{c}\mathrm{p} \\
\text { sign. }<.05\end{array}$ \\
\hline Geschlecht & $\begin{array}{l}\text { männlich } \\
\text { weiblich }\end{array}$ & $\begin{array}{c}18 \\
7\end{array}$ & $\begin{array}{c}19 \\
7\end{array}$ & 1 & $\chi^{2}=.01$ & .931 \\
\hline Alter & $\mid \begin{array}{l}\mathrm{M} / \text { Monate } 7 \\
\mathrm{~S}\end{array}$ & $\begin{array}{c}128 \\
20\end{array}$ & $\begin{array}{c}127 \\
21\end{array}$ & 1 & $\mathrm{t}=.16$ & .876 \\
\hline Handlungs - IQ & $\begin{array}{l}\mathrm{M} / \text { IO-Punkte }] \\
\mathrm{S}\end{array}$ & $\begin{array}{c}97.7 \\
8.4\end{array}$ & $\begin{array}{l}96.0 \\
13.2\end{array}$ & 1 & $\mathrm{t}=.53$ & .599 \\
\hline
\end{tabular}

Tab. 2: Deskription und Teststatistik der parallelisierten Gruppen bezüglich der Parallelisierungskriterien

Tabelle 2 und Abbildung 6 verdeutlichen die gelungene Parallelisierung der Experimentalgruppen anhand der Kriterien Geschlecht, Alter und Handlungsintelligenz. Mit etwa einem Viertel Mädchen gegenüber drei Vierteln Jungen entspricht das Geschlechterverhältnis in den Gruppen zudem in etwa dem der Population. 


\subsubsection{LESE-/RECHTSCHREIBPARAMETER DER EXPERIMENTALGRUPPEN}

Die diagnostisch relevanten kognitiven Leistungsparameter der parallelisierten Hauptgruppen sind in Abbildung 7 veranschaulicht. Die entsprechenden Mittelwerte sowie die Resultate der tTests sind in Tabelle 3 aufgeführt.

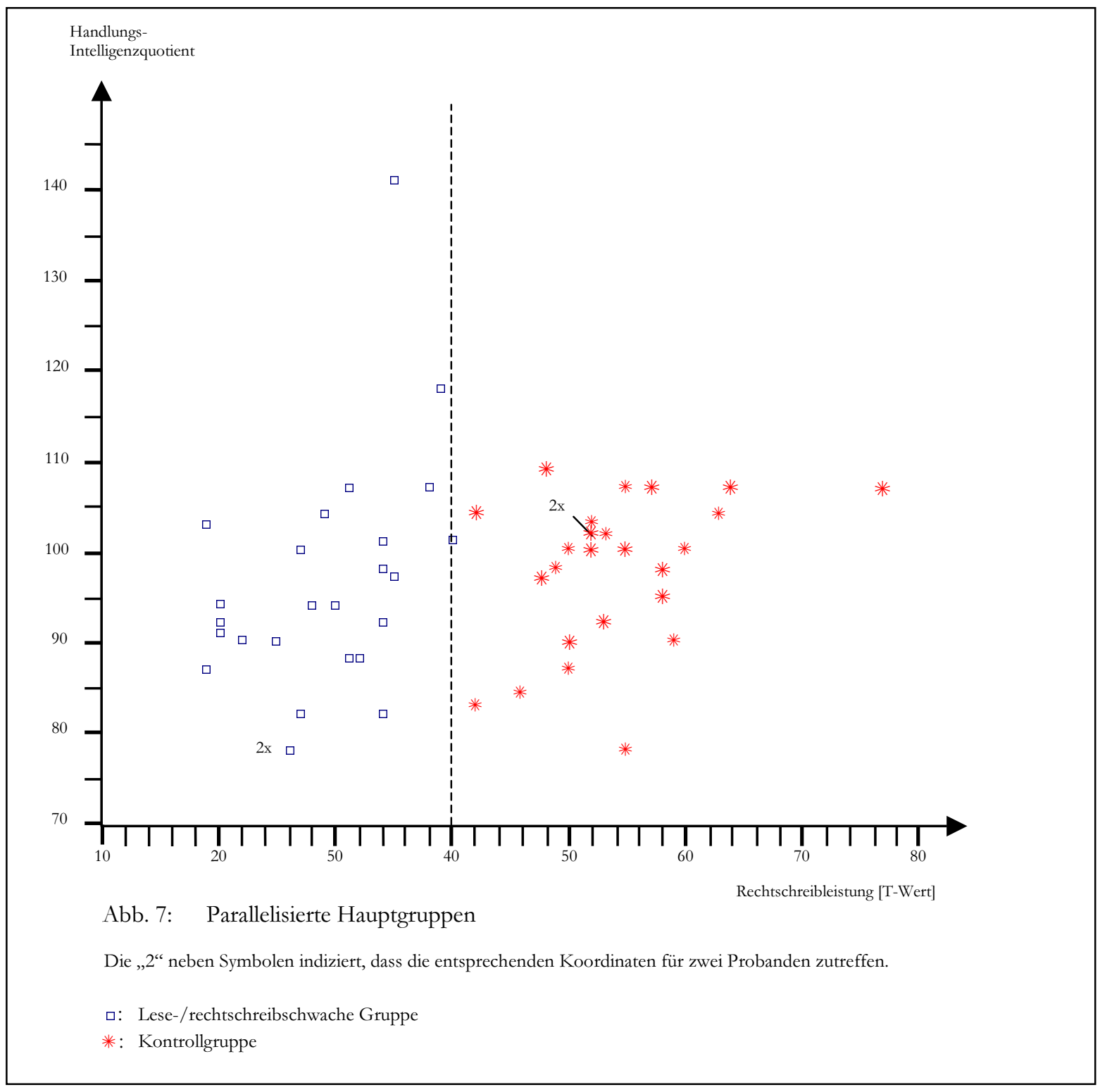




\begin{tabular}{|c|c|c|c|c|c|c|c|}
\hline & & KTR & LRS & df & $\mathbf{t}$ & $\begin{array}{c}\mathrm{p} \\
\text { sign. }<.05\end{array}$ & $\eta^{2}$ \\
\hline Rechtschreibtest: & $\begin{array}{l}\mathrm{M} \text { [T-Punkte] } \\
\mathrm{S}\end{array}$ & $\begin{array}{c}\mathbf{5 4 . 9} \\
7.4\end{array}$ & $\begin{array}{c}29.0 \\
6.4\end{array}$ & 1 & 177.8 & .000 & .784 \\
\hline $\begin{array}{l}\text { T-Differenz } \\
\text { Gesamt IQ - RS-Leistung }\end{array}$ & $\mathrm{M}$ [T-Punkte] & $\begin{array}{l}\mathbf{0 . 7} \\
7.7\end{array}$ & $\begin{array}{c}20.3 \\
6.9\end{array}$ & 1 & 106.3 & .000 & .684 \\
\hline Lesetest & & & & & $\mathbf{U}$ & $\mathrm{p}$ & \\
\hline - Einzelworte & mittlerer Rang [Zeit] & 36 & 17 & & 86 & .000 & \\
\hline - Abschnitte & mittlerer Rang [Zeit] & 35 & 17 & & 92 & .000 & \\
\hline - Einzelworte & mittlerer Rang [Fehler] & 36 & 16 & & 77 & .000 & \\
\hline - Abschnitte & mittlerer Rang [Febler] & 36 & 17 & & 81 & .000 & \\
\hline
\end{tabular}

Tab. 3: Deskription und Teststatistik der parallelisierten Gruppen bezüglich der Lese- und Rechtschreibleistung Der rote Druck der p-Werte indiziert Signifikanz-Niveaus unter 5\%.

Die Rechtschreibleistung sowie die sich daran aufspannende Diskrepanz zum Gesamt-IQ differierte zwischen den Gruppen -versuchsanlagegemäß- deutlich ( $t$ : 178; sign.: .000): Mit beinahe 55 T-Punkten (7.4) erreichten die gesunden Kinder einen Rechtschreibwert, der den der klinischen Gruppe (29; 6.4) um mehr als zwei Standardabweichungen übertraf. Dementsprechend erzeugte die Diskrepanz zum jeweils durchschnittlichen Gesamt-IQ signifikante Unterschiede (Kontrollgruppe: .7 T-Punkte; 7.7; Lese-/Rechtschreibschwache: 20.3 T-Punkte; 6.9; $t$ : 106, sign.; .000).

Die Lese-/Rechtschreibschwäche war anhand der orthographischen Leistung diagnostiziert worden. Nichtsdestotrotz sollte ein Lesetest erweisen, dass bei den untersuchten Kindern beide schriftsprachlichen Schwächen ausgeprägt sind. Der Zürcher Lesetest erlaubt eine nach vier Aspekten aufgegliederte Beurteilung der Leseleistung: Leseabschnitte und Einzelworte können jeweils bezüglich Fehlerfrequenz und Geschwindigkeit bewertet werden. Wie aus Tabelle 3 ersichtlich, produzierten die Leseanforderungen jeden Aspekts in der Tat deutliche Gruppenunterschiede im selben Sinne wie die Schreibaufgaben. 


\subsubsection{INTELLIGENZWERTE DER EXPERIMENTALGRUPPEN}

Die Ergebnisse und Teststatistiken der Untertests des HAWIK-III sowie der resultierenden Haupt-Quotienten sind in Tabelle 4 aufgelistet.

Mit 7.3 IQ-Punkten Differenz zugunsten der Kontrollgruppe (KTR: 106.3; 11.6; LRS: 99.0; 11.1; t: 5.2, sign: .02 $\rceil$ wies der Gesamt-IQ signifikante Gruppenunterschiede auf. Ursache dessen ist die Parallelisierung der Gruppen anhand des Handlungs- anstelle des Gesamt-IQs. Für eine anhand des Handlungs-IQs parallelisierte Stichprobe resultiert folglich die Erwartung einer im Verbal- und damit im Gesamtwert der Kontrollgruppe unterlegenen Leistung von seiten der lese-/rechtschreibschwachen Gruppe. Dies entspricht dem vorliegenden Fall.

Die Überlegenheit der Kontrollgruppe im Verbalteil ist tendenziell in allen zum Verbalteil gehörigen Untertests zu beobachten. In den Tests „Allgemeines Wissen“, „Gemeinsamkeiten Finden“ und „Rechnerisches Denken“ wurde diese Tendenz jedoch signifikant. Beim drittgenannten Test handelt es sich um Kopfrechnen, einer Aufgabe, die in hohem Maß den Kurzzeitgedächtnisspeicher beansprucht, welcher bei lese-/rechtschreibschwachen Probanden beeinträchtigt zu sein scheint (siehe Punkt 2.3.). „Allgemeines Wissen“ ist eine Wissensabfrage; „Gemeinsamkeiten Finden“ verlangt Abstraktionsvermögen. Schlechtere Ergebnisse bei Lese/rechtschreibschwachen lassen geringere Dichten semantischer Netzwerke vermuten. Denkbar wäre ebenfalls ein lediglich erschwerter Zugriff auf die semantische Wissensbasis. Weiterhin besteht die Möglichkeit, dass die Schwierigkeit für die Lese-/Rechtschreibschwachen in der Produktion der Antwort liegt: Alle Aufgaben des HAWIK-Verbalteils bewerten außer dem Inhalt einer Erklärung auch deren Qualität.

Im Handlungsteil halten sich (tendenzielle) Leistungsunterschiede zwischen den Gruppen in etwa die Waage. Aus welchem Grunde Kontrollkinder im „Mosaiktest“, der das Nachlegen von Mustern fordert, etwas besser ( $t: 3.1$, sign.:.086) als die Kinder der klinischen Gruppe abschnitten oder aus welchem Grund lese-/rechtschreibschwache Kinder das Ordnen einer unsortierten Bildergeschichte („Bilder Ordnen“) deutlich besser beherrschten als gesunde Kinder ( $t: 5.6$, sign.: .022), ist an dieser Stelle nicht plausibel zu erklären.

Unter den Zusatztests sticht das „Zahlen Nachsprechen“ hervor, welches einen sehr deutlichen Gruppenunterschied produzierte ( $t$ : 10.0, sign.:.003). Dieser erwartete Effekt ist auf die Auslastung der bei Lese-/Rechtschreibschwachen verminderten Kapazität des Arbeitsgedächtnisses zurückzuführen (siehe Punkt 2.3.3.3.). 


\begin{tabular}{|c|c|c|c|c|c|c|c|}
\hline \multicolumn{2}{|c|}{ Quotienten des HAWIK-III } & \multirow{2}{*}{$\begin{array}{c}\text { KTR } \\
106.3 \\
11.6 \\
\end{array}$} & \multirow{2}{*}{$\begin{array}{c}\text { LRS } \\
99.0 \\
11.1 \\
\end{array}$} & \multirow{2}{*}{$\frac{\text { df }}{1}$} & \multirow{2}{*}{$\begin{array}{c}\mathbf{t} \\
5.2\end{array}$} & \multirow{2}{*}{$\begin{array}{c}\mathrm{p} \\
\text { sign. }<.05 \\
0.027\end{array}$} & \multirow{2}{*}{$\begin{array}{c}\eta^{2} \\
0.096\end{array}$} \\
\hline Gesamt-IQ & $\begin{array}{l}\mathrm{M} \text { /IO-Punkte } 7 \\
\mathrm{~S}\end{array}$ & & & & & & \\
\hline Verbal - IQ & $\begin{array}{l}\mathrm{M} \text { /IO-Punkte] } \\
\mathrm{S}\end{array}$ & $\begin{array}{c}112.4 \\
14.0\end{array}$ & $\begin{array}{c}102.5 \\
12.3\end{array}$ & 1 & 7.2 & 0.010 & 0.129 \\
\hline Handlungs - IQ & $\begin{array}{l}\mathrm{M} \text { /IO-Punkte } 7 \\
\mathrm{~S}\end{array}$ & $\begin{array}{r}97.7 \\
8.4 \\
\end{array}$ & $\begin{array}{l}96.0 \\
13.2 \\
\end{array}$ & 1 & 0.3 & 0.599 & 0.006 \\
\hline Verbalteil & & KTR & LRS & df & t & $\mathrm{p}$ & $\eta^{2}$ \\
\hline Allgemeines Wissen & $\begin{array}{l}\mathrm{M} \text { /IO-Punkte } 7 \\
\mathrm{~S}\end{array}$ & $\begin{array}{c}11.8 \\
2.8 \\
\end{array}$ & $\begin{array}{l}9.9 \\
2.8\end{array}$ & 1 & 5.6 & 0.022 & 0.102 \\
\hline Gemeinsamkeiten Finden & $\begin{array}{l}\mathrm{M} \text { [IQ-Punkte] } \\
\mathrm{S}\end{array}$ & $\begin{array}{c}12.1 \\
2.5 \\
\end{array}$ & $\begin{array}{c}10.5 \\
2.7\end{array}$ & 1 & 5.1 & 0.028 & 0.094 \\
\hline Rechnerisches Denken & $\begin{array}{l}\mathrm{M} \text { [IQ-Punkte] } \\
\mathrm{S}\end{array}$ & $\begin{array}{c}12.3 \\
3.4 \\
\end{array}$ & $\begin{array}{l}9.9 \\
2.3 \\
\end{array}$ & 1 & 9.2 & 0.004 & 0.158 \\
\hline Wortschatz & $\begin{array}{l}\mathrm{M}[\text { IQ-Punkte }] \\
\mathrm{S}\end{array}$ & $\begin{array}{c}11.5 \\
3.3 \\
\end{array}$ & $\begin{array}{c}10.6 \\
2.7\end{array}$ & 1 & 1.2 & 0.269 & 0.025 \\
\hline Allgemeines Verständnis & $\begin{array}{l}\mathrm{M}[\text { IQ-Punkte }] \\
\mathrm{S}\end{array}$ & $\begin{array}{c}11.1 \\
2.1 \\
\end{array}$ & $\begin{array}{c}10.8 \\
2.1 \\
\end{array}$ & 1 & 0.4 & 0.549 & 0.007 \\
\hline Handlungsteil & & KTR & LRS & df & t & $\mathrm{p}$ & $\eta^{2}$ \\
\hline Bilder Ergänzen & $\begin{array}{l}\mathrm{M} \text { /IO-Punkte] } \\
\mathrm{S}\end{array}$ & $\begin{array}{l}9.9 \\
2.5 \\
\end{array}$ & $\begin{array}{l}9.8 \\
2.8 \\
\end{array}$ & 1 & 0.0 & 0.922 & 0.000 \\
\hline Zahlen-Symbol-Test & $\begin{array}{l}\mathrm{M} \text { [IQ-Punkte] } \\
\mathrm{S}\end{array}$ & $\begin{array}{l}9.6 \\
2.5 \\
\end{array}$ & $\begin{array}{l}8.4 \\
3.1 \\
\end{array}$ & 1 & 2.4 & 0.129 & 0.046 \\
\hline Bilder Ordnen & $\begin{array}{l}\mathrm{M}[\text { IQ-Punkte }] \\
\mathrm{S}\end{array}$ & $\begin{array}{l}9.5 \\
2.3 \\
\end{array}$ & $\begin{array}{c}11.1 \\
2.5 \\
\end{array}$ & 1 & 5.6 & 0.022 & 0.103 \\
\hline Mosaik-Test & $\begin{array}{l}\mathrm{M} \text { [IQ-Punkte }] \\
\mathrm{S}\end{array}$ & $\begin{array}{c}10.5 \\
2.7 \\
\end{array}$ & $\begin{array}{l}8.9 \\
3.7 \\
\end{array}$ & 1 & 3.1 & 0.086 & 0.059 \\
\hline Figurenlegen & $\begin{array}{l}\mathrm{M} / \text { IQ-Punkte }] \\
\mathrm{S}\end{array}$ & $\begin{array}{l}8.9 \\
2.5 \\
\end{array}$ & $\begin{array}{l}8.7 \\
3.1 \\
\end{array}$ & 1 & 0.1 & 0.810 & 0.001 \\
\hline Zusatztests & & KTR & LRS & df & $\mathrm{t}$ & $\mathrm{p}$ & $\eta^{2}$ \\
\hline Symboltest & $\begin{array}{l}\mathrm{M} \text { /IO-Punkte }\rceil \\
\mathrm{S}\end{array}$ & $\begin{array}{l}9.6 \\
2.4 \\
\end{array}$ & $\begin{array}{l}9.3 \\
2.4 \\
\end{array}$ & 1 & 0.3 & 0.582 & 0.006 \\
\hline Zahlen Nachsprechen & $\begin{array}{l}\mathrm{M} / \text { IQ-Punkte }] \\
\mathrm{S}\end{array}$ & $\begin{array}{c}10.2 \\
2.5 \\
\end{array}$ & $\begin{array}{l}8.1 \\
2.2 \\
\end{array}$ & 1 & 10.0 & 0.003 & 0.169 \\
\hline Labyrinth & $\begin{array}{l}\mathrm{M} \text { [IQ-Punkte }] \\
\mathrm{S}\end{array}$ & $\begin{array}{c}11.8 \\
2.7\end{array}$ & $\begin{array}{c}10.4 \\
3.4\end{array}$ & 1 & 2.8 & 0.102 & 0.056 \\
\hline
\end{tabular}

Tab. 4: Mittelwerte und Ergebnisse der t-Tests der Untertests sowie der resultierenden Quotienten des HAWIK-III.

Ein roter und fetter Druck eines p-Wertes kennzeichnet einen auf dem 5\% - Niveau, ein blauer und fetter Druck einen auf dem 10\%-Niveau signifikanten Gruppenunterschied. 


\subsubsection{AUSSCHLUSSKRITERIEN}

Wie bereits erwähnt, wurden Kinder mit einem Gesamtintelligenzquotienten unter 85 von der Auswertung ausgeschlossen. Bilingual oder nicht deutschsprachig aufgewachsene Kinder nahmen an der Untersuchung ebenfalls nicht teil. Selbiges traf für den Fall zu, dass ein Tinnitus, eine Hörschwellenasymmetrie, Schwerhörigkeit oder eine Erkrankung an Mittelohrentzündung innerhalb einer Woche vor der Untersuchung vorlag.

Psychiatrische Komorbiditäten wurden akzeptiert, sofern sie keine ursächliche Verbindung zur Lese-/Rechtschreibschwäche aufwiesen oder mögliche Begleitsymptome der Lese-/Rechtschreibstörung wie Anpassungsstörungen, depressive Störungen, Angst oder Störung des Sozialverhaltens sein könnten. Verhaltensauffälligkeiten, emotionale Beeinträchtigungen oder außergewöhnliche psychosoziale Umstände, auf die eine vorliegende Lese-/Rechtschreibschwäche jedoch hätte zurückgeführt werden können, bedingten einen Ausschluss aus der Studie. Ebenso wurden keine Probanden untersucht, bei denen Psychosen, tiefgreifende Entwicklungsstörungen, Tic-Störungen oder Zwangsstörungen diagnostiziert wurden. Da es Hinweise darauf gibt, dass die Art der basalen Funktionsdefizite bei komorbider hyperkinetischer Störung sowohl quantitativ als auch qualitativ von den Funktionsdefiziten reiner lese/rechtschreibschwacher Probanden abweicht (Banaschewski, Tiffin-Richards, Hasselhorn, Rothenberger, 2000), wurden für diese Studie auch Kinder mit zusätzlicher ADHD ausgeschlossen.

\subsubsection{INSTRUMENTE ZUR ERFASSUNG POTENTIELLER AUSSCHLUSS-} KRITERIEN

Um eine möglichst saubere störungsbezogene Zusammensetzung der Experimentalgruppen zu erreichen, wurde der Erhebung des psychopathologischen Status viel Aufmerksamkeit beigemessen. Aus diesem Grunde wurden ein gewisses Maß an Überschneidungen der durch die verschiedenen Instrumente erhobenen Information in Kauf genommen.

Mit einem Elternteil wurde das strukturierte diagnostische Interview KIDDIE-SADS (Schedule for Affective Disorder in Schizophrenia for School Age Children; Orvarschel \& Puig-Antich, 1987) durchgeführt. Desweiteren hatten Eltern und Lehrer jeweils einen anamnestischen Fragebogen zum Verhalten von Kindern und Jugendlichen der Achenbach-Reihe (CBCL Child Behavior Checklist; Achenbach, 1991; TRF - Teacher Report Form, Achenbach, 1991). Die Achenbach-Skalen indizieren Auffälligkeiten auf verschiedenen psychopathologischen Dimensionen (Sozialer Rückzug, somatische Beschwerden, Angst/Depression, Selbstun- 
sicherheit, psychotische Tendenzen, Aufmerksamkeit, Delinquenz, Aggressives Verhalten). Diese Instrumente verschafften einen Überblick über die psychopathologische Befindlichkeit.

Für die Prüfung einer möglichen Aufmerksamkeits- oder Hyperaktivitätsstörung wurden zwei zusätzliche Instrumente zu Rate gezogen. Es handelte sich dabei um die 10-Items-Version des Conners-Fragebogen (Goyette, Conners \& Ulrich, 1978), den Eltern und Lehrer beantworten mussten, und den Fremdbeurteilungsbogen für HKS (FBB-HKS; Döpfner \& Lehmkuhl, 1998). Beim Conners-Fragebogen gilt als auffällig, wer eine Itemwert-Summe von 14 überschreitet. Die Döpfner-Liste enthält die 18 diagnostischen Items aus DSM IV bzw. ICD 10, die sich zur Hälfte auf eine Aufmerksamkeits- und zur anderen Hälfte auf eine Hyperaktivitäts-/Impulsivitätsskala beziehen. Gemäß des diagnostischen Standards mussten sechs Items einer der beiden Skalen deutlich bejaht (d.h. auf einer der beiden höheren Werte auf einer vierstufigen Skala angekreuzt) worden sein, um ein Kind als auffällig einzustufen.

Aus der Gesamtheit der Informationen aus dem Interview und den verschiedenen Fragebögen wurde schließlich das klinische Urteil gebildet und auf dessen Grundlage über einen möglichen Ausschluss aufgrund eines Vorliegens komorbider Störungen entschieden.

Die Hörfähigkeit wurde vor der Untersuchung anhand einer Audiometrie geprüft: Die Hörschwelle keines Ohres sollte im Sprachbereich (500 bis $4000 \mathrm{~Hz}$ ) über $30 \mathrm{~dB}$ liegen. Eine eventuelle Hörschwellenasymmetrie durfte $10 \mathrm{~dB}$ nicht überschreiten.

\subsubsection{PSYCHOPATHOLOGISCHER STATUS DER EXPERIMENTALGRUPPEN}

Ein Überblick über die psychopathologische Befindlichkeit der beiden selegierten Experimentalgruppen soll mithilfe einer Tabelle (Tab. 5) der Ergebnisse von CBCL und TRF gegeben werden: Wie es für eine von einer Störung betroffenen Gruppe zu erwarten ist, betrug die Abweichung von der Norm (50) bei der schriftsprachlich gestörten Gruppe auf jeder Skala mehr als bei der Kontrollgruppe. Dabei bewegten sich die Normabweichungen durchaus im akzeptablen Rahmen, da keine den kritischen Wert von einer Standardabweichung (10 TPunkte) überschritt. 


\begin{tabular}{|c|c|c|c|c|c|c|c|c|c|c|c|c|}
\hline & & \multicolumn{3}{|c|}{ internalisierende Störungen } & \multirow[b]{2}{*}{$\begin{array}{l}\text { IV. } \\
\text { social } \\
\text { prob- } \\
\text { lems }\end{array}$} & \multirow[b]{2}{*}{$\begin{array}{c}\text { V. } \\
\text { thought } \\
\text { prob- } \\
\text { lems }\end{array}$} & \multicolumn{3}{|c|}{ externalisierende Störungen } & \multicolumn{3}{|c|}{ zusammenfassende Werte } \\
\hline & & $\begin{array}{c}\text { I. } \\
\text { with- } \\
\text { drawn }\end{array}$ & $\begin{array}{c}\text { II. } \\
\text { somatic } \\
\text { com- } \\
\text { plaint }\end{array}$ & $\begin{array}{l}\text { III. } \\
\text { anxious. } \\
\text { depres- } \\
\text { sion }\end{array}$ & & & $\begin{array}{l}\text { VI. } \\
\text { atten- } \\
\text { tion } \\
\text { prob- } \\
\text { lems }\end{array}$ & $\begin{array}{l}\text { VII. } \\
\text { delin- } \\
\text { quent } \\
\text { beha- } \\
\text { vior }\end{array}$ & $\begin{array}{l}\text { VIII. } \\
\text { aggres- } \\
\text { sive } \\
\text { beha- } \\
\text { vior }\end{array}$ & Gesamt & $\begin{array}{l}\text { inter- } \\
\text { nalisie- } \\
\text { rende } \\
\text { Störun- } \\
\text { gen }\end{array}$ & $\begin{array}{l}\text { exter- } \\
\text { nalisie- } \\
\text { rende } \\
\text { Störun- } \\
\text { gen }\end{array}$ \\
\hline CBCL & $\mathrm{M}$ & 52.7 & 54.4 & 52.7 & 50.7 & 50.7 & 51.6 & 52.1 & 51.1 & 42.3 & 45.5 & 43.3 \\
\hline KTR & $\mathrm{s}$ & 4.7 & 5.2 & 4.7 & 2.3 & 2.2 & 4.0 & 4.3 & 3.6 & 9.9 & 10.6 & 9.4 \\
\hline CBCL & M & 56.0 & 58.5 & 55.4 & 53.6 & 55.0 & 56.5 & 54.0 & 53.6 & 51.4 & 53.4 & 48.0 \\
\hline LRS & $\mathrm{s}$ & 6.7 & 7.8 & 6.1 & 5.6 & 6.5 & 7.4 & 6.1 & 6.4 & 12.7 & 11.4 & 11.7 \\
\hline TRF & $\mathrm{M}$ & 52.0 & 50.6 & 51.7 & 51.8 & 50.0 & 50.6 & 51.0 & 51.9 & 44.2 & 46.4 & 47.4 \\
\hline KTR & $\mathrm{s}$ & 4.0 & 1.9 & 2.4 & 3.7 & 0.0 & 2.8 & 2.3 & 3.3 & 6.6 & 6.6 & 6.6 \\
\hline TRF & $\mathrm{M}$ & 57.7 & 56.2 & 58.3 & 57.3 & 51.6 & 57.5 & 54.3 & 54.6 & 55.4 & 57.1 & 51.1 \\
\hline LRS & $\mathrm{s}$ & 8.0 & 8.4 & 8.2 & 6.8 & 4.4 & 7.4 & 5.9 & 7.3 & 9.8 & 11.8 & 10.0 \\
\hline
\end{tabular}

Tab. 5: $\quad$ Ergebnisse von CBCL und TRF: Angabe jeweils in T-Werten.

KTR: Kontrollgruppe / LRS: lese-/rechtschreibschwache Gruppe

Ein besonderer Fokus war auf den Ausschluss der häufig komorbiden Aufmerksamkeitsdefizit /Hyperaktivitätsstörung gelegt worden. Die in der Experimentalgruppe vorhandenen durchschnittlichen Tendenzen hinsichtlich Aufmerksamkeitsdefiziten bzw. Hyperaktivität/Impulsivität lassen sich der Itemwertsumme der Conners-Fragebögen bzw. der Anzahl deutlich bejahter DSM IV - Items entnehmen (vgl. Tab. 6). Trotz des Ausschlusses von einer ADHD betroffener Kinder lagen die Werte der schriftsprachlich gestörten Kinder stets (etwas) über den Werten der Kontrollprobanden. Der Abstand zu den jeweils kritischen Werten blieb jedoch ausreichend hoch, um davon ausgehen zu können, dass keine bedeutsamen Tendenzen hinsichtlich einer ADHD vorlagen.

\begin{tabular}{|c|c|c|c|c|c|}
\hline & & $\begin{array}{c}\text { Conners Eltern } \\
\text { [Itemwertsumme] }\end{array}$ & $\begin{array}{c}\text { Conners Lehrer } \\
\text { [Itemwertsumme] }\end{array}$ & $\begin{array}{c}\text { Skala Hyper- } \\
\text { aktivität/Impulsivität } \\
\text { [Anzahl Items] }\end{array}$ & $\begin{array}{l}\text { Skala Aufmerksamkeit } \\
\text { [Anzahl Items] }\end{array}$ \\
\hline KTR & $\begin{array}{c}\mathrm{M} \\
\mathrm{S}\end{array}$ & $\begin{array}{l}2.5 \\
2.5\end{array}$ & $\begin{array}{l}2.5 \\
3.2\end{array}$ & $\begin{array}{l}.1 \\
.3 \\
\end{array}$ & $\begin{array}{l}.3 \\
.7 \\
\end{array}$ \\
\hline LRS & $\begin{array}{c}\mathrm{M} \\
\mathrm{S}\end{array}$ & $\begin{array}{l}7.0 \\
4.5\end{array}$ & $\begin{array}{l}7.0 \\
6.2\end{array}$ & $\begin{array}{l}1.2 \\
1.9\end{array}$ & $\begin{array}{l}2.5 \\
2.7\end{array}$ \\
\hline
\end{tabular}

Tab. 6: $\quad$ Ergebnisse der Eltern- und Lehrer-Conners-Fragebögen (kritischer Wert: $\geq 15$ ) und der DSM IVSkalen (kritischer Wert: $\geq 6$ ).

KTR: Kontrollgruppe / LRS: Lese-/rechtschreibschwache Gruppe 


\subsection{ABHÄNGIGE VARIABLEN}

Den Probanden wurden Aufgaben aus den Bereichen der phonologischen Bewusstheit, dem Arbeitsgedächtnis inklusive seiner Fähigkeit des Zugriffs auf das Langzeitgedächtnis und dem auditiven sensorischen Gedächtnis gestellt.

\subsubsection{PHONOLOGISCHE VERARBEITUNG}

\subsubsection{TESTS ZUR PHONOLOGISCHEN BEWUSSTHEIT}

Die phonologische Bewusstheit wurde zum einen durch vier Untertests einer von Marx (1998) entwickelten Testbatterie zur Erfassung der phonologischen Bewusstheit in der Grundschule („Würzburger Testbatterie“) geprüft. Zwei weitere Aufgaben stammten aus dem Psycholinguistischen Entwicklungstest (PET) von Angermaier (1974). Die Würzburger Batterie umfasste die Tests „Vokale Ersetzen“, die „Restwort-Aufgabe“, „Phonemvertauschung“ und „Lautkategorisierung“. Die Tests des PET waren das „Laute Verbinden“ und das „Worte Ergänzen“. Beim „Vokale Ersetzen“ wurde das Ersetzen eines jeden /a/ in einem vorgesprochenen Wort durch ein /i/ verlangt. Aus „Ananas“ wurde demzufolge „Ininis“. Es wurden insgesamt 15 Worte vorgegeben. Als korrekt gewertet wurde eine Antwort, wenn nicht nur die Vokale richtig substituiert, sondern auch die an die substituierten Vokale angrenzenden Laute richtig wiedergegeben worden waren.

Das „Laute Vertauschen" verlangte die Vertauschung der ersten beiden Laute eines vorgesprochenen Wortes. „Radio“ wurde folglich zu „ardio“. Hierbei war für eine Wertung als ,korrekt` nur die gelungene Umstellung der ersten beiden Buchstaben vonnöten; doch wurde diese Information dem Kind vorenthalten, um den Schwierigkeitsgrad der Aufgabe aufrecht zu erhalten. Die Aufgabe umfasste 15 Worte; bei den fünf letzten handelte es sich um Pseudoworte.

Bei der „Restwort-Aufgabe“ war die Fähigkeit zur Phonemanalyse gefragt. Das Kind musste das erste Phonem eines vorgesprochenen Wortes entfernen und das Restwort nennen. Dieses musste vollständig korrekt wiedergegeben worden sein. Wiederum setzte sich diese Aufgabe aus 15 Worten zusammen, von denen die letzten fünf Pseudoworte waren.

Im letzten Untertest der Batterie, dem „Laute-Kategorisieren“ ging es um das Zuordnen von Anlauten bzw. Endlauten. Die Versuchsleiterin sprach vier einsilbige Worte bzw. vier sinnlose 
Silben vor. Drei Items der Sequenz begannen oder endeten jeweils mit dem gleichen Phonem. Das Kind musste das Wort identifizieren, dessen Anlaut bzw. dessen Endlaut sich von dem der anderen drei unterschied. Ein Beispiel für die Kategorisierung der Anlaute wäre die Itemreihe /mib/ moch/ /mog/ /nog/; ein Beispiel für eine Sequenz zur Endlaut-Kategorisierung stellen die Items /zoss/ /ress/ /poss/ /reff/ dar. In beiden Fällen ist das letzte Item die richtige Lösung. Es wurden sechs Anlaut- und sechs Endlaut-Sequenzen dargeboten.

Beim „Laute Verbinden“ des PET wurden dem Probanden 33 in Teile zergliederte Worte präsentiert. Die Aufgabe des Kindes bestand darin, das Wort als Ganzes zu erkennen und zu benennen. Mit fortschreitender Aufgabe wurden die Einheiten, in die ein Wort aufgespalten wurde, immer kleiner, bis schließlich nur noch einzelne Phoneme dargeboten wurden. Weiterhin fügten sich die Einzellaute der letzten Items nicht mehr zu realen, sondern zu Pseudowörtern zusammen. Dieser Test prüfte demzufolge die Fähigkeit zur Phonemsynthese.

Die 36 Worte des „Worte Ergänzens“ waren durch Buchstabenauslassungen verfremdet. Je weiter die Aufgabe voranschritt, desto mehr Auslassungen enthielt ein Wort. Das Kind musste die Worte erkennen und benennen.

Die Präsentation der Aufgaben des PET erfolgte anhand einer CD.

Wie aus den Aufgabenbeschreibungen deutlich geworden ist, verlangten die Untertests „Vokale Ersetzen“, die „Restwort-Aufgabe“ und das „Laute Vertauschen“ sprachliche Operationen auf der Phonemebene und lassen sich demzufolge der ,phonologischen Bewusstheit im engeren Sinne“ zuordnen. Beim „Laute Kategorisieren“ und den Aufgaben des PET bestanden die zu manipulierenden Spracheinheiten (zumeist) aus mehreren Phonemen und fallen daher in die Kategorie der ,phonologischen Bewusstheit im weiteren Sinne‘.

Zur Bestimmung der Leistung eines Kindes wurden bei jedem Test der phonologischen Bewusstheit die korrekt beantworteten Items summiert.

Für die Durchführung der Tests war außer einem Protokollbogen, auf welchem die Tests zusammengestellt waren (siehe Anhang A), lediglich ein CD-Spieler nötig, da die Items einiger Tests (PET-Untertests) auf CD vorhanden waren. 


\subsubsection{TESTS ZUM ARBEITSGEDÄCHTNIS}

Die funktionelle Gesamtkapazität des Arbeitsgedächtnisses wurde mit dem „Zahlen Nachsprechen“"und der „Wortspanne“(Hasselhorn, 1986) geprüft. Bei diesen Aufgaben wurde eine Sequenz einstelliger Zahlen bzw. einsilbiger Worte mit gleichmäßigem Rhythmus (jeweils ein Item pro Sekunde) vorgesprochen. Bei der Wortspanne konnten die Itemfolgen von einer Kassette abgespielt werden. Der Proband musste die Abfolge unmittelbar nach der Präsentation vollständig und in der richtigen Reihenfolge wiedergeben. Pro Sequenzlänge wurden jeweils zwei Sets vorgegeben. Wurden beide Item-Sequenzen einer Spannenlänge korrekt reproduziert, wurde die entsprechende Wortspanne attestiert; für jede nicht gekonnte Sequenz wurde von dem maximal erreichten Niveau ein halber Punkt abgezogen. Einem Probanden, der beispielsweise die beiden Zwei-Item-Sequenzen korrekt beantwortet, von der Drei-ItemSequenz sowie von der Vier-Item-Sequenz nur eine und schließlich keine mehr richtig löst, würde demzufolge eine Wortspanne von 3 bescheinigt werden.

Nach insgesamt zwei nicht korrekt reproduzierten Sequenzen wurde die Wortspanne abgebrochen. Beim „Zahlen Nachsprechen“ des HAWIK-III wurde als zusätzliche Besonderheit verlangt, die Itemsequenz in umgekehrter Reihenfolge wiederzugeben. Beide Varianten des "Zahlen Nachsprechens" waren nach zwei aufeinanderfolgenden falschen Antworten abzubrechen.

Zur Kapazitätsbestimmung des phonetischen Speichers wurde der auf Grete Mottier (1951) zurückgehende Tests des Kunstwörter-Nachsprechens („Mottier-Test ‘) eingesetzt. Die 30 Kunstworte wurden per CD dargeboten und waren vom Probanden unmittelbar nach der Präsentation zu wiederholen. Durch die Steigerung der Wortlänge von zwei bis auf sechs Silben wurde die Aufgabenschwierigkeit moduliert. Die korrekt wiedergegebenen Worte wurden aufsummiert.

Mithilfe des Kunstwort-Nachsprechens sollte neben dem Speichervolumen auch die Verarbeitungspräzision des phonetischen Speichers ermittelt werden. Die insgesamt 36 Items wurden von CD abgespielt. Das Itemmaterial variierte entlang der beiden Dimensionen „Silbenanzahl“ und „Verzerrungsgrad“: Zwei-, drei- oder viersilbige Pseudoworte wurden unverzerrt, verzerrt oder stark verzerrt dargeboten.

Zur Schätzung der Rehearsal-Geschwindigkeit wurde die Artikulationsgeschwindigkeit bestimmt. Die Probanden mussten das Worttripel („Trigramme“) „Baum - Schuh - Topf“ möglichst schnell zehn Mal hintereinander aufsagen (vgl. Hasselhorn, Lingelbach \& Gabert, 
1991). Dabei sollten sie diejenige Geschwindigkeit anschlagen, die es ihnen gerade noch erlaubte, das Tripel fehlerfrei und in wohlartikulierter Weise wiederzugeben. Die Sprechsequenz wurde mittels eines Diktiergeräts auf Kassette aufgenommen. Die präzise Bestimmung der Sprechdauer erfolgte nach einer Transformation des Sprechmaterials in digitales Format (.wav-Datei) mithilfe des Klangverarbeitungsprogramms „Cool Edit 96“.

Mit Ausnahme des "Zahlen Nachsprechens" als Bestandteil des HAWIK-IIIs war die Arbeitsgrundlage der Tests zum Arbeitsgedächtnis der bereits erwähnte Protokollbogen (siehe Anhang A). An weiteren Materialien waren eine Stoppuhr, ein Kassettenrekorder, die Kassette mit den Mottier-Items, ein CD-Spieler für die CD mit den Kunstworten, ein Diktiergerät (Marke Sony) inklusive Leerkassette und die Software „Cool Edit 96“ notwendig.

\subsubsection{TESTS ZUM ABRUF AUS DEM LANGZEITGEDÄCHTNIS}

Zur Ermittlung der Fähigkeit, auf die semantische Wissensbasis zuzugreifen, wurden zwei Tests durchgeführt: Es handelte sich hierbei um einen Wortproduktionstest, den „Test 5“ des Horn'schen Prüfsystem für Schul- und Bildungsberatung (1969), und um das „Farbstriche Benennen“ des Farbe-Wort-Interferenztests („FWIT“) (,STROOP-Test“) von Bäumler (1985).“

Die Aufgabe beim Wortproduktionstest bestand darin, zu gegebenen Anfangsbuchstaben binnen jeweils einer Minute möglichst viele Worte zu generieren. Dieser Test lädt gemäß Horn deutlich auf Thurstones Faktor der Wortflüssigkeit.

Zusätzlich zu den Buchstaben „L“ und „P“ wurde in dieser Untersuchung „R“ verlangt. Die Versuchsleiterin schrieb die produzierten Worte mit und bildete sowohl die Gesamtsumme als auch die Summe pro Buchstabe generierter Worte. Wortdopplungen oder fremdsprachige Worte wurden dabei nicht berücksichtigt. Das mehrfache Nutzen desselben Stammes wie Wortumformungen („Lehrer“ / „Lehrerin“) oder Wortkombinationen („Postbote“ / „Postauto") wurden eher als strategische Leistungen denn als das Resultat von Suchprozessen beurteilt und dementsprechend ebenfalls nicht gewertet.

Das Rohdatum des „Farbstriche Benennens“ bestand in der Benennungsgeschwindigkeit der Farben 72 gelber, blauer, grüner oder roter Balken der ersten Farbbalkentafel des FWIT. 
Für die Durchführung der Tests zum Abruf aus dem Langzeitgedächtnis wurden folglich nebst Stift und Protokollbogen eine Stoppuhr und die erste Tafel zum „Farbstriche Benennen“ des FWIT benötigt.

\subsubsection{AUDITIV SENSORISCHES GEDÄCHTNIS}

\subsubsection{KONZEPTION EINES PARADIGMAS}

Für die Untersuchung auditiv sensorischer Verarbeitungsdefizite kann das Mismatch Negativity - Paradigma eingesetzt werden. Im Gegensatz zu den kognitionspsychologischen Konstrukten stehen hierbei jedoch keine standardisierten Testverfahren bereit. Sie sind demzufolge auf der Basis in der Literatur berichteter Befunde und auf der Basis im Vorfeld der Untersuchung getroffener Erwartungen zu entwickeln.

Da, wie aus Punkt 3.3. hervorgeht, derartige auditiv sensorische Verarbeitungsdefizite am wahrscheinlichsten gehalten werden, die in den Kontext der drei konkurrierenden Hypothesen (rasch temporales Defizit, spezifisch sprachliches Defizit, generell auditives Defizit) einzuordnen sind, ist ein MMN-Paradigma entlang dieser drei Hypothesen zu konzipieren. Dieser Aufgabe widmet sich der folgende Abschnitt.

Das Mismatch Negativity - Paradigma bedarf stets eines Stimuluspaares von Standard und Deviant. Der Kontrast zwischen diesen beiden beinhaltet zweierlei Aspekte: die Art der Stimuli zum einen und die Art des Reizunterschiedes zum anderen.

Für die Überprüfung eines spezifisch sprachlichen Defizits sind mit Sicherheit sprachliche Stimuli, die einen sprachlichen Kontrast konstituieren, geeignet. Vor dem Hintergrund der obigen Überlegung stellt sich jedoch die Frage, ob nicht ein einfacherer Kontrast, beispielsweise ein Tonhöhenkontrast, ebenfalls defizitär verarbeitet wird, wenn er durch spektral komplexe Sprachstimuli getragen wird. Eine derartige Differenzierung zwischen diesen beiden Aspekten hatten Schulte-Körne et al. (1998c, 1999b) in ihrer Studie nicht vorgenommen, in welcher sie aus Lese-/Rechtschreibschwäche-bedingten Gruppenunterschieden bei einem sprachlichen Kontrast, nicht aber bei einem Frequenzkontrast zwischen einfachen Sinustönen eine Sprachspezifizität des Verarbeitungsdefizits ableiteten. Die Überprüfung eines spezifisch sprachlichen Defizits verlangt demnach auch den Zwischenschritt eines nicht-phonologischen Kontrastes auf der Basis phonologischer Stimuli. 
Optimale Kandidaten für die Wahl der Sprachstimuli sind diejenigen, die immer wieder in engen Zusammenhang mit Sprachverarbeitungsdefiziten gebracht wurden bzw. bei Diskriminationsversuchen sich wiederholt als die am schwierigsten $\mathrm{zu}$ detektierenden Kontraste erwiesen haben, nämlich Konsonant-Vokal-Silben, die mit einem Stop-Konsonanten beginnen (Csépe \& Gyurkócza, 1995; Mody, 1993; Mody et al., 1995; Schulte-Körne et al., 1998c, 1999b). Letztere wiederum unterscheiden sich hinsichtlich zweier Merkmale, der Stimmhaftigkeit und dem Artikulationsort. Für das Aufzäumen phonologische Kontraste eignen sich demzufolge Konsonant-Vokal-Silben, deren Konsonant ein Verschlusslaut ist und zwischen Standard und Deviant bezüglich Stimmhaftigkeit oder Artikulationsort variiert.

Bei Mody et al. (1995) war der Gruppenunterschied bei der Detektion des KonsonantenKontrastes erst durch die Beschleunigung der Darbietung entstanden. Da aber Schulte-Körne et al. (1998c; 1999b) auch ohne eine Geschwindigkeitserhöhung signifikante Ergebnisse erzielt hatten und eine Anforderung mit gesteigerten Ansprüchen an die rasche temporale Verarbeitung ohnehin als gesonderte Aufgabe gestellt wird, soll an dieser Stelle darauf verzichtet werden. Vorteil dieser Vorgehensweise ist die Vermeidung einer Konfundierung der beiden Kriterien „Sprachlichkeit“ und „rasche temporale Verarbeitung“.

Zusammenfassend kann hiermit folgendes festgehalten werden: Für die Überprüfung des spezifisch sprachlichen Defizits werden sprachliche Standard-Stimuli gewählt. Zwei phonologische Kontraste werden durch Deviants gebildet, die sich in Stimmhaftigkeit oder Artikulationsort vom Standard unterscheiden. Ein nichtphonologischer Kontrast kommt durch eine Frequenzveränderung des phonologischen Standards zustande.

Ein generelles auditorisches Defizit müsste bei der Verarbeitung auch der physikalisch unkomplexesten Kontraste und Stimuli zum Tragen kommen. Zu diesen zählen beispielsweise Frequenzkontraste von Sinustönen; ein Paradigma, das sich seit Entwicklung der Methode als sehr zuverlässig in der Auslösung von Mismatch Negativites erwiesen hat (Näätänen et al., 1978). Obgleich nicht immer (Schulte-Körne et al. 1998c; 1999b), wurden anhand dieses Paradigmas auch Lese-/Rechtschreibschwäche-bedingte Gruppenunterschiede deutlich (z.B. Baldeweg, Richardson, Watkins, Foale, \& Gruzelier, 1999). Aus diesen Gründen bietet es sich an, zur Prüfung der Hypothese eines generellen auditorischen Verarbeitungsdefizits lese-/rechtschreibschwacher Personen einen Frequenzkontrast von Sinustönen in die Untersuchung einzubeziehen.

Die dritte zu prüfende Hypothese bildet die des Defizits rasch temporaler Verarbeitung bei lese/rechtschreibschwachen Personen. Gemäß einer Kernaussage dieser These liegen den 
Schwierigkeiten Lese-/rechtschreibschwacher Schwierigkeiten bei der Auflösung rascher auditiver Veränderungen zugrunde, welche ein grundlegendes Charakteristikum von Sprache und insbesondere von Stop-Konsonanten- bilden. Kontraste zur Prüfung dieser Theorie müssen demzufolge schnelle Veränderungen enthalten. Bestmöglich würden diese Veränderungen sowohl an komplexen Sprachstimuli als auch an völlig unkomplexen Reizen realisiert, um zu differenzieren, ob die Verarbeitungsschwierigkeit auf einen erschwerten Umgang mit Sprachstimuli selbst oder auf die (zusätzliche) Verarbeitung einer raschen Veränderung zurückzuführen ist. Die benötigten Paradigmata könnten somit auf der Basis der bereits entwickelten nichtphonologischen sowie phonologischen Stimuli gebildet werden. Für beide Typen Stimuli wurden Frequenzkontraste vorgeschlagen. Hielten die Deviants die Transposition jeweils nur für kurze Zeit, so entstünden Kontraste, die die Fähigkeit zur Verarbeitung rascher Veränderungen erfassen. Zur Prüfung der These des Defizits rascher temporaler Verarbeitung bieten sich demzufolge Reizpaare an, die durch eine jeweils nur kurz währende Frequenzveränderung des nichtphonologischen bzw. des phonologischen Standardstimulus gebildet wurden.

Zum Überblick kann festgehalten werden, dass zur Untersuchung der Art eines möglichen Verarbeitungsdefizits bei Lese-/Rechtschreibschwäche phonologische und nicht-phonologische Stimuli mit phonologischen und nicht-phonologischen Kontrasten verschieden kombiniert werden. Es entstehen dabei folgende Kombinationen:

- Phonologische Kontraste auf der Basis phonologischer Stimuli (,phonologische Bedingungen“)

Stimuli: Konsonant-Vokal-Silben

Kontrastierungskriterien: Artikulationsort; Stimmbaftigkeit

- Nicht-phonologische Kontraste auf der Basis phonologischer Stimuli („nicht-phonologische Bedingungen")

Stimuli: Konsonant-Vokal-Silben

Kontrastierungskriterien: Frequenæ; kursfristige Frequenæänderung

- Nicht-phonologische Kontraste auf der Basis nicht-phonologischer Stimuli („Sinus-Bedingungen“)

Stimuli: Sinustöne

Kontrastierungskriterien: Frequenæ; kurafristige Frequen₹änderung 


\subsubsection{HERSTELLUNG DER MMN-STIMULI}

Für das entworfene MMN-Paradigma wurden zwei Standardstimuli und sechs Deviants benötigt. Deren Herstellung und physikalische Parameter werden in diesem Abschnitt beschrieben. Die Stimuli wurden, um eine möglichst vollständige und originale spektrale Komplexität zu gewährleisten, auf der Grundlage gesprochener Silben gewonnen. Ihre Bearbeitung erfolgte digital mithilfe der Software „Cool Edit 96“.

Als Standard für die phonologische Bedingung wurde die Konsonant-Vokal-Silbe /ba/ gewählt. Hinsichtlich Artikulationsort unterscheidet sich die Silbe /ga/ (,phonologischer Deviant /ga/“, dPH-G), hinsichtlich der Stimmhaftigkeit die Silbe /pa/ (,phonologischer Deviant /pa/“, dPH-P). Diese beiden konstituierten demzufolge die phonologischen Deviants der vorliegenden Studie.

Um alle phonologischen Stimuli auf dieselbe Tonlage $\mathrm{zu}$ bringen, mussten die /a/s der Deviants durch das/a/ des Standards ersetzt werden. Die diskriminationskritischen Konsonanten dieser Silben blieben dabei unverändert. Da die Gesamtdauer der Reize konstant gehalten werden sollte (286 ms), die Dauer von Konsonanten jedoch differiert, unterschieden sich die Längen der anzufügenden /a/s jeweils minimal.

Aufstellungen der physikalischen Kriterien (Formantenverläufe, Bandbreiten) des phonologischen Standards und seiner phonologischen Deviants sind in den Tabellen 7 und 8 zu finden: 


\begin{tabular}{|c|c|c|c|c|}
\hline & \multicolumn{2}{|l|}{ „B“ } & \multicolumn{2}{|l|}{ „A“ } \\
\hline & $\begin{array}{l}\text { Extremum der For- } \\
\text { mantentransition bei } \\
\text { lageverändernden For- } \\
\text { manten* bzw. mittlere } \\
\text { Formantenfrequenz } \\
\text { zwischen } 0 \text { und } 19 \mathrm{~ms} \\
\text { bei lagekonstanten } \\
\text { Formanten } * * \\
{[\mathrm{~Hz}]}\end{array}$ & $\begin{array}{l}\text { Bandbreite } \\
{[\mathrm{Hz}]}\end{array}$ & $\begin{array}{l}\text { Mittlere } \\
\text { Formantenfrequenz } \\
\text { zwischen } 20 \text { und } \\
286 \mathrm{~ms} \\
{[\mathrm{~Hz}]}\end{array}$ & $\begin{array}{l}\text { Bandbreite } \\
{[\mathrm{Hz}]}\end{array}$ \\
\hline Grundfrequenz & 200 & & 200 & \\
\hline Formant 1 (F 1) & $315(13 \mathrm{~ms})^{*}$ & 150 & 780 & 180 \\
\hline Formant 2 (F 2) & $1000(5,5 \mathrm{~ms})^{*}$ & 130 & 1400 & 180 \\
\hline Formant 3 (F 3) & $2540(5 \mathrm{~ms})^{*}$ & 120 & 3070 & 280 \\
\hline Formant 4 (F 4) & $3850(0-19 \mathrm{~ms})^{* *}$ & 210 & 4070 & 300 \\
\hline Formant 5 (F 5) & $5190(0-19 \mathrm{~ms})^{* *}$ & 270 & 5170 & 250 \\
\hline
\end{tabular}

Frequenz [Hz]

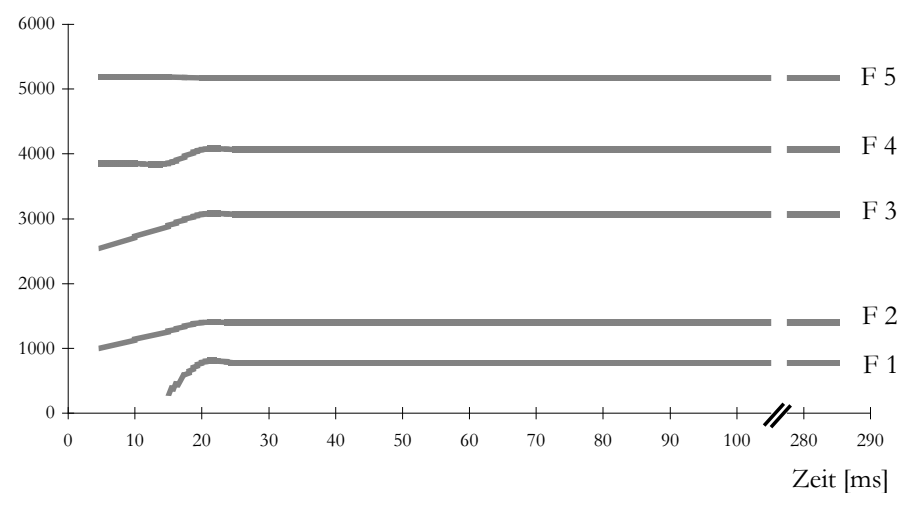

Tab. 7: Formantenverläufe des phonologischen Standards in tabellarischer Form und als schematische Darstellung. Die Bandbreiten wurden in der Abbildung nicht nachempfunden. 


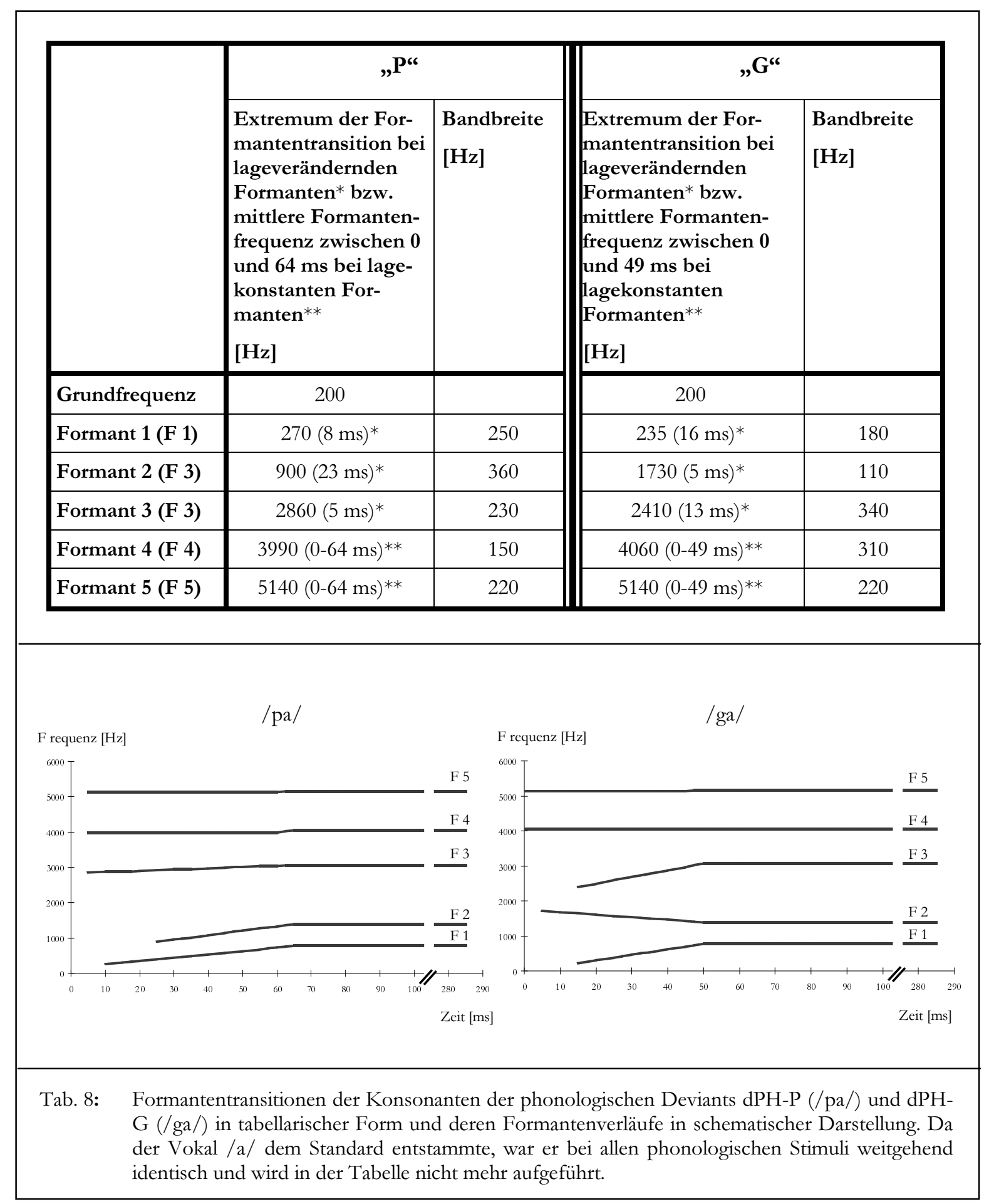


Die nicht-phonologischen Deviants des phonologischen Standards wurden durch Transposition des Standardstimulus gebildet. Das Maß der Frequenzerhöhung wurde mittels einer PilotVersuchsreihe so bestimmt, dass die Entdeckungsschwierigkeit des transponierten Stimulus in einem aktiven Diskriminationsparadigma -gemessen über Entdeckungswahrscheinlichkeit und Reaktionsgeschwindigkeit- der Entdeckungswahrscheinlichkeit der phonologischen Deviants entsprach. Dieser kritische Wert lag bei einer Transposition um 0.9 ganze Töne.

Während einer der nicht-phonologischen Deviants die Diskriminationsfähigkeit einer einfachen Frequenzänderung eines phonologischen Stimulus prüfen sollte, sollte ein anderer zusätzliche Anforderungen an die Verarbeitung sich rasch ändernder Information stellen. Ein nichtphonologischer Deviant wurde folglich durch Transposition des Standards über seine gesamte Länge gebildet (,statischer nicht-phonologischer Deviant“, dNP-S); bei einem zweiten wurde die Transposition so bald wie möglich wieder zurückgenommen (,dynamischer nichtphonologischer Deviant“, dNP-D). Dieser Zeitpunkt betrug 120 ms. Im Verlauf der folgenden 40 ms fielen die erhöhten Frequenzen wieder auf ihr Ursprungsniveau zurück, wo sie bis zum Ende des Stimulus blieben.

Die Formantenverläufe der eben beschriebenen nicht-phonologischen Stimuli sind in Tabelle 9 wiedergegeben. 


\begin{tabular}{|c|c|c|c|}
\hline & „B“ & „A“ & „A“ \\
\hline & $\begin{array}{l}\text { Extremum der Formantentransition } \\
\text { bei lageverändernden Formanten* } \\
\text { bzw. mittlere Formantenfrequenz } \\
\text { zwischen } 0 \text { und } 19 \text { ms bei lage- } \\
\text { konstanten Formanten** } \\
{[\mathrm{Hz}]}\end{array}$ & $\begin{array}{l}\text { Mittlere For- } \\
\text { mantenfrequenz } \\
\text { ab } 20 \mathrm{~ms} \\
\text { NP-S: } 20-286 \mathrm{~ms} \\
\text { NP-D: } 20-120 \mathrm{~ms} \\
{[\mathrm{~Hz}]}\end{array}$ & $\begin{array}{l}\text { Mittlere For- } \\
\text { mantenfrequenz } \\
\text { des NP-D von } 160 \\
\text { bis } 286 \text { ms } \\
{[\mathrm{Hz}]}\end{array}$ \\
\hline Grundfrequenz & 220 & 220 & 200 \\
\hline Formant 1 (F 1) & $380(12 \mathrm{~ms})^{*}$ & 850 & 790 \\
\hline Formant 2 (F 2) & $1050(5 \mathrm{~ms})^{*}$ & 1470 & 1410 \\
\hline Formant 3 (F 3) & $2690(5 \mathrm{~ms})^{*}$ & 3280 & 3090 \\
\hline Formant 4 (F 4) & $4270(0-19 \mathrm{~ms})^{* *}$ & 4390 & 4110 \\
\hline Formant 5 (F 5) & $5450(0-19 \mathrm{~ms})^{* *}$ & 5440 & 5210 \\
\hline
\end{tabular}

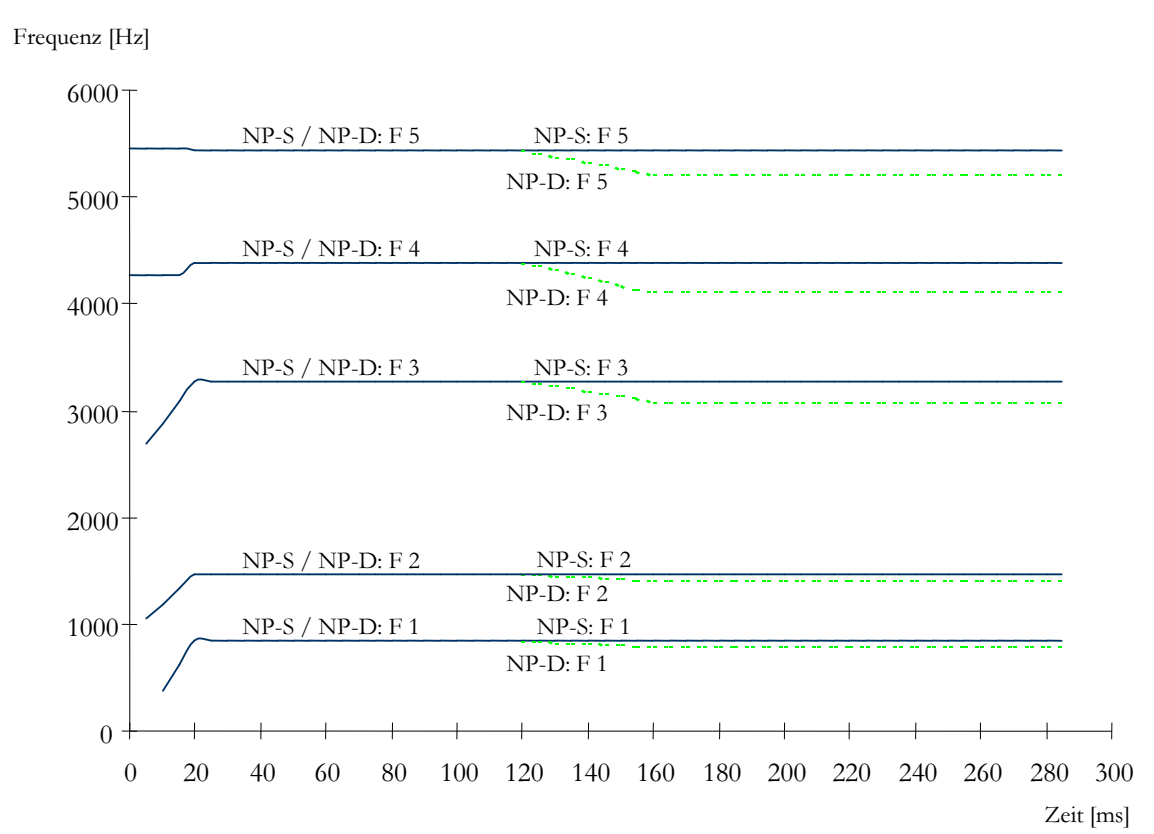

Tab. 9: Formantenverläufe des statischen nicht-phonologischen Deviants (dNP-S) und des dynamischen nicht-phonologischen Deviants (dNP-D) in tabellarischer Form und in schematischer Darstellung. Der dNP-S wurde durch Transposition des phonologischen Standards über seiner gesamten Länge erzeugt. Beim dNP-D bestand die Transposition nur über die ersten $120 \mathrm{~ms}$; nach einem $40 \mathrm{~ms}$ langem Übergang wurde die Frequenzlage des Standards wieder erreicht. In der Abbildung beschreibt die blaue durchgezogene Linie den Verlauf des dNP-S und bis zum Zeitpunkt von 120 ms ebenso den Verlauf des bis dorthin identischen dNP-D; den weiteren, abweichenden Verlauf des dNP-D stellt die grüne gestrichelte Linie dar. 
Der nicht-phonologische Standardstimulus sollte sehr unkomplexer Natur sein; es wurde folglich ein Sinuston einer Frequenz von $1000 \mathrm{~Hz}$ konstruiert, der dieselbe Gesamtlänge wie die übrigen Reize besaß. Als Frequenzkontrast diente ein $100 \mathrm{~Hz}$ höherer Sinuston (,statischer Sinuston-Deviant“; dSin-S). Da die Verarbeitung sich rasch ändernder Information auch für einfache Grundreize geprüft werden sollte, wurde in einem weiteren Deviant die Frequenzerhöhung nach $120 \mathrm{~ms}$ während einer Phase von insgesamt $40 \mathrm{~ms}$ wieder zurückgenommen, so dass der Stimulus ab dem Zeitpunkt von $160 \mathrm{~ms}$ die Frequenz von $1000 \mathrm{~Hz}$ erreicht hat (,dynamischer Sinuston-Deviant“; dSin-D). Die Frequenzverläufe der Sinus-Stimuli veranschaulicht Abbildung 8.

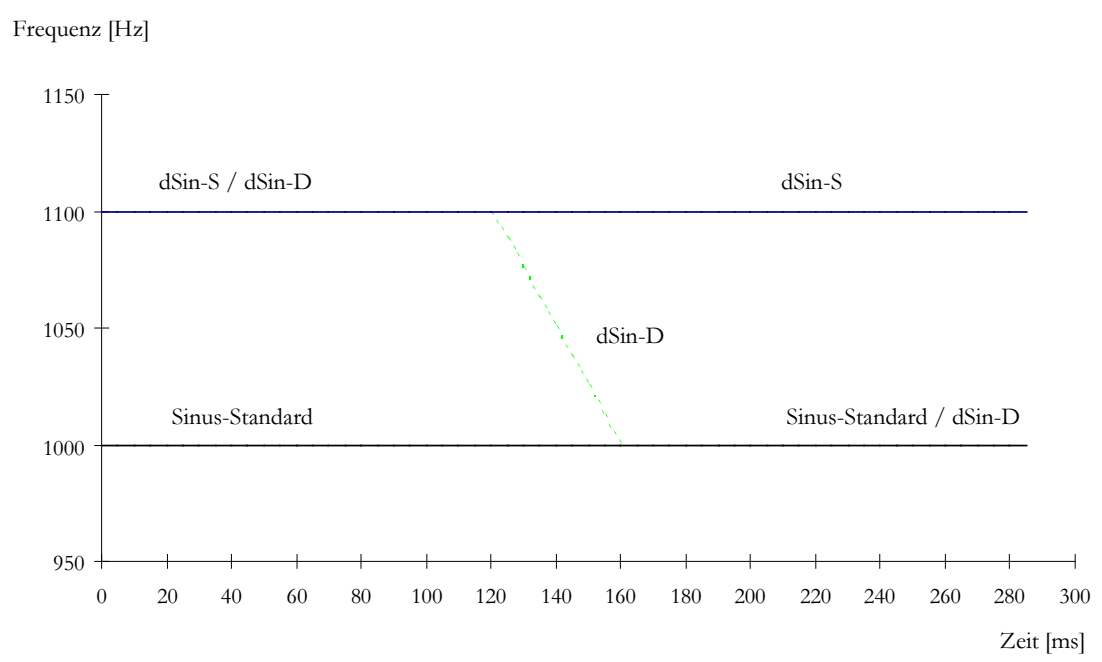

Abb. 8: Frequenzverläufe des Sinus-Standards und der Sinus-Deviants (statischer Sinus-Deviant dSin-S, dynamischer Sinus-Deviant dSin-D) in schematischer Darstellung. Der dSin-S wurde durch Transposition des Sinus-Standards über seiner gesamten Länge erzeugt. Beim dSin-D bestand die Transposition nur über die ersten $120 \mathrm{~ms}$; nach einem $40 \mathrm{~ms}$ langem Übergang wurde die Frequenzlage des Standards wieder erreicht. In der Abbildung beschreibt die schwarze durchgezogene Linie den Verlauf des Standards und die blaue durchgezogene Linie den Verlauf des dSin-S. Der dSin-D war bis zum Zeitpunkt von $120 \mathrm{~ms}$ mit dem Verlauf des dSin-S und ab dem Zeitpunkt von $160 \mathrm{~ms}$ mit dem Verlauf des Standards identisch. Den Übergang markiert die gestrichelte grüne Linie. 


\subsubsection{APPLIKATION DES MMN-PARADIGMAS}

Die Darbietung des MMN-Paradigmas erfolgte computergesteuert. Routinen, die im Programm „Neuroscan“ (STIM, SCAN; Compumedics) erstellt worden waren, organisierten den Ablauf im Rahmen eines Oddball-Paradigmas.

Aus den Kombinationen der in diesem Abschnitt beschriebenen Stimuli resultiert jeweils pro Deviant eine Mismatch Negativity. Die Namen für die Bedingungen wurden daher aus den Namen der Deviants generiert (Weglassung des Buchstabens „d“ zu Anfang des Kürzels).

Ein Ableitungsabschnitt fasste jeweils zwei Bedingungen zusammen: Es wurden dabei die beiden phonologischen Deviant-Bedingungen (PH-P, PH-G), die beiden nicht-phonologischen Deviant-Bedingungen aus dem phonologischen Stimulus (NP-S, NP-D) und die beiden SinusBedingungen (SIN-S, SIN-D) kombiniert. Jeder Abschnitt enthielt 2000 pseudorandomisierte Stimuli (1600 Standards plus jeweils 200 der beiden Deviants). Durchschnittlich vier und mindestens drei Standards trennten die Deviants voneinander. Zur Vermeidung von Erwartungseffekten (Bildung einer $\mathrm{CNV}$ ) wurde das Interstimulusintervall variabel gewählt (600 +/- max. 100 ms). Die Tonreize wurden über einen Kopfhörer (Marke: Sennheiser) binaural mit einer mittleren Lautstärke von $75 \mathrm{~dB}$ SPL dargeboten, während die Versuchspersonen zum Binden der Aufmerksamkeit einen kindgerechten tonlosen Videofilm verfolgten.

Zur Ableitung wurden die Ag/AgCl-Elektroden (Fa. Nihon Kohden; Elektrodencreme: Marke Grass EC2) gemäß des internationalen 10/20-Systems (vgl. Abb. 9) auf der Kopfhautoberfläche

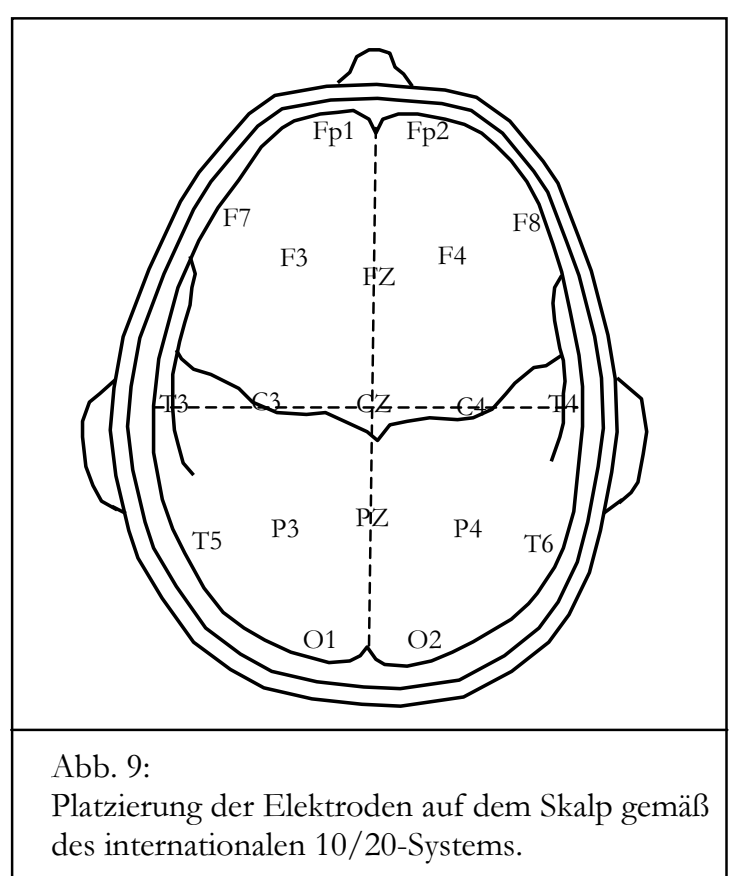
des Kindes platziert (Fp1, Fp2, F7, F8, F3, F4, $\mathrm{Fz}, \mathrm{C} 3, \mathrm{C} 4, \mathrm{Cz}, \mathrm{T} 3, \mathrm{~T} 4, \mathrm{~T} 5, \mathrm{~T} 6, \mathrm{P} 3, \mathrm{P} 4, \mathrm{Pz}, \mathrm{O} 1$, $\mathrm{O} 2$; die Nasenelektrode diente als Ableitungsreferenz, da sie v.a. bei der Identifikation (topographische Abgrenzung gegen N2b) hilfreich ist; die Erdungselektrode liegt bei Fpz). Vertikal angebrachte VEOG-Elektroden dienten der Kontrolle von Augenbewegungen und Blinks.

Die Impedanzen sollten unter $5 \mathrm{k} \Omega$ bleiben. Das EEG wurde mit einem Synamps-Verstärker verstärkt und kontinuierlich mit einer oberen Grenzfrequenz von $0,1 \mathrm{~Hz}$ und einer samplingrate von $250 \mathrm{~Hz}$ aufgezeichnet. 


\subsubsection{BEARBEITUNG DES KONTINUIERLICHEN EEGS}

Bevor die Extraktion der eigentlich interessierenden Rohvariablen in einer EEG-Untersuchung möglich ist, muss die kontinuierliche Aufzeichnung gefiltert, korrigiert, epochiert und gemittelt werden. Diese Arbeitsgänge erfolgten mit dem Programm „Brain Vision Analyzer, Version 1.03“ (Brain Products, München).

In einem ersten Schritt durchliefen die Daten einen Hochpass- (70 Hz, 24 dB/okt.), einen Tiefpass- $(0.1 \mathrm{~Hz}, 24 \mathrm{~dB} / \mathrm{okt}$.) und gegen die Netzspannung einen Notchfilter $(50 \mathrm{~Hz})$. Nach einer Umreferenzierung des EEG auf die Elektroden TP9 und TP10 wurden die Signale in Abschnitte von 800 ms Länge plus einer Prästimulus-Epoche von 100 ms segmentiert. Anhand der VEOGs wurden die Kanäle aller Elektroden um Augenartefakte bereinigt. Der Korrekturalgorithmus entsprach dem Ansatz von Gratton, Coles \& Donchin (1983). Gemäß diesem wurden solche Epochen verworfen, in denen absolute Spannungen von mehr als +/- $200 \mu \mathrm{V}$ auftraten, in denen die Spannungsspanne innerhalb dieser Absolutwerte $200 \mu \mathrm{V}$ überschritt oder in denen Spannungssprünge von einem zum folgenden Messzeitpunkt einen Betrag von $70 \mu \mathrm{V}$ übertrafen.

Anhand der Prästimulus-Epoche wurde eine baseline-Korrektur vorgenommen, d.h. die während der $100 \mathrm{~ms}$ vor Reizbeginn registrierte Signalamplitude auf durchschnittlich $0 \mu \mathrm{V}$ gesetzt. Anschließend konnte der probandenspezifische Epochenmittelwert eines jeden MMNTyps gebildet werden (Average). Ein Average, zu welchem weniger als 20 Reizdurchgänge beigetragen hätten, wäre vom folgenden Rechenschritt, der Bildung des Grand Averages, d.h. des gruppenspezifischen mittleren Kurvenverlaufs, ausgeschlossen worden. Das gesetzte Minimum von 20 Reizdurchgängen war jedoch in allen Fällen erreicht worden.

\subsubsection{AUSWAHL DER ELEKTRODE(N)}

Im folgenden Schritt galt es, eine Elektrode bzw. einen Zusammenschluss von Elektroden auszuwählen, dessen Daten die MMN am deutlichsten zeigen würden. Nachdem die Mismatch Negativity als ein frontozentral auftretendes Potenzial definiert ist, kamen hierfür nur die Elektroden Fz, Cz, C3, C4, F3 und F4 in Frage. Topographische Abbildungen der Aktivität über den Zeitverlauf zeigten, dass die intensivste negative Aktivität in Reaktion auf Stimuli konsistent bei der Elektrode Fz auftrat. Diese wurde demzufolge als Lieferantin der Mismatch Negativity - Daten ausgewählt. 


\subsubsection{SEgMENTIERUNG DER Mismatch NEGATIVITY - KURVEN}

Die resultierenden Grand Averages der Elektrode Fz wiesen in den meisten Fällen zwei, in einem Falle sogar drei Gipfel auf. Es lag folglich nahe, die einzelnen MMN-Antworten in Teilabschnitte zu segmentieren. Ebenso mussten Anfang und Ende einer MMN-Kurve definiert werden. Bei der Festlegung der Segmentgrenzen wurden die -in der Regel sehr ähnlichenVerläufe beider Experimentalgruppen berücksichtigt, so dass jeder diskriminative Zeitpunkt den besten Kompromiss zwischen den jeweiligen Talsohlenzeitpunkten der MMNs beider Gruppen darstellte.

Die Global Field Power, ein Maß der elektrischen Gesamtspannung zu einem gegebenen Zeitpunkt, diente bei der Definition der Segmentgrenzen ebenfalls als Orientierung.

\subsubsection{EXTRAKTION DER MisMATCH NEGATIVITY - VARIABLEN}

Ein hirnelektrisches Potenzial bedarf zweier Hauptkriterien zu seiner hinreichenden Beschreibung, nämlich der Auftretenslatenz sowie der Reaktionsintensität. Beide Parameter sollten infolgedessen zur Beschreibung einer Mismatch Negativity erhoben werden.

Als Maß für die Reaktionslatenz der Mismatch Negativity wurde die Zeitspanne vom StimulusOnset bis zum maximalen peak erhoben. Die Reaktionsintensität sollte mittels der Fläche unter der Kurve erfasst werden. Als alternativer Intensitätsindikator könnte die Amplitude des maximalen peaks herangezogen werden. Um jedoch die Gefahr einer Überbewertung eventuell zufälliger hoher Ausschläge einzuschränken, wurde die Fläche als ein Maß der Gesamtintensität ausgewählt. Damit resultierte für jedes Segment eine Latenz- und eine Flächenvariable. 


\subsection{KONZEPTE ZUR UNTERSUCHUNG EINES ZUSAMMEN- HANGS ZWISCHEN KOGNITIONSPSYCHOLOGISCHEN UND AUDITIV SENSORISCHEN VERARBEITUNGS- DEFIZITEN}

Das Hauptziel der vorliegenden Studie besteht darin, einen möglichen Zusammenhang zwischen kognitionspsychologischen und neurophysiologischen Verarbeitungsdefiziten bei lese/rechtschreibschwachen Kindern festzustellen und gegebenenfalls $\mathrm{zu}$ spezifizieren. Diese Aufgabe beruht auf einem erfolgreichen Nachvollziehen von Verarbeitungsdefiziten, wie es in den vorangegangenen Abschnitten 4.2. und 4.3. bzw. 3.1. und 3.2. beschrieben wurde. Die Exploration eines Zusammenhangs bedarf keiner weiterer Tests oder Untersuchungen; die erforderlichen Konzepte und Variablen müssen mithilfe aufbauender rechnerischer Schritte generiert werden. Rechnerische Operationen gelten nicht im engeren Sinn als „Methode“ und mögen daher in Methodenkapitel zunächst fehlplatziert erscheinen. Da die entsprechenden Auswertungstechniken aber erst $\mathrm{zu}$ den Konzepten führen, die die Beantwortung der Fragestellung schließlich ermöglichen, wie es auch aus Tests stammende Rohvariablen tun, soll die Auswertungsstrategie dennoch an dieser Stelle skizziert werden.

Berechnungen wurden mithilfe des Programms SPSS 10 (Statistical Package for the Social Sciences, Version 10) erstellt.

Das Aufdecken und Definieren von Gemeinsamkeiten zwischen kognitionspsychologischen und neurophysiologischen Verarbeitungsschwierigkeiten gliedert sich in zwei Abschnitte. Im ersten Abschnitt stehen gegenseitige Abhängigkeiten der Konstrukte bzw. Verarbeitungsebenen sowie auch die Frage nach unabhängigen Bereichen im Mittelpunkt. In einem zweiten Abschnitt soll der Zusammenhang modelliert werden.

Um Abhängigkeiten zwischen Konstrukten und Ebenen herauszuarbeiten, müssen diese Konstrukte und Ebenen klar und sicher definiert sein. Um dies sicherzustellen, wurde in einem ersten Schritt für jedes Konstrukt (phonologische Bewusstheit, phonologisches Arbeitsgedächtnis, Abruf aus dem Langzeitgedächtnis) und für die beiden Ebenen (kognitionspsychologische Ebene, neurophysiologische Ebene) eine Variablenaggregation ausgewählt, die gleichzeitig möglichst sparsam (mit einer Minimalzahl an Variablen und inhaltlichen Redundanzen) und möglichst vollständig ein Konstrukt / eine Ebene repräsentierte.

Die gegenseitige Abhängigkeit der Konstrukte und Ebenen ist auf deren gegenseitige Abhängigkeit in der jeweiligen Relation zur Lese-/Rechtschreibschwäche bezogen. Solche Abhängigkeiten lassen sich als Erklärungspotenziale (Varianzen) bei der Vorhersage einer Zielvariablen 
operationalisieren. Dabei erschien es allerdings unter dem Aspekt der Vielfalt statistischer Möglichkeiten sinnvoll, als Explanandum nicht die nominalverteilte Gruppenvariable, sondern die intervallskalierte Rechtschreibleistung einzusetzen. Aus theoretischer Sicht war dies vertretbar, da die störungsspezifischen Schwierigkeiten in der schriftsprachlichen Leistung liegen; das Hinzuziehen des Intelligenzfaktors dient lediglich dem Aufspannen einer Diskrepanz, deren Sinnhaftigkeit zudem von Zeit zu Zeit in Zweifel gezogen wird (Klicpera \& Gasteiger-Klicpera, 2001; Marx, Weber \& Schneider, 2001; siehe auch Punkt 4.2.2.) und somit ein politischen Einflüssen unterworfenes Kriterium darstellt. Da die Probandenselektion bezüglich der Rechtschreibleistung kontinuierlich erfolgte, war dieses Vorgehen auch unter methodischem Aspekt zu rechtfertigen.

Für die Berechnungen im Rahmen des ersten Schritts bei der Definition eines Zusammenhangs zwischen kognitionspsychologischen und neurophysiologischen Verarbeitungsdefiziten bei lese/rechtschreibschwachen Kindern standen somit für ein Konstrukt / eine Ebene optimal repräsentative Variablenaggregate zur Verfügung, anhand derer die Erklärungspotenziale zur Vorhersage der Rechtschreibleistung eines jeden Konstrukts / jeder Ebene bestimmt werden konnten und mithilfe derer Gemeinsamkeiten der Konstrukte / Ebenen bei der Vorhersage der Rechtschreibleistung ermittelt werden konnten.

Da die Konstrukte / Ebenen in einer inhaltlich aufeinander aufbauenden Rangfolge zueinander stehend vermutet werden, sollte der ermittelte Zusammenhang in ein Modell gefasst werden. Für diese Art von Fragestellung eignet sich eine kausalanalytische Herangehensweise. Die in 3.3. formulierte Modellerwartung soll demzufolge in die Aufstellung eines LISREL-Modells münden, wobei die im Schritt zuvor definierten Variablenaggregate als latente Variablen dienen sollten.

\subsection{VERSUCHSABLAUF}

Vor der Untersuchung wurden die Eltern der Versuchspersonen mithilfe eines Informationsblattes und gegebenenfalls auch mündlich über Ziele und Ablauf der Studie aufgeklärt. Ihr Einverständnis zu der Teilnahme ihres Kindes mussten sie auf einem entsprechenden Formular dokumentieren. Im Zuge des Aufklärungsgespräches wurden auch die Fragebögen (CBCL, TRF, Conners-Elternversion, Conners-Lehrerversion) ausgegeben. Falls die Probanden Patienten der Poliklinik waren, wurden Information und Fragebogenausgabe zum Teil von den behandelnden Therapeuten übernommen. 
Als Aufwandsanerkennung für die Teilnahme an der Studie erhielten die Probanden 25 Euro und evtl. weitere 10 Euro für Fahrtkosten. Eine Rückmeldung über die Leistungen des Kindes ging an die behandelnden Therapeuten, sofern die Kinder aus der Poliklinik rekrutiert worden waren; die Familien anderweitig geworbener Kinder (v.a. Kontrollkinder) erhielten per Brief eine Rückmeldung zu den Leistungen ihres Kindes.

Der Untersuchung ging weiterhin eine audiometrische Prüfung der Hörfähigkeit voraus.

Die Untersuchung verteilte sich auf drei Termine, die zur Gewährleistung eines adäquaten Aufmerksamkeitsniveaus jeweils nicht nach 16.00 Uhr begonnen wurden. Für jeden Termin waren etwa zwei Stunden einzuplanen. Während eines dieser Termine wurde mit einem Elternteil das diagnostische Interview (Kiddie-SADS) durchgeführt und der FBB-HKS ausgefüllt. Die Untersuchungen fanden in ruhigen Laborräumen statt.

Das Untersuchungsprogramm war auf die drei Termine wie folgt aufgeteilt, wobei die Reihenfolge der Termine variiert wurde:

- Intelligenztest (HAWIK-III)

- Kognitionspsychologische Tests, Lesetest, Rechtschreibtest

- $\quad$ EEG-Ableitung

Die Durchführung des HAWIK-III folgte den Vorgaben des Manuals. Alle Untertests wurden durchgeführt. Die Tests zur phonologischen Bewusstheit, zum Arbeitsgedächtnis, zum Zugriff auf das Langzeitgedächtnis sowie der Lese- und der Rechtschreibtest fanden an einem weiteren Termin statt. Die Reihenfolge der Tests entsprach derjenigen auf dem Protokollbogen (siehe Anhang A). Für den Lese- und den Rechtschreibtest wurden die altersentsprechenden Durchführungsanweisungen befolgt. Die Ergebnisse aller Tests wurden in ein Datenblatt (siehe Anhang B) eingetragen, welches die Grundlage für die Übertragung in das Datenverarbeitungsprogramm (SPSS 10) bildete.

Vor Beginn der EEG-Ableitung wurde das Kind aufgefordert, sich aus einer vorhandenen Auswahl kindgerechter Videofilme einen Film auszusuchen, den es während der Ableitung stumm verfolgte. Die Ableitung jeden Abschnittes nahm in etwa 25 Minuten in Anspruch; dazwischen wurden kleine Pausen gemacht. 


\subsection{EMPIRISCHE VORHERSAGEN}

\subsubsection{KOGNITIONSPSYCHOLOGISCHE VERARBEITUNGSDEFIZITE}

Gemäß der psychologischen Hypothese 1.0 bestehen bei lese-/rechtschreibschwachen Probanden Schwierigkeiten der phonologischen Verarbeitung. Die Hypothese integriert drei untergeordnete Hypothesen, laut derer die Verarbeitung im phonologischen Arbeitsgedächtnis $(\mathrm{pH}$ 1.a), die Leistung in der phonologischen Bewusstheit ( $\mathrm{pH}$ 1.b) und die Leistung beim Abruf aus dem Langzeitgedächtnis ( $\mathrm{pH}$ 1.1) beeinträchtigt ist.

Aus der psychologischen Hypothese 1.a wird die empirische Vorhersage eV 1.a abgeleitet:

Die Kinder der lese-/rechtschreibschwachen Gruppe zeigen im Vergleich zur Kontrollgruppe niedrigere Leistungen beim Mottier-Test, beim Kunstwörter-Nachsprechen, beim Trigramm-Test, bei der Wortspanne, beim Zahlen Nachsprechen vorwärts und beim Zahlen Nachsprechen rückwärts. Für das KunstwortNachsprechen, in welchem das Itemmaterial hinsichtlich zweier Dimensionen variiert wird, ist anzumerken, dass die Defizite bezüglich der Dimension Silbenanzahl gegenüber der Kontrollgruppe umso ausgeprägter sein sollten, je stärker die beeinträchtigende Materialvariation ausfällt. Bei der Variation des Faktors Verzerrtheitsgrad wird eine Ebenbürtigkeit der Leistung beider Gruppen oder ein vorübergehender Leistungsvorteil der lese-/rechtschreibschwachen gegenüber der Kontrollgruppe erwartet. Beim Mottier-Test wird die Dimension Silbenanzahl ebenfalls sukzeessive variiert, weshalb erwartet wird, dass auch hier die Defizite bezüglich der gegenüber der Kontrollgruppe umso ausgeprägter sind, je stärker die beeinträchtigende Materialvariation ausfällt.

Die Hypothese 1.b führt zu der wie folgt lautenden empirischen Vorhersage (eV 1.b):

Die Kinder der lese-/rechtschreibschwachen Gruppe zeigen im Vergleich zur Kontrollgruppe niedrigere Leistungen in den Tests der Würzburger Testbatterie, das heißt dem Laute Ersetzen, dem Restworte-Test, dem Laute Vertauschen und dem Laute Klassifizieren, sowie in den Tests des PET, das heißt dem Worte Ergänzen und dem Laute Verbinden.

Aus der Hypothese 1.1 leitet sich die empirische Vorhersage eV $1.1 \mathrm{ab}$ :

Die Kinder der lese-/rechtschreibschwachen Gruppe zeigen im Vergleich zur Kontrollgruppe niedrigere Leistungen beim Wortproduktionstest und beim Farben Benennen.

Die Kombination aller drei empirischen Vorhersagen entspricht der empirischen Vorhersage eV 1.0, die aus der $\mathrm{pH} 1.0$ abzuleiten ist. 


\subsubsection{AUDITIV-SENSORISCHE VERARBEITUNGSDEFIZITE}

In der psychologischen Hypothese 2.0 wird die Erwartung auditiv sensorischer Verarbeitungsschwächen bei lese-/rechtschreibschwachen Probanden im Vergleich zu Kontrollprobanden formuliert. Daraus lässt sich die empirische Vorhersage eV 2.0 ableiten:

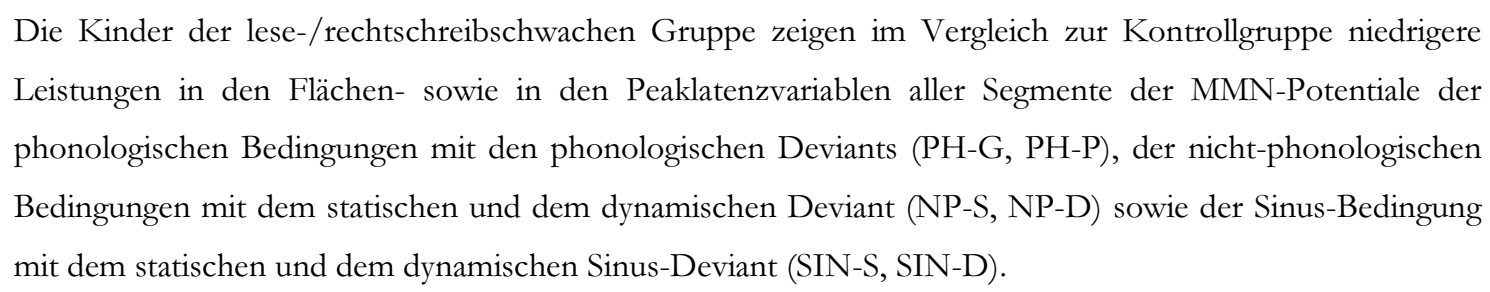

Die konkurrierenden Hypothesen 2.g, 2.s und 2.t untergliedern die Vorhersage eV 2.0. Sie betreffen das Auftreten von Verarbeitungsdefiziten im Sinne eines rasch temporalen ( $\mathrm{pH} 2 . \mathrm{t}$ ), eines generell auditiven ( $\mathrm{pH}$ 2.g) oder eines spezifisch sprachlichen Defizits ( $\mathrm{pH}$ 2.s).

Um die empirischen Vorhersagen $\mathrm{eV}$ 2.g, eV 2.s und eV 2.t jedoch mit der übergeordneten empirischen Vorhersage eV $2.0 \mathrm{zu}$ verbinden, muss die Konzeption der MMN-Bedingungen einbezogen werden. Die Reizkontraste waren so gestaltet worden, dass Muster von schlechteren Leistungen der lese-/rechtschreibschwachen Gruppe in bestimmten Bedingungen auf jeweils bestimmte Typen von Verarbeitungsdefiziten hinweisen. Die Zuordnung von MMNBedingungsmuster und Typus eines auditiv sensorischen Defizits ist in der folgenden Tabelle ersichtlich.

\begin{tabular}{|c|c|c|c|c|c|}
\hline \multicolumn{3}{|c|}{ auditiv sensorisches Verarbeitungsdefizit:: } & generell auditiv & spezifisch sprachlich & rasch temporal \\
\hline \multirow{4}{*}{$\begin{array}{l}\text { phono- } \\
\text { logischer } \\
\text { Standard }\end{array}$} & \multirow{2}{*}{$\begin{array}{c}\text { phonologische } \\
\text { Kontraste }\end{array}$} & PH-P & $\downarrow$ & $\downarrow$ & $\downarrow /=$ \\
\hline & & PH-G & $\downarrow$ & $\downarrow$ & $\downarrow /=$ \\
\hline & \multirow{2}{*}{$\begin{array}{l}\text { nicht-phonologische } \\
\text { Kontraste } \\
\text { (Frequenz) }\end{array}$} & statisch [NP-S] & $\downarrow$ & $=$ & $=$ \\
\hline & & dynamisch [NP-D] & $\downarrow$ & $=$ & $\downarrow$ \\
\hline \multirow{2}{*}{$\begin{array}{l}\text { Sinuston- } \\
\text { Standard }\end{array}$} & \multirow{2}{*}{$\begin{array}{l}\text { nicht-phonologische } \\
\text { Kontraste } \\
\text { (Frequenz) }\end{array}$} & statisch [SIN-S] & $\downarrow$ & $=$ & $=$ \\
\hline & & dynamisch [SIN-D] & $\downarrow$ & $=$ & $\downarrow$ \\
\hline $\begin{array}{c}\text { Tab. 10: } \\
\quad=/ \downarrow:\end{array}$ & \multicolumn{5}{|c|}{$\begin{array}{l}\text { Zuordnung von MMN-Bedingungsmustern zu Typen auditiv sensorischer Verarbeitungsdefizite. } \\
\text { Die Leistung in der entsprechenden MMN-Bedingung fällt bei lese-/rechtschreibschwachen Probanden genauso gut wie / } \\
\text { schlechter als bei Kontrollprobanden aus. }\end{array}$} \\
\hline
\end{tabular}


Die empirischen Vorhersagen sind damit wie folgt abzuleiten:

$\mathrm{eV} 2 . \mathrm{g}$

Im Falle des Vorliegens eines Defizits der generell auditiven Verarbeitung zeigen die Kinder der lese/rechtschreibschwachen Gruppe im Vergleich zur Kontrollgruppe Leistungsbeeinträchtigungen in Flächenoder Peaklatenzvariablen aller Typen von MMN-Bedingungen.

$\mathrm{eV} 2 . \mathrm{s}$

Im Falle des Vorliegens eines spezifisch sprachlichen Defizits der auditiven Verarbeitung zeigen die Kinder der lese-/rechtschreibschwachen Gruppe im Vergleich zur Kontrollgruppe Leistungsbeeinträchtigungen in Flächen- oder Peaklatenzvariablen der phonologischen MMN-Bedingungen (PH-P, PH-G).

$\mathrm{eV} 2 . \mathrm{t}$

Im Falle des Vorliegens eines Defizits der rasch temporalen Verarbeitung zeigen die Kinder der lese/rechtschreibschwachen Gruppe im Vergleich zur Kontrollgruppe Leistungsbeeinträchtigungen in Flächenoder Peaklatenzvariablen der MMN-Bedingungen, die kurzfristige Veränderungen beinhalten (NP-D, SIND). Leistungsbeeinträchtigungen in den phonologischen MMN-Bedingungen (PH-P, PH-G) sind möglich.

\subsubsection{ZUSAMMENHANG ZWISCHEN PHONOLOGISCHEN UND AUDITIV SENSORISCHEN VERARBEITUNGSDEFIZITEN}

Die psychologische Hypothese 3a beinhaltet die Erwartung eines Zusammenhangs zwischen phonologischen und auditiv sensorischen Verarbeitungsdefiziten bei der Lese-/Rechtschreibstörung. Daraus lässt sich die folgende empirische Vorhersage eV 3a ableiten:

Bei der Erklärung der Rechtschreibleistung weisen der Bereich der kognitionspsychologischen Verarbeitungsebene und der Bereich der neurophysiologischen Verarbeitungsebene gemeinsame Varianz auf. Konstrukte und neurophysiologische Ebene werden hierbei jeweils durch ein noch zu definierendes Aggregat aus optimal repräsentativen Variablen für das Konstrukt bzw. die Verarbeitungsebene konstituiert.

Im Kontext dieser empirischen Vorhersage steht ein Arbeitsauftrag zur ausführlichen Exploration dieses Zusammenhangs. Neben der Größenschätzung der Varianzerklärungsanteile an der Rechtschreibleistung für jede Verarbeitungsebene bzw. für jedes Konstrukt kann die Größenschätzung kombinierter Teilbereiche erfolgen, was nicht nur obige empirische Vorhersage bejaht oder verneint, sondern außerdem eine Vorstellung über die Struktur der Konstrukte / Verarbeitungsebenen innerhalb des Varianzraumes der Rechtschreibleistung vermittelt. In diesem Abschnitt gilt es weiterhin zu beantworten, ob die neurophysiologische 
Verarbeitungsebene Anteile der Rechtschreibleistung erklären kann, die von der Ebene der kognitionspsychologischen Verarbeitung nicht abgedeckt werden.

Wurden gemeinsame Varianzbereiche der Konstrukte bzw. Verarbeitungsebenen bei der Erklärung der Rechtschreibleistung nachgewiesen, soll mithilfe eines LISREL-Modells geprüft werden, ob die Konstrukte in einer zeitlichen und inhaltlich aufeinander aufbauenden Rangfolge zueinander stehen. Zur Rekapitulation der Rangfolge, wie sie die psychologische Hypothese $3 \mathrm{~b}$ formuliert, sei die entsprechende Abbildung hier noch einmal aufgeführt.

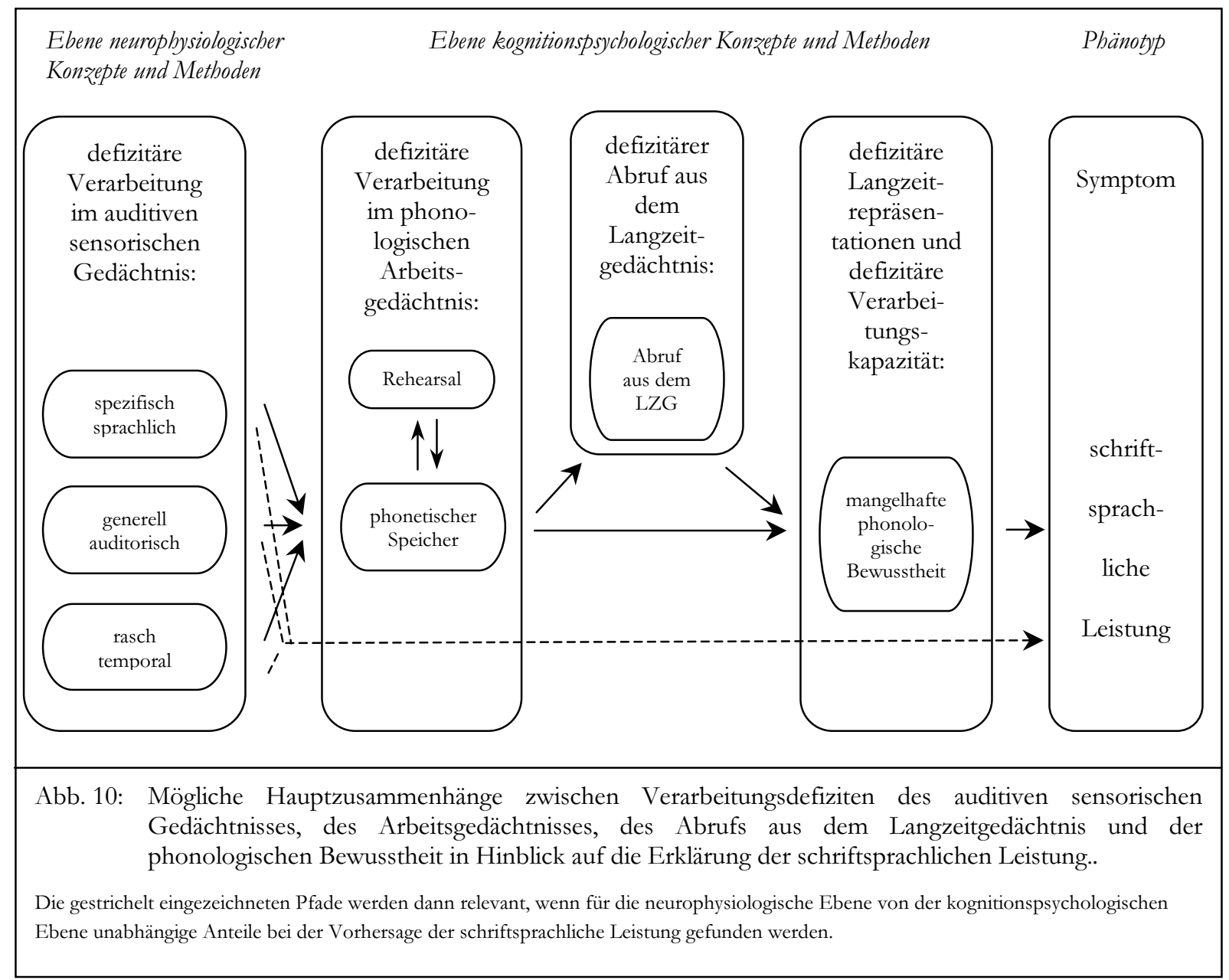

In einem derartigen Modell würde das EEG die basalste Ebene darstellen. Seine Einflüsse richteten sich -aufgrund der konzeptionellen Ähnlichkeit zwischen Merkmalen der MMN und dem phonetischen Speicher- in erster Linie auf das phonologische Arbeitsgedächtnis; direkte Einflüsse auf die Rechtschreibleistung sollen jedoch nicht ausgeschlossen werden, wenn die 
EEG-Variablen relativ zu den kognitionspsychologischen Konstrukten zusätzliche Rechtschreibvarianz erklären konnten. Gegebenenfalls werden die beiden Alternativmodelle berechnet. Der Einfluss des Arbeitsgedächtnis auf die Rechtschreibung wäre eher indirekter Natur und verliefe über den zuverlässig starken Prädiktor der Schriftsprache, der phonologischen Bewusstheit. Da die Zentrale Exekutive als Regulationseinheit des Arbeitsgedächtnis möglicherweise in engem und aktivem Zusammenhang zum Abruf aus dem Langzeitgedächtnis steht, wird eine Einflussnahme des Arbeitsgedächtnisses auf die Aktivierung des Langzeitgedächtnisses postuliert; abgerufene Langzeitrepräsentationen unterstützen wahrscheinlich die kognitiven Prozesse der phonologischen Bewusstheit, so dass für diese eine Einflussnahme vom Langzeitgedächtnis-Abruf erwartet wird.

Aus der psychologischen Hypothese $3 b$ ist damit die empirische Vorhersage $3 b$ wie folgt abzuleiten:

In einem LISREL-Modell lassen sich die untersuchten Konstrukte und Verarbeitungsebenen in den wie folgt abgebildeten Zusammenhang von Einflussnahmen stellen.

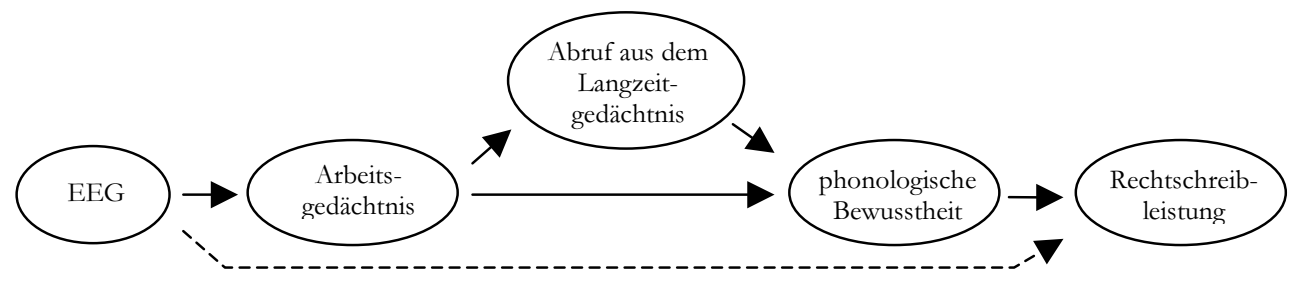

Ein weiterer positiver Pfad vom EEG auf die Rechtschreibleistung (gestrichelte Pfeillinie) ist dann erforderlich, wenn dem EEG-Bereich Varianzerklärungen zuzuschreiben sind, die durch die kognitionspsychologischen Variablen noch nicht abgedeckt wurden.

Welche Variablen die jeweiligen Ebenen bzw. Konstrukte konstituieren, wird an dieser Stelle nicht namentlich festgelegt: Es müssen in jedem Fall diejenigen sein, die im Rahmen der empirischen Vorhersage 3a die Agglomerate bildeten. 


\section{ERGEBNISSE}

Die Darstellung der Ergebnisse orientiert sich an der Ordnung der empirischen Vorhersagen und gliedert sich demzufolge in einen Abschnitt zu Lese-/Rechtschreibschwäche-spezifischen Verarbeitungsdefiziten der phonologischen Informationsverarbeitung, in einen Abschnitt zu Verarbeitungsdefiziten der auditiv sensorischen Informationsverarbeitung und in einen dritten Abschnitt, in welchem ein möglicher Zusammenhang zwischen den Verarbeitungsdefiziten der beiden Ebenen nachgewiesen und spezifiziert werden soll.

Die Berechnungen erfolgten, soweit nicht anders angegeben, mit dem Programm SPSS 10.

\subsection{GRUPPENUNTERSCHIEDE DER PHONOLOGISCHEN VERARBEITUNG}

Bei der folgenden Dokumentation der Ergebnisse zur phonologischen Verarbeitung ist jeweils ein Abschnitt einem Konstrukt der Triade phonologischer Verarbeitung gewidmet. Jeder dieser Abschnitte beinhaltet damit die Untersuchungen zu einer der empirischen Hypothesen (eV 1.a, $\mathrm{eV}$ 1.b, eV 1.1), welche der empirischen Hypothese 1.0 untergeordnet sind. Die Antwort auf die empirische Hypothese eV 1.0 ergibt sich damit aus dem Gesamtbild der drei Abschnitte.

\subsubsection{ERGEBNISSE DER TESTS ZUM ARBEITSGEDÄCHTNIS}

Die Ergebnisse zum Arbeitsgedächtnis werden in kleinen Abschnitten vorgestellt, in denen jeweils die Tests zusammengefasst sind, die dieselbe Arbeitsgedächtnis-Komponente untersuchen: Das Kunstwort-Nachsprechen und der Mottier-Test als Speicher-Variablen bilden einen Abschnitt, der Trigramm-Test, der die Rehearsal-Geschwindigkeit schätzt, einen weiteren, und die Wort- und Zahlenspannentests als Indikatoren der funktionalen Gesamtkapazität den letzten der drei.

Zur Ermittlung bedeutsamer Gruppenunterschiede wurden die Kennwerte in der Regel t-Tests unterzogen. Die optimale Auswertung des Kunstwort-Nachsprechens setzte sich von der der anderen Tests $a b$, da er zwei Dimensionen besitzt und eine varianzanalytische Herangehensweise dem am Besten gerecht wurde. Beim Mottier-Test sollte aufgrund seiner großen 
Ähnlichkeit zum Kunstworttest in einer der beiden Dimensionen, in der Auswertung analog verfahren werden, so dass die Ergebnisse der beiden Testtypen miteinander vergleichbar wurden.

Für Prüfung die aller Tests zum Arbeitsgedächtnis erschien das übliche Signifikanzniveau von $\alpha=.05$ angemessen.

\subsubsection{KUNSTWORT-NACHSPRECHEN UND MOTTIER-TEST}

\subsection{ERGEBNISSE DES KUNSTWORT-NACHSPRECHENS}

Eine Darstellung der deskriptiven Statistik des Kunstworte-Nachsprechens ist in Tabelle 11 ersichtlich. Das folgende Diagramm in Abbildung 11 visualisiert die Inhalte der Tabelle. Die Ergebnisse der Varianzanalyse sind in Tabelle $12 \mathrm{zu}$ sehen.

Da es bei der Bedingung der stark verzerrten Itemdarbietung in beiden Experimentalgruppen zu Bodeneffekten kam und Gruppenunterschiede folglich nicht zu beobachten wären (Ergebnisse nicht dargestellt), wurde diese Bedingung aus der Auswertung der Kunstwortaufgabe ausgeschlossen.

\begin{tabular}{|c|c|c|c|c|c|c|c|c|c|}
\hline & & \multicolumn{3}{|c|}{ KTR } & \multicolumn{3}{|c|}{ LRS } & \multicolumn{2}{|c|}{ KTR + LRS } \\
\hline & & $\begin{array}{l}\text { unver- } \\
\text { zerrte } \\
\text { KW }\end{array}$ & $\begin{array}{l}\text { verzerrte } \\
\text { KW }\end{array}$ & $\begin{array}{c}\text { Rand- } \\
\text { summe } \\
\text { KTR }\end{array}$ & $\begin{array}{l}\text { unver- } \\
\text { zerrte } \\
\text { KW }\end{array}$ & $\begin{array}{l}\text { verzerrte } \\
\text { KW }\end{array}$ & $\begin{array}{l}\text { Rand- } \\
\text { summe } \\
\text { LRS }\end{array}$ & $\begin{array}{c}\text { Rand- } \\
\text { summe } \\
\text { unverzerr- } \\
\text { te KW }\end{array}$ & $\begin{array}{c}\text { Rand- } \\
\text { summe } \\
\text { verzerrte } \\
\text { KW }\end{array}$ \\
\hline $\begin{array}{c}\text { zweisilbige } \\
\text { KW }\end{array}$ & $\begin{array}{l}\text { M IN Items] (max. 4) } \\
\mathrm{S}\end{array}$ & $\begin{array}{c}3.6 \\
.5 \\
\end{array}$ & $\begin{array}{c}2.9 \\
.7 \\
\end{array}$ & $\begin{array}{l}6.5 \\
1.0 \\
\end{array}$ & $\begin{array}{c}3.3 \\
.8 \\
\end{array}$ & $\begin{array}{c}3.4 \\
.6 \\
\end{array}$ & $\begin{array}{l}6.7 \\
1.2 \\
\end{array}$ & 6.9 & 6.3 \\
\hline $\begin{array}{c}\text { dreisilbige } \\
\text { KW }\end{array}$ & $\begin{array}{l}\text { M [N Items] (max. 4) } \\
\mathrm{S}\end{array}$ & $\begin{array}{c}3.7 \\
.5\end{array}$ & $\begin{array}{c}2.7 \\
.7\end{array}$ & $\begin{array}{l}6.4 \\
1.1\end{array}$ & $\begin{array}{l}3.1 \\
1.1\end{array}$ & $\begin{array}{l}2.1 \\
1.1\end{array}$ & $\begin{array}{l}5.2 \\
1.6\end{array}$ & 6.8 & 4.8 \\
\hline $\begin{array}{c}\text { viersilbige } \\
\text { KW }\end{array}$ & $\begin{array}{l}\mathrm{M}[\mathrm{N} \text { Items] (max. 4) } \\
\mathrm{S}\end{array}$ & $\begin{array}{c}3.5 \\
.6\end{array}$ & $\begin{array}{l}2.3 \\
1.1\end{array}$ & $\begin{array}{l}5.8 \\
1.3\end{array}$ & $\begin{array}{c}2.9 \\
1.0\end{array}$ & $\begin{array}{c}1.2 \\
.9\end{array}$ & $\begin{array}{l}4.0 \\
1.5\end{array}$ & 6.4 & 3.5 \\
\hline $\begin{array}{l}\text { Rand- } \\
\text { summe }\end{array}$ & $\begin{array}{l}\text { M IN Itemsl (max. 12) } \\
\mathrm{S}\end{array}$ & $\begin{array}{c}10.8 \\
1.1\end{array}$ & $\begin{array}{l}7.9 \\
2.1\end{array}$ & & $\begin{array}{l}9.3 \\
2.1\end{array}$ & $\begin{array}{l}6.7 \\
1.6\end{array}$ & & 20.1 & 14.6 \\
\hline $\begin{array}{l}\text { Rand- } \\
\text { summe }\end{array}$ & $\begin{array}{l}\text { M IN Itemsl (max. 24) } \\
\text { S }\end{array}$ & \multicolumn{2}{|c|}{18.7} & & \multicolumn{2}{|c|}{$\begin{array}{c}15.9 \\
3.4\end{array}$} & & \multicolumn{2}{|c|}{34.7} \\
\hline
\end{tabular}

Tab. 11: Deskriptive Statistik des Kunstwort-Nachsprechens

KTR: Kontrollgruppe; LRS: lese-/ rechtschreibschwache Gruppe; KW: Kunstworte; M: Mittelwert; S: Standardabweichung; N: Anzahl 


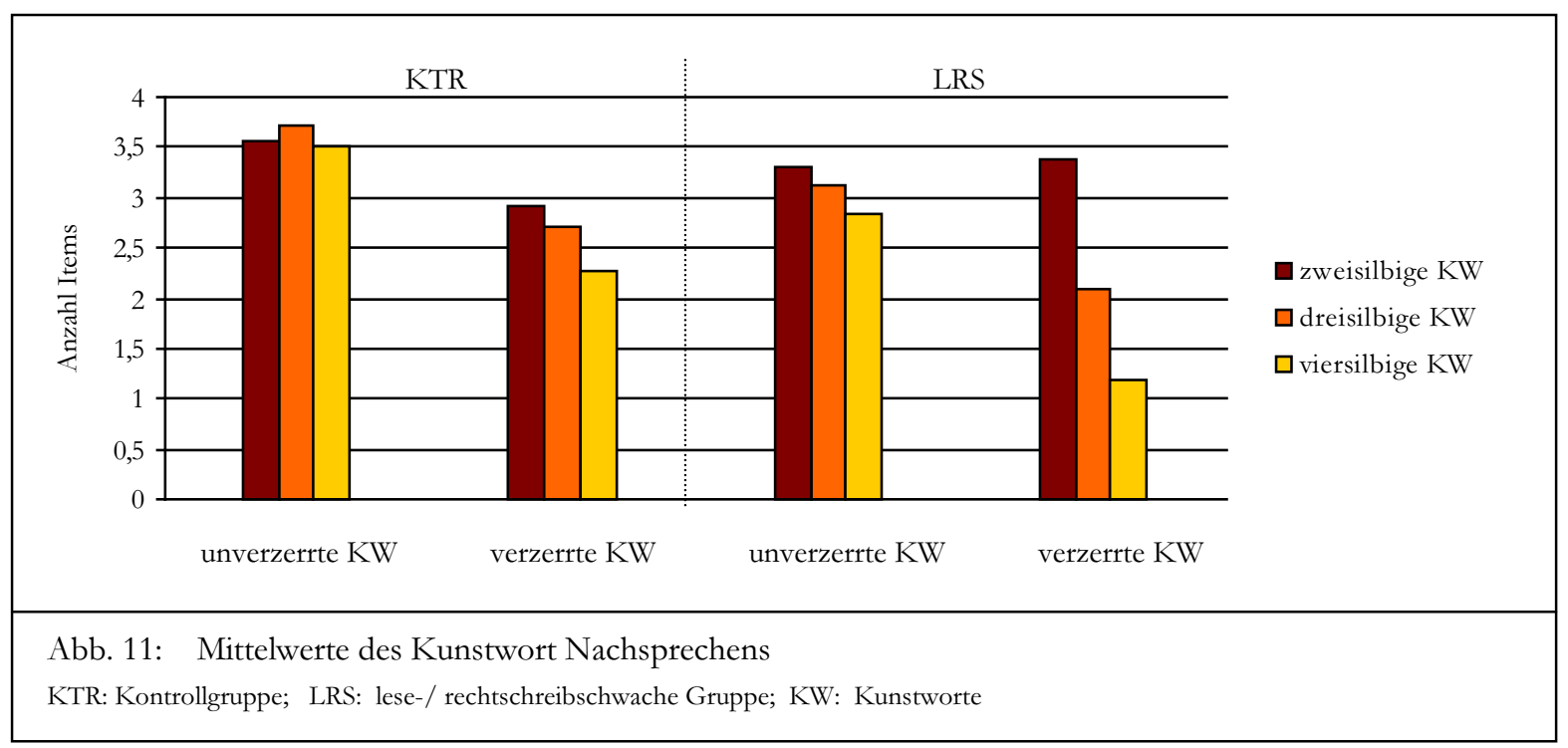

\begin{tabular}{|c|c|c|c|c|c|}
\hline & df & $\mathbf{F}$ & sign. & $\eta^{2}$ & gemäß eV 1.a? \\
\hline HE GRUPPE & 1 & 10.6 & .002 & .177 & $\sqrt{ }$ \\
\hline für Silbenanzahl = 2 für unverzerrte Items & 1 & 1.8 & .182 & .036 & \\
\hline für Silbenanzahl = 2 für verzerrte Items & 1 & 6.1 & .017 & .111 & \\
\hline für Silbenanzahl = 3 für unverzerrte Items & 1 & 6.4 & .015 & .115 & \\
\hline für Silbenanzahl = 3 für verzerrte Items & 1 & 6.3 & .015 & .114 & \\
\hline für Silbenanzahl = 4 für unverzerrte Items & 1 & 8.4 & .006 & .147 & \\
\hline für Silbenanzahl = 4 für verzerrte Items & 1 & 15.7 & .000 & .243 & \\
\hline HE SILBENANZAHL & 2 & 35.3 & .000 & .418 & \\
\hline für Verzerrung $=0$ bei KTR & 2 & 1.1 & .338 & .044 & \\
\hline für Verzerrung $=0$ bei LRS & 2 & 2.1 & .133 & .078 & \\
\hline für Verzerrung $=1$ bei KTR & 2 & 7.4 & .002 & .236 & \\
\hline für Verzerrung $=1$ bei LRS & 2 & 44.7 & .000 & .641 & \\
\hline HE VERZERRUNG & 1 & 121.8 & .000 & .713 & \\
\hline für Silbenanzahl $=2$ bei KTR & 1 & 20.9 & .000 & .465 & \\
\hline für Silbenanzahl $=3$ bei KTR & 1 & 50.0 & .000 & .676 & \\
\hline für Silbenanzahl $=4$ bei KTR & 1 & 32.3 & .000 & .574 & \\
\hline für Silbenanzahl $=2$ bei LRS & 1 & .3 & .603 & .011 & \\
\hline für Silbenanzahl $=3$ bei LRS & 1 & 14.3 & .001 & .364 & \\
\hline für Silbenanzahl $=4$ bei LRS & 1 & 52.5 & .000 & .677 & \\
\hline IA GRUPPE * SILBENANZAHL & 2 & 13.3 & .000 & .213 & $\sqrt{ }$ \\
\hline für Verzerrung $=0$ & 2 & 1.4 & .249 & .028 & \\
\hline für Verzerrung $=1$ bei Silbenanzahl 2 und 3 & 1 & 13.4 & .001 & .215 & \\
\hline für Verzerrung $=1$ bei Silbenanzahl 3 und 4 & 1 & 1.8 & .185 & .036 & \\
\hline \multicolumn{6}{|l|}{ IA GRUPPE * VERZERRUNG } \\
\hline für Silbenanzahl = 2 & 1 & 12.5 & .001 & .204 & $\sqrt{ }$ \\
\hline für Silbenanzahl = 3 & 1 & .015 & .903 & .000 & \\
\hline für Silbenanzahl = 4 & 1 & 1.7 & .197 & .034 & \\
\hline IA GRUPPE * SILBE * VERZERRUNG & 2 & 5.5 & .007 & .188 & \\
\hline
\end{tabular}

Tab. 12: Varianzanalyse des Kunstwort-Nachsprechens.

Die Auflistung der Teileffekte dient der Lokalisierung der Haupteffekte. Durch roten Druck hervorgehoben sind die p-Werte erwartungsgemäß gerichteter und auf dem 5\%-Niveau signifikanter (Teil-) Effekte.

HE: Haupteffekt; IA: Interaktionseffekt; KTR: Kontrollgruppe; LRS: Lese-/rechtschreibschwache Gruppe; Verzerrung = 0 / $1:$ Die dargebotenen Items waren unverzerrt / verzerrt; $\sqrt{ }$ : Effekt im Sinne der empirischen Vorhersage. 


\section{Haupteffekt Gruppe}

Im Mittel schnitten die Kontrollprobanden bei der Wiedergabe der Kunstwörter besser ab als die lese-/rechtschreibschwachen Kinder. Der Gruppeneffekt beruhte insbesondere auf der Minderleistung der klinischen Gruppe bei der Reproduktion drei- und viersilbiger Items.

\section{Haupteffekt Silbenanzahl und Interaktionseffekt Gruppe * Silbenanzahl}

Die Steigerung der Silbenanzahl unverzerrter Items meisterten die Probanden der Kontrollgruppe ohne Leistungseinbußen. Da die Leistung aller Silbenanzahlen jeweils nahe dem Maximum lag, ist es wahrscheinlich, dass es sich hier um einen Deckeneffekt handelt. Die relativ geringe Standardabweichung von .5 bis .6 in diesen Bedingungen im Vergleich zu den Standardabweichungen der anderen Bedingungen von .6 bis 1.1 mag ebenfalls ein Indiz auf einen Deckeneffekt sein. Dennoch spricht die Tatsache, dass die LRS-Gruppe unter gleichen Bedingungen bereits eine fallende Leistungstendenz zeigte (wenn diese auch noch nicht signifikant war), dafür, die einfachsten Bedingungen dieses Tests nicht zu erschweren.

Ein Silbenhaupteffekt zeugte allerdings vom insgesamt dominierenden leistungsmindernden Einfluss der Silbensteigerung bei den verzerrten Items. In beiden Gruppen sank die Leistung signifikant; bei der lese-/rechtschreibschwachen Gruppe war dieser Effekt besonders drastisch, da die Reproduktion zweisilbiger Items etwa auf dem Niveau der maximalen Leistung gesunder Probanden bei zweisilbigen Kunstworten, die Reproduktion viersilbiger Items jedoch unter der Minimalleistung der Kontrollgruppe lag.

Der immense Leistungsunterschied der Lese-/rechtschreibschwachen bei der Reproduktion dreisilbiger versus zweisilbiger verzerrter Items von 1.3 Items verglichen mit der minimalen Reproduktionsminderung der Kontrollprobanden von 0.2 Items war die Ursache für den signifikanten Interaktionseffekt der Silbenerhöhung mit dem Gruppenfaktor. Dieser Interaktionseffekt wurde auch bezogen auf den Gesamttest signifikant.

(Da bei unverzerrten Items in keiner Gruppe ein Silbeneffekt auftrat, ist hier folgerichtig kein Interaktionseffekt vorhanden.)

\section{Haupteffekt Verzerrung und Interaktionseffekt Gruppe * Verzerrung}

Die Verzerrung der Item-Darbietung übte einen beträchtlichen Einfluss auf die Reproduktionsleistung aus, so dass es zu einem erwartungsgemäßen und signifikanten Haupteffekt kam. Dieser fand sich sowohl bei den Kontroll- als auch bei den lese-/rechtschreibschwachen Probanden in beinahe jeder Itemlängenbedingung wieder. 
Die Ausnahme bildeten die zweisilbigen Kunstworte: Lese-/Rechtschreibschwache reproduzierten verzerrte zweisilbige Items ebenso gut wie unverzerrte zweisilbige Items. Die zweisilbig verzerrten Worte waren von Lese-/rechtschreibschwachen zudem signifikant besser reproduziert worden als von gesunden Probanden $(F=6.1)$. Dieser paradoxe Effekt ist verantwortlich für einen signifikanten Interaktionseffekt der Verzerrtheit mit dem Gruppenfaktor bei der Silbenanzahl 2. Bei den längersilbigen Items trat kein weiterer Interaktionseffekt auf.

Auf der Signifikanz der Interaktionseffekte des Gruppenfaktors mit der Silbenanzahl sowie dem Verzerrungsgrad bei zweisilbigen Items beruht die Signifikanz des Tripelinteraktionseffektes.

\subsection{ZUSAMMENFASSUNG UND BEURTEILUNG HINSICHTLICH DER EMPIRI- SCHEN VORHERSAGE 1.A}

Die Gesamtreproduktionsleistung beim Kunstwort-Nachsprechen fiel für lese-/rechtschreibschwache Probanden schlechter als für gesunde Probanden aus (Haupteffekt Gruppe). Hierbei war die Beeinträchtigung der klinischen Gruppe umso größer, je stärker die Materialvariation hinsichtlich der Dimension Silbenanzahl wurde (Interaktionseffekt Gruppe*Silbenanzahl). Die Variation des Verzerrungsgrads der Itemdarbietung führte zu einem zweiten Interaktionseffekt mit dem Gruppenfaktor; dessen paradoxe Natur hinsichtlich eines Leistungsvorteils der lese/rechtschreibschwachen Gruppe war erwartet worden.

Der Haupteffekt Gruppe sowie die Interaktionseffekte von Gruppe und Silbenanzahl bzw. Gruppe und Verzerrungsgrad sind demnach Resultate, die im Sinne der empirischen Vorhersage 1.a ausfielen.

Der Gruppenhaupteffekt sowie der Interaktionseffekt 'Gruppe*Silbenanzahl' lassen auf ein vermindertes Speichervolumen des phonetischen Speichers schließen. Eine mögliche weitergehende Interpretation des Interaktionseffekts könnte eine Annahme einer verminderten Verarbeitungspräzision innerhalb des phonetischen Speichers sein: Geht man davon aus, dass der sprachliche Input bei Lese-/rechtschreibschwachen stets mit einer gewissen Unschärfe versehen ist und die Kinder so im Laufe ihres Lebens Übung im Schließen auf die richtige Form entwickeln könnten (oder auch sehr geschickt im Vertuschen ihrer Unsicherheiten bei der Wiedergabe werden könnten), so müssten die Wiedergabeleistungen lese-/rechtschreibschwacher Kinder bei verzerrten Worten besser sein als die gesunder Kinder. Diese Überlegenheit würde aber in dem Augenblick in eine Unterlegenheit wechseln, in welchem die Silbenlänge die Speicherkapazität lese-/rechtschreibschwacher Kinder überschreitet. Dieses Bild zeichnen die Befunde zum Kunstwort-Nachsprechen. 


\subsection{ERGEBNISSE DES MOTTIER-TESTS}

Die Ergebnisse des Mottier-Tests sind in folgenden Abbildungen dargestellt: Tabelle 13 enthält die Mittelwerte, Standardabweichungen und Randsummen; das Diagramm unter der Tabelle veranschaulicht die Mittelwerte. Die Ergebnisse der Varianzanalyse sind in Tabelle 14 aufgelistet.

\begin{tabular}{|c|c|c|c|c|c|}
\hline & & KTR & LRS & $\begin{array}{l}\text { Rand- } \\
\text { summe }\end{array}$ & \multirow{7}{*}{$\begin{array}{l}\text { Tab. 13: Mittelwerte, } \\
\text { Standardabweichungen } \\
\text { und Randsummen des } \\
\text { Mottier-Tests. } \\
\text { KTR: Kontrollgruppe; } \\
\text { LRS: lese-/ rechtschreibschwa- } \\
\text { che Gruppe }\end{array}$} \\
\hline $\begin{array}{c}\text { zweisilbige } \\
\text { Items }\end{array}$ & $\begin{array}{l}\text { M IN Itemsl (max. 6) } \\
\mathrm{S}\end{array}$ & $\begin{array}{l}\mathbf{6 . 0} \\
0.2 \\
\end{array}$ & $\begin{array}{l}\mathbf{5 . 7} \\
0.5 \\
\end{array}$ & 11.7 & \\
\hline $\begin{array}{l}\text { dreisilbige } \\
\text { Items }\end{array}$ & $\begin{array}{l}\text { M [N Items] (max. 6) } \\
\mathrm{S}\end{array}$ & $\begin{array}{l}\mathbf{5 . 2} \\
0.8 \\
\end{array}$ & $\begin{array}{l}5.1 \\
0.9 \\
\end{array}$ & 10.3 & \\
\hline $\begin{array}{l}\text { viersilbige } \\
\text { Items }\end{array}$ & $\begin{array}{l}\text { M [N Items] (max. 6) } \\
\mathrm{S}\end{array}$ & $\begin{array}{l}\mathbf{5 . 4} \\
1.0 \\
\end{array}$ & $\begin{array}{l}4.4 \\
1.3 \\
\end{array}$ & 9.8 & \\
\hline $\begin{array}{l}\text { fünfsilbige } \\
\text { Items }\end{array}$ & $\begin{array}{l}\text { M [N Items] (max. 6) } \\
\mathrm{S}\end{array}$ & $\begin{array}{l}4.5 \\
1.4 \\
\end{array}$ & $\begin{array}{r}2.3 \\
1.8 \\
\end{array}$ & 6.8 & \\
\hline $\begin{array}{l}\text { sechssilbige } \\
\text { Items }\end{array}$ & $\begin{array}{l}\mathrm{M} \text { [N Items] (max. 6) } \\
\mathrm{S}\end{array}$ & $\begin{array}{l}3.3 \\
1.5\end{array}$ & $\begin{array}{l}1.7 \\
1.6\end{array}$ & 5.0 & \\
\hline Gesamt & $\begin{array}{l}\text { M IN Itemsl (max. 36) } \\
\text { S }\end{array}$ & $\begin{array}{c}24.4 \\
1.5\end{array}$ & $\begin{array}{c}19.2 \\
4.2\end{array}$ & 43.6 & \\
\hline
\end{tabular}

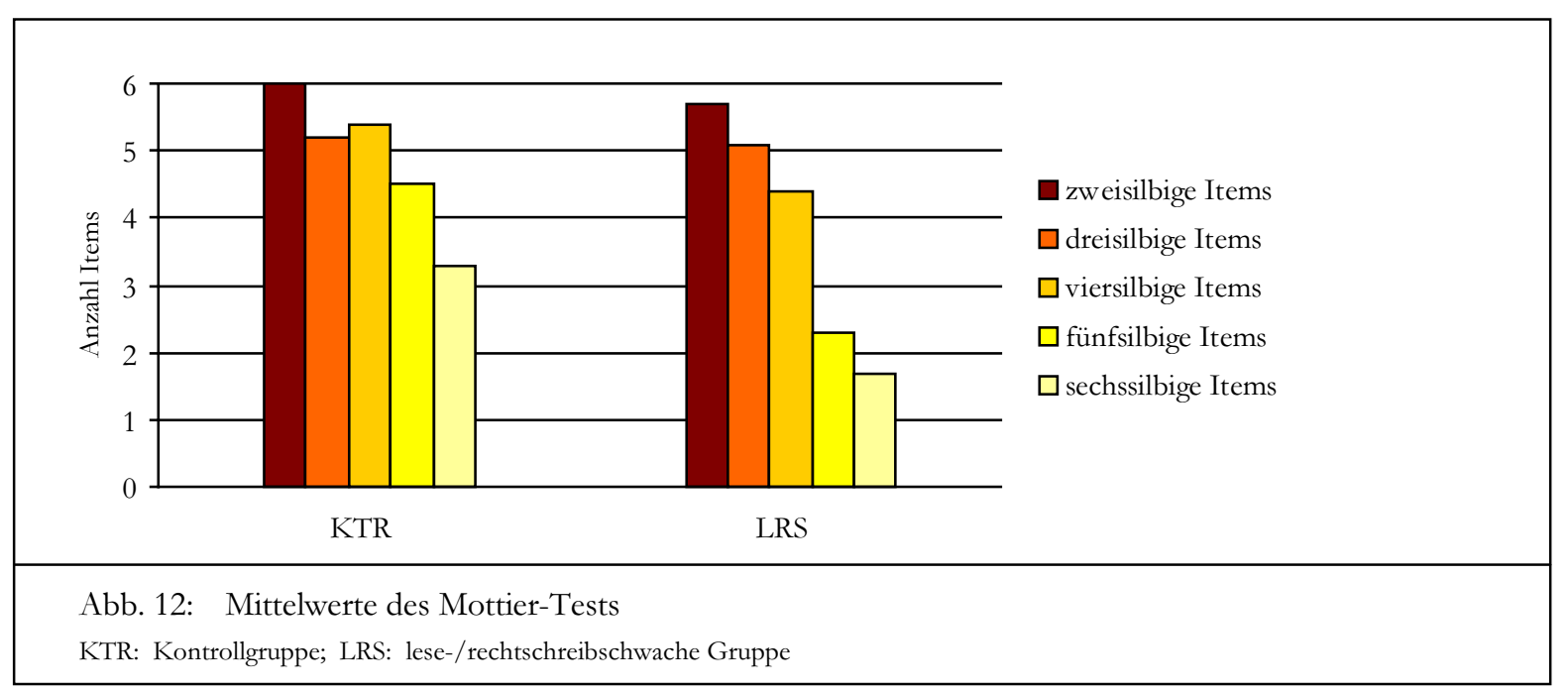




\begin{tabular}{|c|c|c|c|c|c|}
\hline & df & $\mathbf{F}$ & sign. & $\eta^{2}$ & gemäß eV 1.a? \\
\hline HE GRUPPE & 1 & 21.7 & .000 & .970 & $\sqrt{ }$ \\
\hline für Silbenanzahl = 2 & 1 & 4.1 & .049 & .077 & \\
\hline für Silbenanzahl = 3 & 1 & .4 & .514 & .009 & \\
\hline für Silbenanzahl = 4 & 1 & 8.7 & .005 & .151 & \\
\hline für Silbenanzahl = 5 & 1 & 24.7 & .000 & .336 & \\
\hline für Silbenanzahl = 6 & 1 & 13.4 & .001 & .214 & \\
\hline HE SILBENANZAHL & 4 & $\overline{94.4}$ & .000 & .659 & \\
\hline für Silbenanzahl = 2 und 3 bei KTR & 1 & 16.3 & .000 & .405 & \\
\hline für Silbenanzahl = 3 und 4 bei KTR & 1 & .4 & .527 & .017 & \\
\hline für Silbenanzahl = 4 und 5 bei KTR & 1 & 16.0 & .001 & .399 & \\
\hline für Silbenanzahl $=5$ und 6 bei KTR & 1 & 33.2 & .000 & .581 & \\
\hline für Silbenanzahl = 2 und 3 bei LRS & 1 & 15.5 & .001 & .383 & \\
\hline für Silbenanzahl = 3 und 4 bei LRS & 1 & 5.6 & .026 & .182 & \\
\hline für Silbenanzahl = 4 und 5 bei LRS & 1 & 47.6 & .000 & .656 & \\
\hline für Silbenanzahl $=5$ und 6 bei LRS & 1 & 2.5 & .124 & .092 & \\
\hline IA GRUPPE * SILBENANZAHL & 4 & $\overline{9.6}$ & .000 & .164 & $\sqrt{ }$ \\
\hline für Silbenanzahlen 2 und 3 & 1 & .1 & .787 & .002 & \\
\hline für Silbenanzahlen 3 und 4 & 1 & 4.7 & .034 & .088 & \\
\hline für Silbenanzahlen 4 und 5 & 1 & 9.9 & .003 & .169 & \\
\hline für Silbenanzahlen 5 und 6 & 1 & 2.7 & .105 & .053 & \\
\hline \multicolumn{6}{|c|}{$\begin{array}{l}\text { Tab. 14: Varianzanalyse des Mottier-Tests. } \\
\text { Die aufgeführten Teileffekte dienen der Lokalisierung der Haupteffekte. Durch roten Druck hervorgehoben sind die p-Werte } \\
\text { erwartungsgemäß gerichteter und auf dem 5\%-Niveau signifikanter (Teil-) Effekte. } \\
\text { HE: Haupteffekt; IA: Interaktionseffekt; KTR: Kontrollgruppe; LRS: Lese-/rechtschreibschwache Gruppe; V: Effekt im Sinne der } \\
\text { empirischen Vorhersage. }\end{array}$} \\
\hline
\end{tabular}

\section{Haupteffekt Gruppe}

Im Durchschnitt fiel die Reproduktionsleistung der lese-/rechtschreibschwachen Gruppe beim Mottier-Test schlechter als die der Kontrollgruppe aus. Dieser Leistungsnachteil wurde bei längersilbigen Items, das heißt ab einer Itemlänge von vier Silben, konsistent statistisch bedeutsam, wobei die Differenz bei fünfsilbigen Items besonders drastisch ausfiel (2.2 Items zu 1 bzw. 1.6 Items bei vier- und sechssilbigen Items).

\section{Haupteffekt Silbenanzahl}

In beiden Gruppen bewirkte die Steigerung der Silbenzahl bei beinahe jedem Steigerungsschritt und damit auch global ein signifikantes Absinken der Reproduktionsleistung. In der lese/rechtschreibschwachen Gruppe sank die Wiedergabeleistung fünfsilbiger gegenüber viersilbiger Pseudoworte um etwa den dreifachen Betrag (2.1 Items), den Längensteigerung um eine Silbe ansonsten bewirkt hatte (.63 Items). Bei den Kontrollprobanden trat kein derartig 
abrupter Leistungsabfall auf. Das Gegenteil war der Fall: viersilbige Items wurden von gesunden Kinder ebenso gut wie dreisilbige Items bewältigt.

\section{Interaktionseffekt Gruppe*Silbenanzahl}

Diese Leistungkonstanz gesunder Probanden zwischen drei- und viersilbigen Items im Vergleich zu einem signifikanten Absinken der Leistung lese-/rechtschreibschwacher Kinder war verantwortlich für das Signifikant-Werden des Interaktionsteileffektes der Silbenzahl mit dem Gruppenfaktor bei den Silbenanzahlen 3 und 4. Den zweiten der beiden signifikanten Interaktionsteileffekte bedingte die große Stufe zwischen der Reproduktion vier- und fünfsilbiger Items bei der LRS-Gruppe, die einem moderaten Leistungsverlust in der Kontrollgruppe (.9 Items) gegenüberstand.

\subsection{ZUSAMMENFASSUNG UND BEURTEILUNG HINSICHTLICH DER EMPIRI- SCHEN VORHERSAGE 1.A}

Im Mottier-Test wurde ebenso wie im Kunstworte-Test deutlich, dass die Reproduktionsfähigkeit lese-/rechtschreibschwacher Kinder gegenüber der von Kontrollkindern beeinträchtigt war (Haupteffekt Gruppe) und einer Steigerung der Silbenzahl weniger gut standhält als die von Kontrollkindern (Interaktionseffekt Gruppe*Silbenanzahl). Auch dieser Test bestätigte hiermit die Vermutung eines schwächeren Arbeitsgedächtnis-Speichers lese-/rechtschreibschwacher Personen.

Diese Ergebnisse entsprachen den Erwartungen aus der empirischen Vorhersage 1.a.

Neben Unterschieden im Detail resultierten beim Kunstwort-Nachsprechen und beim MottierTest demzufolge dieselben Haupt- und Interaktionseffekte: Gemäß beider Tests reproduzieren lese-/rechtschreibschwache Kinder Pseudoworte schlechter als gesunde Kinder, beide Tests riefen den Wortlängeneffekt hervor, und bei beiden Tests waren die Auswirkungen der Steigerung der Silbenanzahl bei Lese-/rechtschreibschwachen drastischer als bei der Kontrollgruppe, was die vermutete schwächere Speicherfähigkeit im Arbeitsgedächtnis lese/rechtschreibschwacher Probanden anzeigte. 


\subsubsection{TRIGRAMME}

Folgende Tabelle gibt das Ergebnis und die statistische Auswertung des Trigramm-Tests wieder. Es wurde deutlich, dass die lese-/rechtschreibschwachen Probanden signifikant mehr Zeit benötigten, um die Worttripel aufzusagen. Dies entsprach der Erwartung aus der empirischen Vorhersage 1.a und ließ einen Rückschluss auf ein langsameres Rehearsal in der phonologischen Schleife lese-/rechtschreibschwacher Personen zu.

\begin{tabular}{|l|c|c|c|c|c|c|c|}
\hline & KTR & LRS & df & $\mathbf{t}$ & $\mathbf{p}$ & $\eta^{\mathbf{2}}$ & gemäß eV 1.a? \\
\hline $\mathrm{M}[\mathrm{sek}]$ & $\mathbf{9 . 6}$ & $\mathbf{1 2 . 6}$ & 4.8 & 23.0 & .000 & .320 & $\sqrt{ }$ \\
$\mathrm{S}$ & 1.7 & 2.6 & & & & & \\
\hline
\end{tabular}

Tab. 15: Deskriptive und t-Test-statistische Auswertung des Trigramm-Tests.

KTR: Kontrollgruppe; LRS: lese-/rechtschreibschwache Gruppe; ل: Effekt im Sinne der empirischen Vorhersage; der p-Wert ist durch einen roten Druck hervorgehoben, da er sich unter der Signifikanzgrenze von von $\alpha \leq .05$ befindet.

\subsubsection{GEDÄCHTNISSPANNEN-TESTS}

Tabelle 16 listet die Ergebnisse sowie die statistische Auswertung des Wort- und Zahlenspannen-Tests auf.

\begin{tabular}{|c|c|c|c|c|c|c|c|c|}
\hline & & KTR & LRS & df & $\mathbf{t}$ & $\mathrm{p}$ & $\eta^{2}$ & gemäß eV 1.a? \\
\hline Wortspanne & $\begin{array}{l}\text { M [Spanne] } \\
\mathrm{S}\end{array}$ & $\begin{array}{c}4.4 \\
.5\end{array}$ & $\begin{array}{c}3.9 \\
.5\end{array}$ & 1 & 3.1 & .003 & .166 & $\sqrt{ }$ \\
\hline $\begin{array}{c}\text { Zahlenspanne } \\
\text { vorwärts }\end{array}$ & $\begin{array}{l}\text { M [Spanne] } \\
\mathrm{S}\end{array}$ & $\begin{array}{c}\mathbf{5 . 0} \\
.8\end{array}$ & $\begin{array}{c}4.3 \\
.6\end{array}$ & 1 & 3.4 & .001 & .197 & $\sqrt{ }$ \\
\hline $\begin{array}{c}\text { Zahlenspanne } \\
\text { rückwärts }\end{array}$ & $\begin{array}{l}\mathrm{M} \text { [Spanne] } \\
\mathrm{S}\end{array}$ & $\begin{array}{c}3.7 \\
.9\end{array}$ & $\begin{array}{c}3.1 \\
.6\end{array}$ & 1 & 2.8 & .008 & .136 & $\sqrt{ }$ \\
\hline
\end{tabular}

Tab. 16: Deskriptive und t-Test-statistische Auswertung der Spannentests.

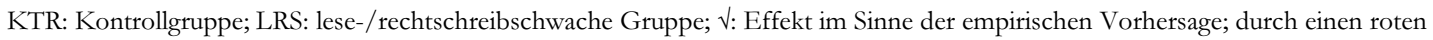
Druck hervorgehoben sind $\mathrm{p}$-Werte, die sich innerhalb der Signifikanzgrenze von $\alpha \leq .05$ befinden.

Je nach Spannentyp wurden unterschiedlich hohe Werte erzielt, was offenbar differierende Aufgabenschwierigkeitsgrade indiziert. Innerhalb einer Aufgabe ergab sich jedoch stets dasselbe Bild: Die Spanne lese-/rechtschreibschwacher Probanden blieb signifikant hinter der gesunder Versuchspersonen zurück. Dies ist nicht nur konsistent mit der empirischen Vorhersage eV 1.a bzw. der aus der Theorie resultierenden Erwartung einer eingeschränkten funktionalen 
Gesamkapazität der phonologischen Schleife -für was die Spannenwerte stehen-, sondern auch mit den bis eben vorgestellten Tests, da diese als Indikatoren der Teilmodule der phonologischen Schleife sich jeweils als ebenfalls eingeschränkt erwiesen haben.

\subsubsection{BEURTEILUNG DER EMPIRISCHEN VORHERSAGE 1.A}

Alle zur empirischen Vorhersage 1.a gehörigen Tests erbrachten Resultate im Sinne der Vorhersage. EV 1.a. hat sich demzufolge bestätigt.

\subsubsection{ERGEBNISSE DER TESTS ZUR PHONOLOGISCHEN BEWUSSTHEIT}

Die sechs Tests zur phonologischen Bewusstheit lassen sich in vier Tests zur phonologischen Bewusstheit im engeren Sinne und zwei Tests zur phonologischen Bewusstheit im weiteren Sinne einteilen. Die Ergebnisse aller Tests werden gemeinsam dargestellt. Diese und die statistische Auswertung der Tests zeigt Tabelle 17, wo auch die Zuordnung der Tests zu einer der beiden Kategorien der phonologischen Bewusstheit ersichtlich ist. Die statistische Prüfung erfolgte anhand von t-Tests; als Signifikanzniveau wurde $\alpha=.05$ angelegt.

\begin{tabular}{|c|c|c|c|c|c|c|c|c|}
\hline & & KTR & LRS & df & $\mathbf{t}$ & $\mathrm{p}$ & $\eta^{2}$ & gemäß eV 1.b? \\
\hline $\begin{array}{l}\text { Laute Ersetzene } \\
\text { (Wzbg.) }\end{array}$ & $\begin{array}{l}\mathrm{M}[\mathrm{N} \text { Items }](\max .15) \\
\mathrm{S}\end{array}$ & $\begin{array}{c}\mathbf{1 3 . 6} \\
1.4\end{array}$ & $\begin{array}{l}8.7 \\
3.5\end{array}$ & 1 & 6.5 & .000 & .460 & $\sqrt{ }$ \\
\hline $\begin{array}{l}\text { Restworte }^{\mathbf{e}} \\
\text { (Wzbg.) }\end{array}$ & $\begin{array}{l}\mathrm{M}[\mathrm{N} \text { Items }](\max .15) \\
\mathrm{S}\end{array}$ & $\begin{array}{c}12.7 \\
1.9\end{array}$ & $\begin{array}{l}8.7 \\
3.2\end{array}$ & 1 & 5.5 & .000 & .380 & $\sqrt{ }$ \\
\hline 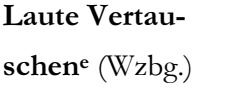 & $\begin{array}{l}\mathrm{M}[\mathrm{N} \text { Items }](\max .15) \\
\mathrm{S}\end{array}$ & $\begin{array}{c}13.9 \\
1.0\end{array}$ & $\begin{array}{l}9.7 \\
3.5\end{array}$ & 1 & 5.7 & .000 & .402 & $\sqrt{ }$ \\
\hline $\begin{array}{l}\text { Laute Verbin- } \\
\text { den }^{\text {e }} \text { (PET) }\end{array}$ & $\begin{array}{l}\mathrm{M}[\mathrm{N} \text { Items }] \text { (max. 33) } \\
\mathrm{S}\end{array}$ & $\begin{array}{c}23.8 \\
3.0\end{array}$ & $\begin{array}{c}19.6 \\
4.4\end{array}$ & 1 & 4.0 & .000 & .244 & $\sqrt{ }$ \\
\hline $\begin{array}{l}\text { Laute Klassifi- } \\
\text { zierenw (Wzbg.) }^{\text {w }}\end{array}$ & $\begin{array}{l}\mathrm{M}[\mathrm{N} \text { Items] }(\max .12) \\
\mathrm{S}\end{array}$ & $\begin{array}{l}8.9 \\
1.8\end{array}$ & $\begin{array}{l}5.8 \\
2.4\end{array}$ & 1 & 5.3 & .000 & .364 & $\sqrt{ }$ \\
\hline $\begin{array}{l}\text { Worte Ergän- } \\
\text { zenw }^{\text {wET})}\end{array}$ & $\begin{array}{l}\mathrm{M}[\mathrm{N} \text { Items }](\max .36) \\
\mathrm{S}\end{array}$ & $\begin{array}{c}23.3 \\
2.7\end{array}$ & $\begin{array}{c}20.7 \\
3.4\end{array}$ & 1 & 3.0 & .005 & .153 & $\sqrt{ }$ \\
\hline
\end{tabular}

Tab. 17: Deskriptive und t-Test-statistische Auswertung der Tests zur phonologischen Bewusstheit.

KTR: Kontrollgruppe; LRS: lese-/rechtschreibschwache Gruppe; $\sqrt{ }$ : Effekt im Sinne der empirischen Vorhersage; durch einen roten

Druck hervorgehoben sind p-Werte, die sich innerhalb der Signifikanzgrenze von $\alpha \leq .05$ befinden.;

e / w : Zugehörigkeit des Test zur phonologischen Bewusstheit im engeren / weteren Sinne. 
In vollständiger Konsistenz mit der empirischen Vorhersage eV 1.b zeigte sich bei jedem Test ein signifikanter erwartungsgemäßer Unterschied zwischen den Experimentalgruppen. Die phonologische Bewusstheit im engeren sowie im weiteren Sinne zeigte sich damit als bei lese/rechtschreibschwachen Probanden im Vergleich zu Kontrollprobanden eingeschränkt.

\subsubsection{ERGEBNISSE DER TESTS ZUM ABRUF AUS DEM LANGZEIT- GEDÄCHTNIS}

Tabelle 18 stellt die Ergebnisse und die statistische Auswertung (t-Tests; Signifikanzniveau: $\alpha=$ .05) des Wortflüssigkeitstests inklusive seiner Untergruppen und des Farben Benennens dar. Sie zeigt, dass beide Tests sowie die Untertests des Wortflüssigkeitstests signifikante Gruppenunterschiede abbildeten, wie es durch die empirische Vorhersage 1.1 formuliert worden war.

\begin{tabular}{|l|l|c|c|c|c|c|c|c|}
\hline & KTR & LRS & $\mathbf{d f}$ & $\mathbf{t}$ & $\mathbf{p}$ & $\eta^{\mathbf{2}}$ & gemäß eV 1.1? \\
\hline WFL- & $\mathrm{M}[\mathrm{N}$ Worte $]$ & $\mathbf{9 . 4}$ & $\mathbf{6 . 9}$ & 1 & 3.7 & .001 & .219 & $\sqrt{ }$ \\
Buchstabe L & $\mathrm{S}$ & 2.2 & 2.6 & & & & & \\
\hline WFL- & $\mathrm{M}[\mathrm{N}$ Worte $]$ & $\mathbf{7 . 6}$ & $\mathbf{5 . 7}$ & 1 & 2.8 & .008 & .136 & $\sqrt{ }$ \\
Buchstabe P & $\mathrm{S}$ & 2.0 & 2.6 & & & & & $\sqrt{ }$ \\
\hline WFL- & $\mathrm{M}[\mathrm{N}$ Worte $]$ & $\mathbf{8 . 2}$ & $\mathbf{6 . 3}$ & 1 & 2.8 & .008 & .136 & \\
Buchstabe R & $\mathrm{S}$ & 2.5 & 2.5 & & & & & $\sqrt{ }$ \\
\hline WFL- & $\mathrm{M}[\mathrm{N}$ Worte $]$ & $\mathbf{2 5 . 6}$ & $\mathbf{1 8 . 9}$ & 1 & 3.9 & .000 & .235 & \\
Gesamt & $\mathrm{S}$ & 5.7 & 6.6 & & & & & $\sqrt{ }$ \\
\hline Farbstriche Be- & $\mathrm{M}[$ sek $]$ & $\mathbf{6 7 . 9}$ & $\mathbf{8 2 . 2}$ & 1 & 2.1 & .044 & .080 & \\
nennen (FSB) & $\mathrm{S}$ & 17.5 & 30.0 & & & & & $\sqrt{ }$ \\
\hline
\end{tabular}

Tab. 18: Deskriptive und t-Test-statistische Auswertung der Tests zum Abruf aus dem Langzeitgedächtnis.

KTR: Kontrollgruppe; LRS: lese-/rechtschreibschwache Gruppe; WFL: Wortflüssigkeitstest;: V: Effekt im Sinne der empirischen Vorhersage; durch einen roten Druck hervorgehoben sind p-Werte, die sich innerhalb der Signifikanzgrenze von $\alpha \leq .05$ befinden.;

\subsubsection{BEURTEILUNG DER EMPIRISCHEN VORHERSAGE 1.0}

Jeder durchgeführte Test führte zu effektstarken erwartungskonformen Gruppenunterschieden. Alle empirischen Vorhersagen (eV 1.a., eV 1.b., eV 1.1) konnten bestätigt werden. Damit kann die übergeordnete empirische Vorhersage 1.0, gemäß der lese-/rechtschreibschwache Proban- 
den im Vergleich zu Kontrollprobanden Beeinträchtigungen der phonologischen Verarbeitung aufweisen, ebenfalls angenommen werden. 


\subsection{GRUPPENUNTERSCHIEDE DER AUDITIV SENSORI- SCHEN VERARBEITUNG}

In einem ersten Abschnitt des Berichts der Ergebnisse zur auditiv sensorischen Verarbeitung steht die Beschreibung der Mismatch Negativity Potenziale im Vordergrund. Aus dem Potenzialverlauf bzw. aus der Segmentierbarkeit der Kurven geht schließlich die Anzahl der Variablen hervor, die für die Berechnungen zur Verfügung stehen. In einem zweiten Abschnitt werden diese Variablen gemäß der empirischen Vorhersage 2.0 auf Gruppenunterschiede hin geprüft. Laut dieser Vorhersage treten Leistungsbeeinträchtigungen lese-/rechtschreibschwacher Probanden im Vergleich zu Kontrollprobanden in den MMN-Variablen auf. Erst im Anschluss an diese Prüfung richtet sich der Blick auf die Untersuchung der Resultate im Sinne der empirischen Vorhersagen 2.s, 2.g und 2.t, um aus der hier erzielten Datenlage einen Hinweis über die Richtigkeit einer der drei konkurrierenden Hypothesen bezüglich eines auditiv sensorischen Verarbeitungsdefizits abzuleiten (spezifisch sprachliches Defizit; generell auditives Defizit; Defizit der rasch temporalen Verarbeitung).

\subsubsection{DESKRIPTION DER POTENZIALE}

Die folgende Abbildung zeigt die erhaltenen Mismatch Negativity-Potenziale sowie die Average-Potenziale des Standard- und des Deviantreizes, aus welchen sich die MMNs berechneten. Mit Ausnahme des Potenzials der dynamischen Sinus-Bedingung konnten alle MMN-Kurven in Teilabschnitte segmentiert werden. In der Abbildung kennzeichnen die Buchstaben A, B oder C die Teilsegmente eines Potenzials. 


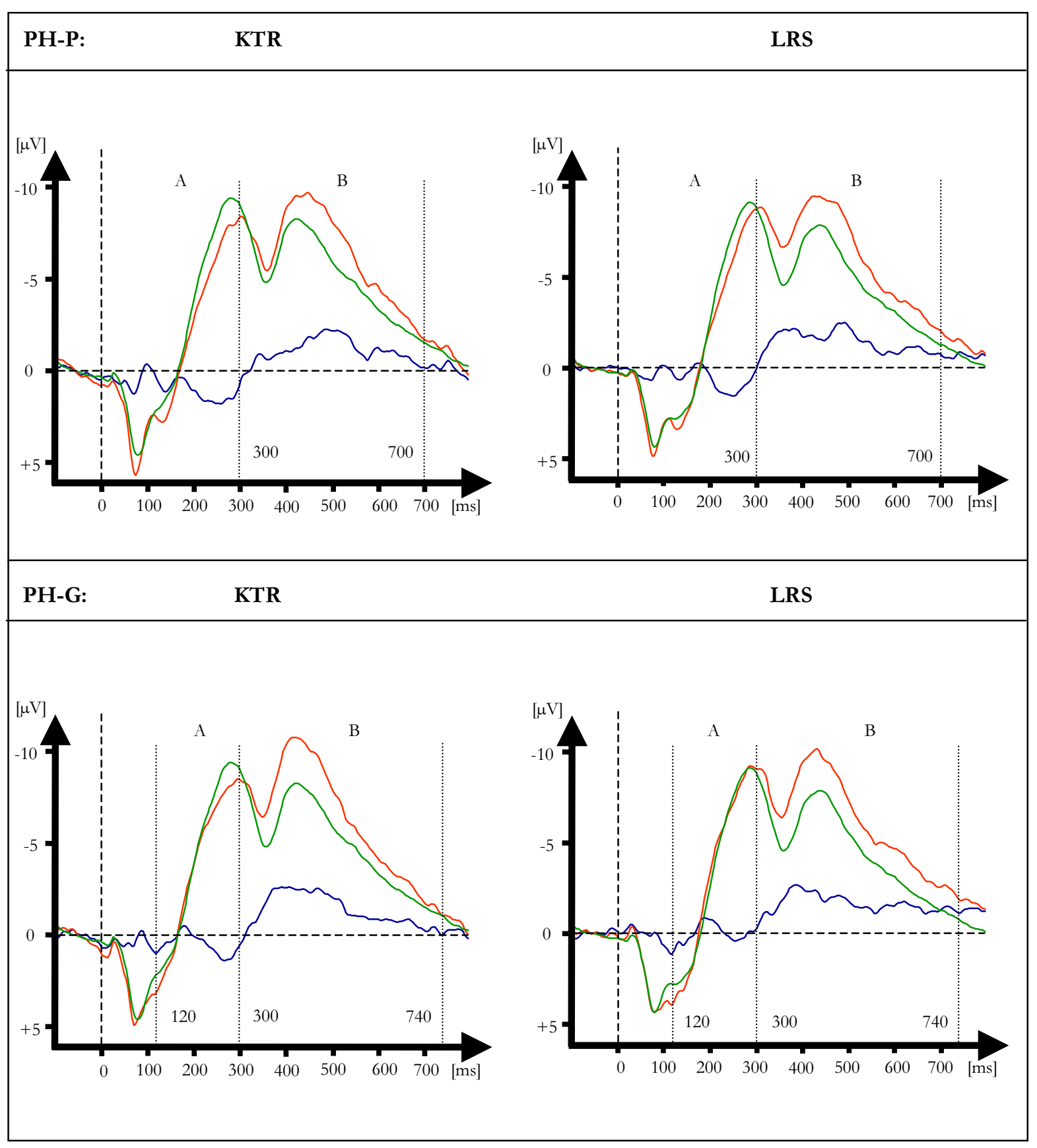




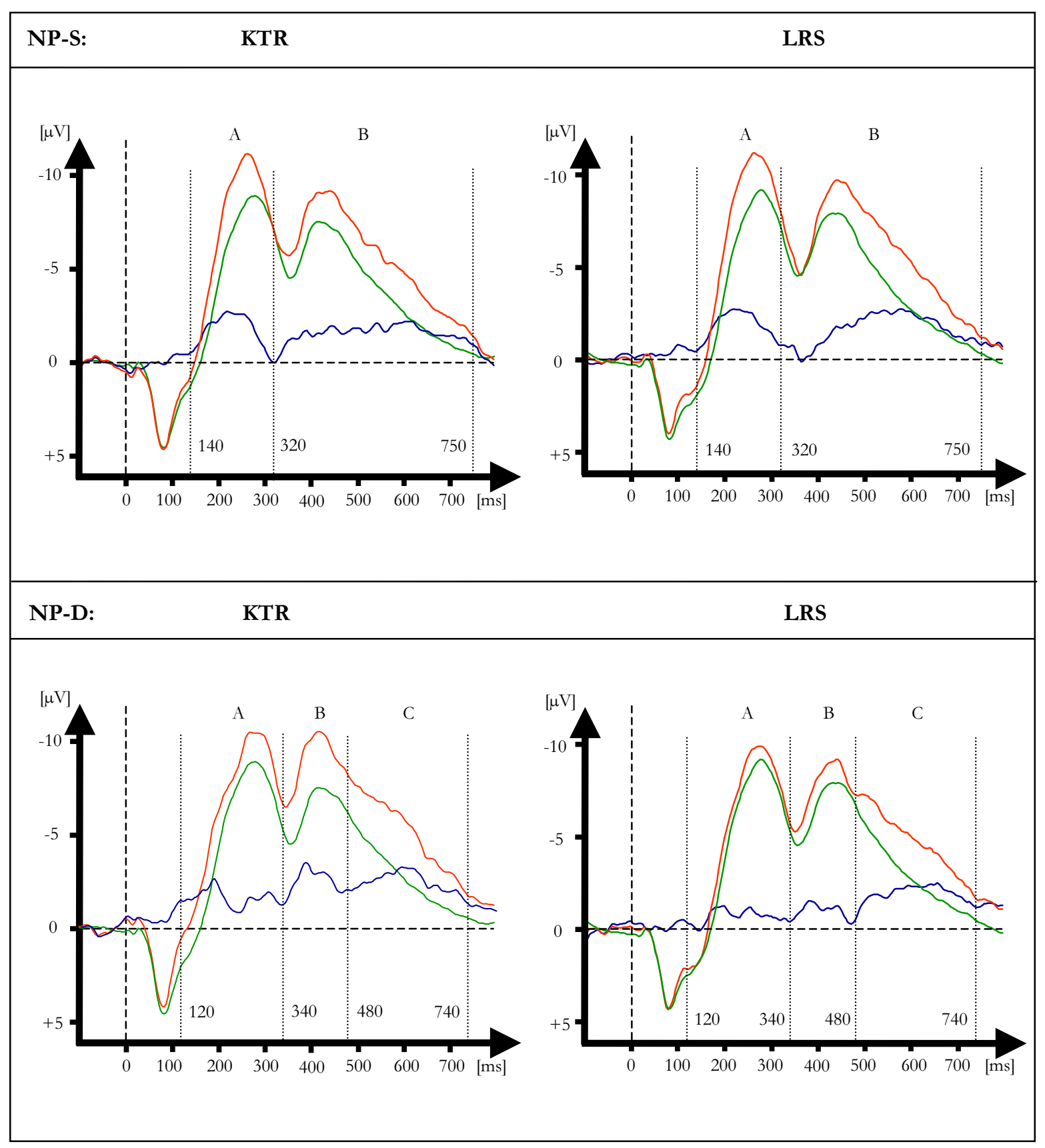




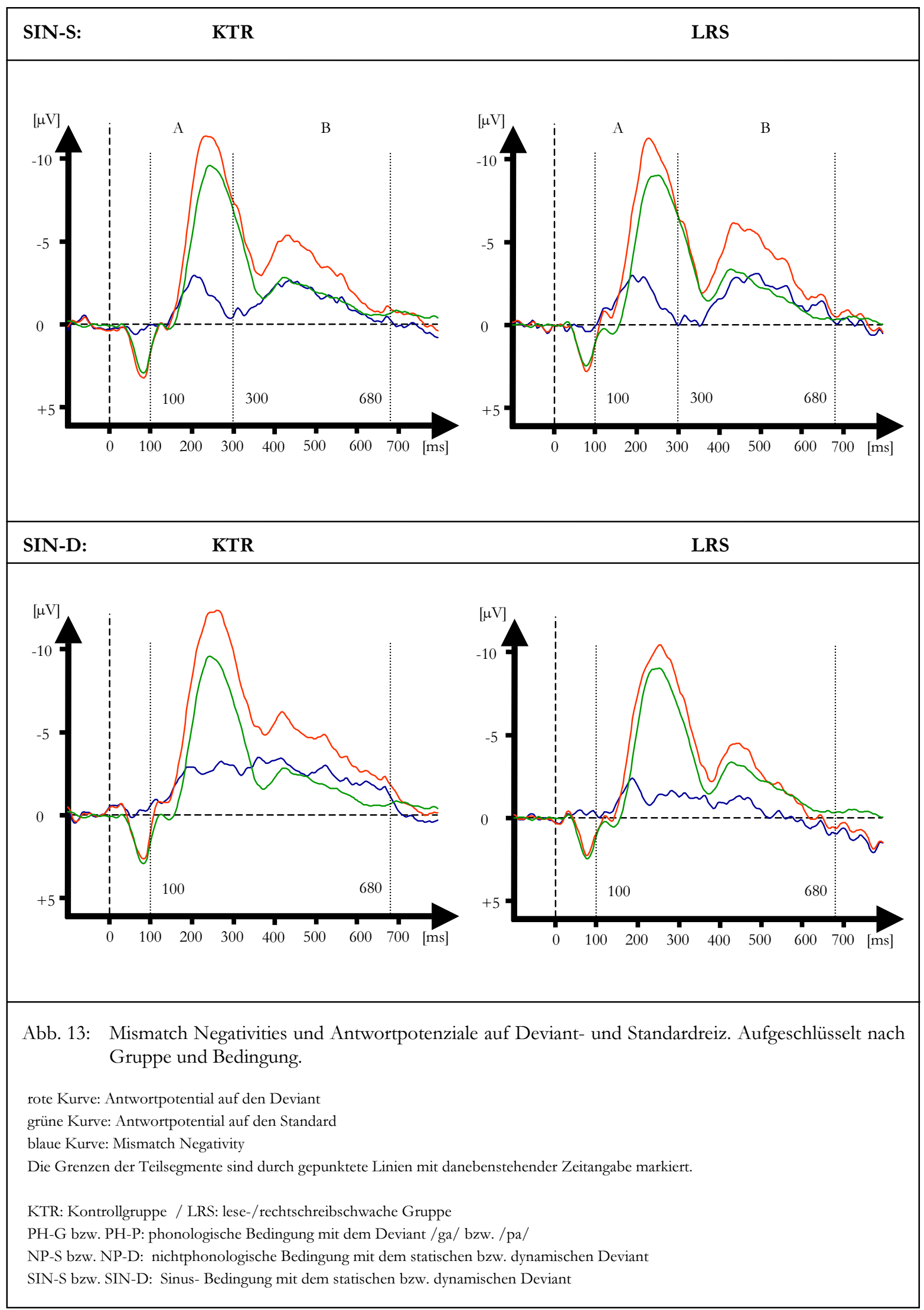


Aus Abbildung 13 ist zu lesen, dass in jeder Bedingung bei jeder Gruppe eine MMN abzuleiten war.

Die Antwortpotenziale auf Standard und Deviant nahmen in jedem Fall einen zweigipfeligen Verlauf. In den Sinus-Bedingungen lag der zweite Gipfel niedriger als der erste; in allen anderen Bedingungen erreichten beide peaks in etwa dieselbe Höhe. Dies ist zu erwarten, da davon auszugehen ist, dass für die Form der Antwortpotenziale vor allem die Träger des Reizunterschiedes verantwortlich sind, welcher in den Sinus-Bedingungen ein Sinuston und in allen anderen Bedingungen die Silbe /ba/ ist.

In der Differenzkurve müsste sich der Unterschied zwischen Deviant und Standard manifestieren: Beide phonologischen Kontrastbedingungen erzeugten eine Mismatch Negativity mit positivem Vorlauf und erst relativ spätem (bei ca. $300 \mathrm{~ms}$ ) Einsatz der (dann eingipfeligen) Negativität. Die Mismatch Negativity beider statischer Deviant-Bedingungen (NP-S, SIN-S) nahm einen klar zweigipfeligen Verlauf, wobei der erste Gipfel früh einsetzte und in etwa halb so lange dauerte (ca. 100 bis $300 \mathrm{~ms}$ bzw. 140 bis $320 \mathrm{~ms}$ ) wie der zweite (ca. 300 bis $680 \mathrm{~ms}$ bzw. 320 bis 750 ms). Der Verlauf der Mismatch Negativity der beiden dynamischen DeviantBedingungen (NP-D, SIN-D) war bezüglich der Herausbildung von Abschnitten uneindeutiger, da das Deviant-Potenzial auf der gesamten Strecke stärker als das Standard-Potenzial war. Die Variation im Ausmaß der stärkeren Intensität der Deviant-Antwort erlaubte bei NP-D eine Segmentierung in (drei) Abschnitte, was bei SIN-D nicht der Fall war. Obwohl die Segmentierung bei SIN-D nicht gerechtfertigt ist, lässt sie sich dort zumindest optisch nachvollziehen. Aus diesem Grunde kann auch bei der Mismatch Negativity der dynamischen Deviants von Ähnlichkeit gesprochen werden. Somit findet sich in den vorliegenden Mismatch NegativityKurven die abschnittseinleitende Behauptung bestätigt, wodurch sie den Kurven Augenscheinvalidität verleiht: Die Ähnlichkeit unter den MMN-Potenzialen ist nicht bei denselben Grundreizen, sondern bei Kontrastierungen ähnlicher Reizqualitäten am größten.

Da für jeden Potenzialabschnitt ein Flächenwert und die Peaklatenz berechnet wurde, standen für die weitere Berechnung insgesamt 24 Variablen zur Verfügung. 


\subsubsection{GRUPPENUNTERSCHIEDE BEI MMN-VARIABLEN}

Mit dem Auge sind an den Kurvenverläufen der Mismatch Negativities nur wenig Gruppenunterschiede auszumachen. Die statistische Überprüfung erfolgte anhand von t-Tests der Flächen- sowie Peaklatenzwerte der MMN-Teilabschnitte. Da sich das MMN-Paradigma -im Gegensatz zu den phonologischen Tests- noch stärker im Etablierungsprozess befindet, wurde eine vorsichtigere Signifikanzgrenze von $\alpha=.10$ festgelegt.

Die Ergebnisse stellt folgende Tabelle dar.

\begin{tabular}{|c|c|c|c|c|c|c|c|c|}
\hline \multicolumn{2}{|c|}{$\begin{array}{l}\text { PHONOLOGISCHE } \\
\text { BEDINGUNG }\end{array}$} & KTR & LRS & df & $\mathbf{t}$ & $\mathrm{p}$ & $\eta^{2}$ & gemäß eV 2.0? \\
\hline \multirow[t]{2}{*}{$\begin{array}{l}\text { PH-P } \\
\text { - Segment A }\end{array}$} & $\begin{array}{l}\mathrm{M}_{\mathrm{A}}[\mu V \times m s] \\
\mathrm{S}\end{array}$ & $\begin{array}{l}1.15 \\
1.75\end{array}$ & $\begin{array}{l}.82 \\
1.67\end{array}$ & 1 & .7 & .503 & .010 & - \\
\hline & $\begin{array}{l}\mathrm{M}_{\mathrm{L}}[m s] \\
\mathrm{S}\end{array}$ & $\begin{array}{c}186 \\
54\end{array}$ & $\begin{array}{c}209 \\
58\end{array}$ & 1 & 1.4 & .167 & .040 & - \\
\hline \multirow[t]{2}{*}{$\begin{array}{l}\text { PH-P } \\
\text { - Segment B }\end{array}$} & $\begin{array}{l}\mathrm{M}_{\mathrm{A}}[\mu V \times m s] \\
\mathrm{S}\end{array}$ & $\begin{array}{l}-1.10 \\
2.24\end{array}$ & $\begin{array}{c}-1.27 \\
2.21\end{array}$ & 1 & .3 & .795 & .001 & - \\
\hline & $\begin{array}{l}\mathrm{M}_{\mathrm{L}}[m s] \\
\mathrm{S}\end{array}$ & $\begin{array}{c}483 \\
93\end{array}$ & $\begin{array}{c}450 \\
95\end{array}$ & 1 & 1.2 & .228 & .031 & - \\
\hline \multirow[t]{2}{*}{$\begin{array}{l}\text { PH-G } \\
\text { - Segment A }\end{array}$} & $\begin{array}{l}\mathrm{M}_{\mathrm{A}}[\mu V \times m s] \\
\mathrm{S}\end{array}$ & $\begin{array}{l}.48 \\
1.85\end{array}$ & $\begin{array}{l}.02 \\
1.39\end{array}$ & 1 & .9 & .339 & .019 & - \\
\hline & $\begin{array}{l}\mathrm{M}_{\mathrm{L}}[m s] \\
\mathrm{S}\end{array}$ & $\begin{array}{c}184 \\
57\end{array}$ & $\begin{array}{c}213 \\
46\end{array}$ & 1 & 1.9 & .057 & .075 & - \\
\hline \multirow[t]{2}{*}{$\begin{array}{l}\text { PH-G } \\
\text { - Segment B }\end{array}$} & $\begin{array}{l}\mathrm{M}_{\mathrm{A}}[\mu V \times m s] \\
\mathrm{S}\end{array}$ & $\begin{array}{c}-1.49 \\
2.44\end{array}$ & $\begin{array}{c}-1.39 \\
1.90\end{array}$ & 1 & 0 & .876 & .001 & - \\
\hline & $\begin{array}{l}\mathrm{M}_{\mathrm{L}}[m s] \\
\mathrm{S}\end{array}$ & $\begin{array}{c}449 \\
96\end{array}$ & $\begin{array}{l}513 \\
117\end{array}$ & 1 & 2.1 & .041 & .086 & $\sqrt{ }$ \\
\hline
\end{tabular}




\begin{tabular}{|c|c|c|c|c|c|c|c|c|}
\hline \multicolumn{2}{|c|}{$\begin{array}{l}\text { NICHTPHONOLOGISCHE } \\
\text { BEDINGUNG }\end{array}$} & \multirow{2}{*}{$\begin{array}{c}\text { KTR } \\
-1.79 \\
2.96 \\
\end{array}$} & \multirow{2}{*}{ 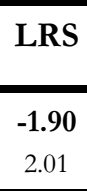 } & \multirow{2}{*}{$\frac{d f}{1}$} & \multirow{2}{*}{$\frac{\mathbf{t}}{0}$} & \multirow{2}{*}{$\begin{array}{c}\mathrm{p} \\
.876\end{array}$} & \multirow{2}{*}{$\frac{\eta^{2}}{.001}$} & \multirow{2}{*}{$\begin{array}{c}\text { gemäß eV 2.0? } \\
-\end{array}$} \\
\hline $\begin{array}{l}\text { NP-S } \\
\text { - Segment A }\end{array}$ & $\begin{array}{l}\mathrm{M}_{\mathrm{A}}[\mu V \times m s] \\
\mathrm{S}\end{array}$ & & & & & & & \\
\hline & $\begin{array}{l}\mathrm{M}_{\mathrm{L}}[m s] \\
\mathrm{S}\end{array}$ & $\begin{array}{c}233 \\
41\end{array}$ & $\begin{array}{c}234 \\
46\end{array}$ & 1 & 0 & .955 & .000 & - \\
\hline \multirow[t]{2}{*}{$\begin{array}{l}\text { NP-S } \\
\text { - Segment B }\end{array}$} & $\begin{array}{l}\mathrm{M}_{\mathrm{A}}[\mu V \times m s] \\
\mathrm{S}\end{array}$ & $\begin{array}{c}-1.58 \\
2.91\end{array}$ & $\begin{array}{c}-1.62 \\
3.17\end{array}$ & 1 & 0 & .959 & .000 & - \\
\hline & $\begin{array}{l}\mathrm{M}_{\mathrm{L}}[m s] \\
\mathrm{S}\end{array}$ & $\begin{array}{l}573 \\
135 \\
\end{array}$ & $\begin{array}{l}516 \\
106\end{array}$ & 1 & 1.6 & .108 & .054 & - \\
\hline \multirow[t]{2}{*}{$\begin{array}{l}\text { NP-D } \\
\text { - Segment A }\end{array}$} & $\begin{array}{l}\mathrm{M}_{\mathrm{A}}[\mu V \times m s] \\
\mathrm{S}\end{array}$ & $\begin{array}{c}-1.64 \\
1.82\end{array}$ & $\begin{array}{l}-.73 \\
2.16\end{array}$ & 1 & 1.6 & .118 & .051 & - \\
\hline & $\begin{array}{l}\mathrm{M}_{\mathrm{L}}[m s] \\
\mathrm{S}\end{array}$ & $\begin{array}{c}220 \\
73\end{array}$ & $\begin{array}{c}229 \\
59\end{array}$ & 1 & .5 & .615 & .005 & - \\
\hline \multirow[t]{2}{*}{$\begin{array}{l}\text { NP-D } \\
\text { - Segment B }\end{array}$} & $\begin{array}{l}\mathrm{M}_{\mathrm{A}}[\mu V \times m s] \\
\mathrm{S}\end{array}$ & $\begin{array}{c}-2.58 \\
2.65\end{array}$ & $\begin{array}{c}-1.04 \\
2.73\end{array}$ & 1 & 2.0 & .051 & .079 & $\sqrt{ }$ \\
\hline & $\begin{array}{l}\mathrm{M}_{\mathrm{L}}[m s] \\
\mathrm{S}\end{array}$ & $\begin{array}{c}408 \\
41\end{array}$ & $\begin{array}{c}410 \\
45\end{array}$ & 1 & 0 & .893 & .000 & - \\
\hline \multirow[t]{2}{*}{$\begin{array}{l}\text { NP-D } \\
\text { - Segment C }\end{array}$} & $\begin{array}{l}\mathrm{M}_{\mathrm{A}}[\mu V \times m s] \\
\mathrm{S}\end{array}$ & $\begin{array}{c}-2.48 \\
3.31\end{array}$ & $\begin{array}{c}-1.85 \\
3.17\end{array}$ & 1 & .7 & .500 & .010 & - \\
\hline & $\begin{array}{l}\mathrm{M}_{\mathrm{L}}[m s] \\
\mathrm{S}\end{array}$ & $\begin{array}{c}607 \\
76\end{array}$ & $\begin{array}{c}596 \\
76\end{array}$ & 1 & .5 & .611 & .006 & - \\
\hline
\end{tabular}

\begin{tabular}{|c|c|c|c|c|c|c|c|c|}
\hline \multicolumn{2}{|c|}{ SINUS - BEDINGUNG } & KTR & LRS & df & $\mathbf{t}$ & $\mathrm{p}$ & $\eta^{2}$ & gemäß eV 2.0? \\
\hline \multirow[t]{2}{*}{$\begin{array}{l}\text { SIN-S } \\
\text { - Segment A }\end{array}$} & $\begin{array}{l}\mathrm{M}_{\mathrm{A}}[\mu V \times m s] \\
\mathrm{S}\end{array}$ & $\begin{array}{c}-1.24 \\
2.60\end{array}$ & $\begin{array}{c}-\mathbf{1 . 8 5} \\
2.39\end{array}$ & 1 & .8 & .398 & .016 & - \\
\hline & $\begin{array}{l}\mathrm{M}_{\mathrm{L}}[m s] \\
\mathrm{S}\end{array}$ & $\begin{array}{c}214 \\
41\end{array}$ & $\begin{array}{c}207 \\
39\end{array}$ & 1 & .6 & .545 & .008 & - \\
\hline \multirow[t]{2}{*}{$\begin{array}{l}\text { SIN-S } \\
\text { - Segment B }\end{array}$} & $\begin{array}{l}\mathrm{M}_{\mathrm{A}}[\mu V \times m s] \\
\mathrm{S}\end{array}$ & $\begin{array}{c}-1.39 \\
3.51\end{array}$ & $\begin{array}{l}-2.13 \\
2.98\end{array}$ & 1 & .8 & .436 & .013 & - \\
\hline & $\begin{array}{l}\mathrm{M}_{\mathrm{L}}[m s] \\
\mathrm{S}\end{array}$ & $\begin{array}{l}440 \\
100\end{array}$ & $\begin{array}{c}469 \\
74\end{array}$ & 1 & 1.1 & .265 & .027 & - \\
\hline \multirow[t]{2}{*}{$\begin{array}{l}\text { SIN-D } \\
\text { - Gesamt }\end{array}$} & $\begin{array}{l}\mathrm{M}_{\mathrm{A}}[\mu V \times m s] \\
\mathrm{S}\end{array}$ & $\begin{array}{c}-2.43 \\
3.09\end{array}$ & $\begin{array}{l}-.91 \\
2.63\end{array}$ & 1 & 1.8 & .074 & .068 & $\sqrt{ }$ \\
\hline & $\begin{array}{l}\mathrm{M}_{\mathrm{L}}[m s] \\
\mathrm{S}\end{array}$ & $\begin{array}{l}374 \\
135\end{array}$ & $\begin{array}{l}386 \\
131\end{array}$ & 1 & .3 & .761 & .002 & - \\
\hline
\end{tabular}

Tab. 19: Deskriptive und t-Test-statistische Auswertung des MMN-Paradigmas.

Durch einen fetten und roten / blauen Druck hervorgehoben sind p-Werte, die sich innerhalb der Signifikanzgrenze von $\alpha \leq .05 /$ $\alpha \leq .10$ befinden.

KTR: Kontrollgruppe; LRS: lese-/rechtschreibschwache Gruppe

PH-P / PH-G: phonologische Bedingung mit den Deviants /pa/ bzw. /ga/; NP-S / NP-D: nichtphonologische Bedingung mit statischem / dynamischem Deviant; SIN-S / SIN-D: Sinus-Bedingung mit statischem / dynamischem Deviant $\mathrm{M}_{\mathrm{A}}$ : Mittelwert der MMN-Fläche; $\mathrm{M}_{\mathrm{L}}$ : Mittelwert der Peaklatenz

$\sqrt{ } /$ - : Effekt / kein Effekt im Sinne der empirischen Vorhersage. 
Der Blick auf die obige Tabelle bestätigt den optischen Eindruck: Das Gesamtbild der neurophysiologischen Untersuchungsreihe zeichnet sich durch eine weitaus geringere Eindeutigkeit im Vergleich zu den Tests der phonologischen Verarbeitung aus. Nur wenige Variablen brachten erwartungskonforme Gruppenunterschiede zutage, die zudem nur geringe Effektstärken aufweisen - die $\eta^{2}$-Werte rangierten zwischen .068 (t-Wert: 1.8) und .086 (t-Wert: 2.1).

Vorhersagekonforme signifikante Gruppenunterschiede traten bei vier Variablen auf. Es handelte sich dabei um verlängerte Latenzen lese-/rechtschreibschwacher Probanden beim ersten und beim zweiten Segment der Bedingung PH-G, geringere negative Flächenwerte beim zweiten Segment des dynamischen nichtphonologischen Bedingung NP-D und eine geringere Fläche beim Gesamtwert der dynamischen Sinus-Bedingung (SIN-D).

Es bleibt zu erwähnen, dass die Gruppenvergleiche aller anderer Variablen insignifikant ausfielen, was impliziert, dass es nicht zu bedeutsamen vorhersagekonträren Gruppenunterschieden kam.

\subsubsection{BEURTEILUNG DER EMPIRISCHEN VORHERSAGE 2.0}

Das Potenzial des ersten Segments der Bedingung PH-G trug zu diesem frühen Zeitpunkt kein negatives, sondern ein positives Vorzeichen. Eine skalpübergreifende topographische Analyse der frontozentralen Aktivität bestätigte dies. Insofern war das ermittelte Differenzpotenzial, dessen Peaklatenz einen gruppenabhängigen signifikanten Unterschied produzierte, nicht als Mismatch Negativity zu erachten.

Die Peaklatenz des zweiten PH-G-Segments bezog sich auf eine negative Flächendifferenz und wurde -ebenso wie die gruppenspezifisch signifikant unterschiedlichen MMN-Flächen der Bedingungen NP-D und SIN-D- auch durch in der topographischen Analyse sichtbare negative Aktivität begleitet. Deren Identität als MMN-Potenziale konnte somit bestätigt werden.

Die statistische Prüfung war hiermit für die Mehrzahl der MMN-Variablen nicht im Sinne der empirischen Vorhersage 2.0 ausgegangen. Da jedoch mit drei erwartungskonformen von insgesamt 24 zur Verfügung stehenden Variablen (12.5\%) das Kriterium zufällig signifikanter Testausgänge von $5 \%$ überschritten wurde, wurde die empirische Vorhersage dennoch als bestätigt angesehen.

Die betreffenden drei Variablen werden, da sie aus verschiedenen Bedingungen stammen, im weiteren Textverlauf vereinfacht mit „PHO”, „NP" und „SIN“ bezeichnet. 


\subsubsection{ZUR SPEZIFIKATION DES AUDITIVEN SENSORISCHEN DEFIZITS}

Drei MMN-Variablen hatten signifikante Gruppenunterschiede erzeugt. Es handelte sich dabei um die Peak-Latenz der MMN des phonologischen Kontrastes mit dem Deviant /ga/, um die MMN-Fläche der MMN der nichtphonologischen Bedingung mit dem dynamischen Deviant und um die MMN-Fläche der MMN der Sinus-Bedingung mit dem dynamischen Deviant.

Fügt man das Muster aus diesen positiven und den negativen Ergebnissen schematisch in die Tabelle ein, die unter dem Punkt „Empirische Vorhersagen“ aufgeführt wurde, entsteht folgendes Bild:

\begin{tabular}{|c|c|c|c|c|c|c|}
\hline \multicolumn{3}{|c|}{ auditiv sensorisches Verarbeitungsdefizit:: } & generell auditiv & spezifisch sprach- & rasch temporal & ERGEBNIS \\
\hline \multirow{4}{*}{$\begin{array}{l}\text { phono- } \\
\text { logischer } \\
\text { Standard }\end{array}$} & \multirow{2}{*}{$\begin{array}{l}\text { phonologische } \\
\text { Kontraste }\end{array}$} & PH-P & $\downarrow$ & $\downarrow$ & $\downarrow /=$ & $=$ \\
\hline & & PH-G & $\downarrow$ & $\downarrow$ & $\downarrow /=$ & $\downarrow$ \\
\hline & \multirow{2}{*}{$\begin{array}{c}\text { nicht-phonologische } \\
\text { Kontraste } \\
\text { (Frequenz) }\end{array}$} & statisch [NP-S] & $\downarrow$ & $=$ & $=$ & $=$ \\
\hline & & dynamisch [NP-D] & $\downarrow$ & $=$ & $\downarrow$ & $\downarrow$ \\
\hline \multirow{2}{*}{$\begin{array}{l}\text { Sinuston- } \\
\text { Standard }\end{array}$} & \multirow{2}{*}{$\begin{array}{c}\text { nicht-phonologische } \\
\text { Kontraste } \\
\text { (Frequenz) }\end{array}$} & statisch [SIN-S] & $\downarrow$ & $=$ & $=$ & $=$ \\
\hline & & dynamisch [SIN-D] & $\downarrow$ & $=$ & $\downarrow$ & $\downarrow$ \\
\hline
\end{tabular}

Tab. 20: Zuordnung des erhaltenen MMN-Bedingungsmusters zu einem Typus auditiv sensorischen Verarbeitungsdefizits.

$=/ \downarrow$ : Die Leistung in der entsprechenden MMN-Bedingung fällt bei lese-/rechtschreibschwachen Probanden genauso gut wie / schlechter als bei Kontrollprobanden aus.

Da die Verarbeitung von Frequenzkontrasten bei lese-/rechtschreibschwachen Kindern sich nicht als beeinträchtigt erwiesen hat, wurde die empirische Vorhersage bezüglich eines generellen auditorischen Defizits (eV 2.g) in dieser Studie nicht bestätigt. Weiterhin ist es wenig wahrscheinlich, dass ein spezifisch sprachliches Defizit vorliegt, wenn nur eine der beiden sprachlichen Bedingungen, aber zwei nichtsprachliche Bedingungen von lese-/rechtschreibschwachen Probanden schlechter passiv diskriminiert wurden. Die empirische Vorhersage 2.s wurde in dieser Studie demzufolge ebenfalls nicht bestätigt. 
Das Muster der Ergebnisse entsprach dem, das für das Vorliegen eines Defizits der rasch temporalen Verarbeitung postuliert wurde, so dass die empirische Vorhersage 2.t angenommen wurde. Kein statischer Frequenzunterschied wurde von lese-/rechtschreibschwachen Kindern schlechter als von Kontrollprobanden verarbeitet, beide dynamischen Frequenzmodulationen deckten jedoch Verarbeitungsschwierigkeiten lese-/rechtschreibschwacher Probanden auf. Da jeweils ein Stimuluspaar phonologischer und damit spektral komplexer Natur war, wird daraus außerdem ersichtlich, dass die Gruppenunterschiede in der passiven Diskrimination des Frequenzunterschieds nicht von der spektralen Komplexität der Stimuli bewirkt worden waren. Die empirische Vorhersage hatte keine signifikanten Gruppenunterschiede in den phonologischen Bedingungen verlangt. Da diese jedoch in der Literatur als die Folge temporal schwacher Verarbeitung postuliert wurden, muss der Befund gleich guter Verarbeitung in einem Fall (PH-P) der beiden phonologischen Bedingungen dennoch wundern. 


\subsection{ZUSAMMENHANG ZWISCHEN PHONOLOGISCHER UND AUDITIV SENSORISCHER VERARBEITUNG}

Insbesondere im Rahmen des Leistungsvergleichs bei kognitionspsychologischen Konstrukten, aber auch im Rahmen des MMN-Paradigmas, wurden Tests identifiziert, die die lese-/rechtschreibschwache Gruppe signifikant von der Kontrollgruppe trennten.

Diese Tests repräsentieren Konstrukte bzw. Verarbeitungsebenen und sollen in Punkt 5.3.2. Auskunft über das Erklärungspotenzial der jeweiligen Konstrukte bzw. Ebenen an der Rechtschreibvarianz geben, um die empirische Vorhersage 3a zu beantworten und $\mathrm{zu}$ spezifizieren. In dieser Vorhersage wurde die Existenz eines Zusammenhangs zwischen kognitionspsychologischen und neurophysiologischen Verarbeitungsdefiziten bei lese-/rechtschreibschwachen Probanden behauptet. Die Modellierbarkeit dieses Zusammenhangs im Sinne der empirischen Vorhersage $3 b$ wird im anschließenden Abschnitt (Punkt 5.3.3.) untersucht.

Bevor es jedoch möglich wird, einen eventuellen Zusammenhang festzustellen bzw. zu explorieren, in welchem die konstituierenden Elemente Konstrukte / Verarbeitungsebenen sind, ist es zuvor nötig, diese Konstrukte bzw. Verarbeitungsebenen zu definieren: In Punkt 5.3.1. wird daher zunächst eine Auswahl aus der Vielzahl zur Verfügung stehender Variablen getroffen, in welcher die verschiedenen Funktionsanforderungen gleichmäßig erfasst werden, in welcher die aussagekräftigsten Variablen zusammengestellt sind und in welcher sichergestellt ist, dass die ausgewählten Variablen tatsächlich die vorgesehenen Verarbeitungsebenen repräsentieren. Weiterhin galt es, die Parameter in eine für die folgenden Berechnungen geeignete statistische Form zu bringen. Diese Auswahl bildet die Grundlage für eine konstrukt- / ebenenbezogene Aggregation von Variablen für die weiteren Analysen (in Punkt 5.3.1.).

\subsubsection{BEREITSTELLUNG GEEIGNETER VARIABLEN FÜR DIE WEITEREN}

\section{ANALYSEN}

\subsubsection{AUSWAHL DER VARIABLEN}

Um inhaltliche Redundanzen zwischen Testvariablen so weit wie möglich zu vermeiden, soll für die weiterführende Analyse pro Aufgabe eine repräsentative Variable bestimmt werden. Das erforderte bei drei Tests, dem Wortflüssigkeitstest, dem Mottier-Test und dem Kunstwort- 
Nachsprechen eine Auswahl aus einem Agglomerat von Untervariablen. Die Wahl sollte jeweils auf diejenige Variable fallen, bei der Gruppenunterschiede am stärksten zu Buche schlugen - was an einem hohen t-Wert sichtbar wurde - , und deren Verteilung sich zugleich einer Normalverteilung annäherte, was bei den feinsten Untergliederungen (von Mottier und Kunstwort-Nachsprechen) nicht immer, ab der ersten Stufe des Zusammenschlusses von Variablen jedoch stets gewährleistet war. Beim Wortproduktionstest wurde der Gruppenunterschied am stärksten durch den Gesamtwert des Tests erfasst. Im Mottier-Test gerieten lese/rechtschreibschwache Probanden besonders bei der Reproduktion der längersilbigen Kunstworte gegenüber gesunden Altersgenossen ins Hintertreffen. Dieser Effekt wurde in einer Variablen abgebildet, die die vier- bis sechssilbigen Items zusammenfasste.

Dasselbe Phänomen war beim Kunstwort-Nachsprechen zu beobachten, wo demzufolge eine gemeinsame Variable für die Reproduktionsleistung der drei- und viersilbigen Kunstworte geschaffen wurde.

Obwohl damit bereits ein Repräsentant für den Kunstworttest gefunden war, ist der potentiellen Aussagekraft des Tests noch nicht Genüge getan, da der Test zusätzlich zur Anforderung an die Dimension des Behaltensvolumens Anforderungen an die Dimension der Speicherpräzision stellt. In diesem Zusammenhang ist die Reproduktionsleistung zweisilbig verzerrter Items interessant, dass diese Variable einen paradoxen, das heißt einen zugunsten der lese-/rechtschreibschwachen Gruppe gerichteten Gruppenunterschied aufgezeigt hatte. Diese Variable war die Basis des erwarteten signifikanten Interaktionseffektes zwischen der Dimension Verzerrungsgrad und dem Gruppenfaktor. Um diesem offenbar bedeutenden Befund Raum zu geben, wurde eine Variable geschaffen, die den Interaktionseffekt zwischen der Silbenanzahl und dem Verzerrungsgrad abbilden sollte: Von der Leistung bei vier- und zweisilbigen unverzerrten Items wurde die Leistung bei den entsprechenden verzerrten Items subtrahiert. Aus diesen beiden Werten wurde wiederum eine Differenz durch den Abzug der Leistung bei zweisilbigen von der Leistung bei viersilbigen Items gebildet. Nach Addition der Konstante 100 und schließlicher Inversion entstand eine normalverteilte Variable, die mit hohen positiven Werten gesunde Leistungen indizierte, signifikante Gruppenunterschiede anzeigte und als solche für den Fortgang der Analyse nutzbar war.

Die beiden selegierten Variablen für die gruppenspezifische Sensibilität gegenüber der Silbenanzahl aus dem Mottier-Test und aus dem Kunstwort-Nachsprechen messen wahrscheinlich sehr ähnliche Sachverhalte und legen dadurch nahe, wenn -wie oben erwähnt, das Ziel besteht, Redundanzen zu vermeiden-, sie als einen Faktor zusammenzufassen. Faktorbildungen führen jedoch zu Informationsreduktion (auf das Gemeinsame). Da beide Tests Grundpfeiler des hier 
zu realisierenden Konstrukts Arbeitsgedächtnis sind und sie zudem durch die beim KunstworteTest hinzukommende Dimension der Itemverzerrung nicht vollständig dasselbe messen, wurde an dieser Stelle die Entscheidung für den Erhalt des vollen Informationsspektrums zum Preis der Inkaufnahme von Redundanzen getroffen.

In der folgenden Tabelle sind die ausgelesenen bzw. geschaffenen Variablen des Wortproduktionstests, des Mottier-Tests und des Kunstworte-Nachsprechens zur Übersicht (z. T. nochmals) aufgeführt:

\begin{tabular}{|l|l|c|c|c|c|c|c|}
\hline & & KTR & LRS & df & $\mathbf{t}$ & $\mathbf{p}$ & $\eta^{\mathbf{2}}$ \\
\hline WFL & M $[$ N Items $]$ & $\mathbf{2 5 . 6}$ & $\mathbf{1 8 . 8}$ & 1 & 3.9 & .000 & .235 \\
Gesamt & $\mathrm{S}$ & 5.7 & 6.6 & & & & \\
\hline MO46 & M $[$ N Items $]$ (max. 18) & $\mathbf{1 3 . 2}$ & $\mathbf{8 . 4}$ & 1 & 4.9 & .000 & .327 \\
& $\mathrm{~S}$ & 3.2 & 3.7 & & & & \\
\hline KN34 & $\mathrm{M}[$ N Items $]$ (max. 16) & $\mathbf{1 2 . 2}$ & $\mathbf{9 . 2}$ & 1 & 4.4 & .000 & .284 \\
& $\mathrm{~S}$ & 2.1 & 2.7 & & & & \\
\hline KNII & $\mathrm{M}$ & $\mathbf{. 9 9 4 2}$ & $\mathbf{. 9 8 3 2}$ & 1 & 2,9 & .005 & .149 \\
& $\mathrm{~S}$ & .0113 & .0152 & & & & \\
\hline
\end{tabular}

Tab. 21: Deskriptive und t-Test-Statistik der für die weitere Auswertung selegierten Variablen des Wortproduktionstests, des Mottier-Tests und des Kunstwort-Nachsprechens.

Da die p-Werte signifikante $(\alpha \leq .05)$ Gruppenunterschiede anzeigen, sind sie durch einen roten Druck hervorgehoben.

KTR: Kontrollgruppe; LRS: lese-/rechtschreibschwache Gruppe

WFL: Wortflüssigkeitstest; MO46: Mottier-Test: vier- bis sechssilbige Items; KN34, KNII: KunstworteNachsprechen: drei- und viersilbige Items, Interaktionswert.

\subsubsection{VERIFIKATION DER REALISATION DER KONSTRUKTE}

Um sicherzustellen, dass die selegierten $\mathrm{zu}$ einem bestimmten kognitionspsychologischen Konstrukt gehörigen Variablen in der Tat eine Zusammengehörigkeit im Sinne der jeweiligen Konstrukte aufwiesen (phonologische Bewusstheit, phonologisches Arbeitsgedächtnis und der Abruf aus dem Langzeitgedächtnis), wurde eine Faktorenanalyse durchgeführt.

Ladungen der Variablen auch auf den Faktoren anderer Konstrukte galten nicht als Falsifikationsargument, da aufgrund der inhaltlichen Nähe der Konstrukte hiervon auszugehen war. Variablen, die jedoch auf dem ihnen zugehörigen Faktor keine Ladung aufwiesen, wurden von 
der weiteren Analyse ausgeschlossen. Dies traf für zwei Variablen zu, die Wortspanne und das Worte Ergänzen des PET (vgl. Tab. 24).

Um sich eine Orientierung zu verschaffen, ob auch die EEG-Variablen eine Ebene bilden würden und wo diese im Dimensionsraum der kognitionspsychologischen Konstrukte sein würde, wurden auch die EEG-Variablen in die Faktorenanalyse eingeschlossen. Da die MMNVariablen jedoch weniger aufgrund inhaltlicher, sondern vor allem durch äußerliche experimentelle Gegebenheiten geeint werden, bestand für sie keine Restriktion hinsichtlich ihrer Lage bzw. der Anzahl der Dimensionen.

Für die Bestimmung der Anzahl der fünf zu extrahierenden Faktoren wurde das KaiserKriterium angewandt; die Extraktionsmethode bestand in einer Hauptkomponentenanalyse mit rotierten Faktoren. Eine solche Faktorenanalyse ohne die ausgeschlossenen Variablen liefert folgendes Bild:

\begin{tabular}{|c|c|c|c|c|c|c|}
\hline & & I & II & III & IV & $\mathrm{V}$ \\
\hline 0 & PHO & .066 & .070 & .061 & .829 & .135 \\
\hline 띠 & NP & .120 & .495 & -.135 & -.268 & .462 \\
\hline 되 & SIN & .601 & -.059 & -.090 & -.396 & .311 \\
\hline \multirow{5}{*}{ 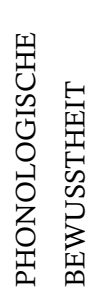 } & PET LV & .523 & .396 & -.105 & .381 & -.335 \\
\hline & WE & .707 & .404 & .171 & .135 & .014 \\
\hline & WT & .719 & .146 & .139 & .169 & .111 \\
\hline & WR & .468 & .457 & .388 & .380 & .096 \\
\hline & WK & .711 & .472 & .108 & -.160 & -.046 \\
\hline \multirow{2}{*}{ 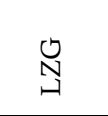 } & WFL & .667 & .221 & .403 & .159 & -.017 \\
\hline & FSB & .276 & -.082 & .858 & .056 & .151 \\
\hline \multirow{6}{*}{ 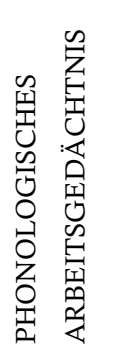 } & KNII & .050 & .122 & .102 & .204 & .841 \\
\hline & KN34 & .205 & .790 & .099 & .041 & .181 \\
\hline & MO46 & .395 & .777 & .008 & .135 & .057 \\
\hline & TRI & .442 & .502 & .273 & -.133 & .026 \\
\hline & $\mathrm{ZR}$ & .018 & .574 & .694 & .050 & -.164 \\
\hline & $\mathrm{ZV}$ & .162 & .648 & .084 & .371 & .026 \\
\hline \multicolumn{7}{|c|}{$\begin{array}{l}\text { Tab 22: Rotierte Faktorladungsmatrix einer Faktorenanalyse (Hauptkomponentenanalyse) } \\
\text { LZG: Abruf aus dem Langzeitgedächtnis; } \\
\text { PHO: Peaklatenz der MMN in PH-G, Segment B; NP: Fläche der MMN in NP-D, Segment B; SIN: Fläche der MMN in } \\
\text { SIN-D. } \\
\text { PET LV: aus PET: Laute verbinden; WE, WT, WR, WK: jew. aus Würzburger Testbatterie: Laute ergänzen, Laute } \\
\text { vertauschen, Restworte, Laute klassifizieren; WFL: Wortflüssigkeitstest; FSB: Farbstriche Benennen; KNII, KN34: } \\
\text { Kunstworte-Nachsprechen: Interaktionsvariable; 3- und 4-silbige Items; MO46: 4- bis 6-silbige Pseudoworte im Mottier- } \\
\text { Test; TRI: Trigramm-Test; ZR, ZV: HAWIK-III: Zahlen Nachsprechen rückwärts bzw. vorwärts. }\end{array}$} \\
\hline
\end{tabular}


Es wurde deutlich, dass die verbleibenden Variablen durchaus Zusammengehörigkeiten im Sinne der Zugehörigkeit zu einem kognitionspsychologischen Konstrukt aufwiesen. Besonders traf dies zu für die phonologische Bewusstheit (Faktor I) und das phonologische Arbeitsgedächtnis (Faktor II) mit Ausnahme der Interaktionsvariable, die trotzdem -wenn auch nur gering- lud. Die Gemeinsamkeit der beiden Variablen des Abrufs aus dem Langzeitgedächtnis ließ sich etwas weniger deutlich, aber dennoch existent, auf Faktor I und Faktor III nachvollziehen. Auf Faktor III fand sich weiterhin das Zahlen Nachsprechen in umgekehrter Reihenfolge mit hoher Ladung. Dies war vermutlich als Ausdruck der exekutiven Funktionen zu verstehen, die mit diesem Test wie auch mit dem Stroop-Test untersucht werden können, hier jedoch nicht thematisiert werden.

Die Interaktionsvariable des Kunstwort-Nachsprechens lud ohne eine weitere bedeutende kognitionspsychologische Coladung auf dem fünften Faktor. Allerdings bestand über die MMNVariable NP eine Verbindung zum Arbeitsgedächtnis-Faktor, da diese auf den beiden Faktoren (mit derselben Intensität) lud. Möglicherweise deutet die abseits stehende, aber mit dem Arbeitsgedächtnis-Faktor verbundene Variable auf ein zum Arbeitsgedächtnis gehöriges Merkmal hin, das von den üblichen Tests bislang nicht erfasst wurde. Da dieses Merkmal offenbar eine starke Gemeinsamkeit mit einer neurophysiologischen Variable aufweist, ist denkbar, dass es eine frühere im Sinne von physiologischere Ebene der Verarbeitung repräsentiert.

Für die MMN-Variablen zeichnete sich ab, dass sie nicht als Ausdruck eines einheitlichen Konstrukts zu verstehen sein würden, da jede auf anderen Faktoren lud. Weiterhin deutete das Muster der Ladungen Beziehungen zu den kognitionspsychologischen Variablen an, die sich jedoch im weiteren Verlauf der Analyse zu bestätigen haben:

Der Sinus-Parameter wies Gemeinsamkeiten mit der phonologischen Bewusstheit und dem Abruf aus dem Langzeitgedächtnis auf; die nichtphonologische MMN schien mit den Arbeitsgedächtnisvariablen zu korrelieren - seien sie auf Faktor II oder Faktor V. Auffällig war die Mismatch Negativity des phonologischen Deviants, die einen Faktor so gut wie alleine beanspruchte. Möglicherweise beinhaltet diese Variable Information, die von den anderen Variablen nicht geliefert wird.

\subsubsection{ADAPTATION VON RichtUNG UND POLUNG}

Die weitere Analyse wird auf korrelativen Verfahren basieren. Es vereinfacht die Interpretation der resultierenden Zusammenhänge, wenn alle eingehenden Parameter dieselbe Polung und 
Richtung besitzen. Demzufolge wurden alle Variablen so eingestellt, dass höhere positive Werte bessere Leistungen indizierten. Die Geschwindigkeitsvariablen (Trigramme, Farben Benennen, Latenz der MMN der phonologischen Bedingung PH-G) wurden zu diesem Zweck invertiert und negative Parameter (Amplituden der MMNs der dynamischen nichtphonologischen und dynamischen Sinus-Bedingung) umgepolt. Bei der Interaktionsvariable des Kunstworttests trat der Wert 0 auf, so dass keine einfache Inversion möglich war; mit ihr wurde verfahren wie oben beschrieben $\left(\{1 /[\text { Interaktionsvariable }+100]\}^{*} 100\right)$.

Jede Transformation erfolgte unter Erhalt der Verteilungsparameter und der Stärke des Gruppenunterschiedes der Ausgangsvariable.

In die obige Faktorenanalyse waren bereits die transformierten Variablen eingegangen.

\subsubsection{ERKLÄRUNGSKRAFT DER AGGREGIERTEN ABHÄNGIGEN VARIAB- LEN FÜR DIE RECHTSCHREIBLEISTUNG}

Die folgenden Abschnitte dienen der Feststellung und der Spezifikation eines Zusammenhangs zwischen kognitionspsychologischer und neurophysiologischer Verarbeitung im Hinblick auf die Rechtschreibleistung. Nach der Festlegung von konstruktspezifischen Variablenaggregationen (in Punkt 5.3.2.1.) ist anhand dieser zu untersuchen, ob und inwieweit Überschneidungen der Verarbeitungsebene / Konstrukte bei der Vorhersage der Rechtschreibleistung bestehen bzw. ob sich von seiten der neurophysiologischen Ebene Vorhersagepotenziale ergeben, die von der kognitionspsychologischen Seite noch nicht erfasst werden. Hierzu wird in einem ersten Schritt (Punkt 5.3.2.2.) eine Struktur der Potenziale der kognitionspsychologischen Konstrukte ermittelt; im folgenden Punkt (5.3.2.3.) wird die Ebene der neurophysiologischen Verarbeitung in diese Struktur implementiert.

\subsubsection{AGgREGATION DER ABHÄNGIGEN VARIABLEN UND BESTIMMUNG DER ERKLÄRUNGSKRAFT DER AGGREGATE}

Um Abhängigkeiten zwischen kognitionspsychologischer und neurophysiologischer Verarbeitungsebene im Zusammenhang mit der Rechtschreibleistung ermitteln und spezifizieren zu können, müssen die Konstrukte / Verarbeitungsebenen definiert werden. Mit dem Erstellen einer Auswahl von Variablen, deren Zugehörigkeit zu einem intendierten Konstrukt bestätigt wurde, wurde ein Aspekt dieser Definition realisiert. 
Möglicherweise treten jedoch innerhalb der Auswahl an Variablen, die zu einem Konstrukt gehören, inhaltliche Redundanzen auf. Es war von daher sinnvoll, einen weiteren selegierenden Schritt zu unternehmen und für jedes Konstrukt ein Agglomerat an Variablen zusammenzustellen, das zwar möglichst nahe an das Erklärungspotenzial des Zusammenschlusses aller zu einem Konstrukt gehörigen Variablen heranreichten, deren Variablen hinsichtlich ihres jeweiligen Erklärungspotenzials dabei aber möglichst wenig Überlappungen aufwiesen.

$\mathrm{Zu}$ diesem Zweck eignete sich die multiple Regression mit ihrem Modul „Stepwise“. Mithilfe der multiplen Regression lässt sich das Erklärungspotenzial (Varianz) einer Gesamtheit von Variablen auf eine Zielvariable berechnen. Auf diese Art konnten demzufolge die Erklärungspotenziale der Konstrukte berechnet werden, wenn alle zur Verfügung stehenden Variablen einbezogen werden.

Das Modul „Stepwise“ als Besonderheit des Verfahrens prüft die Verbesserung der Vorhersage durch sukzessives Hinzuziehen jeder einzelnen aus dem Pool prädiktiver Variablen. Diese Prüfung ist an ein festzulegendes Signifikanzkriterium gebunden, welches hier moderat mit $\alpha=$ .10 bestimmt wurde. Die konstruktspezifischen Agglomerate konstituierten sich damit aus den Variablen, die durch das Stepwise-Verfahren als auf einem Signifikanzniveau von .10 als vorhersageverbessernd eingestuft wurden.

Als Parameter der multiplen Regression wird üblicherweise die korrigierte, d.h. die auf die Population bezogene, Form des Regressionsparameters $\mathrm{R}^{2}$ verwendet. Im weiteren Analyseverlauf werden hier jedoch viele vergleichende Berechnungen notwendig, in denen es auf die Relation von $\mathrm{R}^{2}$-Werten derselben Variable bzw. desselben Konstrukts unter verschiedenen Bedingungen ankommt. Innerhalb dieser Vergleiche gewähren die unkorrigierten Prädiktionskoeffizienten - da sie keinem weiteren Rechenschritt unterzogen wurden - die höhere Konsistenz und werden daher für die Beantwortung der hiesigen Fragestellungen als geeigneter erachtet. Der korrigierte Wert wird jedoch stets mit angegeben.

Tabelle 23 dokumentiert die Gesamtmaße maximal möglicher Regression auf die Rechtschreibleistung, die sich ergeben, wenn alle zu einem Konstrukt gehörigen Variablen in die Vorhersage einbezogen werden, sowie die Auslese der besonders prädiktiven Variablen durch die StepwiseMethode. 


\begin{tabular}{|c|c|c|c|c|c|c|c|c|c|c|}
\hline \multirow{2}{*}{ AG } & \multirow[t]{2}{*}{$\mathbf{R}^{2}$} & \multirow{2}{*}{$\begin{array}{c}\mathbf{R}^{2} \\
\text { korr. }\end{array}$} & \multirow{2}{*}{$\begin{array}{c}\text { SFe d. } \\
\text { Schät- } \\
\text { zers }\end{array}$} & \multirow{2}{*}{$\begin{array}{l}\text { Ände- } \\
\text { rung } R^{2}\end{array}$} & \multirow{2}{*}{$\begin{array}{l}\text { Ände- } \\
\text { rung F }\end{array}$} & \multirow[t]{2}{*}{ df 1} & \multirow[t]{2}{*}{ df 2} & \multirow{2}{*}{$\begin{array}{l}\text { Änderung } \\
\text { F-Sign. }\end{array}$} & \multicolumn{2}{|c|}{ Ausgeschlossen } \\
\hline & & & & & & & & & t-Wert & sign. \\
\hline Gesamt & .623 & .570 & 9.67 & .623 & 11.8 & 6 & 43 & .000 & & \\
\hline step: & & & & & & & & & & \\
\hline MO46 & .485 & .474 & 10.70 & .485 & 45.2 & 1 & 48 & .000 & & \\
\hline TRI & .587 & .570 & 9.68 & .102 & 11.6 & 1 & 47 & .001 & & \\
\hline KNII & .621 & .596 & 9.38 & .034 & 4.1 & 1 & 46 & .049 & & \\
\hline KN34 & & & & & & & & & .37 & .713 \\
\hline ZV & & & & & & & & & .36 & .719 \\
\hline ZR & & & & & & & & & .21 & .833 \\
\hline
\end{tabular}

AG: phonologisches Arbeitsgedächtnis; MO46: vier- bis sechssilbige Items des Mottier; TRI: Trigramme; KNII, KN34: KunstwortNachsprechen: Interaktionswert, drei- bis viersilbige Items; ZV: Zahlen Nachsprechen vorwärts; ZR: Zahlen Nachsprechen rückwärts

\begin{tabular}{|l|c|c|c|c|c|c|c|c|c|c|}
\hline BW & $\mathbf{R}^{2}$ & $\begin{array}{c}\mathbf{R}^{2} \\
\text { korr. }\end{array}$ & $\begin{array}{c}\text { SFe d. } \\
\text { Schät- } \\
\text { zers }\end{array}$ & $\begin{array}{c}\text { Ände- } \\
\text { rung } \mathbf{R}^{2}\end{array}$ & $\begin{array}{c}\text { Ände- } \\
\text { rung F }\end{array}$ & df 1 & df 2 & $\begin{array}{c}\text { Änderung } \\
\text { F-Sign. }\end{array}$ & \multicolumn{2}{|l|}{ Ausgeschlossen } \\
\hline Gesamt & $\mathbf{. 7 1 0}$ & .678 & 8.38 & .710 & 22.0 & 5 & 45 & .000 & & \\
\hline step: & & & & & & & & & & \\
\hline WE & $\mathbf{. 5 5 8}$ & .549 & 9.91 & .558 & 61.9 & 1 & 49 & .000 & & \\
\hline WT & $\mathbf{. 6 6 3}$ & .649 & 8.75 & .105 & 14.9 & 1 & 48 & .000 & & \\
\hline WK & $\mathbf{. 6 9 6}$ & .676 & 8.40 & .033 & 5.1 & 1 & 47 & .028 & & \\
\hline PET LV & & & & & & & & & .22 & .823 \\
\hline WR & & & & & & & & & 1.50 & .140 \\
\hline
\end{tabular}

BW: phonologische Bewusstheit; Würzburger Testbatterie: WE: Laute Ersetzen; WT: Laute Vertauschen; WK: Laute Klassifizieren; WR: Restworte; PET WE: PET: Wörter Ergänzen; PET LV: PET: Laute Verbinden

\begin{tabular}{|l|c|c|c|c|c|c|c|c|c|c|}
\hline LZG & $\mathbf{R}^{2}$ & $\begin{array}{c}\mathbf{R}^{2} \\
\text { korr. }\end{array}$ & $\begin{array}{c}\text { SFe d. } \\
\text { Schät- } \\
\text { zers }\end{array}$ & $\begin{array}{c}\text { Ände- } \\
\text { rung } \mathbf{R}^{2}\end{array}$ & $\begin{array}{c}\text { Ände- } \\
\text { rung F }\end{array}$ & df 1 & df 2 & $\begin{array}{c}\text { Änderung } \\
\text { F-Sign. }\end{array}$ & \multicolumn{2}{|c|}{ Ausgeschlossen } \\
\hline Gesamt & .291 & .262 & 12.68 & .291 & 9.9 & 2 & 48 & .000 & & \\
\hline step: & & & & & & & & & & \\
\hline WFL & .283 & .286 & 12.62 & .283 & 19.4 & 1 & 49 & .000 & & \\
\hline FSB & & & & & & & & & .74 & .462 \\
\hline
\end{tabular}

LZG: Zugriff auf das Langzeitgedächtnis; Wortflüssigkeitstest; FSB: Farbstriche Benennen

\begin{tabular}{|c|c|c|c|c|c|c|c|c|c|c|}
\hline \multirow[t]{2}{*}{ EEG } & \multirow[t]{2}{*}{$\mathbf{R}^{2}$} & \multirow{2}{*}{$\begin{array}{c}\mathbf{R}^{2} \\
\text { korr. }\end{array}$} & \multirow{2}{*}{$\begin{array}{c}\text { SFe d. } \\
\text { Schät- } \\
\text { zers }\end{array}$} & \multirow{2}{*}{$\begin{array}{l}\text { Ände- } \\
\text { rung } R^{2}\end{array}$} & \multirow{2}{*}{$\begin{array}{l}\text { Ände- } \\
\text { rung F }\end{array}$} & \multirow[t]{2}{*}{ df 1} & \multirow[t]{2}{*}{ df 2} & \multirow{2}{*}{$\begin{array}{c}\text { Änderung } \\
\text { F-Sign. }\end{array}$} & \multicolumn{2}{|c|}{ Ausgeschlossen } \\
\hline & & & & & & & & & $t$-Wert & sign. \\
\hline Gesamt & .283 & 234 & 12.91 & .283 & 5.8 & 3 & 44 & .002 & & \\
\hline step: & & & & & & & & & & \\
\hline NP & .117 & .098 & 14.02 & .117 & 6.1 & 1 & 46 & .017 & & \\
\hline SIN & .193 & .157 & 13.55 & .076 & 4.2 & 1 & 45 & .045 & & \\
\hline PHO & .283 & .234 & 12.91 & .090 & 5.5 & 1 & 44 & .023 & & \\
\hline
\end{tabular}

NP: Flächenwert der MMN aus NP-D; Segment B; SIN: Flächenwert der MMN aus SIN-D; PHO: Peaklatenz der MMN aus PH-G, Segment B

Tab. 23:

Regression der Gesamtauswahl der Variablen eines Konstrukts und Regression der mittels der Stepwise-

Technik ausgewählten Variablen auf die Rechtschreibleistung.

Die Zeile „Gesamt“ gibt das Erklärungspotential wieder, das sich durch das Einbeziehen aller pro Ebene vorhandenen Variablen ergibt. Die darunterstehenden Zeilen („step“) beschreiben die Regression beim schrittweisen Hinzuziehen der einzelnen Variablen. In den beiden rechten Spalten finden sich die für den Ausschluss verantwortlichen Werte ausgeschlossener Variablen.

$\mathrm{R}^{2}$ (korr.): (korrigierte) Varianzaufklärung; Schätzung S: Schätzung der Varianz; Änderung F: Änderung des F-Werts; Änderung F-Sign.: Änderung der Signifikanz des F-Werts. 
Tabelle 23 ist zu entnehmen, dass die phonologische Bewusstheit den größten Erklärungsanteil an der Rechtschreibleistung trug $\left(\mathrm{R}^{2}=.710 ; \mathrm{R}^{2}{ }_{\text {korr }}=.678\right)$, der Beitrag des phonologischen Arbeitsgedächtnisses beinahe ebenso groß war $\left(\mathrm{R}^{2}=.623 ; \mathrm{R}^{2}{ }_{\text {korr }}=.570\right)$ und der Abruf aus dem Langzeitgedächtnis nur etwa die Hälfte des Arbeitsgedächtnisanteils ausmachte $\left(\mathrm{R}^{2}=.291\right.$; $\mathrm{R}^{2}{ }_{\text {korr }}=.262$ ). Das vergleichsweise geringe Vorhersagepotenzial des Abrufs aus dem Langzeitgedächtnis mochte dadurch begründet sein, dass dieses Konstrukt nur durch zwei Test konstituiert wurde. Mit einer Regression von $\mathrm{R}^{2}=.283$ bzw. $\mathrm{R}^{2}$ korr $=.234$ befand sich der Varianzanteil der drei EEG-Variablen in der Größenordnung des Abrufs aus dem Langzeitgedächtnis.

Es zeigte sich, dass die Regressionsleistung jeden kognitionspsychologischen Verarbeitungskonstrukts nahezu vollständig durch einen Ausschnitt der zur Verfügung stehenden Variablen erklärt werden konnte. Diese repräsentativen Variablen wurden fürderhin herangezogen, um ein mögliches Zusammenspiels zwischen den Konstrukten / Ebenen zu untersuchen.

Für das Arbeitsgedächtnis erwiesen sich der Mottier-, der Trigramm-Test und die Interaktionsvariable des Kunstwort-Nachsprechens als varianztragende Elemente. Die Gedächtnisspannenvariablen sowie die Speichervariable des Kunstwort-Nachsprechens fielen in diesem Kontext offenbar weniger ins Gewicht. Die Regression der phonologischen Bewusstheit wurde hauptsächlich durch drei Variablen der Würzburger Testbatterie getragen: dem Laute Ersetzen, dem Laute Vertauschen und dem Laute Klassifizieren. Auch die nur zwei Variablen zum Abruf aus dem Langzeitgedächtnis teilten sich in eine hauptvarianzerklärende und in eine weniger erklärungspotente Variable. Die varianztragende Variable war der Wortflüssigkeitstest.

Vor dem Hintergrund, dass die EEG-Variablen bereits in der Faktorenanalyse jeweils ein unterschiedliches Ladungsverhalten gezeigt hatten, ist das Resultat der multiplen StepwiseRegression nicht überraschend: Alle drei Variablen trugen signifikant zur Erklärung der Rechtschreibvarianz bei. 


\subsubsection{STRUKTUR DER VARIANZEN DER KOGNITIONSPSYCHOLOGISCHEN KONSTRUKTE}

Die Varianzanteile der kognitionspsychologischen Verarbeitungskonstrukte sind bei weitem nicht voneinander unabhängig. $\mathrm{Da}$ alle drei Konstrukte Aspekte phonologischer Verarbeitung sind, ist dies theoretisch einsichtig und wurde außerdem von der obigen Faktorenanalyse angedeutet.

Aus einem Blick auf die Regressionswerte der Konstrukte ließ sich dasselbe ablesen: Deren Addition würde $\mathrm{zu}$ dem absurden Wert von $\mathrm{R}^{2}=1.6$ führen. Die Varianzräume der kognitionspsychologischen Konstrukte mussten demzufolge weitreichende Überschneidungen aufweisen. Wo zu lokalisieren und wie stark zu quantifizieren diese Überschneidungen waren, ist der Inhalt des folgenden Abschnitts. Die Überlegungen sollten in die Aufstellung eines Schemas über das Zusammenspiel der kognitionspsychologischen Konstrukte im Varianzraum der Rechtschreibleistung münden. (Der Frage, wie sich die EEG-Variablen in diesen Zusammenhang einfügen, widmet sich der darauffolgende Abschnitt und schließt wiederum mit einem Schema.)

Methodische Grundlage des Vorhabens bildete erneut die Stepwise-Technik der multiplen Regression: Die repräsentativen Variablen eines Konstrukts wurden mit denjenigen eines anderen Konstrukts kombiniert. Variablen, die bei der Endauswahl der Stepwise-Prozedur nicht berücksichtigt wurden, zeigten sich überschneidende Bereiche an; berücksichtigte Variablen deuteten auf Bereiche additiver Varianzerklärung hin. Die gewonnenen Resultate wurden einer Gegenprüfung unterzogen.

Die Tabelle 24 dokumentiert und das folgende Diagramm (Abb. 14) veranschaulicht die Ergebnisse der Kombinationen. 


\begin{tabular}{|c|c|c|c|c|c|c|c|c|c|c|}
\hline \multirow[t]{2}{*}{$A G+B W$} & \multirow[t]{2}{*}{$\mathbf{R}^{2}$} & \multirow{2}{*}{$\begin{array}{c}\mathbf{R}^{2} \\
\text { korr. }\end{array}$} & \multirow{2}{*}{$\begin{array}{c}\text { SFe d. } \\
\text { Schätzers }\end{array}$} & \multirow{2}{*}{\begin{tabular}{|c|} 
Ände- \\
rung $R^{2}$
\end{tabular}} & \multirow{2}{*}{$\begin{array}{l}\text { Ände- } \\
\text { rung F }\end{array}$} & \multirow[t]{2}{*}{ df 1} & \multirow[t]{2}{*}{ df 2} & \multirow{2}{*}{$\begin{array}{c}\text { Änderung } \\
\text { F-Sign. }\end{array}$} & \multicolumn{2}{|c|}{ Ausgeschlossen } \\
\hline & & & & & & & & & t-Wert & sign. \\
\hline Gesamt & .773 & .742 & 7.50 & .773 & 24.9 & 6 & 44 & .000 & & \\
\hline \multicolumn{11}{|l|}{ step: } \\
\hline WE & .558 & .549 & 9.9 & .558 & 61.9 & 1 & 49 & .000 & & \\
\hline WT & .663 & .649 & 8.8 & .105 & 14.9 & 1 & 48 & .000 & & \\
\hline MO46 & .727 & .710 & 8.0 & .064 & 11.1 & 1 & 47 & .002 & & \\
\hline TRI & .751 & .729 & 7.7 & .023 & 4.3 & 1 & 46 & .043 & & \\
\hline KNII & .768 & .742 & 7.5 & .017 & 3.3 & 1 & 45 & .075 & & \\
\hline WK & & & & & & & & & .99 & 328 \\
\hline
\end{tabular}

AG: Arbeitsgedächtnis; BW: phonologische Bewusstheit; MO46: 4- bis 6silbige Items des Mottier; TRI: Trigramme; KNII: Interaktionswert; WK: Laute Klassifizieren.

\begin{tabular}{|c|c|c|c|c|c|c|c|c|c|c|}
\hline \multirow{2}{*}{$\begin{array}{l}\text { AG + } \\
\text { LZG }\end{array}$} & \multirow[t]{2}{*}{$\mathbf{R}^{2}$} & \multirow{2}{*}{$\begin{array}{c}\mathbf{R}^{2} \\
\text { korr. }\end{array}$} & \multirow{2}{*}{$\begin{array}{c}\text { SFe d. } \\
\text { Schätzers }\end{array}$} & \multirow{2}{*}{$\begin{array}{l}\text { Ände- } \\
\text { rung } R^{2}\end{array}$} & \multirow{2}{*}{$\begin{array}{l}\text { Ände- } \\
\text { rung F }\end{array}$} & \multirow[t]{2}{*}{ df 1} & \multirow[t]{2}{*}{ df 2} & \multirow{2}{*}{$\begin{array}{c}\text { Änderung } \\
\text { F-Sign. }\end{array}$} & \multicolumn{2}{|c|}{ Ausgeschlossen } \\
\hline & & & & & & & & & $t$-Wert & sign. \\
\hline Gesamt & .637 & .605 & 9.3 & .637 & 20.2 & 4 & 46 & .000 & & \\
\hline step: & & & & & & & & & & \\
\hline MO46 & .485 & .475 & 10.7 & .485 & 46.2 & 1 & 49 & .000 & & \\
\hline TRI & .587 & .570 & 9.7 & .102 & 11.9 & 1 & 48 & .001 & & \\
\hline KNII & .621 & .597 & 9.4 & .034 & 4.2 & 1 & 47 & .047 & & \\
\hline WFL & & & & & & & & & 1.43 & .160 \\
\hline
\end{tabular}

LZG: Abruf aus dem Langzeitgedächtnis; WFL: Wortflüssigkeitstest

\begin{tabular}{|c|c|c|c|c|c|c|c|c|c|c|}
\hline \multirow{2}{*}{$\begin{array}{l}\text { BW + } \\
\text { LZG }\end{array}$} & \multirow[t]{2}{*}{$\mathbf{R}^{2}$} & \multirow{2}{*}{$\begin{array}{c}\mathbf{R}^{2} \\
\text { korr. }\end{array}$} & \multirow{2}{*}{$\begin{array}{c}\text { SFe d. } \\
\text { Schätzers }\end{array}$} & \multirow{2}{*}{$\begin{array}{l}\text { Ände- } \\
\text { rung } R^{2}\end{array}$} & \multirow{2}{*}{$\begin{array}{l}\text { Ände- } \\
\text { rung F }\end{array}$} & \multirow[t]{2}{*}{$\overline{d f} 1$} & \multirow[t]{2}{*}{$\overline{\mathrm{df} 2}$} & \multirow{2}{*}{$\begin{array}{c}\text { Änderung } \\
\text { F-Sign. }\end{array}$} & \multicolumn{2}{|c|}{ Ausgeschlossen } \\
\hline & & & & & & & & & t-Wert & sign. \\
\hline Gesamt & .702 & .676 & 8.4 & .702 & 27.1 & 4 & 46 & .000 & & \\
\hline step: & & & & & & & & & & \\
\hline WE & .558 & .549 & 9.9 & .558 & 61.9 & 1 & 49 & .000 & & \\
\hline WT & .663 & .649 & 8.8 & .105 & 14.9 & 1 & 48 & .000 & & \\
\hline WK & .696 & .676 & 8.4 & .033 & 5.1 & 1 & 47 & .028 & & \\
\hline WFL & & & & & & & & & $\begin{array}{l}-.1 .0 \\
\end{array}$ & .335 \\
\hline
\end{tabular}

WE: Laute Ersetzen; Laute Vertauschen; WK: Laute Klassifizieren

\begin{tabular}{|c|c|c|c|c|c|c|c|c|c|c|}
\hline \multirow{2}{*}{$\begin{array}{l}\mathrm{AG}+\mathrm{BW} \\
+\mathrm{LZG}\end{array}$} & \multirow[t]{2}{*}{$\mathbf{R}^{2}$} & \multirow{2}{*}{$\begin{array}{c}\mathbf{R}^{2} \\
\text { korr. }\end{array}$} & \multirow{2}{*}{$\begin{array}{c}\text { SFe d. } \\
\text { Schätzers }\end{array}$} & \multirow{2}{*}{$\begin{array}{l}\text { Ände- } \\
\text { rung } R^{2}\end{array}$} & \multirow{2}{*}{$\begin{array}{l}\text { Ãnde- } \\
\text { rung F }\end{array}$} & \multirow[t]{2}{*}{ df 1} & \multirow[t]{2}{*}{ df 2} & \multirow{2}{*}{$\begin{array}{c}\text { Änderung } \\
\text { F-Sign. }\end{array}$} & \multicolumn{2}{|c|}{ Ausgeschlossen } \\
\hline & & & & & & & & & $t$-Wert & sign. \\
\hline Gesamt & .781 & .745 & 7.5 & .781 & 21.8 & 7 & 43 & .000 & & \\
\hline $\begin{array}{ll}\text { Gesamt } \\
\text { WR u. WK }\end{array}$ & .772 & .741 & 7.5 & .772 & 24.8 & 6 & 44 & .000 & & \\
\hline step: & & & & & & & & & & \\
\hline WE & .558 & .549 & 9.9 & .558 & 61.9 & 1 & 49 & .000 & & \\
\hline WT & .663 & .649 & 8.8 & .105 & 14.9 & 1 & 48 & .000 & & \\
\hline MO46 & .727 & .710 & 8.0 & .064 & 11.1 & 1 & 47 & .002 & & \\
\hline TRI & .751 & .729 & 7.7 & .023 & 4.3 & 1 & 46 & .043 & & \\
\hline KNII & .768 & .742 & 7.5 & .017 & 3.3 & 1 & 45 & .075 & & \\
\hline WFL & & & & & & & & & -.89 & .379 \\
\hline WK & & & & & & & & & .99 & .328 \\
\hline
\end{tabular}

WR: Restwort-Aufgabe

Tab. 24: Kombination der kognitionspsychologischen Konstrukte anhand ihrer aggregierten Variablen bei der Erklärung der Rechtschreibleistung.

Die Zeile „Verarbeitungsebene Gesamt“ gibt das Erklärungspotential wieder, das sich durch das Einbeziehen aller Variablen ergibt. Die darunterstehenden Zeilen (,step“) beschreiben die Regression beim schrittweises Hinzuziehen der einzelnen aggregierten Variablen. In den beiden rechten Spalten finden sich die für den Ausschluss verantwortlichen Werte ausgeschlossener Variablen.

$\mathrm{R}^{2}$ (korr.): (korrigierte) Varianzaufklärung; $\mathrm{S}_{\mathrm{Fe}}$ d. Schätzers: Standardfehler des Schätzers; Änderung F: Änderung des F-Werts; Änderung F-Sign.: Änderung der Signifikanz des F-Werts; o.: ohne 


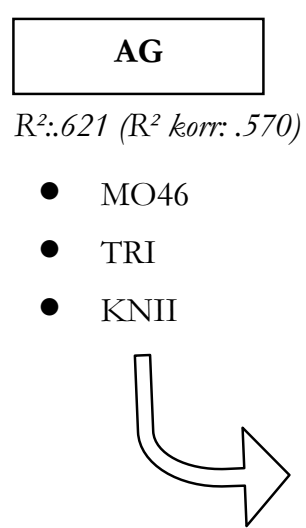

$R^{2} .768$ (R² korr: .742)

- WE

- WT

- MO46

- TRI

- KNII

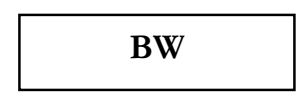

$R^{2} .696\left(R^{2}\right.$ korr: .676)

- WE

- WT

- WK

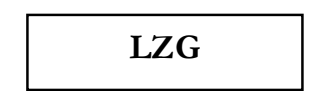

$\mathrm{R}^{2}: 283$ ( $\mathrm{R}^{2}$ korr: .286)

- WFL

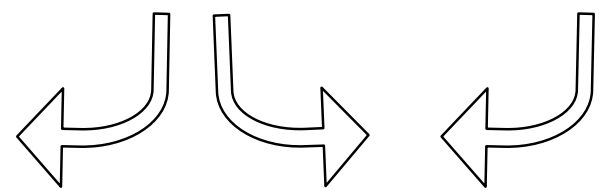

$R^{2} .696\left(R^{2}\right.$ korr: .676)

- WE

- WT

- WK

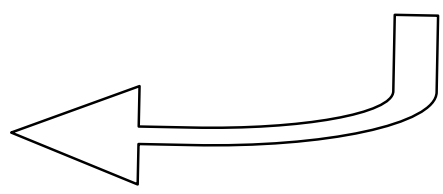

$R^{2} .621$ ( $R^{2}$ korr: .597)

- MO46

- TRI

- KNII

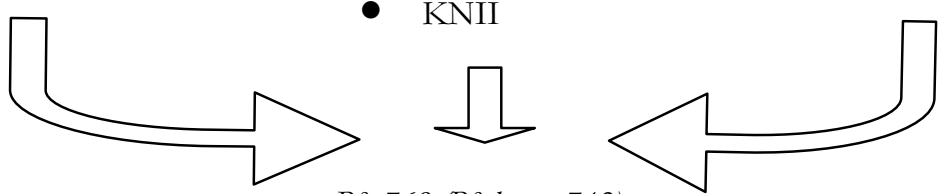

$R^{2}: .768$ (R² korr: .742)

- WE

- WT

- MO46

- TRI

- KNII

Abb. 14: Kombinationen der kognitionspsychologischen Konstrukte anhand ihrer jeweils aggregierten Variablen bei der Erklärung der Rechtschreibleistung.

Konvergierende Pfeile versinnbildlichen die Kombination von Ebenen.

AG: Arbeitsgedächtnis; BW: phonologische Bewusstheit; LZG: Abruf aus dem Langzeitgedächtnis; MO46: 4bis 6silbige Items des Mottier; TRI: Trigramme; KNII: Interaktionswert; WE: Laute Ersetzen, WT: Laute Vertauschen, WK: Laute Klassifizieren; WFL: Wortflüssigkeitstest $\mathrm{R}^{2}$ (korr.): (korrigierte) Varianzaufklärung. 
Die Kombination der aggregierten Variablen des Arbeitsgedächtnisses und der phonologischen Bewusstheit erfolgte beinahe unter Erhalt aller Ausgangsvariablen; lediglich das Laute Klassifizieren entfiel. Das bedeutet, dass für jedes der beiden ohnehin schon erklärungskräftigen Konstrukte die Hinzunahme des jeweils anderen dennoch eine signifikante Vorhersageverbesserung bedingte.

Gemeinsam erreichten sie einen Regressionswert von $\mathrm{R}^{2}=.768\left(\mathrm{R}^{2}\right.$ korr $\left.=.742\right)$, der um nur etwa .07 über dem lag, was von der phonologischen Bewusstheit alleine bzw. ca. .15 über dem, was vom Arbeitsgedächtnis alleine erreicht worden war. Die $.07 \mathrm{bzw}$. die .15 stellten den obigen Überlegungen zufolge offenbar diejenigen Varianzbereiche dar, die nur vom Arbeitsgedächtnis bzw. nur von der phonologischen Bewusstheit eingenommen wurden. In den restlichen Anteilen überschnitten sich die Konstrukte.

Bei der Kombination von Arbeitsgedächtnis und Abruf aus dem Langzeitgedächtnis erwies sich der Langzeitgedächtnistest als überflüssig; die Stepwise-Prozedur wählte nur die Arbeitsgedächtnis-Variablen aus. Unter erzwungener Hinzunahme des Wortflüssigkeitstest lag das Erklärungspotenzial der Kombination um den unbedeutenden Regressionswert von .016 höher als ohne den Test.

Dasselbe Bild entstand beim Kombinieren von phonologischer Bewusstheit und Langzeitgedächtnis-Aktivation. Der Erklärungsverbesserung durch die erzwungene Hinzunahme des Wortflüssigkeitstest betrug hier einen Regressionswert von .006.

Der Varianzbereich des Abrufs aus dem Langzeitgedächtnis schien demzufolge hauptsächlich in einen Bereich zu fallen, der bereits sowohl vom Arbeitsgedächtnis als auch von der phonologischen Bewusstheit abgedeckt wurde.

Die minimalen Anteile müssten außerhalb des Arbeitsgedächtnisses zu lokalisieren sein, wobei die.016 große Regression nur außerhalb des Arbeitsgedächtnisses und .006 davon auch außerhalb der phonologischen Bewusstheit liegen müssten. Darauf deutete auch hin, dass die gemeinsame Regression der varianztragenden Tests von Arbeitsgedächtnis und phonologischer Bewusstheit ohne den Wortflüssigkeitstest um .004 niedriger lag $\left(\mathrm{R}^{2}=.768 ; \mathrm{R}^{2}{ }_{\text {korr }}=.742\right)$ als mit diesem Test $\left(\mathrm{R}^{2}=.772 ; \mathrm{R}^{2}\right.$ korr $\left.=.741\right)$.

Aufgrund ihrer Geringfügigkeit waren diese Beträge inhaltlich nicht von Belang, werden aber dennoch erwähnt, damit eventuelle Unstimmigkeiten bei Vergleichen leichter eingeordnet werden können. Ebenfalls um Unstimmigkeiten möglichst gering zu halten, wurden sie auch in die Rechnungen einbezogen. 
Die Verknüpfung aller drei kognitionspsychologischen Konstrukte bestätigte die Ergebnisse der Zweier-Kombinationen: Die Einbeziehung des Wortflüssigkeitstests und des Laute Klassifizierens brachte den geringen Erklärungszugewinn einer Regression von .013 und wurde damit nicht als bedeutsam erachtet.

Das sich ergebende Bild über das Ineinandergreifen der Regressionsbereiche von phonologischer Bewusstheit, Arbeitsgedächtnis und Abruf aus dem Langzeitgedächtnis bei der Erklärung der Rechtschreibleistung sollte vor der Aufstellung eines Schemas verifiziert werden. Wenn Bereiche sich überschneiden und die Schätzungen der jeweiligen Varianzquantitäten sowie der Überschneidungsbereiche in etwa korrekt sind, so sollte einem Residuum einer Regression des Teils X auf einen Teil Y nur etwa so viel an Varianzerklärung (an der Rechtschreibleistung) bleiben, wie sich aus der Subtraktion von $\mathrm{X}$ und $\mathrm{Y}$ ergibt.

Dieses Prinzip wurde zur Verifikation der obigen Schätzungen angewandt. Tabelle 25 gibt die Erwartungen und Resultate wieder.

\begin{tabular}{||l|c|c|c|c|c|c|c|c|c|}
\hline VERIFIKATION & $\mathbf{E}$ & $\mathbf{R}^{\mathbf{2}}$ & $\begin{array}{c}\mathbf{R}^{\mathbf{2}} \\
\text { korr. }\end{array}$ & $\begin{array}{c}\text { SFe d. } \\
\text { Schätzers }\end{array}$ & $\begin{array}{c}\text { Ände- } \\
\text { rung } \mathbf{R}^{2}\end{array}$ & $\begin{array}{c}\text { Ände- } \\
\text { rung F }\end{array}$ & df 1 & df 2 & $\begin{array}{c}\text { Ände- } \\
\text { rung F- } \\
\text { Sign. }\end{array}$ \\
\hline LZG - BW & $\sim .006$ & $\mathbf{. 0 0 6}$ & -.014 & 14.9 & .006 & .3 & 1 & 49 & .584 \\
\hline LZG - AG & $\sim .016$ &. $\mathbf{0 1 6}$ & -.004 & 14.8 & .016 & .8 & 1 & 49 & .374 \\
\hline AG - BW & $\sim .072$ & $\mathbf{. 0 7 7}$ & .018 & 14.6 & .077 & 1.3 & 3 & 47 & .284 \\
\hline AG - LZG & $\sim .354$ &. $\mathbf{3 5 4}$ & .313 & 12.2 & .354 & 8.6 & 3 & 47 & .000 \\
\hline BW - LZG & $\sim .419$ &. $\mathbf{4 1 9}$ & .382 & 11.6 & .419 & 11.3 & 3 & 47 & .000 \\
\hline BW - AG & $\sim .147$ &. $\mathbf{1 5 2}$ & .098 & 14.0 & .152 & 2.8 & 3 & 47 & .050 \\
\hline
\end{tabular}

Tab. 25: Verifikation der Überlegungen zur Interaktion der kognitionspsychologischen Konstrukte.

$\mathrm{R}^{2}$ (korr.): (korrigierte) Varianzaufklärung; $\mathrm{S}_{\mathrm{Fe}}$ d. Schätzers: Standardfehler des Schätzers; Änderung F: Änderung des F-Werts; Änderung F-Sign.: Änderung der Signifikanz des F-Werts

AG: Arbeitsgedächtnis; BW: phonologische Bewusstheit; LZG: Abruf aus dem Langzeitgedächtnis. E: Erwartungen für $\mathrm{R}^{2}$

Berechnung der Erwartungswerte:

$\mathrm{LZG}-\mathrm{BW}:(\mathrm{BW}+\mathrm{LZG})_{\mathrm{ges}}-(\mathrm{BW}+\mathrm{LZG})_{\mathrm{step}}=.702-.696 \quad=.006$

$\mathrm{LZG}-\mathrm{AG}:(\mathrm{AG}+\mathrm{LZG})_{\mathrm{ges}}-(\mathrm{AG}+\mathrm{LZG})_{\mathrm{step}}=.637-.621 \quad=.016$

$\mathrm{AG}-\mathrm{BW}:$ ALLE - BW step $=.768-.696 \quad=.072$

$\mathrm{AG}-\mathrm{LZG}: \mathrm{AG}$ step $-\mathrm{LZG}$ step $+(\mathrm{LZG}-\mathrm{AG})=.621-.283+.016=.354$

$\mathrm{BW}-\mathrm{LZG}: \mathrm{BW}_{\mathrm{step}}-\mathrm{LZG}_{\mathrm{step}}+(\mathrm{LZG}-\mathrm{BW})=.696-.283+.006=.419$

$\mathrm{BW}-\mathrm{AG}:(\mathrm{AG}+\mathrm{BW})_{\text {step }}-\mathrm{AG}$ step $\quad=.768-.621=.147$

Es wurde deutlich, dass die im Verifikationsverfahren erhaltenen Werte ausgesprochen nah an den Werten lagen, die auf der Basis der vorangegangenen Ergebnisse erwartet wurden. Es konnte demzufolge von der Richtigkeit der Überlegungen ausgegangen werden, welche in folgendem Schema visualisiert wurden (Abb. 15): 


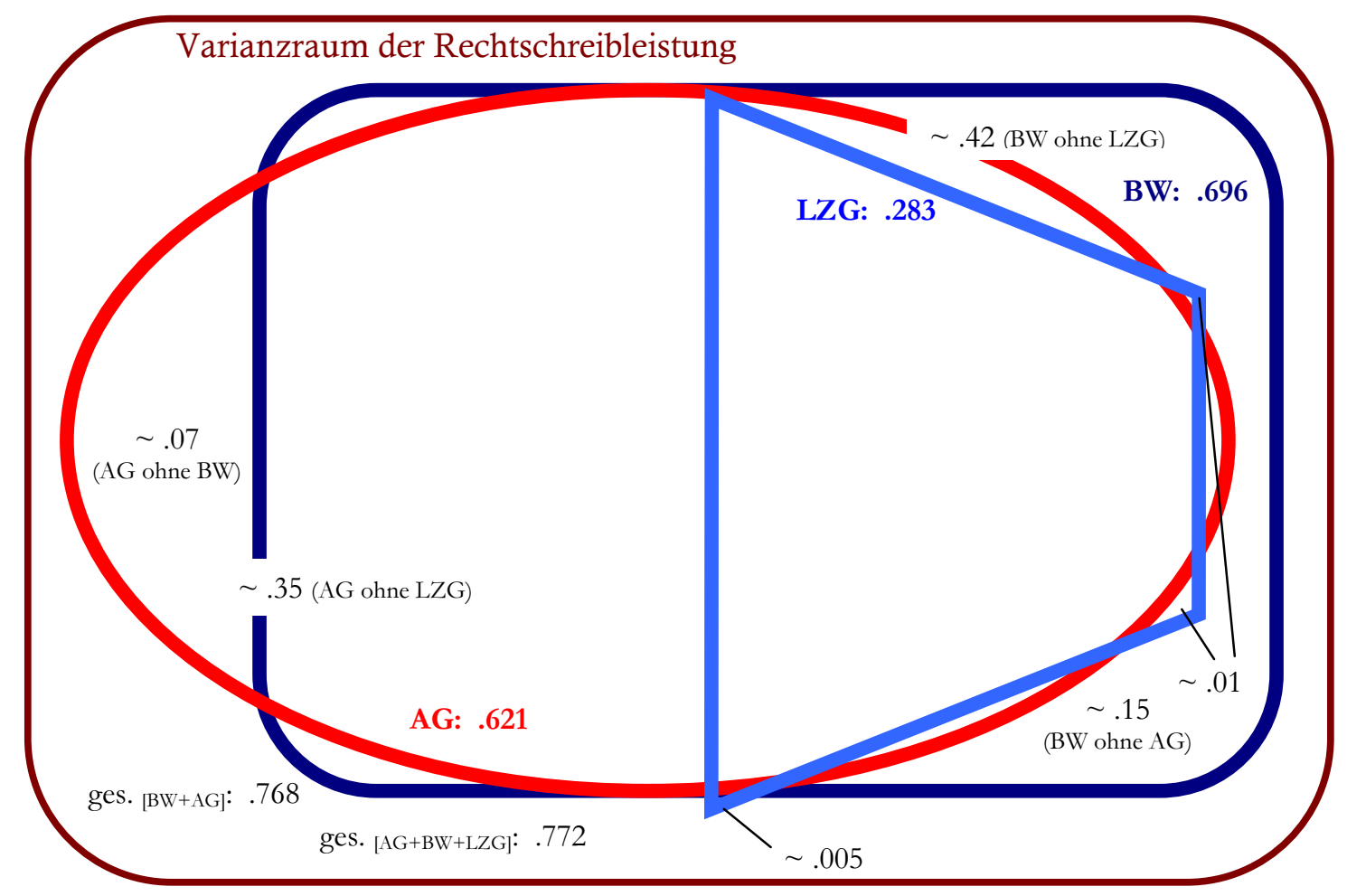

Abb. 15: Mögliche Struktur der Erklärungspotentiale der kognitionspsychologischen Konstrukte im Varianzraum der Rechtschreibung.

Fett- und buntgedruckt sind die Beträge der unkorrigierten Varianzerklärung der Konstrukte.

Im Normaldruck sind die Schätzwerte für Teilbereiche angegeben. Diese Werte sind gerundet.

Anmerkung: Varianzanteile der Konstrukte bzw. Variablen, die nicht mit der Rechtschreibleistung in

Verbindung stehen, sind hier nicht abgebildet.

AG: phonologisches Arbeitsgedächtnis; BW: phonologische Bewusstheit; LZG: Zugriff auf das Langzeitgedächtnis.

\subsubsection{IMPLEMENTIERUNG DER EEG-VARIABLEN IN DAS VARIANZ- SCHEMA DER KOGNITIONSPSYCHOLOGISCHEN KONSTRUKTE}

\subsection{REGRESSION DER EINZELNEN EEG-VARIABLEN}

Um die Frage zu beantworten, wie sich die EEG-Variablen in das aufgestellte Schema bezüglich der Interaktion von Rechtschreibvarianz-Anteilen der kognitionspsychologischen Konstrukte einfügen lassen, war in einem ersten Schritt interessant, wie hoch das Erklärungspotenzial jeder einzelnen Variable einzuschätzen war. Tabelle 26 gibt hierauf Antwort. 


\begin{tabular}{|l|c|c|c|c|c|c|c|c|}
\hline & $\mathbf{R}^{\mathbf{2}}$ & $\begin{array}{c}\mathbf{R}^{\mathbf{2}} \\
\text { korr. }\end{array}$ & $\begin{array}{c}\text { SFe d. } \\
\text { Schätzers }\end{array}$ & $\begin{array}{c}\text { Ände- } \\
\text { rung R }\end{array}$ & $\begin{array}{c}\text { Ände- } \\
\text { rung F }\end{array}$ & $\mathbf{d f ~ 1 ~}$ & df 2 & $\begin{array}{c}\text { Änderung } \\
\text { F-Sign. }\end{array}$ \\
\hline PHO & $\mathbf{. 0 6 1}$ & .041 & 14.5 & .061 & 3.1 & 1 & 47 & .087 \\
\hline NP & .117 & .098 & 14.0 & .117 & 6.2 & 1 & 47 & .016 \\
\hline SIN & .116 & .096 & 14.0 & .116 & 6.0 & 1 & 46 & .018 \\
\hline
\end{tabular}

Tab. 26: Regression der EEG-Variablen auf die Rechtschreibleistung

$\mathrm{R}^{2}$ (korr.): (korrigierte) Varianzaufklärung; $\mathrm{S}_{\mathrm{Fe}}$ d. Schätzers: Standardfehler des Schätzers; Änderung F: Änderung des F-Werts; Änderung F-Sign.: Änderung der Signifikanz des F-Werts.

PHO: Peaklatenz der MMN in PH-G, Segment B; NP: Fläche der MMN in NP-D, Segment B; SIN: Fläche der MMN in SIN-D.

Die nichtphonologische und die Sinus-Variable erreichten so gut wie gleich hohe Erklärungspotenziale, nämlich $.117\left(\mathrm{R}^{2}{ }_{\text {korr }}=.098 ; \mathrm{NP}\right)$ und $.116\left(\mathrm{R}^{2}{ }_{\text {korr }}=.096 ; \mathrm{SIN}\right)$. Halb so viel Varianz erklärte die phonologische Variable $.061\left(\mathrm{R}^{2}{ }_{\text {korr }}=.041\right)$.

\subsection{GEGENSEITIGE ÜBERSCHNEIDUNG DER EEG-VARIANZEN}

Die Addition dieser Beträge führt zu einem Wert von .294. Auf .283 war das Gesamtpotenzial der EEG-Variablen in Abschnitt 5.3.2.1. geschätzt worden. Die Differenz ist unerheblich, so dass man kaum von der Existenz bedeutender Überschneidungsbereiche ausgehen würde.

Nichtsdestotrotz waren diese vorhanden, wie Tabelle 27 zeigt. Hier wurde -ähnlich wie im vorangegangenen Abschnitt- die nach einer Regression einer auf eine andere Variable die dem Residuum bleibende Varianzaufklärung an der Rechtschreibleistung ermittelt und mit dem Ausgangswert verglichen. Negative Werte in der Spalte „d $\mathrm{R}^{2 “}$ indizieren Varianzminderungen durch die Variablen-Extraktion.

\begin{tabular}{|l|c|c|c|c|c|c|c|c|c|}
\hline & $\mathbf{R}^{\mathbf{2}}$ & $\mathbf{d ~ R}^{\mathbf{2}}$ & $\begin{array}{c}\mathbf{R}^{\mathbf{2}} \\
\text { korr. }\end{array}$ & $\begin{array}{c}\text { SFe d. } \\
\text { Schätzers }\end{array}$ & $\begin{array}{c}\text { Ände- } \\
\text { rung } \mathbf{R}^{\mathbf{2}}\end{array}$ & $\begin{array}{c}\text { Ände- } \\
\text { rung F }\end{array}$ & $\mathbf{\text { df 1 }}$ & $\mathbf{\text { df 2 }}$ & $\begin{array}{c}\text { Ände- } \\
\text { rung F- } \\
\text { Sign. }\end{array}$ \\
\hline PHO & $\mathbf{. 0 6 1}$ & & .041 & 14.4 & .061 & 3.1 & 1 & 47 & .087 \\
\hline PHO - NP & $\mathbf{. 0 6 9}$ & $\mathbf{+ . 0 0 8}$ & .049 & 14.4 & .069 & 3.5 & 1 & 47 & .069 \\
\hline PHO - SIN & $\mathbf{. 1 0 2}$ & $\mathbf{+ . 0 4 1}$ & .083 & 14.1 & .102 & 5.2 & 1 & 46 & .027 \\
\hline NP & $\mathbf{. 1 1 7}$ & & .098 & 14.0 & .117 & 6.2 & 1 & 47 & .016 \\
\hline NP - PHO & $\mathbf{. 1 2 5}$ & $\mathbf{+ . 0 0 8}$ & .106 & 14.0 & .125 & 6.7 & 1 & 47 & .013 \\
\hline NP - SIN & $\mathbf{. 0 6 7}$ & $\mathbf{- . 0 5 0}$ & .047 & 14.4 & .067 & 3.3 & 1 & 46 & .076 \\
\hline SIN & $\mathbf{. 1 1 6}$ & & .096 & 14.0 & .116 & 6.0 & 1 & 46 & .018 \\
\hline SIN - PHO & $\mathbf{. 1 4 4}$ & $\mathbf{+ . 0 2 8}$ & .126 & 13.8 & .144 & 7.8 & 1 & 46 & .008 \\
\hline SIN - NP & $\mathbf{. 0 7 8}$ & $\mathbf{- . 0 3 8}$ & .058 & 14.3 & .078 & 3.9 & 1 & 46 & .054 \\
\hline
\end{tabular}

Tab. 27: Gegenseitiger Einfluss der EEG-Variablen im Varianzraum der Rechtschreibleistung.

$\mathrm{d} \mathrm{R}^{2}$ : Regressionsdifferenz zum unbereinigten Stimulus; R ${ }^{2}$ (korr.): (korrigierte) Varianzaufklärung; $\mathrm{S}_{\mathrm{Fe}} \mathrm{d}$. Schätzers: Standardfehler des Schätzers; Änderung F: Änderung des F-Werts; Änderung F-Sign.: Änderung der Signifikanz des F-Werts

PHO: Peaklatenz der MMN in PH-G, Segment B; NP: Fläche der MMN in NP-D, Segment B; SIN: Fläche der MMN in SIN-D. 
Erstaunlich ist, dass es, wenn die Variable PHO beteiligt war -und dies besonders in der Interaktion mit der Variable SIN-, durch die Extraktionen auch zu Varianzmaximierungen kam. In der Hoffnung, varianzstärkere oder bezüglich der Gruppenunterschiede aussagekräftigere EEG-Variablen zu erhalten, wurde diese Fährte sogleich weiterverfolgt. Die Residuen zeigten jedoch keinen Vorteil gegenüber den Rohvariablen.

Auch wenn dieser Befund offenbar keine Basis für Alternativvariablen stellte, so ließ er sich dennoch nutzen um zu erklären, weshalb das Gesamterklärungspotenzial der EEG-Variablen trotz vorhandener Überschneidungen gegenüber der Summe der Einzelvarianzen nicht (kaum) verringert war.

Die Überschneidungen bestanden zwischen den MMNs der beiden nichtsprachlichen Deviants. Die Extraktion des SIN kostete den NP .050 R²-Anteile; die Extraktion des NP kostete den SIN hingegen nicht ebenso viel, wie es für einen gemeinsamen Bereich anzunehmen gewesen wäre, sondern nur $.038 \mathrm{R}^{2}$-Anteile. In einer Größenordnung zwischen diesen beiden Werten musste eine Überlappung der MMN-Varianzen demzufolge liegen.

\subsection{ADDITIVE VARIANZBEREICHE BEZÜGLICH DER KOGNITIONSPSYCHOLO- GISCHEN KONSTRUKTE}

Bei der Einordnung der MMN-Varianzen in das Schema der kognitionspsychologischen Varianzen an der Rechtschreibleistung taucht weiterhin die Frage auf, ob die EEG-Variablen einen zusätzlichen Beitrag zur Erklärung leisten. Dies wurde mithilfe der Einbeziehung der einzelnen EEG-Variablen in die Regression der tragenden Variablen von Arbeitsgedächtnis und phonologischer Bewusstheit ermittelt. Die Ergebnisse gibt Tabelle 28 wieder.

\begin{tabular}{|l|c|c|c|c|c|c|c|c|}
\hline $\begin{array}{l}\text { [AG + BW + LZG] + } \\
\text { PHO / NP / SIN }\end{array}$ & $\mathbf{R}^{\mathbf{2}}$ & $\begin{array}{c}\mathbf{R}^{2} \\
\text { korr. }\end{array}$ & $\begin{array}{c}\text { SFe d. } \\
\text { Schätzers }\end{array}$ & $\begin{array}{c}\text { Ände- } \\
\text { rung } \mathbf{R}^{\mathbf{2}}\end{array}$ & $\begin{array}{c}\text { Ände- } \\
\text { rung F }\end{array}$ & $\mathbf{d f 1}$ & $\mathbf{d f} 2$ & $\begin{array}{c}\text { Änderung } \\
\text { F-Sign. }\end{array}$ \\
\hline Gesamt & $\mathbf{. 7 9 3}$ & .744 & 7.5 & .793 & 16.2 & 9 & 38 & .000 \\
\hline & & & & & & & & \\
\hline [AG + BW + LZG] & $\mathbf{. 7 7 2}$ & .741 & 7.5 & .772 & 24.8 & 6 & 44 & .000 \\
\hline + PHO & $\mathbf{. 7 8 0}$ & .742 & 7.5 & .780 & 20.7 & 7 & 41 & .000 \\
\hline + NP & .772 & .734 & 7.6 & .772 & 19.9 & 7 & 41 & .000 \\
\hline + SIN & $\mathbf{7 8 1}$ & .743 & 7.5 & .781 & 20.4 & 7 & 40 & .000 \\
\hline + SIN + PHO & $\mathbf{. 7 9 3}$ & .751 & 7.4 & .793 & 18.7 & 8 & 39 & .000 \\
\hline
\end{tabular}

Tab. 28: Regression der kognitionspsychologischen Konstrukte (anhand der aggregierten Variablen) ohne / mit EEG-Variablen auf die Rechtschreibleistung.

AG: phonologisches Arbeitsgedächtnis; BW: phonologische Bewusstheit; LZG: Zugriff auf das Langzeitgedächtnis PHO: Peaklatenz der MMN der Bedingung PH-G; Segment B; NP: Flächenwert der MMN der Bedingung NP-D, Segment B; SIN: Flächenwert der MMN der Bedingung SIN-D.

$\mathrm{R}^{2}$ (korr.): (korrigierte) Varianzaufklärung; $\mathrm{S}_{\mathrm{Fe}} \mathrm{d}$. Schätzers: Standardfehler des Schätzers; Änderung F: Änderung des F-Werts; Änderung F-Sign.: Änderung der Signifikanz des F-Werts 
In der Regression der kognitionspsychologischen Variablen mit allen drei EEG-Variablen konnte ein Wert von insgesamt .793 erzielt werden. Das sind $2.1 \%$ mehr als ohne die EEGVariablen und folglich als deren zusätzlicher -wenn auch sehr geringfügiger- Beitrag anzusehen. Die MMN der Variable NP bewirkte keinerlei Steigerung des Erklärungspotenzials; die MMN von PHO und SIN brachte Steigerungen von .008 bzw. .009. Die ca. .012 des NP mussten daher vollständig innerhalb des bereits durch die kognitions-psychologischen Variablen abgedeckten Bereiches liegen. PHO und SIN waren zwar zu großen Teilen ebenfalls innerhalb dieses Bereichs zu lokalisieren (da ihre Gesamterklärungspotenziale weit größer eingeschätzt wurden, als sie die kognitionspsychologische Gesamtvarianz zu steigern vermochten), verfügten aber auch über geringe Areale außerhalb. Dass die Varianzsteigerung auf den Gesamtwert von .793 wirklich auf die MMN-Variablen PHO und SIN und nicht auf den NP zurückging, bewies die gemeinsame Regressionseinbeziehung von PHO und SIN. Die erklärte Varianz betrug in diesem Fall wieder .793.

\subsection{ZUSAMMENFÜGEN DER EEG-VARIANZEN ZUM VARIANZSCHEMA DER KOGNITIONSPSYCHOLOGISCHEN KONSTRUKTE}

Nun gilt es, die soweit charakterisierten Varianzbereiche der EEG-Stimuli mit den Varianzbereichen der kognitionspsychologischen Variablen zu verbinden. Die methodische Annäherung an diese Aufgabe bestand wieder im Kombinieren zweier Verarbeitungsebenen -das heißt hier eines kognitionspsychologischen Konstrukts mit den drei EEG-Variablen- mithilfe der Stepwise-Regression. Die Rechenschritte sind in Tabelle 29 dokumentiert und der Abbildung 16 veranschaulicht: 


\begin{tabular}{|c|c|c|c|c|c|c|c|c|c|c|}
\hline \multirow[t]{2}{*}{$\mathrm{AG}+\mathrm{EEG}$} & \multirow[t]{2}{*}{$\mathbf{R}^{2}$} & \multirow{2}{*}{$\begin{array}{c}\mathrm{R}^{2} \\
\text { korr. }\end{array}$} & \multirow{2}{*}{$\begin{array}{c}\text { SFe d. } \\
\text { Schät- } \\
\text { zers }\end{array}$} & \multirow{2}{*}{$\begin{array}{l}\text { Ände- } \\
\text { rung } R^{2}\end{array}$} & \multirow{2}{*}{$\begin{array}{l}\text { Ände- } \\
\text { rung F }\end{array}$} & \multirow[t]{2}{*}{ df 1} & \multirow[t]{2}{*}{ df 2} & \multirow{2}{*}{$\begin{array}{l}\text { Ände- } \\
\text { rung F- } \\
\text { Sign. }\end{array}$} & \multicolumn{2}{|c|}{ Ausgeschlossen } \\
\hline & & & & & & & & & t-Wert & sign. \\
\hline Gesamt & .658 & .608 & 9.2 & .658 & 13.1 & 6 & 41 & .000 & & \\
\hline step: & & & & & & & & & & \\
\hline MO46 & .485 & .474 & 10.7 & .485 & 43.3 & 1 & 46 & .000 & & \\
\hline TRI & .587 & .569 & 9.7 & .102 & 11.1 & 1 & 45 & .002 & & \\
\hline KNII & .621 & .595 & 9.4 & .034 & 3.9 & 1 & 44 & .054 & & \\
\hline PHO & & & & & & & & & 1.04 & .304 \\
\hline NP & & & & & & & & & .44 & .663 \\
\hline SIN & & & & & & & & & 1.53 & .133 \\
\hline
\end{tabular}

AG: Arbeitsgedächtnis; MO46: 4- bis 6silbige Items des Mottier; TRI: Trigramme; KNII: Kunstwort-Nachsprechen: Interaktionswert; PHO: Peaklatenz aus PH-G, Segment B; NP: Fläche aus NP-D; Segment B; SIN: Fläche aus SIN-D.

\begin{tabular}{|l|c|c|c|c|c|c|c|c|c|c|}
\hline BW + EEG & $\mathbf{R}^{\mathbf{2}}$ & $\begin{array}{c}\mathbf{R}^{\mathbf{2}} \\
\text { korr. }\end{array}$ & $\begin{array}{c}\text { SFe d. } \\
\text { Schät- } \\
\text { zers }\end{array}$ & $\begin{array}{c}\text { Ände- } \\
\text { rung R }\end{array}$ & $\begin{array}{c}\text { Ände- } \\
\text { rung F }\end{array}$ & df 1 & df 2 & $\begin{array}{c}\text { Ände- } \\
\text { rung F- } \\
\text { Sign. }\end{array}$ & Ausgeschlossen \\
\hline Gesamt & .757 & .722 & 7.8 & .757 & 21.3 & 6 & 41 & .000 & & sign. \\
\hline step: & & & & & & & & & & \\
\hline WE & .558 & .548 & 9.9 & .558 & 58.1 & 1 & 46 & .000 & & \\
\hline WT &. $\mathbf{6 6 3}$ & .648 & 8.8 & .105 & 14.0 & 1 & 45 & .001 & & \\
\hline WK & $\mathbf{. 6 9 6}$ & .675 & 8.4 & .033 & 4.8 & 1 & 44 & .034 & & \\
\hline PHO &. $\mathbf{7 3 1}$ & .706 & 8.0 & .035 & 5.6 & 1 & 43 & .022 & & \\
\hline NP & & & & & & & & & 1.5 & .152 \\
\hline SIN & & & & & & & & & 1.7 & .106 \\
\hline
\end{tabular}

BW: phonologische Bewusstheit; WE: / WT / WK: Würzburger Testbatterie: Laute Ersetzen / Laute Vertauschen / Laute Klassifizieren.

\begin{tabular}{|c|c|c|c|c|c|c|c|c|c|c|}
\hline \multirow[t]{2}{*}{$\mathrm{LZG}+\mathrm{EEG}$} & \multirow[t]{2}{*}{$\mathbf{R}^{2}$} & \multirow{2}{*}{$\begin{array}{c}\mathrm{R}^{2} \\
\text { korr. }\end{array}$} & \multirow{2}{*}{$\begin{array}{c}\text { SFe d. } \\
\text { Schät- } \\
\text { zers }\end{array}$} & \multirow{2}{*}{$\begin{array}{l}\text { Ände- } \\
\text { rung } R^{2}\end{array}$} & \multirow{2}{*}{$\begin{array}{l}\text { Ände- } \\
\text { rung F }\end{array}$} & \multirow[t]{2}{*}{ df 1} & \multirow[t]{2}{*}{ df 2} & \multirow{2}{*}{$\begin{array}{l}\text { Ände- } \\
\text { rung F- } \\
\text { Sign. }\end{array}$} & \multicolumn{2}{|c|}{ Ausgeschlossen } \\
\hline & & & & & & & & & $\mathrm{t}$-Wert & sign. \\
\hline Gesamt & .443 & .391 & 11.5 & .443 & 8.5 & 4 & 43 & .000 & & \\
\hline \multicolumn{11}{|l|}{ step: } \\
\hline WFL & .283 & .268 & 12.6 & .283 & 18.2 & 1 & 46 & .000 & & \\
\hline NP & .354 & .326 & 12.1 & .071 & 5.0 & 1 & 45 & .031 & & \\
\hline PHO & .396 & .355 & 11.9 & .042 & 3.1 & 1 & 44 & .086 & & \\
\hline SIN & .443 & .391 & 11.5 & .046 & 3.6 & 1 & 43 & .065 & & \\
\hline
\end{tabular}

LZG: Abruf aus dem Langzeitgedächtnis; WFL: Wortflüssigkeitstest.

$\mathrm{R}^{2}$ (korr.): (korrigierte) Varianzaufklärung; $\mathrm{S}_{\mathrm{Fe}}$ d. Schätzers: Standardfehler des Schätzers; Änderung F: Änderung des F-Werts; Änderung F-Sign.: Änderung der Signifikanz des F-Werts.

\section{Tab. 29:}

Kombination der kognitionspsychologischen Konstrukte mit der neurophysiologischen Verarbeitungsebene anhand ihrer aggregierten Variablen zur Erklärung der Rechtschreibleistung.

Die Zeile „Verarbeitungsebene Gesamt“ gibt das Erklärungspotential wieder, das sich durch das Einbeziehen aller Variablen ergibt. Die darunterstehenden Zeilen (,step") beschreiben die Regression bei schrittweisem Hinzuziehen der einzelnen Variablen. In den beiden rechten Spalten finden sich die für den Ausschluss verantwortlichen Werte ausgeschlossener Variablen. 


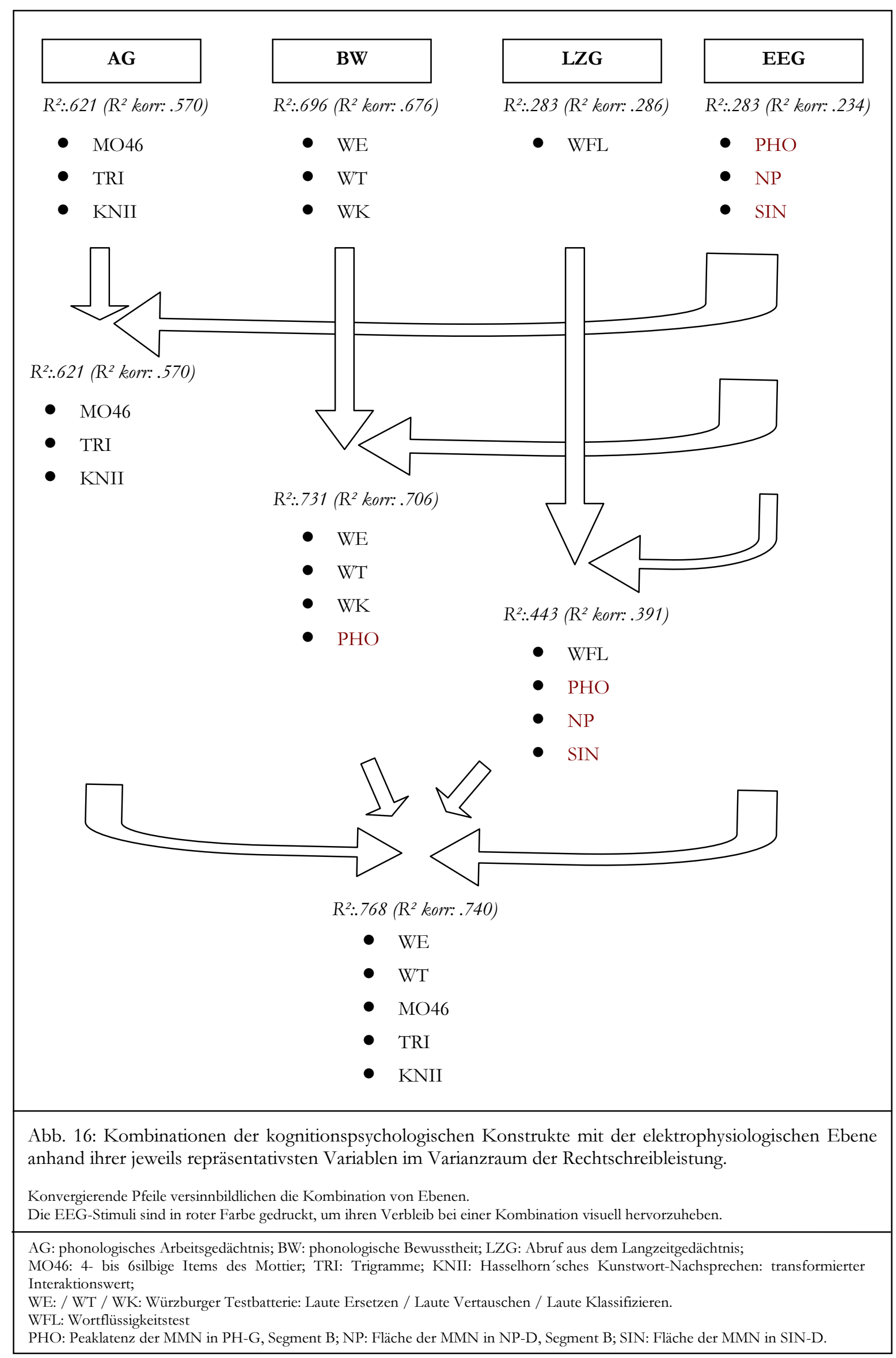


Die auf das Arbeitsgedächtnis zurückgehende Rechtschreibvarianz ließ sich durch die Einbeziehung von EEG-Variablen offenbar gar nicht signifikant vergrößern. Dies bedeutete, dass sich die Varianzbereiche der EEG-Variablen mit denen des Arbeitsgedächtnisses weitgehend deckten.

Die Vorhersageverbesserung der phonologischen Bewusstheit um einen Regressionswert von .035 durch die Variable PHO erkannte das Stepwise-Verfahren durchaus als signifikant an. Die anderen beiden MMN-Variablen bewirkten allerdings keine nennenswerte Verbesserung. Dies legte den Schluss nahe, dass die Variablen NP und SIN zu ihren Hauptteilen innerhalb eines Überschneidungsbereichs der phonologischen Bewusstheit und allenfalls unbedeutende Anteile außerhalb dessen zu lokalisieren waren, und dass für die Variable PHO das Gegenteil gelten musste.

Die auf den Abruf aus dem Langzeitgedächtnis zurückgehende Rechtschreibvarianz war kleiner und ließ sich schon aus diesem Grund eher als die anderen beiden Konstrukte durch Hinzunahme weiterer Variablen vergrößern. Dass alle EEG-Variablen die Vorhersage signifikant verbesserten, bedeutete außerdem, dass, wenn auch EEG-Bereiche innerhalb der Langzeitgedächtnis-Varianz liegen mochten, auch bedeutsame Anteile außerhalb vorhanden sein mussten.

Zusammenfassend lässt sich nun über das Bild, das durch die Ebenen-Kombination entstand, Folgendes festhalten: Die Arbeitsgedächtnisvarianz schien weitgehend alle EEG-Varianz abzudecken; für die phonologische Bewusstheit traf dies nur für den PHO nicht zu. Bezüglich des Abrufs aus dem Langzeitgedächtnis schien keine EEG-Varianz so gelagert zu sein, dass deren Hinzunahme die Ausgangsvarianzerklärung nicht anheben würde.

Dieses Bild ist allerdings noch relativ unpräzise. Der Inhalt des abschließenden Schrittes bestand folglich in der Präzisierung von Teilbereichen, was gleichzeitig der Absicherung der obigen Überlegungen diente.

Als Verfahren wurde zu diesem Zweck wieder die Residualtechnik angewandt: Die Differenz zwischen Regressionsresiduen der Vorhersage von EEG-Variablen auf kognitionspsychologische Konstrukte und der Ausgangsvarianz dieser Konstrukte sollte Auskunft darüber geben, in welchem Maße eine EEG-Varianz innerhalb des Areals eines Konstrukts Raum beanspruchte.

Die Berechnungen sind in Tabelle 30 nachzuvollziehen: 


\begin{tabular}{|c|c|c|c|c|c|c|c|c|c|}
\hline & $\mathbf{R}^{2}$ & $\mathrm{~d} \mathrm{R}^{2}$ & $\begin{array}{c}\mathbf{R}^{2} \\
\text { korr. }\end{array}$ & $\begin{array}{c}\text { SFe d. } \\
\text { Schät- } \\
\text { zers }\end{array}$ & $\begin{array}{c}\text { Ände- } \\
\text { rung } \\
\mathbf{R}^{2}\end{array}$ & $\begin{array}{l}\text { Ände- } \\
\text { rung } \\
\text { F }\end{array}$ & df 1 & df 2 & $\begin{array}{l}\text { Ände- } \\
\text { rung F- } \\
\text { Sign. }\end{array}$ \\
\hline AG & .621 & & & & & & & & \\
\hline AG - PHO & .565 & .056 & .536 & 10.1 & .565 & 19.5 & 3 & 45 & .000 \\
\hline AG-NP & .501 & .120 & .468 & 10.8 & .501 & 15.1 & 3 & 45 & .000 \\
\hline AG - SIN & .511 & .110 & .478 & 10.7 & .511 & 15.4 & 3 & 44 & .000 \\
\hline BW & .696 & & .676 & 8.4 & .696 & 35.8 & 3 & 47 & .000 \\
\hline BW - PHO & .667 & .019 & .645 & 8.8 & .667 & 30.0 & 3 & 45 & .000 \\
\hline BW-NP & .586 & .110 & .558 & 9.8 & .586 & 21.2 & 3 & 45 & .000 \\
\hline BW - SIN & .585 & .111 & .557 & 9.8 & .585 & 20.7 & 3 & 44 & .000 \\
\hline LZG & .283 & & .268 & 12.6 & .283 & 19.4 & 1 & 49 & .000 \\
\hline LZG - PHO & .276 & .007 & .260 & 12.7 & .276 & 17.9 & 1 & 47 & .000 \\
\hline LZG - NP & .254 & .029 & .238 & 12.9 & .254 & 16.0 & 1 & 47 & .000 \\
\hline LZG - SIN & .215 & .068 & .198 & 13.2 & .215 & 12.6 & 1 & 46 & .001 \\
\hline $\begin{array}{l}{[\mathrm{AG}+\mathrm{BW}+\mathrm{LZG}]-\mathrm{SIN}-} \\
\mathrm{NP}\end{array}$ & .582 & .190 & .520 & 10.2 & .582 & 9.5 & 6 & 41 & .000 \\
\hline AG - EEG & .355 & .266 & .311 & 12.3 & 355 & 8.1 & 3 & 44 & .000 \\
\hline$\left[\begin{array}{l}\mathrm{AG}+\mathrm{BW}+\mathrm{LZG}]-\mathrm{EEG} \\
{[\mathrm{AG}+\mathrm{BW}+\mathrm{LZG}]}\end{array}\right.$ & $\begin{aligned} .504 \\
.772\end{aligned}$ & 268 & .432 & 11.1 & .504 & 6.9 & 6 & 41 & .000 \\
\hline$[\mathrm{AG}+\mathrm{BW}+\mathrm{LZG}]-\mathrm{PHO}$ & .715 & .057 & .675 & 8.4 & .715 & 17.6 & 6 & 42 & .000 \\
\hline$[\mathrm{AG}+\mathrm{BW}+\mathrm{LZG}]-\mathrm{NP}$ & .652 & .120 & .602 & 9.3 & .652 & 13.1 & 6 & 42 & .000 \\
\hline$[\mathrm{AG}+\mathrm{BW}+\mathrm{LZG}]-\mathrm{SIN}$ & .675 & .097 & .627 & 9.0 & .675 & 14.2 & 6 & 41 & .000 \\
\hline
\end{tabular}

Tab. 30: Multiple Regression der EEG-bereinigten kognitionspsychologischen Konstrukte auf die Rechtschreibleistung.

d R R : Differenz des Erklärungspotential zum Erklärungspotential der unbereinigten Verarbeitungskomponente; R ${ }^{2}$ (korr.): (korrigierte) Varianzaufklärung; $\mathrm{S}_{\mathrm{Fe}}$ d. Schätzers: Standardfehler des Schätzers; Änderung F: Änderung des F-Werts; Änderung F-Sign.:

Änderung der Signifikanz des F-Werts;

AG: phonologisches Arbeitsgedächtnis; BW: phonologische Bewusstheit; LZG: Abruf aus dem Langzeitgedächtnis;

PHO: Peaklatenz der MMN in PH-G, Segment B; NP: Fläche der MMN in NP-D, Segment B; SIN: Fläche der MMN in SIN-D.

Das Gesamterklärungspotenzial der Variable PHO wurde in Tabelle 26 auf .061 geschätzt. Gemäß Tabelle 28 war sie zur Erklärung einer .008 großen Regression außerhalb des von den kognitionspsychologischen Variablen abgesteckten Bereichs in der Lage. Um .056 sank hier (Tab. 32) die Arbeitsgedächtnisvarianz nach der Extraktion des PHO. Diese Werte passen recht gut zueinander: Es stellte sich so dar, als ob die .056 der Extraktion aus der Arbeitsgedächtnisvarianz und die .008 außerhalb das vollständige Erklärungspotenzial der Variable PHO erfassten, da ihre Addition den Gesamtwert des PHO bereits mehr als erreichte. Die Varianzeinbußen von .019 der phonologischen Bewusstheit und von .007 des Abrufs aus dem Langzeitgedächtnis wären infolgedessen in Bereichen zu suchen, wo diese mit dem Arbeitsgedächtnis überlappten. Da der Abruf aus dem Langzeitgedächtnis nur mit der phonologischen Bewusstheit überlappte, waren die dortigen .007 wiederum als Teil der .019 der phonologischen 
Bewusstheit $\mathrm{zu}$ sehen. .007 fielen demzufolge in einen Überschneidungsbereich aller drei kognitionspsychologischen Ebenen, .019 - .007 = .012 wären in einem Überschneidungsbereich von phonologischer Bewusstheit und Arbeitsgedächtnis zu suchen und $.056-.019=.037$ lägen in einem allein vom Arbeitsgedächtnis abgedeckten Bereich. Weiterhin würde die Varianz der EEG-Variable durch die bereits erwähnten .008 außerhalb der Gesamtvarianz der kognitionspsychologischen Konstrukte zu ergänzen sein.

Vom geschätzten Erklärungsgesamtpotenzial von ca. .12 $\left(\mathrm{R}^{2}=.117\right)$ der Variable NP war bereits das volle Maß (.120) im Arbeitsgedächtnis zu finden. Somit galt auch für diese Variable, dass die in die phonologische Bewusstheit bzw. in den Abruf aus dem Langzeitgedächtnis fallenden Varianzanteile in Überschneidungsbereichen des Arbeitsgedächtnisses mit den anderen beiden Konstrukten liegen mussten. .110 wurden als Regressionsverlust der phonologischen Bewusstheit, .029 als Regressionsverlust des Abrufs aus dem Langzeitgedächtnis errechnet. . $120-.110=.010$ war folglich die Größe desjenigen Anteils, der allein mit dem Arbeitsgedächtnis Überschneidungen aufwies. Auf $.110-.029=.081$ konnte in etwa der Anteil geschätzt werden, der im Überlappungsbereich von phonologischer Bewusstheit und Arbeitsgedächtnis lag. Im dreifach kognitionspsychologischen Überschneidungsbereich verblieben die direkt errechneten 029 .

Gemäß Tabelle 28 wurde für die SIN-Variable eine außerhalb der kognitionspsychologischen Rechtschreibvarianz befindliche Varianz von .009 ermittelt. Gemeinsam mit der Varianzeinbuße des Arbeitsgedächtnisses (.110) wurde bereits das volle Quantum an potentieller Varianzaufklärung (.116) erreicht. Auch hier ist demzufolge wieder davon auszugehen, dass jegliche innerhalb des kognitionspsychologischen Regressionsbereichs liegende Varianz innerhalb des Arbeitsgedächtnisbereichs lag. Da die Variablen-Extraktion die Varianz der phonologischen Bewusstheit allerdings wiederum um denselben Betrag (.111) senkte, schien die innerhalb des kognitionspsychologischen Regressionsbereichs befindliche SIN-Varianz nur im Überschneidungsbereich von Arbeitsgedächtnis und phonologischer Bewusstheit zu liegen zu kommen. Mit .068 wurde der Anteil angegeben, der auf den dreifach kognitionspsychologischen Überschneidungsbereich entfiel.

In Tabelle 27 wurde ein Überschneidungsbereich zwischen den EEG-Variablen NP und SIN angedeutet. Dessen erwartete Größenordnung betrug ca. .04 bis .05.. Um dies hier zu prüfen, wurde der Komplex aus allen drei kognitionspsychologischen Konstrukten um deren gemeinsame Varianz bereinigt. Der resultierende Wert (.190) war um .04 kleiner, als die Summe der 
durch die Extraktion der beiden einzelnen EEG-Variablen $(\mathrm{NP}+\mathrm{SIN}=.12+.11)$ bedingten Varianzeinbußen des Arbeitsgedächtnisses. Diese .04 würden sich gut durch Überschneidung erklären lassen und sind hinsichtlich der Größenordnung mit der entsprechenden Erwartung konsistent. Eine Überschneidung zwischen den beiden nichtsprachlichen EEG-Variablen wurde demzufolge angenommen.

In den Erläuterungen zu Tabelle 29 wurde behauptet, dass sämtliche innerhalb des Varianzbereichs der kognitionspsychologischen Konstrukte befindliche EEG-Varianz durch die Varianz des Arbeitsgedächtnisses abgedeckt sei. Dies wurde überprüft, indem die drei EEGVariablen zum einen aus dem Arbeitsgedächtnis und zum anderen aus dem Komplex der kognitionspsychologischen Konstrukte extrahiert wurden. Beide Residuen waren um dasselbe Maß an Varianzaufklärung hinsichtlich der Rechtschreibvarianz beeinträchtigt (.266 bzw. .268).

Dies bestätigte auch die Extraktion der einzelnen EEG-Variablen.

Die Extraktion des PHO und des NP kostete den Komplex der kognitionspsychologischen Variablen beinahe exakt denselben Betrag an Varianzerklärung, den es auch das Arbeitsgedächtnis allein gekostet hatte (und bestätigte somit außerdem deren Größenschätzungen). Der entsprechende Wert für den SIN differierte jedoch von dem, was sich aus der Extraktion aus dem Arbeitsgedächtnis ergeben hatte. Dafür konnte keine plausible Erklärung gefunden werden. Die zu treffende Aussage, nämlich dass alle EEG-Varianz, die innerhalb des Bereichs der kognitionspsychologischen Konstrukte lag, auch innerhalb der Arbeitsgedächtnis-Varianz lag, konnte dennoch gehalten werden, da der Varianzraum, den der SIN im gesamten kognitionspsychologischen Varianzkomplex beanspruchte nicht höher als der Raum lag, den die EEG-Variable innerhalb des Arbeitsgedächtnisses beanspruchte.

Als Größenannahme für die SIN-Varianz wurden trotz des abweichenden Befundes weiterhin die .11 angenommen, da dieser Wert mehrfach das Resultat von Berechnungen war und daher reliabler als eine einmalige Abweichung erschien. Zudem ist in Betracht zu ziehen, dass es sich bei der Abweichung um eine Größenordnung von .013, das heißt lediglich einem Prozent handelte.

Die obigen Ausführungen münden in folgende abschließende Schemaabbildung: 


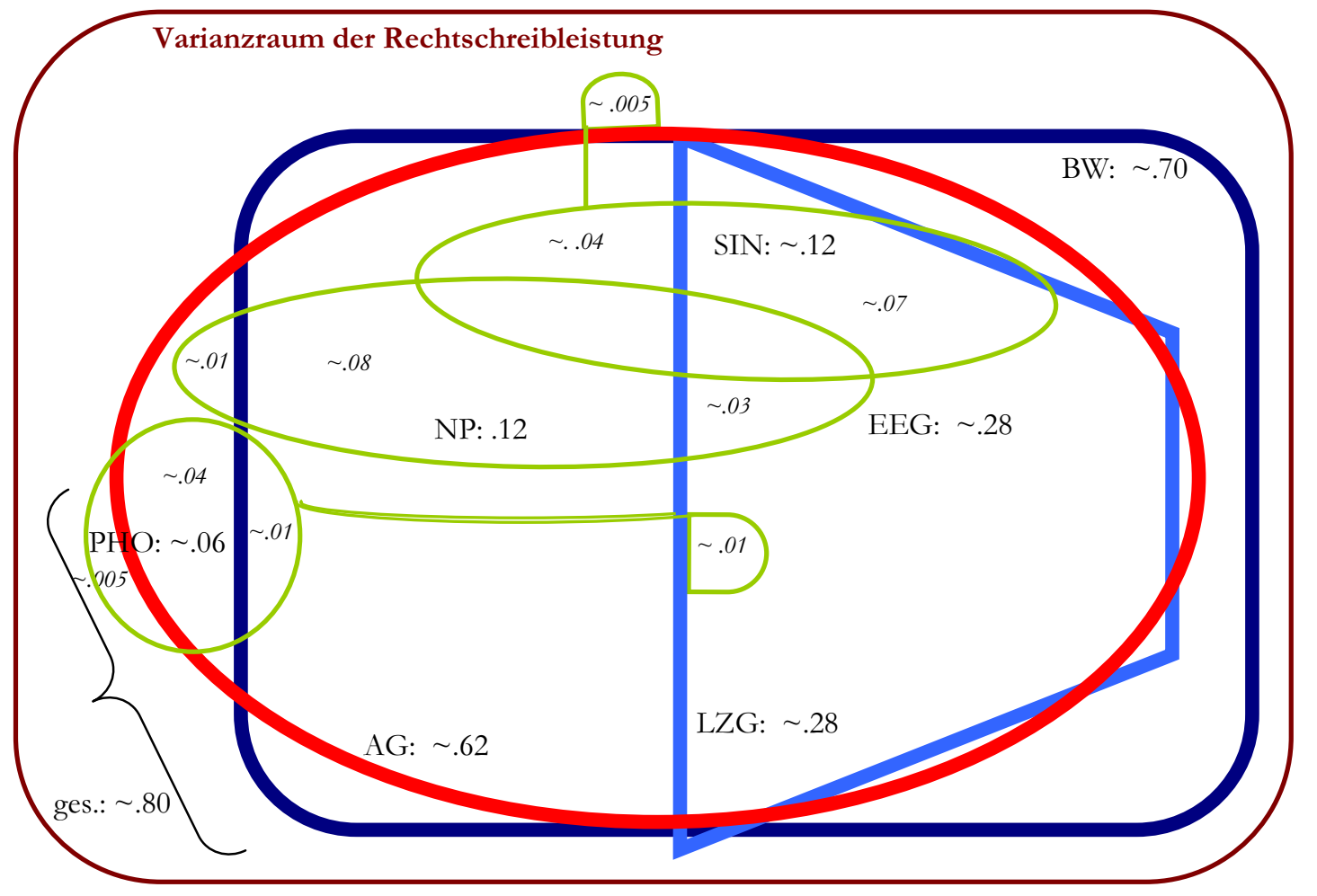

Abb. 17:

Mögliche Varianzstruktur der kognitionspsychologischen Konstrukte und der Ebene der neurophysiologischen Verarbeitung im Varianzraum der Rechtschreibleistung.

Anmerkung: Varianzanteile der Konstrukte bzw. Variablen, die nicht mit der Rechtschreibleistung in Zusammenhang stehen, sind hier nicht abgebildet.

Die gerade gedruckten Zahlen bezeichnen die Höhe der Varianzerklärung $\left(\mathrm{R}^{2}\right)$ eines Konstrukts bzw. einer MMN-Variablen. Kleiner und kursiv gedruckt sind die geschätzten Größenordnungen in verschiedenen Varianzbereichen befindlicher Stimulusanteile.

Lesebeispiel: Insgesamt erklären die Konstrukte und die EEG-Variablen einen Anteil von ca. .8 der Varianz der Rechtschreibleistung. Hiervon erklärt die Variable PHO insgesamt einen ca. .06 großen Anteil. Davon wiederum liegen ca. .005 außerhalb des Bereiches von kognitionspsychologischen Konstrukten erklärter Varianz. Ca. .04 Anteile überschneiden sich lediglich mit dem Varianzbereich des Arbeitsgedächtnisses und ca. .01 Anteil befindet sich in einem Überschneidungsbereich von Arbeitsgedächtnis und phonologischer Bewusstheit. Ein weiterer Anteil eines Ausmaßes von ca. .01 befindet sich in einem Überschneidungsbereich mit allen dreien kognitionspsychologischen Konstrukten.

AG: phonologisches Arbeitsgedächtnis; BW: phonologische Bewusstheit; LZG: Zugriff auf das Langzeitgedächtnis, EEG: Gesamt der drei MMN-Variablen..

PHO: Peaklatenz der MMN der Bedingung PH-G, Segment B; NP: Fläche der MMN der Bedingunng NP-D, Segment B, SIN: Fläche der MMN der Bedingung SIN-D.

\subsubsection{BeURTEILUNG DER EMPIRISCHEN VORHERSAGE $\quad 3 \mathrm{~A} \quad$ UND}

\section{ZUSAMMENFASSUNG DER UNTERSUCHUNGEN ZUM ERKLÄRUNGS-}

\section{POTENZIAL DER KONSTRUKTE / EBENEN}

Den Nachweis gemeinsamer Varianzanteile phonologischer und auditiv sensorischer Verarbeitung im Hinblick auf die Erklärung der Rechtschreibleistung erbrachte der vorangehende Abschnitt 5.3.2.3. mit hoher Eindeutigkeit. Die empirische Vorhersage 3a konnte damit 
bestätigt werden. Desweiteren bestand das Ziel, diesen detektierten Zusammenhang zu spezifizieren, wobei neben der gegenseitigen Abhängigkeit der kognitionspsychologischen Konstrukte insbesondere die Frage im Raum stand, ob die neurophysiologische Ebene Erklärungspotenzial der Rechtschreibleistung bereit stellt, welches durch die kognitionspsychologischen Konstrukte noch nicht erfasst wird. Hierzu lässt sich an dieser Stelle zusammenfassen, dass die bereits starke Varianzerklärung von ca. 80\%, die durch die Gesamtheit der kognitionspsychologischen Konstrukte erbracht wurde, durch Einbezug der EEG-Variablen nicht (nennenswert) gesteigert werden konnte. Additive Komponenten von Seiten der EEGVariablen beliefen sich in der vorliegenden Untersuchung auf ein sehr geringfügiges Ausmaß (Schätzwert: insgesamt ca. 1\%). Der ca. 30\% ausmachende Varianzanteil der EEG-Variablen fiel in Überlappungsbereiche mit allen drei kognitionspsychologischen Variablen, insbesondere aber mit dem phonologischen Arbeitsgedächtnis, welches nahezu alle Varianz abzudecken vermochte, die die EEG-Variablen mit den kognitionspsychologischen Konstrukten teilten.

\subsubsection{MODELLIERUNG DES ZUSAMMENHANGS PHONOLOGISCHER UND AUDITIV SENSORISCHER VERARBEITUNGSDEFIZITE}

Um zu prüfen, ob innerhalb des ermittelten Zusammenhangs zwischen phonologischen und auditiv sensorischen Verarbeitungsdefiziten eine gerichtete Bezogenheit der Komponenten zueinander bestand, wie sie die empirische Vorhersage $3 \mathrm{~b}$ formuliert, wurde ein Modell berechnet. Für diese kausalanalytische Fragestellung erschien die Methode 'LISREL' besonders geeignet (Software: LISREL 8.54, K. Jöreskog \& D. Sörbom).

Die zentrale Größe zur Analyse eines Zusammenhangs in einem LISREL-Modell sind die Pfadkoeffizienten. Diese geben jeweils den Anteil der Standardabweichung wieder, den eine einflussnehmende Größe auf eine andere nimmt, korrigiert um den Einfluss anderer Variablen, die auf die beiden Größen wirken (nach Backhaus, Erichson, Plinke \& Weiber, 1994; S. 359). Der Anteil unerklärter Varianz ergibt sich dann im standardisierten Modell -mit welchem die Berechnungen in der vorliegenden Arbeit angestellt werden- aus der Subtraktion des quadrierten Pfadkoeffizienten von eins.

Für diese Pfadkoeffizienten können t-Werte berechnet werden, die prüfen, ob die Parameter signifikant von Null verschieden sind. Nach den Autoren des Programmes LISREL (K. Jöreskog \& D. Sörbom) ist dies der Fall, wenn ein t-Wert mehr als zwei beträgt. Für die vorliegende 
Fragestellung ist das t-Wert-Kriterium jedoch von untergeordneter Priorität. Es soll stets mit aufgeführt werden, ohne über Annahme oder Ablehnung der Modellanpassung zu entscheiden. Als bedeutender wurden die Polung und die Höhe eines Pfadkoeffizienten selbst angesehen: Da die Vorhersage nicht zwingend starke Pfade fordert, wurde festgelegt, dass ein Pfad dann angenommen werden kann, wenn sein Koeffizient einen positiven Wert über .10 aufweist.

Die Anpassungsqualität eines Modells kann weiterhin durch verschiedene Güteparameter beschrieben werden, die für die Beurteilung eines Modells gemeinsam zu betrachten sind. (Bei der Berechnung eines Modells werden diese von der LISREL-Software automatisch ausgegeben.) In der vorliegenden Arbeit wurden die üblichsten Parameter herangezogen, nämlich der $\chi^{2}$-Wert, der Goodness-of-Fit-Index (GFI), der Adjusted-Goodness-of-Fit-Index (AGFI) sowie das Root-Mean-Square-Residual (RMR). Der $\chi^{2}$-Test prüft die Alternativhypothese, dass die vorliegende Kovarianzmatrix einem beliebigen anderen Modell entspricht. In der Praxis werden meist diejenigen Modelle verworfen, bei welchen der zugehörige p-Wert unter .10 liegt. Weiterhin gelten $\chi^{2}$-Werte, die kleiner oder in ähnlicher Größenordnung wie die Anzahl der Freiheitsgrade liegen, als gut.

Der GFI misst die relative Menge an Varianz und Kovarianz, der das Modell insgesamt Rechnung trägt und kann daher Werte zwischen null und eins annehmen. Werte ab .90 und besonders $\mathrm{ab} .95$ kennzeichnen gute Modellanpassungen. Im Nenner des AGFI sind zusätzlich die Freiheitsgrade berücksichtigt; er belohnt demzufolge die Sparsamkeit eines Modells. Auch für diesen Parameter gilt, dass die Modellanpassung als umso besser zu erachten ist, je mehr er sich dem Wert eins nähert. In der Regel liegt der AGFI unter dem GFI.

Der RMR bezieht sich auf die in einem Modell nicht erklärbaren Residualvarianzen. Die Anpassungsgüte ist demzufolge umso besser, je näher der RMR bei null liegt.

Für die Beurteilung des Modells sollten in der vorliegenden Studie die üblichen Kriterien gelten, was bedeutet, dass der $\chi^{2}$-Wert kleiner oder gleich der Anzahl Freiheitsgrade sein und der entsprechende p-Wert über .10 liegen sollte. Ein optimaler GFI zwischen .95 und 1.00 war jedoch nicht zu erwarten, da aufgrund der inhaltlichen Nähe der Konstrukte die Messvariablen innerhalb einer latenten Variable sowie $\mathrm{zu}$ Messvariablen anderer latenter Variablen Korrelationen aufwiesen. (Diese wurden in der Berechnung, um eine artifizielle Anpassungsqualität zu vermeiden, nicht korrigiert.) Als Minimum für den GFI wurde daher der Wert .90 festgelegt; der AGFI sollte .80 nicht unterschreiten. Das Kriterium für die Residualvarianzen wurde mit einer Akzeptanz von maximal 100 demzufolge ebenfalls moderat formuliert. 
Die Besonderheit des LISREL-Ansatzes besteht in der Möglichkeit, gemessene Variablen (,Messvariablen“) zu nicht messbaren, diese aber inhaltlich verbindenden „latenten Variablen“ zusammenzufassen. Da die die in den vorangegangenen Abschnitten erfolgte Analyse des Zusammenhangs im Folgenden weiter fortgesetzt wurde, kamen für die Berechnung des Modells keine anderen als die in Punkt 5.3.2.1. konstruktspezifisch aggregierten Variablen in Betracht. Jedes kognitionspsychologische Konstrukt wurde im LISREL-Modell als latente Variable konzipiert, welche durch die ihr zugehörigen aggregierten Variablen erklärt wurde. Um eine höhere Auflösung im EEG-Bereich zu gewährleisten, wurde jede der drei EEGVariablen als jeweils eine eigene latente Variable gehandhabt. Das entsprach ebenfalls dem Vorgehen in Punkt 5.3.2.3.

Ausgangspunkt des LISREL-Modells war eine Kovarianzmatrix, die im Programm SPSS 10 erstellt wurde und in Anhang C einzusehen ist. Aufgrund ihrer relativ geringen Werte wäre es zu überlegen gewesen, die Interaktionsvariable des Kunstwort-Nachsprechens aus dem Messvariablenagglomerat für das Arbeitsgedächtnis herauszulösen. Im Hinblick auf die bereits genannte inhaltliche Abhängigkeit der Analyseschritte wurde auf diese Modifikation jedoch verzichtet. (Hierbei ist anzumerken, dass eine Prüfberechnung nach Ausschluss der Interaktionsvariablen keine anderen Resultate erzeugte, als es unter Einbezug der Variablen der Fall war.)

Um das Informationspotenzial der Daten maximal auszuschöpfen, sollte das ModellprüfVerfahren schrittweise erfolgen: Zunächst war zu prüfen, inwieweit sich die kognitionspsychologischen Konstrukte in die postulierte Ordnung bringen ließen. Erst daraufhin wurde die EEG-Ebene eingefügt. So ließen sich die Auswirkungen des Einfügens der EEG-Variablen auf die Interaktion der kognitionspsychologischen Konstrukte untereinander verfolgen. Weiterhin sollte im Falle, dass das postulierte Modell den Prüfkriterien nicht standhalten sollte, exploriert werden, ob eine besser angepasste Lösung möglich wäre. Diese Schritte werden in den folgenden Abschnitten dokumentiert. 


\subsubsection{PRÜFUNG DER MODELLSTRUKTUR GEMÄß DER EMPIRISCHEN VORHERSAGE 3B}

\subsection{STRUKTUR DER KOGNITIONSPSYCHOLOGISCHEN KONSTRUKTE}

Werden die kognitionspsychologischen Konstrukte in die vorhersagegemäße Modellstruktur gefasst, entsteht die folgende Anpassung:

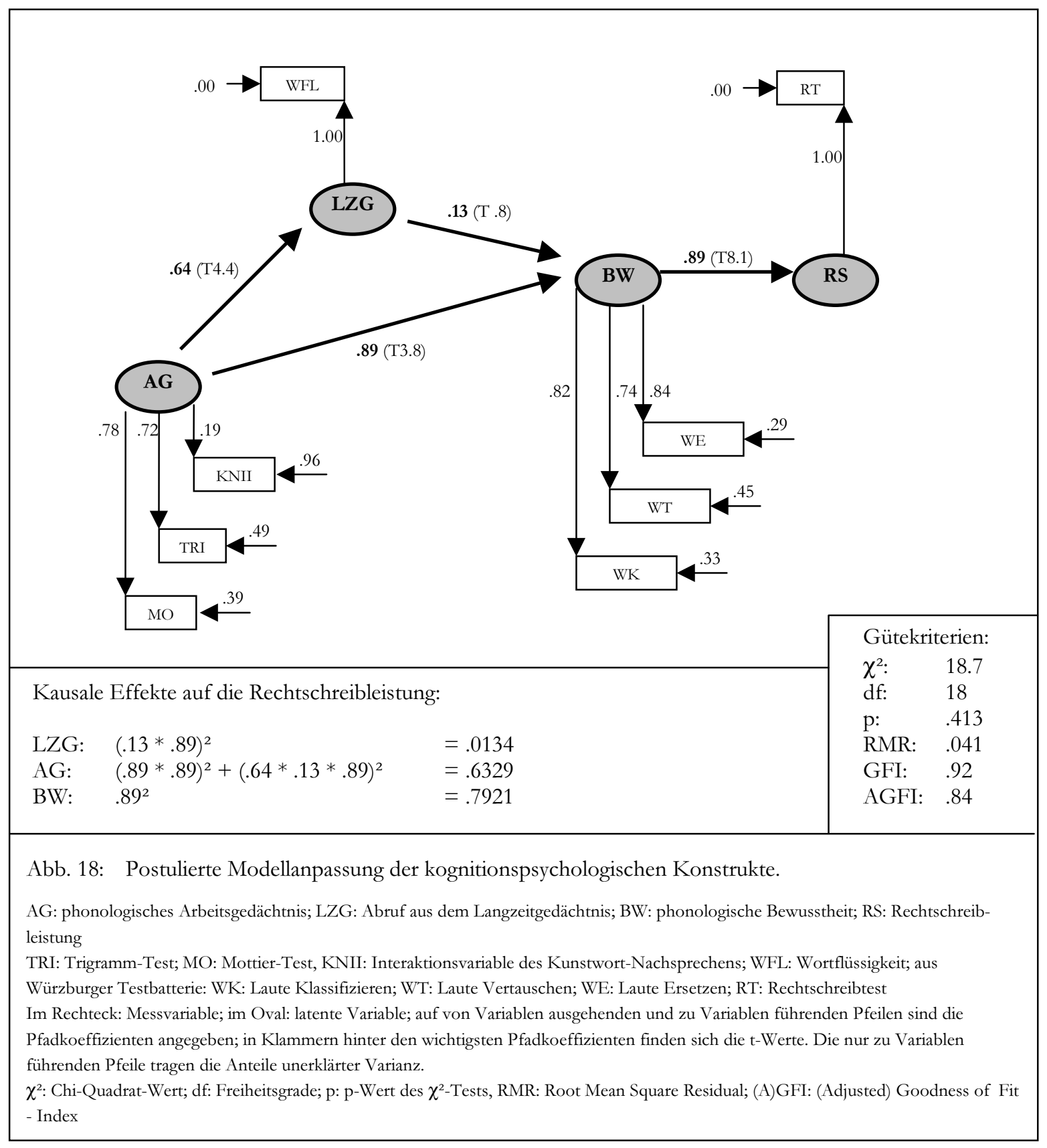


Sämtliche Pfadkoeffizienten dieser Lösung trugen ein positives Vorzeichen und wiesen Werte über .1 auf. Mit Ausnahme des Pfades vom Abruf aus dem Langzeitgedächtnis auf die phonologische Bewusstheit waren es starke Pfade, die auch das Signifikanzkriterium erfüllen könnten.

Die Gütekriterien zeichneten dieses Modell zwar nicht als außergewöhnlich gut, aber als akzeptabel aus. Extrem gute Anpassungen wurden aufgrund der hohen Multikollinearität in den Daten nicht erwartet.

Die Rangfolge der resultierenden kausalen Effekte der Konstrukte auf die Rechtschreibleistung war dieselbe, die auch die Varianzschätzungen der multiplen Regression erbracht hatten. Die Größenordnung des Kausaleffektes war nur bezüglich des Arbeitsgedächtnisses mit den Varianzschätzungen in Einklang zu bringen $\left(\mathrm{R}^{2}: 623\right.$; kausaler Effekt: .633). Der kausale Effekt (.792) der phonologischen Bewusstheit wurde im Vergleich zum Regressionswert (.696) etwas überschätzt; der Einfluss des Langzeitgedächtnis hingegen wurde im Modell mit .013 vergleichsweise $\left(\mathrm{R}^{2}: 283\right)$ gering eingeschätzt. Führt man sich vor Augen, dass die Verbindung zwischen Arbeitsgedächtnis und Abruf aus dem Langzeitgedächtnis auch eher interaktiv als gerichtet vorstellbar sein kann, und der so in einem Modell ein Doppelpfeil möglicherweise gerechter würde, so resultiert eine gewisse Toleranz gegenüber Abweichungen in den Angaben für kausale Effekte bzw. Regressionskoeffizienten. Derartige Vergleiche sollen hier ohnehin nicht überstrapaziert werden, da identische Größenordnungen nicht erwartet werden können, da die multiple Regression für jedes Konstrukt separat erfolgte, die Konstrukte im LISRELModell jedoch innerhalb bestimmter Zusammenhänge geprüft wurden; sie seien hier lediglich richtlinienartig aufgeführt. Eine eventuelle interaktive Beziehung zwischen Arbeitsgedächtnis und Abruf aus dem Langzeitgedächtnis kann aus software-technischen Gründen erst nach dem Einfügen der EEG-Variablen, wenn das Arbeitsgedächtnis nicht mehr den Beginn der Vorhersagekette darstellt, (interessehalber) getestet werden.

Zusammenfassend betrachtet, schien das Modell der kognitionspsychologischen Konstrukte ausreichend valide, um es wie vorgesehen um die EEG-Variablen zu erweitern. 


\subsection{EINFÜGEN DER EEG-VARIABLEN}

Gemäß der Hypothese sollte die optimale Position für das Einfügen der EEG-Variablen vor dem Arbeitsgedächtnis sein. Wenn die Ergebnisse der multiplen Regression Anlass zur Vermutung geben, der MMN-Parameter erkläre relativ zu den kognitionspsychologischen Konstrukten zusätzliche Rechtschreibvarianz, bestünde eine weitere Möglichkeit in einem direkten Einfluss auf die Rechtschreibleistung. Auch wenn das vorige Kapitel allenfalls auf einen geringen direkten Einfluss der elektrophysiologischen Variablen auf die Rechtschreibung deutet, sollte dies ausreichen, um diese Möglichkeit einzuräumen.

Zur Prüfung des Modells wurden nicht einfach die EEG-Variablen vor das Arbeitsgedächtnis und vor die Rechtschreibleistung gestellt. Um nebenbei zu erfahren, wie sich der Einfluss der EEG-Variablen auch auf die anderen Konstrukte verhielt, wurden in einem Zwischenschritt Pfade aller MMN-Variablen auf alle kognitionspsychologischen Konstrukte zugelassen. Diese Zwischenlösung (nach Elimination der negativen Pfade und der Pfade mit Nullwerten) ist in Abbildung 19 dargestellt. Sukzessive wurden jeweils die geringsten Pfade, das heißt diejenigen, deren Koeffizienten unter .10 blieben, eliminiert. Das entsprechende resultierende Modell stellt Abbildung 20 dar.

An diesem Modell wurde zusätzlich die Auswirkung eines rückwirkenden Pfades vom Abruf aus dem Langzeitgedächtnis hin zum Arbeitsgedächtnis erprobt: Der Pfadkoeffizent betrug lediglich .08 und konnte demzufolge vorhersagegemäß unberücksichtigt bleiben. 


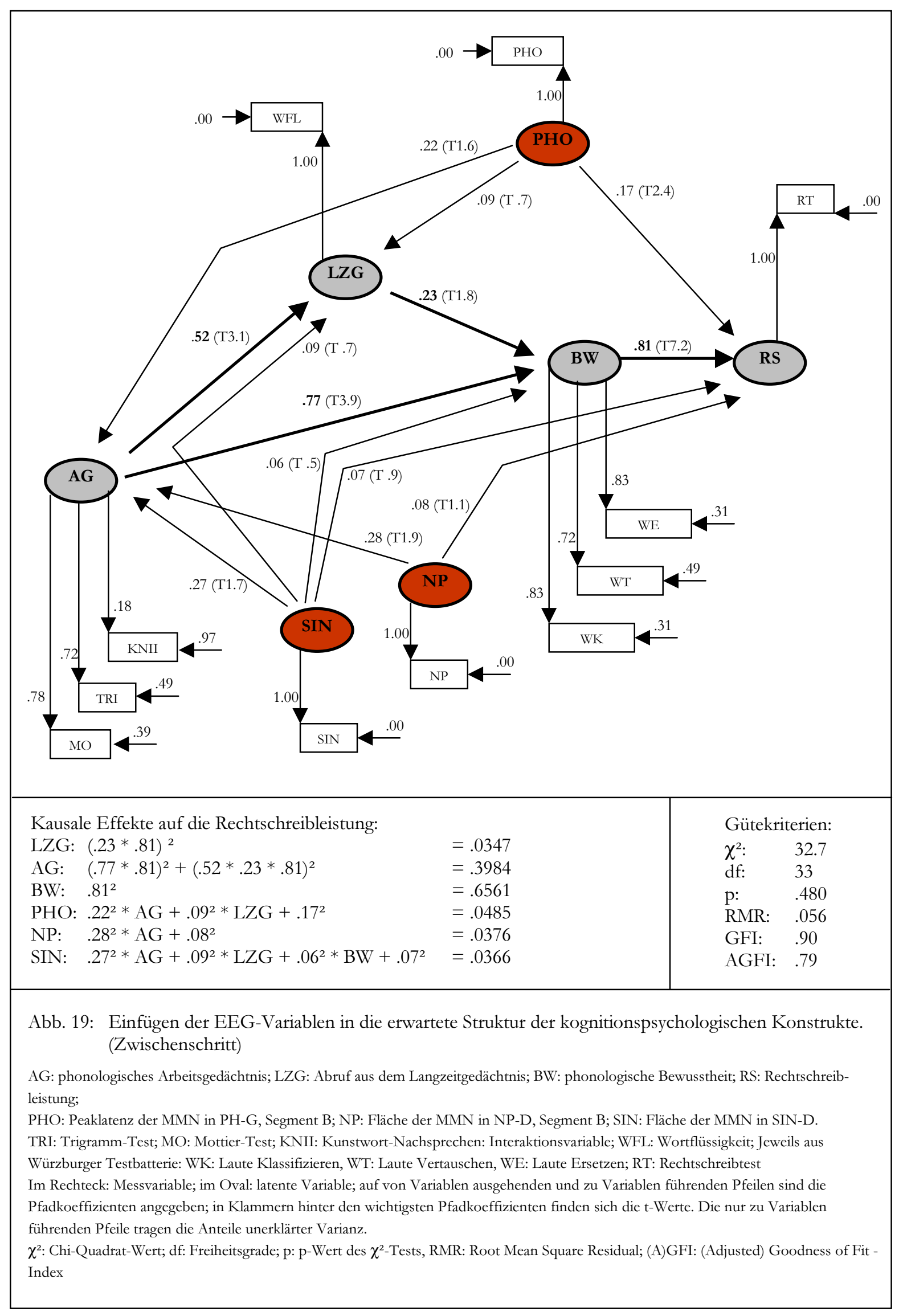




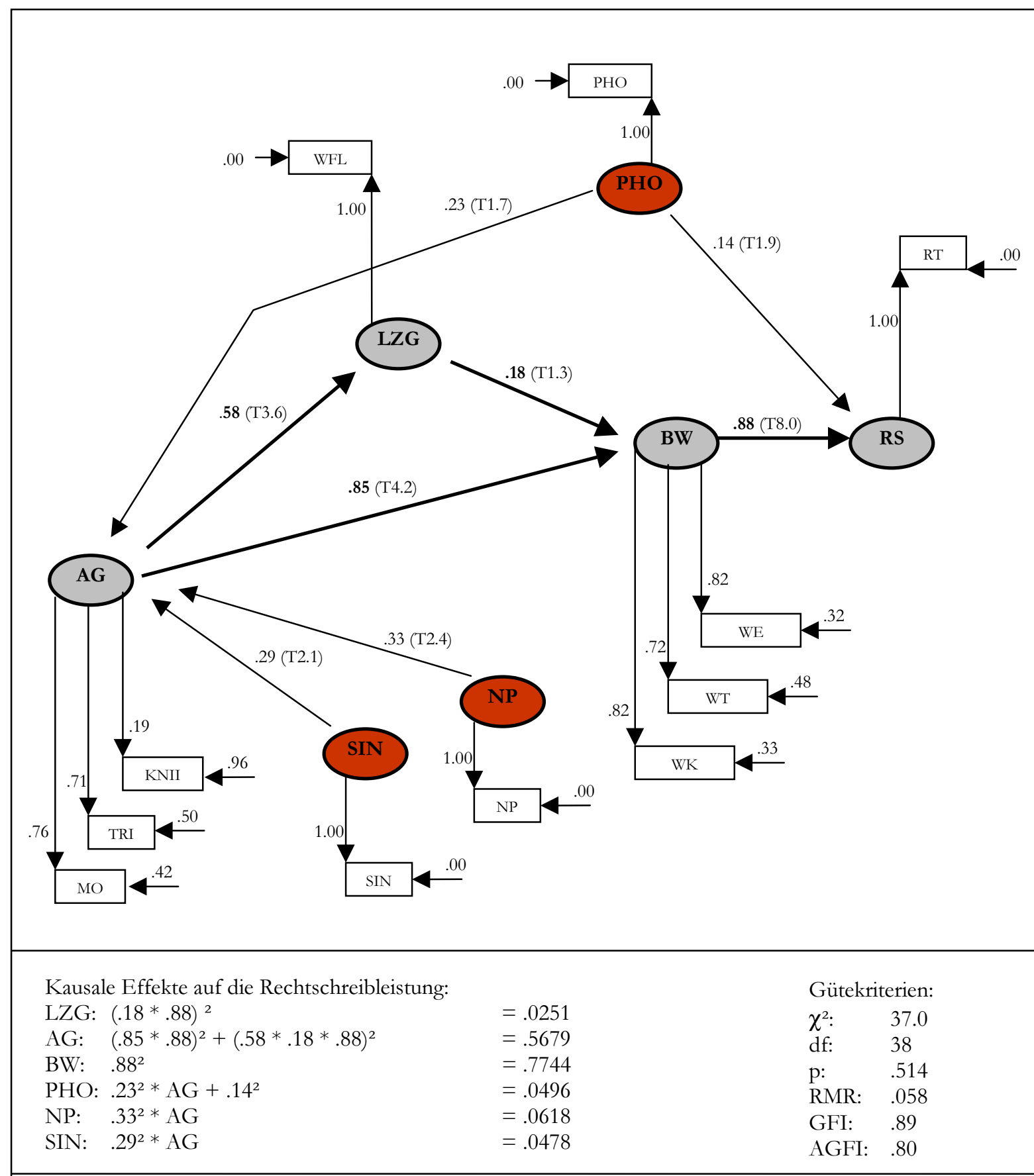

Abb. 20: Bereinigung von weniger bedeutenden Pfaden nach Einfügen der EEG-Variablen in die Struktur der kognitionspsychologischen Konstrukte.

AG: phonologisches Arbeitsgedächtnis; LZG: Abruf aus dem Langzeitgedächtnis; BW: phonologische Bewusstheit; RS: Rechtschreibleistung;

PHO: Peaklatenz der MMN in PH-G, Segment B; NP: Fläche der MMN in NP-D, Segment B; SIN: Fläche der MMN in SIN-D. TRI: Trigramm-Test; MO: Mottier-Test; KNII: Kunstwort-Nachsprechen: Interaktionsvariable; WFL: Wortflüssigkeit; Jeweils aus Würzburger Testbatterie: WK: Laute Klassifizieren, WT: Laute Vertauschen, WE: Laute Ersetzen; RT: Rechtschreibtest.

Im Rechteck: Messvariable; im Oval: latente Variable; auf von Variablen ausgehenden und zu Variablen führenden Pfeilen sind die Pfadkoeffizienten angegeben; in Klammern hinter den wichtigsten Pfadkoeffizienten finden sich die t-Werte. Die nur zu Variablen führenden Pfeile tragen die Anteile unerklärter Varianz.

$\chi^{2}$ : Chi-Quadrat-Wert; df: Freiheitsgrade; p: p-Wert des $\chi^{2}$-Tests, RMR: Root Mean Square Residual; (A)GFI: (Adjusted) Goodness of Fit Index 
Dabei wurde deutlich, dass trotz des Zulassens aller möglichen von den EEG-Variablen ausgehenden Pfade lediglich solche übrig blieben, die auf das Arbeitsgedächtnis bzw. auf die Rechtschreibleistung gerichtet waren. Dies war vorhersagegemäß. Auf das Arbeitsgedächtnis richteten sich die Pfade aller drei EEG-Variablen; für die Rechtschreibleistung konnte ein kleiner Einfluss der Variable PHO verzeichnet werden.

Allerdings waren diese Pfade nicht allzu varianzstark: die Koeffizienten bewegten sich zwischen .14 und .33 (und unter- oder überschritten ein potentielles Signifikanzkriterium jeweils knapp). Auf die Beziehungen innerhalb der kognitionspsychologischen Konstrukte zeigte das Einfügen der EEG-Variablen kaum Auswirkungen. Die kausalen Effekte der Konstrukte bewegten sich weiterhin in etwa in dem Rahmen, der vom Modell ohne die EEG-Variablen gesetzt worden war. Für die MMN-Variablen wurden kausale Effekte zwischen knapp fünf und sechs Prozent (PHO: .050; NP: .062; SIN: .048) berechnet. Diese Werte differierten wie die der kognitionspsychologischen Konstrukte von den entsprechenden Schätzungen der multiplen Regression (PHO: .061; NP: .117; SIN: .116). Die Differenzen sind jedoch nicht so drastisch ausgeprägt, dass sie das Modell falsifizieren würden. Gemäß der meisten Gütekriterien wäre eine Annahme des Modells gerechtfertigt. Der Index GFI allerdings unterschritt den kritischen Wert von .90 um .01 und zeigte damit an, dass die Modellanpassung nicht mehr akzeptabel war. Das Modell musste daher abgelehnt werden.

Da zwischen den beiden dynamischen Deviant-Bedingungen gemeinsame Varianz bestand (siehe Punkt 5.3.2.3.2.), wurde der Versuch unternommen, diese beiden zu einer latenten Variable (NPSIN) zusammenzufassen (vgl. Abb. 21). Der Anpassungsindex GFI sank hierbei auf .88, so dass auch diese Modellvariante nicht akzeptiert werden konnte. Allerdings war bei dieser Lösung zu bemerken, dass der kausale Effekt der gemeinsamen EEG-Variable den der beiden einzelnen um ein Vielfaches überstieg (.401). 


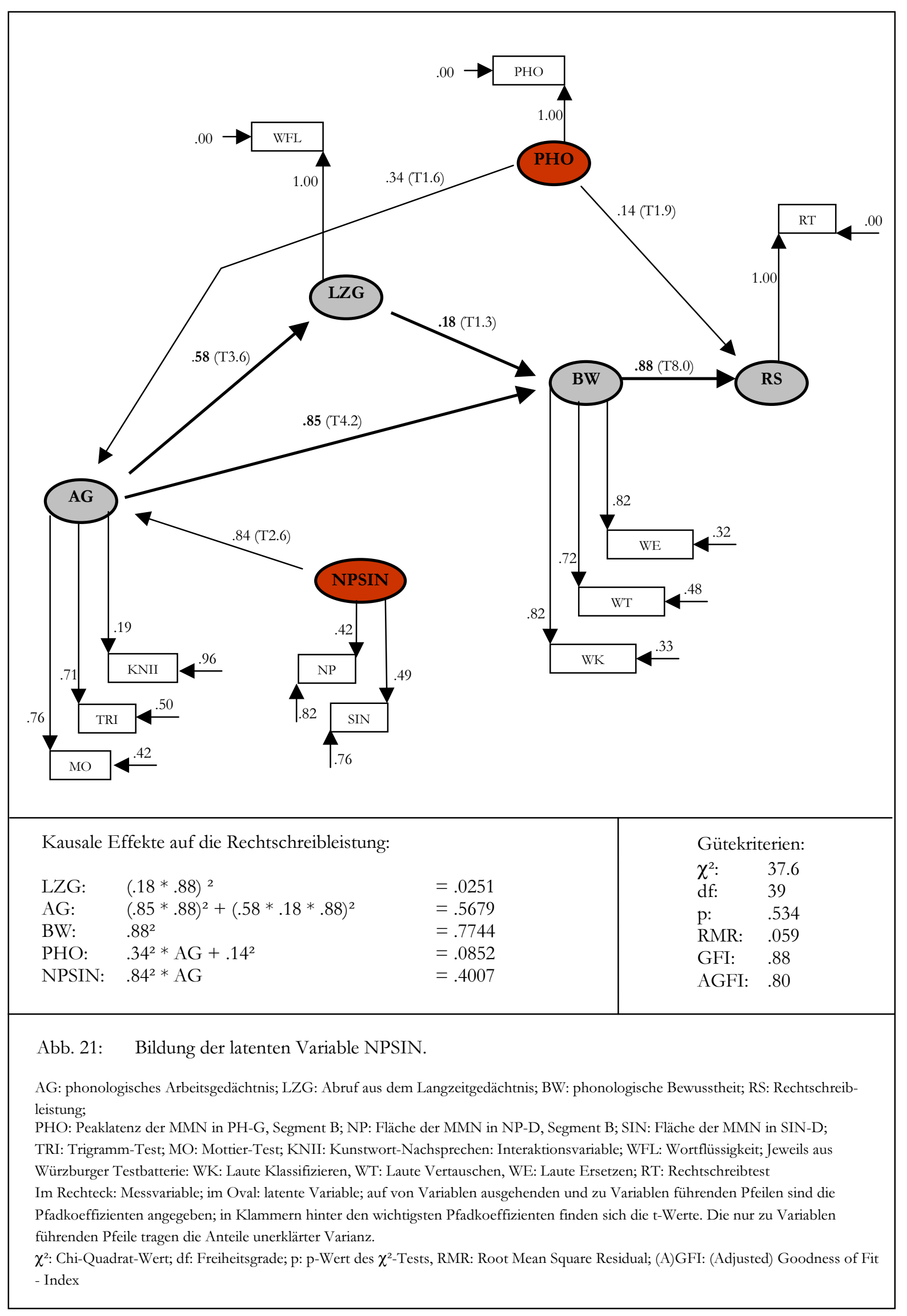




\subsubsection{EINE MODELL-AlternAtive}

\subsection{STRUKTUR DER KOGNITIONSPSYCHOLOGISCHEN KONSTRUKTE}

Nachdem das Modell in Abbildung 21 durch das Einfügen aller EEG-Variablen an allen Orten und dem sukzessiven Herauslösen der wenig varianztragenden Elemente etabliert wurde, kann der Grund für die schlechte Anpassung nicht in einem ungeschickten Einfügen der Variablen liegen. $\mathrm{Zu}$ überlegen wäre jedoch, ob eventuell ein anderes Grundmodell bessere Anpassungsindizes erreichen könnte. Dies wurde auf zweierlei Wegen versucht. Zum einen wurden die jeweils vorhersagekräftigste(n) Komponente(n) eines Konstrukts sukzessive selegiert, für die wiederum die jeweils vorhersagekräftigste(n) Komponente(n) ausgewählt wurden etc. Ein zweiter Weg bestand im simplen Durchprobieren der möglichen Anordnungen und anschließendem Vergleich der Passungskriterien. Beide Wege führten zu keiner anderen Lösung als der postulierten und oben geprüften. Allerdings bestand die Möglichkeit, zwei weitere Pfade einzufügen, denen bislang nicht die Hauptaufmerksamkeit gegolten hatte. Es handelte sich um die direkten Vorhersagen jeweils des Abrufs aus dem Langzeitgedächtnis und des Arbeitsgedächtnisses auf die Rechtschreibleistung. Das resultierende Modell (6-Pfad-Modell) ist in Abbildung $22 \mathrm{zu}$ sehen. 


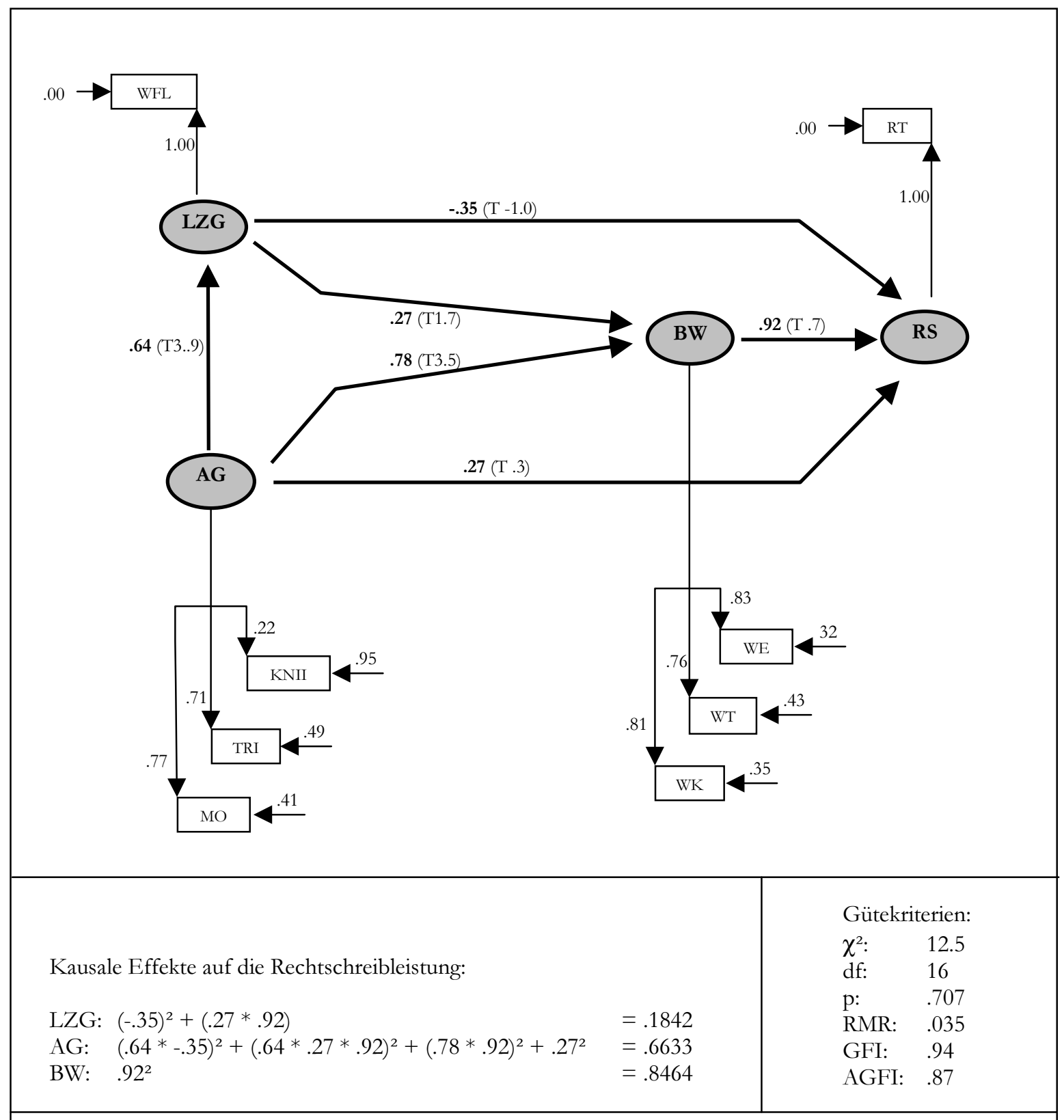

Abb. 22: Alternative Modell-Lösung der kognitionspsychologischen Konstrukte.

AG: phonologisches Arbeitsgedächtnis; LZG: Abruf aus dem Langzeitgedächtnis; BW: phonologische Bewusstheit; RS: Rechtschreibleistung

TRI: Trigramm-Test; MO: Mottier-Test, KNII: Interaktionsvariable des Kunstwort-Nachsprechens; WFL: Wortflüssigkeit; aus Würzburger Testbatterie: WK: Laute Klassifizieren; WT: Laute Vertauschen; WE: Laute Ersetzen; RT: Rechtschreibtest Im Rechteck: Messvariable; im Oval: latente Variable; auf von Variablen ausgehenden und zu Variablen führenden Pfeilen sind die Pfadkoeffizienten angegeben; in Klammern hinter den wichtigsten Pfadkoeffizienten finden sich die t-Werte. Die nur zu Variablen führenden Pfeile tragen die Anteile unerklärter Varianz.

$\chi^{2}$ : Chi-Quadrat-Wert; df: Freiheitsgrade; p: p-Wert des $\chi^{2}$-Tests, RMR: Root Mean Square Residual; (A)GFI: (Adjusted) Goodness of Fit - Index 
Für die kausalen Effekte traf wieder zu, dass die Rangfolge der Größenordnungen dieselbe war, wie sie auch im multiplen Regressionsverfahren geschätzt wurde. Der Einfluss der phonologischen Bewusstheit (.846) wurde hier noch höher als im 4-Pfad-Grundmodell angegeben; die Schätzung für das Arbeitsgedächtnis deckte sich in etwa mit der aus Punkt 5.3.2.1., ebenso wie das auch im postulierten Grundmodell der Fall war. Die Angaben für den Gesamteffekt des Abrufs aus dem Langzeitgedächtnis stiegen durch das Hinzufügen eines direkten Pfades auf mehr als das Zehnfache ihres Wertes im 4-Pfad-Grundmodell: Die Schätzung belief sich nun auf .122.

Die Gütekriterien der 6-Pfad-Variante bescheinigten dieser in der Tat eine bessere Anpassung (z.B.: GFI $=.94$, AGFI $=.87)$ als der 4-Pfad-Variante.

Eine eingehendere Betrachtung der Einzelheiten verdeutlichte jedoch, dass die höheren Güteindizes zwar die größeren Anteile erklärter Varianz, möglicherweise jedoch nicht unbedingt eine bessere Qualität der Anpassung anzeigten.

Die Größe der Pfadkoeffizienten, die bereits im postulierten Modell (4-Pfad-Modell) vorkamen, veränderte sich nicht drastisch. Auffallend war jedoch, dass der Vorhersagekoeffizient der phonologischen Bewusstheit, der einen Wert von .92 aufwies, ein potentielles Signifikanzkriterium dennoch weit verfehlte. Der Betrag der beiden neu eingefügten Pfade belief sich auf jeweils circa .3. Diese geringe Größe zeigte, dass die Pfade im Vergleich zu den (meisten der) bereits im 4-Pfad-Modell postulierten Pfaden in der Tat weniger bedeutsam waren. Dies unterstützte damit im Nachhinein die Entscheidung, die zusätzlichen Pfade in der Vorhersage nicht aufzuführen. Weiterhin fiel ins Auge, dass einer dieser beiden, der Koeffizient des Abrufs aus dem Langzeitgedächtnis, ein negatives Vorzeichen trug. In der Matrix ist an dieser Stelle jedoch keine negative Kovarianz zu finden. Es musste sich bei der Polung infolgedessen um ein Artefakt handeln, das möglicherweise durch die hohe Multikollinearität in den Daten zustande gekommen war.

\subsection{EINFÜGEN DER EEG-VARIABLEN}

Um die Betrachtung dieser Alternative zu Ende zu führen und gegebenenfalls Vergleiche anstellen zu können, wurden trotz der Mängel dieser Modelllösung dieselben weiterführenden Schritte wie mit dem 4-Pfad-Modell unternommen. Die entsprechenden Modelle sind in den Abbildungen 23 und 24 dargestellt. 


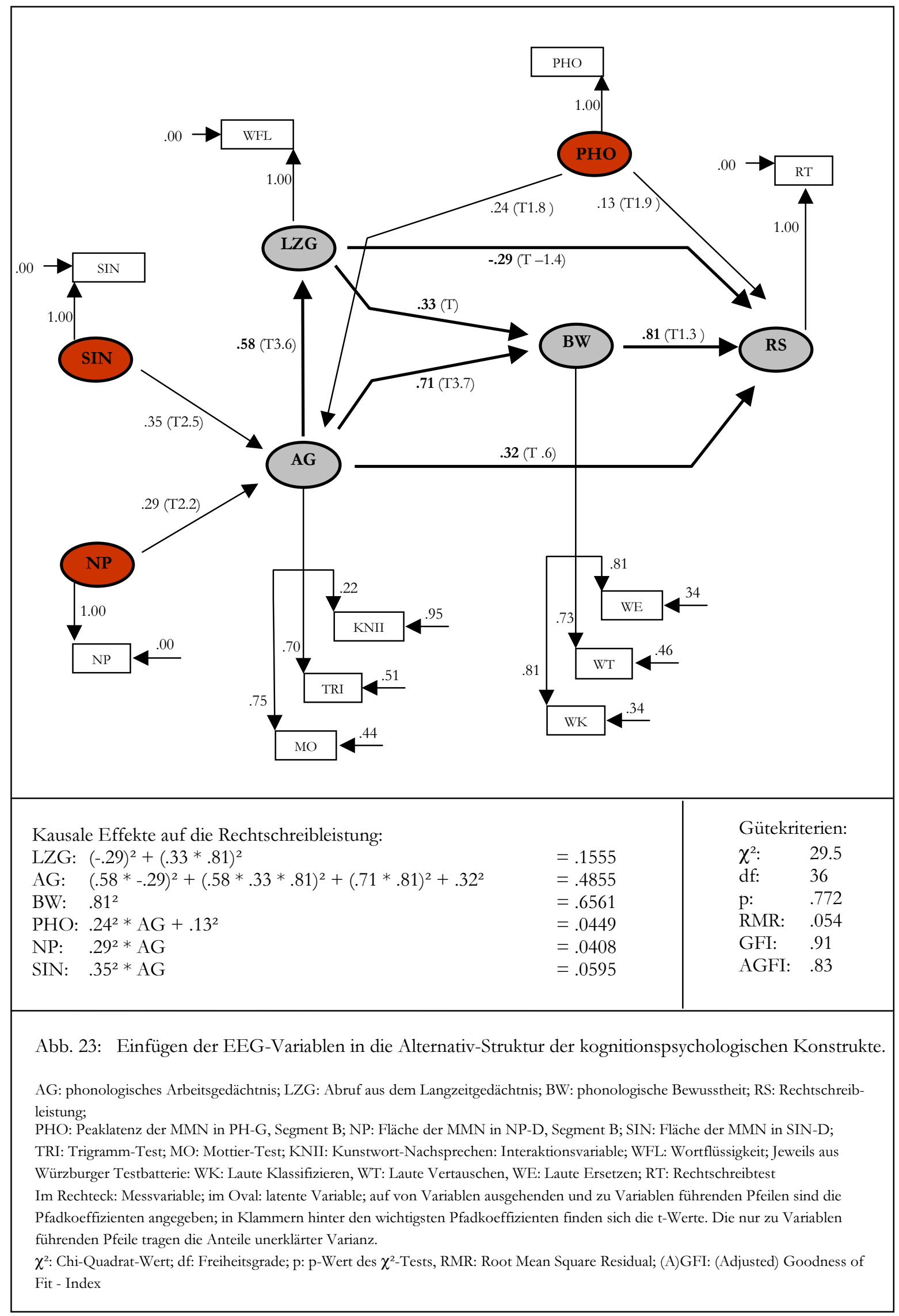




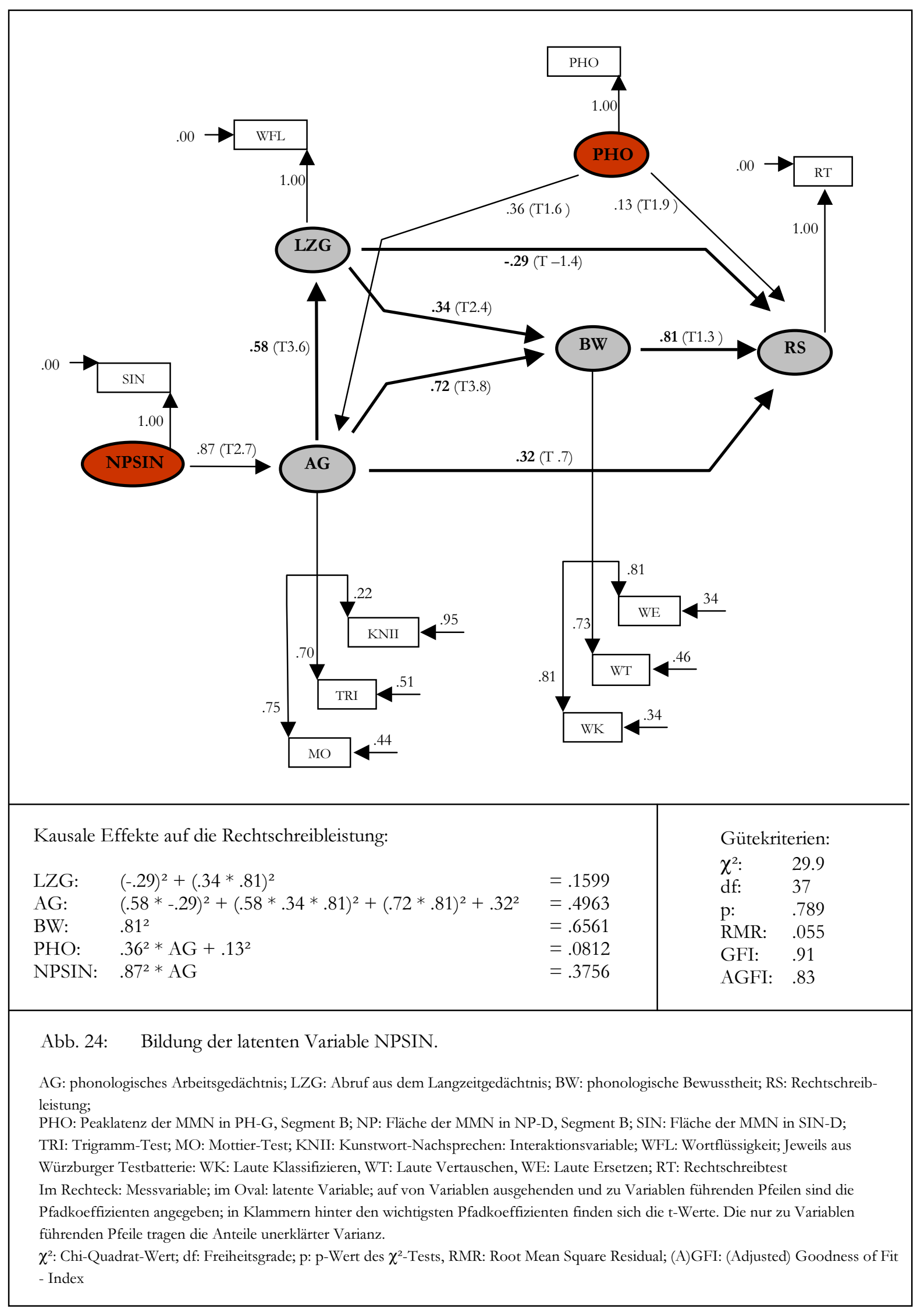


Das Einfügen der EEG-Variablen hatte wie im 4-Pfad-Modell keine nennenswerten Änderungen in der Konstellation der kognitionspsychologischen Konstrukte zur Folge. Desweiteren wurde die Positionierung der EEG-Variablen reproduziert. Die Zusammenfassung der Variablen SIN und NP hatte ebenfalls denselben Effekt wie auch schon im 4-Pfad-Modell.

Der einzige Unterschied zu den Modifikationen des vorhersagegemäßen Modells bestand darin, dass hier die Gütekriterien auch die beiden die EEG-Variablen enthaltenen Varianten als annehmbar auszeichneten. Keine der Modelliösungen könnte jedoch angenommen werden, da eingangs keine entsprechende Vorhersage formuliert wurde. Der negative Pfadkoeffizient würde zudem seinerseits zur Ablehnung dieser Modelle führen.

\subsubsection{BEURTEILUNG DER EMPIRISCHEN VORHERSAGE 3B UND ZUSAM- MENFASSUNG DER MODELL-PRÜFUNG}

Die kognitionspsychologischen Konstrukte erwiesen sich in der vorhersagegemäßen Konstellation in einer Struktur, die zum einen die geforderten Kriterien für Annehmbarkeit erfüllte und der zum zweiten keine sinnvolle Alternative mit besseren Anpassungskriterien gegenüberstand. Die EEG-Variablen fügten sich an den postulierten Positionen ein. Die Hinzunahme der EEGVariablen erhöhte jedoch die Varianz im System um einen größeren Betrag, als die Variablen $\mathrm{zu}$ erklären vermochten. Aus diesem Grunde fiel der Goodness of Fit - Index unter den kritischen Wert und machte damit das Modell bzw. die empirische Vorhersage $3 b$ unannehmbar.

Die resultierende Situation ist ein Dilemma, da angenommene Pfade in einer postulierten Struktur deutlich sichtbar waren und dazu durch die Reproduktion im 6-Pfad-Modell bestätigt wurden, aber Teil eines Modells waren, das als Ganzes nicht angenommen werden konnte.

Als immer wieder auftretende Besonderheit war zu vermerken, dass das Zusammenfassen der beiden EEG-Variablen SIN und NP zu einer latenten Variablen deren Pfadstärke bzw. deren kausalen Effekt drastisch steigerte. 


\subsubsection{ZUSAMMENFASSUNG DER ANALYSEN DES ZUSAMMENHANGS AUDITIV SENSORISCHER UND PHONOLOGISCHER VERARBEITUNGS- DEFIZITE BEI LESE-/RECHTSCHREIBSCHWACHEN KINDERN}

Nach den weitläufigen Analysen bezüglich des Zusammenhangs auditiv sensorischer und phonologischer Verarbeitungsdefizite bei lese-/rechtschreibschwachen Kindern lässt sich Folgendes festhalten: Das Vorhandensein eines Zusammenhangs, das heißt das Vorhandensein gemeinsamer Varianzbereiche der Verarbeitungsebenen bei der Vorhersage der Rechtschreibleistung konnte deutlich nachgewiesen werden. Die Überschneidungen der Varianzbereiche waren stark ausgeprägt; die Anteile nicht geteilter Varianz, den eine EEG-Variable / ein Konstrukt zu erklären in der Lage war, war -wenn existent- stets ein kleinerer Bruchteil der insgesamt von der Variable / dem Konstrukt erklärten Varianz. Von den EEG-Variablen wurde keine Varianzerklärung bereitgestellt, die durch die Varianzerklärung von seiten der kognitionspsychologischen Konstrukte bei der Vorhersage der Rechtschreibleistung noch nicht abgedeckt wären.

Leider konnte eine vermutete Abfolge von Einflussnahmen der Komponenten von der physiologischen Ebene bis hin zum Phänotyp der Rechtschreibleistung nicht nachgewiesen werden. Bei diesen Ergebnissen resultierte das Dilemma, dass die vermutete Abfolge durchaus in Übereinstimmung mit den Daten der vorliegenden Studie erschien, das entsprechende Modell jedoch nicht angenommen werden konnte, da es den Prüfkriterien nicht standgehalten hatte. 


\section{DISKUSSION}

\subsection{DISKUSSION DER BEFUNDE ZUR PHONOLOGISCHEN VERARBEITUNG}

\subsubsection{PSYCHOLOGISCHE HYPOTHESEN}

Bei keinem einzigen kognitionspsychologischen Test blieben die erwarteten signifikanten Gruppenunterschiede zu Ungunsten der lese-/rechtschreibschwachen Gruppe aus. Sie erwiesen sich zudem als effektstark.

Die Tests zum Arbeitsgedächtnis deckten ein geringeres Speichervolumen (Mottier-Test, Kunstwort-Nachsprechen), eine verminderte Verarbeitungspräzision (Interaktionseffekt Gruppe*Silbenanzahl beim Kunstwort-Nachsprechen) des Speichers und eine langsamere Rehearsalgeschwindigkeit (Trigramm-Test) der phonologischen Schleife lese-/rechtschreibschwacher Probanden auf. In Konsistenz hiermit fielen auch deren Indikatorvariablen zur funktionalen Gesamtkapazität der Schleife (Wortspanne, Zahlenspannen) schwächer als die der Kontrollgruppe aus. Damit war die empirische Vorhersage 1.a bestätigt worden.

Der Nachweis verminderter Speicherpräzision war durch die Reproduktion eines an Dysphasikern erhobenen Befundes von Hasselhorn (Hasselhorn et al., 1995; Hasselhorn, 1999; vgl. Grube et al.) mit den lese-/rechtschreibschwachen Kindern erbracht worden: Paradoxerweise zeigten lese-/rechtschreibschwache Kinder im Gegensatz zu Kontrollkindern keine Beeinträchtigung bei der Nachsprechleistung zweisilbiger Kunstworte, wenn diese verzerrt wurden. In dieser Studie zeigten die lese-/rechtschreibschwachen Probanden sogar einen vorübergehenden Leistungsvorteil. Obschon es sich um einen Leistungsvorteil handelte, wurde hinter diesem Phänomen eine Minderleistung des phonetischen Speichers vermutet und der Effekt dahingehend interpretiert, dass lese-/rechtschreibschwache Kinder an eine unpräzise Hörwahrnehmung gewöhnt sein mögen und daher bei der Wiedergabe real unpräzise dargebotener Pseudoworte im Vorteil wären, solange diese Pseudoworte nicht länger sind als ihr Speichervolumen fassen kann. Durch welche Variable dieser Vorteil bewirkt werden könnte, wäre der Inhalt weiterführender Studien. Zu vermuten wäre eine Strategie, anhand welcher 
mithilfe des Wissen um phonematische Abfolgen in der Sprache die wahrgenommene und verarbeitete Teilinformation nach Wahrscheinlichkeitsregeln ergänzt würde.

Alle Aufgaben zur phonologischen Bewusstheit wurden von lese-/rechtschreibschwachen Kindern schlechter als von Kontrollkindern bewältigt, so dass die empirische Vorhersage 1.a bestätigt wurde.. Demzufolge kann von einer eingeschränkten Fähigkeit zur Operation sowohl mit einzelnen Phonemen als auch mit ganzen Silben oder Wortteilen ausgegangen werden.

Das dritte Konstrukt der Triade phonologischer Verarbeitungsfähigkeiten, der Abruf aus dem Langzeitgedächtnis, erwies sich ebenfalls als bei lese-/rechtschreibschwachen Probanden im Vergleich zu gesunden Probanden beeinträchtigt und führte damit zur Bestätigung der empirischen Vorhersage (1.1).

Da damit alle Tests auch gemäß der empirischen Vorhersage 1.0 ausgefallen waren, blieb kein Zweifel an deren Bestätigung bzw. an der Bewährung der psychologischen Hypothese 1.0, die deutliche Schwächen der phonologischen Verarbeitung lese-/rechtschreibschwacher Personen postulierte.

Durch die Bestätigung der pH 1.0 war eine Säule des Versuchsaufbaus realisiert worden.

\subsubsection{EIN WEITERFÜHRENDER ASPEKT}

Nachdem alle untersuchten Teilkomponenten des Arbeitsgedächtnisses sich als Lese-/Rechtschreibschwächen-spezifisch leistungsschwächer herausgestellt hatten, keimt die Frage nach der Funktionsfähigkeit deren Regulationseinheit, der zentralen Exekutive, auf. Möglicherweise ist deren Effizienz bei Lese-/rechtschreibschwachen ebenfalls eingeschränkt. Diese Überlegung wird durch zwei Befunde genährt, die in diese Richtung weisen: Unter den Variablen befanden sich die rückwärts zu repetierende Zahlenspanne und das Farbstriche Benennen. Beide sind auch zur Erfassung exekutiver Funktionen geeignet und wiesen gemäß der Faktorenanalyse in Punkt 5.3.1.2. Gemeinsamkeiten auf, die in der Tat im Sinne exekutiver Funktionen interpretiert werden können. Diese Variablen hatten -wie alle phonologischen Variablen- signifikante Gruppenunterschiede zu Ungunsten der lese-/rechtschreibschwachen Gruppe erbracht. 


\subsection{DISKUSSION DER BEFUNDE ZUR AUDITIV SENSORI- SCHEN VERARBEITUNG}

\subsubsection{PSYCHOLOGISCHE HYPOTHESE}

Im neurophysiologischen Teil der Untersuchung war es in allen sechs Aufgabenbedingungen gelungen, eine Mismatch Negativity hervorzurufen. In jeder Bedingung wurden zwei Parameter (Fläche; Peaklatenz) erhoben; die meisten Mismatch Negativity - Kurven ließen sich weiterhin in Segmente unterteilen. Drei der insgesamt 24 Variablen zeigten signifikante Gruppenunterschiede zu Ungunsten der lese-/rechtschreibschwachen Gruppe. Da mit der Anzahl von drei erwartungskonformen Variablen das Kriterium zufällig positiver Testausgänge von $5 \%$ überschritten wurde, wurde mit diesem Ergebnis die empirische Hypothese 2.0 bestätigt. Die Existenz von Verarbeitungsschwächen im auditiv sensorischen Gedächtnis lese/rechtschreibschwacher Probanden, wie sie von der psychologischen Hypothese 2.0 postuliert wurde, wird damit ebenfalls angenommen.

Diese erfassten Schwierigkeiten konnten nun mit den phonologischen Schwierigkeiten in Verbindung gebracht werden. Weiterhin erlaubte das Muster der Parameter, die Schwierigkeiten bereitet hatten, den Versuch, Rückschlüsse auf die Natur des Verarbeitungsdefizits im auditiven sensorischen Gedächtnis zu ziehen.

Die Variablen, die signifikante und hypothesenkonforme Gruppenunterschiede produziert hatten, stammten aus jeweils anderen MMN-Bedingungen: In der phonologischen Bedingung führte die Peaklatenz des Deviants /ga/ (zweites Segment) zu bedeutsamen Gruppenunterschieden, bei der nichtphonologischen Bedingung handelte es sich um den Flächenwert des dynamischen Deviants (zweites Segment), und in der Sinusbedingung erzeugte die Fläche des dynamischen Deviants (gesamte Kurve) die erwarteten Gruppenunterschiede.

Neben dem Befund, dass drei hypothesenkonforme Effekte von 24 möglichen Effekten eine relativ geringe Anzahl erwartungkonformer Testausgänge darstellt, ist auch zu erwähnen, dass die hypothesenkonformen Effekte nur geringe Effektstärken aufwiesen.

Auftretenszeitpunkt und Form der Potenziale werden ebenso wie die relative Schwäche des Effektmusters unter Einbezug von Befunden aus der Literatur in den folgenden Abschnitten diskutiert. In diesem Zusammenhang werden auch die Themenbereiche der internen sowie der Variablen-Validität angeschnitten. 


\subsubsection{EINBETTUNG DER BEFUNDE IN DIE LITERATUR}

\subsubsection{DESKRIPTIVE ASPEKTE DER PotenZIALE}

\subsection{ZEITFENSTER}

Gemäß Näätänen (1995) tritt die Mismatch Negativity gewöhnlich in einem Zeitfenster von 100 bis 300 ms nach Reizbeginn auf. In dieser Untersuchung erstreckte sich der als Mismatch Negativity interpretierbare Part der Differenzkurve (ebenso wie bspw. bei Schulte-Körne et al., 1998c) auf einen größeren Zeitraum (100-750 ms). Da die Verläufe von Stimuli und Kontrastbedingungen abhängig sind, mag in den komplexen Versuchsbedingungen der Grund für die höhere zeitliche Inanspruchnahme der Verarbeitung von Standard und Deviant zu suchen sein. Dies könnte wiederum durch eine Involvierung von Langzeitrepräsentationen zu begründen sein, einem Argument, das für die vorliegende Studie mit Sicherheit zutrifft und von Näätänen und Kollegen (1993b) selbst herangezogen wurde, als MMN-Potenziale dieser Arbeitsgruppe sich bis zu einem Zeitpunkt von etwa $500 \mathrm{~ms}$ nach Stimulus-Onset erstreckten.

Hier ist anbei zu bemerken, dass in keinem von 100 bis 300 ms liegenden Segment signifikante Gruppenunterschiede der MMN zu verzeichnen waren; diese lagen in „Teil B“, welcher etwa bei 300 ms einsetzte (PH-G: 300-740 ms; NP-D: 340-480 ms) bzw. betrafen die Gesamtfläche der MMN-Kurve (SIN-D: 100-680 ms). Auch dieser Befund ist bei Schulte-Körne et al. (1998c) zu finden. Die Beeinträchtigung lese-/rechtschreibschwacher Probanden in der auditiven Verarbeitung scheint demzufolge erst in den etwas späteren Verarbeitungsstadien deutlich zu werden. Dies ist konsistent mit der Annahme der Lese-/Rechtschreibschwäche als Verarbeitungs- und nicht als Perzeptionsstörung: Nach einer offenbar anfangs ungestörten Perzeption treten Schwierigkeiten bei deren Verarbeitung auf.

Da die Aufgabe des Spurenbildungsprozesses -welchen die MMN reflektiert- , darin besteht, unterschiedliche Informationen zu bündeln, läge es nahe, Schwierigkeiten beim Bündelungsprozess zu vermuten. Diese können dadurch bedingt sein, dass eine bestimmte Reizqualität nicht adäquat eingebunden werden kann. Ebenso ist ein dysfunktionales Instrument der Bündelung vorstellbar - diese Idee brachten Kujala et al. (2000) auf, indem sie ein verlängertes Integrationsfenster bei lese-/rechtschreibschwachen Personen annahmen. Überlegungen dieser Art nachzugehen kann eine Aufgabe zukünftiger Forschung sein. 


\subsection{FORM DER POTENZIALE}

Bei der Begutachtung der Form der Potenziale aus den phonologischen Bedingungen erstaunt zunächst, dass die hier erhaltenen Kurven nicht mit denen identisch sind, die Schulte-Körne et al. (1998c) mit sehr ähnlichen Bedingungen erhalten hatten. Deren MMN-Antwort war deutlich dreigipfelig, wohingegen die Potenziale der vorliegenden Studie ein bis zwei Gipfel aufwiesen. Allerdings lassen sich durchaus Erklärungen anführen: Auch wenn der Kontrast der Arbeitsgruppe wie hier sprachlicher Natur war; in den Details unterschied sich deren Bedingung von der hier angewandten. Es handelte sich um ein anderes Stimuluspaar (/da/, /ba/), eine andere Stimuluskonstellation (/ba/ als Deviant), eine andere Stimulusdauer (90 ms bei einem ISI von $590 \mathrm{~ms}$ ) und nicht zuletzt eine andere Produktion der Stimuli (synthetisch).

Das in dieser Studie gewonnene Potenzial aus der statischen Sinus-Bedingung entsprach weitgehend dem in der Veröffentlichung von Schulte-Körne et al. (1998c) aufgeführten, das die Gruppe durch die Kontrastierung desselben Reizpaares wie es hier angewandt wurde, gewonnen hatte.

Wichtiger als der Formvergleich ist möglicherweise anzumerken, dass das frühe MismatchPotenzial bei Schulte-Körne et al. (1998c) als MMN definiert wurde. Ein Gruppenunterschied der Potenzialdifferenz im selben Zeitraum wurde hingegen in dieser Studie nicht als MMN gewertet, da das MMN-Potenzial bzw. die zentrale Ladung auf dem Skalp positiv gepolt war.

\subsubsection{DISKUSSION DES SCHWACHEN EFFEKTMUSTERS}

\subsection{VERGLEICH MIT EFFEKTSTÄRKEN AUS DER LITERATUR}

Die drei signifikante Gruppenunterschiede produzierenden EEG-Variablen zeichneten sich durch geringe Effektstärken aus $\left(\eta^{2}: .068\right.$ bis .086; t-Wert: 1.8 bis 2.1). Ein Blick in die Literatur verrät vor allem, dass die Effektstärken dem Leser meistens vorenthalten werden. Berichtete Werte übersteigen allerdings deutlich die Größenordnung der hier erzielten Effektstärken (bspw. Schulte-Körne et al., 1998c: $\mathrm{p}=.0043$ / F=9.5 (Varianzanalyse) für die MMN-Fläche des phonologischen Kontrastes; Baldeweg et al., 1999: $p=.001$ (Varianzanalyse) für die MMNFläche bei Frequenzunterschieden). Diese lassen sich also schwer in einen Literaturkontext einordnen; was bleibt, ist die Aussage, dass stärkere Effekte möglich sind. Diese führt zur Frage, weshalb das Mögliche hier nicht gelungen ist. 
Damit verbunden ist die Frage, aus welchem Grund nicht beide Variablen einer Bedingung, die eine andere Modalität (Fläche bzw. Latenz) erfassten, signifikante Gruppenunterschiede aufwiesen. Beide Modalitäten waren als geeignet für die Aufzeichnung von Verarbeitungskapazitäten (siehe Punkt 2.4.3.) ausgewiesen worden. Bei den vorliegenden geringen Effektstärken liegt es nahe, zu vermuten, dieselben Tendenzen wären in den Parallelvariablen auch vorhanden, wären jedoch nicht statistisch signifikant geworden. Eine solche Vermutung ist jedoch $\mathrm{zu}$ verneinen: Die entsprechenden t-Werte betrugen 0 bis .3, die entsprechenden $\eta^{2}$ Werte rangierten zwischen .761 und .893. Da in der Literatur die Flächenvariable die gängigere ist und in vielerlei Paradigmen eingesetzt wurde, wo sich an ihr Gruppenunterschiede lese/rechtschreibschwacher und gesunder Probanden manifestierten, ist weiterhin eine Zuschreibung des Signifikant-Werdens auf systematische Zusammenhänge zwischen Bedingung und Messmodalität auszuschließen.

Wenn wie hier Gruppenunterschiede unter ihren möglichen Größenordnungen von Effektstärken bleiben bzw. mögliche Effekte nicht aufgetreten sind, ist die interne Validität der Untersuchung, in diesem Fall des neurophysiologischen Teils der Untersuchung und die Probandenselektion, in Frage zu stellen:

\subsection{INTERNE VALIDITÄT}

Da die Mismatch Negativity ein auditives Paradigma ist, gilt der erste kritische Gedanke dem Lärmpegel im Ableitungsraum. Die Versuchspersonen wurden von den Laborgeräuschen abgeschirmt, indem die Ableitung in einem isolierten Nebenzimmer stattfand und die Testsequenzen außerdem per Kopfhörer übermittelt wurden. Vermutlich war es dennoch unvermeidbar, dass zuweilen Geräusche an das Ohr der Versuchspersonen drangen. Die hohe Anzahl an Messzeitpunkten und die Artefaktbereinigung des EEGs nach der Ableitung sollten (u.a.) weiterhin den störenden Einfluss der einzelnen Nebengeräusche minimieren, die die praktischen Lärmisolierungsmaßnahmen nicht abhalten konnten.

Die Ableitungen selbst wurden stets von derselben entsprechend ausgebildeten und routinierten Person durchgeführt.

Weder Mängel der Ableitung noch störender Lärm sind daher als Ursache eventueller Ergebnisverzerrungen plausibel.

Weiterhin ist vorstellbar, dass das im Vergleich zum Diagnosekriterium des ICD 10 mildere Selektionskriterium für Lese-/Rechtschreibschwäche, das hier angelegt wurde (40 anstelle von 
37 als kritischem T-Wert im Rechtschreibtest), die Gruppenunterschiede zur Kontrollgruppe verringert hat und somit Ursache für falsche Hypothesenentscheidungen wurde.

Um den Einfluss einer eventuell zu milden Selektion zu prüfen, wurden die entsprechenden tTests nach Ausschluss der Personen wiederholt, die von der Kriteriumsdivergenz betroffen waren. Es handelte sich um drei Kinder. Es resultierten weder effektstärkere Gruppenunterschiede, noch in zwei Modalitäten sichtbare Gruppenunterschiede einer Bedingung. Der potentielle Vorwurf einer allzu milden Vergabe der Diagnose Lese-/Rechtschreibschwäche scheint infolgedessen ebenfalls nicht der Grund für die nur mäßigen Effekte im EEG-Bereich zu sein.

Ein Mangel an interner Vailidität scheint demzufolge das relativ schwache Effektmuster nicht erklären zu können.

\subsection{VARIABLENVALIDITÄT: DISKRIMINATIVE AUFGABENSCHWIERIGKEIT}

„... lese-/rechtschreibschwache Personen haben insbesondere Schwierigkeiten bei der Verarbeitung kleiner, nicht großer, Reizunterschiede... , fassen Kujala und Näätänen (2001; S. 538) in ihrem Review zusammen. Dieser Hinweis führt zu einer weiteren möglichen Ursache des relativ schwachen Effektmusters der neurophysiologischen Variablen in dieser Studie. Zwar war die Konzeption einiger Stimuli bzw. der Stimuluspaare an Befunden bereits bewährter Kontraste orientiert; dennoch mussten die Reize erst hergestellt werden, was bereits per se zu einem Anstieg der Ergebnisvariabilität führt. Zudem wurden in keinem Fall gegebene Versuchsanordnungen vollständig reproduziert, da innerhalb der eigenen Versuchsanlage Konsistenz gewahrt werden musste. Andere Stimuli entstanden gemäß theoretischer Maßgaben und waren somit völlig neue Untersuchungsgegenstände. Für angelehnte und besonders für neue Stimuluspaare gilt, dass der Forscher mit ihnen relativ blind in die Fähigkeitenlandschaft greift. Um Gruppenunterschiede zu erhalten, muss eine Aufgabenschwierigkeit appliziert werden, die von Gesunden noch, von Lese-/rechtschreibschwachen nicht mehr (einwandfrei) bewältigt wird. Die Zone der diskriminativen Aufgabenschwierigkeit ist wahrscheinlich sehr eng. Es ist folglich möglich, dass die Paradigmata der Bedingungen, in welchen Gruppenunterschiede auftraten, die vorhandenen Verarbeitungsschwächen nicht optimal identifizieren konnten. (Mit der Pilotstudie zur Angleichung der Entdeckungswahrscheinlichkeiten der verschiedenen Stimuli hatte diese Problematik eventuell eingedämmt, jedoch nicht ausgeschaltet werden können.)

Denkbar ist insofern, dass manche ebenbürtige Leistung der Experimentalgruppen einem Deckeneffekt zuzuschreiben ist. (Da in jeder Bedingung eine Mismatch Negativity abgeleitet 
werden konnte, sind Bodeneffekte unwahrscheinlicher.) Für eine solche Vermutung käme insbesondere die Bedingung PH-P infrage, da sie keine Gruppenunterschiede aufdeckte, obwohl die andere phonologische Bedingung es tat: Die Differenz der Voice-Onset-Time zwischen /b/ und /p/ ist größer als zwischen /b/ und /g/ und kompensiert damit möglicherweise die Diskriminationsschwierigkeiten, die durch den gleichen Verlauf der Formanten entstehen. Im mitteldeutschen Sprachraum wird zudem akkurat zwischen harten und weichen Konsonanten unterschieden, so dass dieses Paradigma auch für lese-/rechtschreibschwache Kinder bereits zu geübt sein könnte.

Unsicherheit bezüglich des Vorliegens von Decken- oder Bodeneffekten führt letztlich zum Infragestellen aller negativen Resultate einer Untersuchung, die jedoch ebenso bedeutend wie die positiven Ergebnisse sind. Lediglich valide Parameter mit bekannten Schwierigkeitsgraden könnten derartige Zweifel ausräumen. Es wäre aus diesem Grund die Anstrengung wert, in systematischer Feinarbeit derartige valide Parameter bereitzustellen. Hierbei müssten für jeweils einen konkreten Parameter unterschiedliche Schwierigkeitsgrade entwickelt und einer Normierung ähnlich getestet werden. Da die Diskrimination unterschiedlicher Reizqualitäten von der Muttersprache abhängt, würde diese Aufgabe jeder Nation für sich obliegen.

Diese kritischen Überlegungen zur Validität der neurophysiologischen Parameter stellen die Bewährung der psychologischen Hypothese bzw. der empirischen Vorhersage 1.0 nicht in Frage, da die Aufdeckungskraft existierender Gruppenunterschiede, nicht die Echtheit detektierter Gruppenunterschiede angezweifelt wurde, welche zur Bewährung der Hypothese geführt hatten.

\subsubsection{DISKUSSION DER SPEZIFIKATION DES AUDITIV SENSORISCHEN VERARBEITUNGSDEFIZITS LESE-/RECHTSCHREIBSCHWACHER PROBANDEN}

\subsubsection{EMPIRISCHE VORHERSAGEN}

Über die Natur eines auditiv sensorischen Verarbeitungsdefizits standen drei konkurriernde Hypothesen im Raum, die die Basis für drei psychologische Hypothesen bildeten ( $\mathrm{pH}$ 2.g, pH 2.s, $\mathrm{pH}$ 2.t). Die neurophysiologische Untersuchung war so gestaltet worden, dass mithilfe des resultierenden Musters Gruppenunterschiede erzeugender Variablen das (mögliche) Verarbei- 
tungsdefizit Probanden zu spezifizieren sein würde. Dieser Sachverhalt drückte sich in drei empirischen Vorhersagen aus (eV 2.g, eV 2.s, eV 2.t).

Das Ergebnismuster sprach für eine Annahme der empirischen Vorhersage 2.t.

In dieser war von einem auditorischen Defizit die Rede, welches auf Schwierigkeiten der Verarbeitung rasch aufeinanderfolgender Reize beruht. Die beiden anderen empirischen Vorhersagen zur Theorie eines generellen, die gesamte auditive Verarbeitung gleichmäßig umfassenden Defizits bzw. zur Theorie eines rein sprachlichen Defizits, wurden abgelehnt. Vor der Entscheidung über die Bewährung / Nicht-Bewährung der psychologischen Hypothesen bedürfen jedoch sowohl die beiden Annahme- als auch die Ablehnungsentscheidungen über die empirischen Vorhersagen der Diskussion:

\subsection{ABLEHNUNG EINES GENERELLEN VERARBEITUNGSDEFIZITS}

Die negativen Ergebnisse hinsichtlich Gruppenunterschieden bei der Diskrimination von Frequenzunterschieden sind stimmig mit den Befunden anderer Autoren wie beispielsweise Mody (1993) oder Schulte-Körne et al. (1998c, 1999b). In diese Reihe lässt sich sogar allerdings äußerst vorsichtig- eine Untersuchung von Csépe und Gyurkócza (1995) stellen, in welcher lese-/rechtschreibschwache Kinder in ihrer MMN-Amplitude hinter Kontrollprobanden deutlich zurücklagen, wenn ein Frequenzkontrast von 1000 versus $1050 \mathrm{~Hz}$ appliziert wurde. Da weder die Latenzvariable desselben Kontrastes noch die Amplitude oder Latenz eines Kontrastes von 1000 versus $1200 \mathrm{~Hz}$ Gruppenunterschiede erzeugte, in anderen Bedingungen der Studie Gruppenunterschiede jedoch deutlich konsistentere Auftretensmuster zeigten, werteten die Autoren das Ereignis selbst nicht.

Die Abwesenheit positiver Ergebnisse bezüglich der Diskrimination der statischen Frequenzkontraste bzw. die Ablehnung der entsprechenden empirischen Vorhersage in dieser Studie kann dennoch nicht als unumstößliches Argument für die Schlussfolgerung begriffen werden, Unterschiede bei der Verarbeitung von Frequenzkontrasten oder im Sinne eines generellen Defizits seien mit Sicherheit auszuschließen. Baldeweg et al. (1999) gestanden ihren Probanden nicht mehr als eine 50 millisekündige Stimulusdauer (bei einem großzügigen Interstimulusintervall von 1 sek) für die Diskrimination von Frequenzunterschieden $\mathrm{zu}$, die zwischen lediglich 15 bis $90 \mathrm{~Hz}$ rangierten. Dass bei Differenzen von 15, 30 und $60 \mathrm{~Hz}$ gruppenabhängige Leistungsunterschiede auftraten, nicht jedoch bei $90 \mathrm{~Hz}$, weist darauf hin, dass in der vorliegenden Studie möglicherweise zu leichte Bedingungen gewählt wurden (siehe oben). 
Auf der Basis dieser Überlegungen wird aus der Ablehnung der empirischen Vorhersage eV 2.g nicht die Nicht-Bewährung der psychologischen Hypothese pH 2.g abgeleitet: Das Vorliegen eines generellen auditiven Verarbeitungsdefizits konnte in der vorliegenden Arbeit demzufolge nicht ausgeschlossen werden.

\subsection{ABLEHNUNG EINES SPEZIFISCH SPRACHLICHEN VERARBEITUNGSDEFIZITS}

Das spezifisch sprachliche Defizit wird von Schulte-Körne et al. (1999b) postuliert, ebenso wie von Csépe und Gyurkócza (1995). Beide Gruppen hatten lese-/rechtschreibschwachen und gesunden Probanden verschiedene Reizbedingungen vorgegeben, wobei sich jeweils bei den phonologischen Kontrasten der einzige (Schulte-Körne et al., 1999b) bzw. die deutlichsten (Csépe und Gyurkócza, 1995) Leistungsunterschiede zwischen den Gruppen abzeichneten.Diese Position muss nicht unbedingt in hartem Widerspruch zu den Ergebnissen dieser Studie stehen. Die hiesige Ablehnung richtet sich gegen das spezifisch sprachliche Defizit als Primärursache der schriftsprachlichen Störung (d.h. das spezifisch sprachliche Defizit), nicht gegen sein Auftreten per se (d.h. nicht das sprachliche Defizit). Im Gegenteil: eine Vorstellung, gemäß welcher basale auditorische Defizite sich bis auf das Niveau der Schriftsprache hin auswirken, verlangt geradezu den Nachweis der Erschwernis einzelner Sprachfragmente. In diesem Sinne könnte der Leistungsunterschied in der Bedingung PH-G interpretiert werden.

Die Vereinbarkeit der Ergebnisse betrifft insbesondere die Studie von Csépe und Gyurkócza (1995), da hier keine Untersuchungen zur temporalen Verarbeitung vorgenommen wurden. Dennoch ficht diese Studie die vorliegende an. Sie tut dies insofern, als in allen phonologischen Kontrastbedingungen - welche dieselben waren wie in der vorliegenden Arbeit - signifikante Gruppenunterschiede beobachtet werden konnten. Die Ableitungsbedingungen, sowie die Anzahl und die Selektion der Versuchspersonen waren mit den hiesigen nahezu identisch. In einem einzigen Punkt jedoch unterschieden sich die Vorgehensweisen: Der Parallelisierung. Csépe und Gyurkócza (1995) matchten die Gruppen anhand eines Handlungs-IQs (KTR: 113; LRS: 114), der eine Standardabweichung über dem dieser Arbeit lag (KTR: 98; LRS: 96), so dass die Gruppe insgesamt bereits ein höheres Intelligenzniveau aufwies. Damit verbunden ist das Niveau und die zwischen den Gruppen zugelassene Diskrepanz des Verbal-IQs. Beide Punkte sollten in der vorliegenden Studie möglichst nah am Durchschnitt gehalten werden. So betrug der Verbal-IQ der lese-/rechtschreibschwachen Gruppe in etwa (102) so viel wie deren 
parallelisierter Handlungs-IQ. Der Verbal-IQ der Kontrollgruppe lag mit nur zehn Punkten mehr keine ganze Standardabweichung darüber. Der Verbal-IQ der lese-/rechtschreibschwachen Gruppe bei Csépe und Gyurkócza (1995) betrug 90, der der Kontrollgruppe 120. Damit blieb die lese-/rechtschreibschwache Gruppe mit ihrer Verbalintelligenz beinahe zwei Standardabweichungen hinter ihrer Handlungsintelligenz und mehr als zwei Standardabweichungen hinter der Verbalintelligenz ihrer gesunden Altersgenossen zurück. Beides mag sich im Sinne einer Präselektion für verbale und die damit verbundenen auditiv sensorischen Fähigkeiten ausgewirkt haben, so dass für das Auftreten von Gruppenunterschieden sehr förderliche Bedingungen geschaffen wurden.

Aufgrunddessen wurde auch in diesem Punkt keine unbedingte Widersprüchlichkeit zu den Befunden der vorliegenden Arbeit gesehen.

Weiterhin bilden Nachweise von Leistungsunterschieden zwischen lese-/rechtschreibschwachen und Kontrollgruppen bei nonsprachlichen Paradigmata wie Unterschiede von Tonabfolgen (Schulte-Körne et al., 1999a), Frequenzunterschieden (Baldeweg et al., 1999) oder eines Rhythmusses (Kujala et al., 2000) Argumente gegen die These, ein Verarbeitungsdefizit Lese-/rechtschreibschwacher setze erst auf der sprachlichen Ebene ein.

In diesem Punkt erschienen die Ergebnisse der vorliegenden Arbeit mit Berichten aus der Literatur konsistent. Aus diesem Grund wurde die Ablehnung der empirischen Vorhersage eV 2.s in die Entscheidung zur Nicht-Bewährung der psychologischen Hypothese $\mathrm{pH}$ 2.s umgesetzt.

\subsection{ANNAHME EINES DEFIZITS DER RASCH TEMPORALEN VERARBEITUNG}

Aus einem einzigen negativen Befund kann niemals die Abwesenheit einer ganzen Kategorie möglicher Auffälligkeiten geschlossen werden. Das jedoch tun Schulte-Körne et al. (1999b), wenn sie aus dem Nicht-Auftreten von Gruppenunterschieden bei einer Pausenentdeckungsaufgabe ableiten, im auditiven sensorischen Gedächtnis spielten rasch temporale Verarbeitungsschwierigkeiten allenfalls eine untergeordnete Rolle. Zudem handelte es sich bei der entsprechenden Aufgabe um ein aktives und damit um ein für das sensorische Gedächtnis nicht optimales Paradigma. Da die Marburger Arbeitsgruppe (1999a; siehe oben) zudem in einer Veröffentlichung desselben Jahres von ihrer klaren Favorisierung eines spezifisch sprachlichen Defizits Abstand nehmen, um einem Defizit der temporalen Verarbeitung den Vorrang 
einzuräumen, werden die Befunde nicht als Argument gegen eine Annahme eines Defizits der rasch temporalen Verarbeitung gewertet.

Der Vollständigkeit halber seien hier nochmals die ebenfalls unterstützenden Leistungsunterschiede bei der Diskrimination von Rhythmusunterschieden von Kujala et al. (2000) aufgeführt. Insgesamt gehören Schwierigkeiten der temporalen Verarbeitung zu den in der Literatur am besten belegten. Da darauf aufbauend auch Gruppenunterschiede bei der Verarbeitung zeitlicher Gegebenheiten innerhalb von Konsonanten nachgewiesen wurden (Chiappe et al., 2001; Leppänen et al., 2002) wundert hier, dass der Kontrast /ba/ versus / pa/ keine gruppenabhängigen Leistungsunterschiede aufdecken konnte. Wie bereits oben diskutiert, bestünde die Möglichkeit, dies einer zu einfachen Gestaltung des Kontrastes zuzuschreiben: Chiappe et al. (2001) arbeiteten mit Kontinua; Leppänen et al. (2002) wählten in ihrer Sprache noch relativ ungeübte Säuglinge.

Die Hypothese eines Defizits der rasch temporalen Verarbeitung stellt sich folglich auch unter Berücksichtigung der Literatur in einem recht plausiblen Licht dar, so dass die psychologische Hypothese $\mathrm{pH}$ 2.t als bewährt gelten kann.

\subsubsection{WEITERFÜHRENDE ASPEKTE}

\subsection{SPEKTRALE KOMPLEXITÄT UND „SPRACHLICHKEIT“ DER SPRACHE}

Mody (1993) und Mody et al. (1995) hatten aufgrund ihrer Versuchsreihen die Ansicht vertreten, weder in der „Sprachlichkeit“ der Sprache, noch in einer der Sprache zugrundeliegenden spektralen Komplexität (siehe auch: Jaramillo et al., 1999) lägen besonders schwer zu bewältigende Anforderungen an die Verarbeitung lese-/rechtschreibschwacher Personen.

Die Überlegung hinsichtlich der Komplexität wurde explizit in die Konzeption der Stimuli einbezogen - und konnte bestätigt werden: Für eine untergeordnete Rolle der spektralen Komplexität spricht zum einen, dass der statische Frequenzkontrast keine gruppenabhängigen Verarbeitungsunterschiede aufzeigte, auch wenn er durch die komplexen sprachlichen Stimuli getragen wurde. In dieselbe Richtung weist das Auftreten von Gruppenunterschieden in beiden dynamischen Frequenzkontrastbedingungen, und damit unabhängig davon, ob der Frequenzkontrast durch unkomplexe Sinustöne oder durch die spektral komplexe Silbe /ba/ getragen wurde. 
Der negative Befund hinsichtlich des sprachlichen Kontrastes /ba/ versus /pa/ stützt die Ablehnung der reinen „Sprachlichkeit“ als alleinigem kritischen Punkt der lese-/rechtschreibschwachen Verarbeitung.

Aus ihren Untersuchungen zogen Mody (1993) und Mody et al. (1995) die Schlussfolgerung, das Verarbeitungsdefizit rasch aufeinanderfolgender Reize käme bei der Diskrimination eng benachbarter phonologischer Kategorien zum Tragen. In dieser Hinsicht kann der Arbeitsgruppe mit den Ergebnissen der vorliegenden Studie nicht zugestimmt werden: Die Deviants der dynamischen Bedingungen veränderten auf rasche Art und Weise lediglich das physikalische Merkmal „Frequenz“; es wurde kein Wechsel einer phonologischen Kategorie vorgenommen.

\subsubsection{2. ÜBERLEGUNGEN ZU EINEM VERLÄNGERTEN MMN-INTEGRATIONS-}

\section{FENSTER}

Als physiologische Grundlage für ein Verarbeitungsdefizit rasch temporaler Reize boten Kujala et al. (2000) die These eines verlängerten Integrationsfensters für die Fusion der einzelnen Spuren der verschiedenen Stimuluseigenschaften zu einer ganzheitlichen Repräsentation an. Diese These könnte ein überraschendes Phänomen erklären: Nach der Herstellung der dynamischen Deviants traten Zweifel an deren Effizienz auf, da sie einen charakteristischen „wellenförmigen“ Klang besaßen, der - dem Urteil erwachsener Hörer gemäß - die Diskrimination des Frequenzkontrastes eher zu erleichtern denn zu erschweren schien. Wirft man einen Blick auf die Flächenwerte der Kontrollgruppe, so findet sich dieser Verdacht bestätigt: Bei phonologischen Stimuli reagierten Kontrollkinder (im Segment B) auf die statische Frequenzänderung (NP-S) mit einer MMN-Fläche von -1.6, und auf die dynamische Frequenzänderung (NP-D) mit einer MMN-Fläche von $-2.6 \mu V x m s$; statische Sinus-Deviants (SIN-S; Segment B) wurden mit einer Fläche von -1.4, dynamische Sinus-Deviants (SIN-D) mit einer Fläche von -2.4 $\mu \mathrm{Vxms}$ quittiert. Bei lese-/rechtschreibschwachen Kindern verlief der Trend allerdings in die entgegengesetzte Richtung: Mit $-1.6 \mu \mathrm{Vxms}$ reagierten sie auf den phonologischen statischen Frequenzdeviant (NP-S), mit $-1.0 \mu \mathrm{Vxms}$ reagierten sie auf den dynamischen Deviant (NP-D). $-2.1 \mu \mathrm{Vxms}$ erreichte ihr diskriminatives Potenzial beim statischen Sinus-Deviant (SIN-S; Segment B) und fiel beim dynamischen Sinus-Deviant auf -.9 (SIN-D).In einer Varianzanalyse mit Messwiederholung, zeichnet sich ein signifikanter Interaktionseffekt zwischen den Faktoren „Gruppe“ und „Art des Frequenzkontrastes“ ab ( $\mathrm{F}=5.4 ; \mathrm{p}=.024)$. Ersetzt man hierbei korrekterweise das Segment B des statischen Sinusdeviants durch dessen Gesamtfläche, führt das zu keiner Änderung des Effekts $(\mathrm{F}=5.5 ; \mathrm{p}=.023)$. Man könnte also vermuten, die 
dynamische Bedingung war den Kontrollkindern in der Tat leichter, den lese-/rechtschreibschwachen Kindern jedoch schwerer gefallen. Ein verlängertes Integrationsfenster würde den Eindruck eines reduzierten und verschwommenen anstelle eines zurückgenommenen Kontrastes vermitteln und wäre damit konsistent mit den vorhandenen Resultaten.

Eine weitere Verknüpfung ist möglich: Eingangs wurde von einem Vorteil dysphasischer Probanden beim Nachsprechen verzerrter Kunstworte berichtet (Hasselhorn et al., 1995; Hasselhorn, 1999; vgl. Grube et al., 1998). Dieses Phänomen, das den Eindruck verleiht, als läge ein permanentes Rauschen über dem Hörerlebnis und das mit unpräziser Verarbeitung im phonetischen Speicher in Verbindung gebracht wurde, konnte in dieser Arbeit für Lese/rechtschreibschwache repliziert werden. Genügen die Verarbeitungsmechanismen rascher temporaler Reize nicht den Ansprüchen der schnellen Sprache - beispielsweise bedingt durch ein verlängertes Integrationsfenster-, ist ein verschwommener Höreindruck eine logische Konsequenz.

\subsubsection{ZUSAMMENFASSUNG DER DISKUSSION ZUR SPEZIFIKATION EINES AUDITIV SENSORISCHEN DEFIZITS}

Werden die Ergebnisse in den Zusammenhang der Literatur gestellt, büßt die anfängliche aus Annahme und Ablehnung der empirischen Vorhersagen resultierende Favorisierung des rasch temporalen Defizits ihren Absolutheitsanspruch ein. Insbesondere eine Studie von Baldeweg et al. (1999) veranlasste zur Vorsicht bei der Ablehnung von Verarbeitungsunterschieden in der Frequenzwahrnehmung bzw. bei der Ablehnung eines generellen auditiven Defizits. Die psychologische Hypothese 2.g hatte sich damit nicht bewährt. Das Vorliegen spezifisch sprachlicher Verarbeitungsdefizite konnte vehementer abgelehnt werden, da in dieser sowie in anderen Arbeiten nonsprachliche Bedingungen Leistungsschwächen lese-/rechtschreibschwacher Personen aufdeckten. Die psychologische Hypothese 2.s galt damit als nicht bewährt. Weitgehend in Einklang mit der Literatur befand sich die Annahme eines rasch temporalen Defizits, welche zur Bewährung der psychologischen Hypothese 2.t führte. Hier konnten zusätzliche Feststellungen getroffen werden: Der Befund, dass weder die „Sprachlichkeit“, noch die spektrale Komplexität die kritischen Punkte der lese-/rechtschreibschwachen Verarbeitung trafen, bestätigte Arbeiten der Gruppe um Mody (Mody, 1993; Mody et al., 1995). Allerdings wurde deren Vermutung, es sei die Diskrimination phonologischer Kategorien betroffen, nicht recht gegeben. Desweiteren konnte ein Interaktionseffekt zwischen dem 
Gruppenfaktors und der Art einer Frequenzänderung mit dem Postulat verlängerter Integrationsfenster (Kujala et al., 2000) in Verbindung gebracht werden. Ebenso war es möglich, die auch hier nachgewiesene verminderte Verarbeitungspräzision des phonetischen Speichers lese-/rechtschreibschwacher Probanden argumentativ auf eine Integrationsfensterverlängerung bzw. den Effekt einer inadäquaten Verarbeitung rasch temporaler Reize zurückzuführen. 


\subsection{ANALYSE DES ZUSAMMENHANGS PHONOLOGISCHER UND AUDITIV SENSORISCHER VERARBEITUNGSDEFIZI- TE BEI LESE-/RECHTSCHREIBSCHWACHEN KINDERN}

Die Diskussion der Zusammenhangsanalysen erfolgt wie der Ergebnisbericht in zwei Etappen: Zunächst (Punkt 6.3.1.) werden die Analysen rund um die psychologische Hypothese 3a thematisiert, in welcher es hauptsächlich um den Nachweis eines Zusammenhangs und weiterhin um die Spezifikation dieses Zusammenhangs ging (Punkt 6.3.1.3.). Im zweiten Teil werden die Ergebnisse zur Modellierung des Zusammenhangs diskutiert (Punkt 6.3.2.).

\subsubsection{DISKUSSION DER ANALYSEN ZUM VORLIEGEN EINES ZUSAMMEN- $\underline{\text { HANGS }}$}

\subsubsection{PSYCHOLOGISCHE HYPOTHESE 3A}

Konvergierende Befunde beeinträchtigter Leistungsfähigkeit lese-/rechtschreibschwacher Probanden sowohl bei kognitionspsychologischen als auch bei neurophysiologischen Untersuchungen führten zu der psychologischen Hypothese 3a, zwischen diesen bestehe ein Zusammenhang. Die Voraussetzung für die Prüfung dieser Hypothese bildeten die in dieser Arbeit erfolgreich replizierten Verarbeitungsschwierigkeiten lese-/rechtschreibschwacher Kinder im Vergleich zu gesunden Kindern. Die Ergebnisse der Analyse zeigten recht eindeutig, dass zwischen den auditiv sensorischen und phonologischen Verarbeitungsschwierigkeiten lese/rechtschreibschwacher Kinder Zusammenhänge bestehen, indem sie -im Sinne der empirischen Vorhersage 3a- gemeinsame Varianzanteile bei der Vorhersage der Rechtschreibleistung aufdeckten. Diese psychologische Hypothese wurde damit bestätigt. 


\subsubsection{VALIDITÄT DER ANALYSE}

Für die Analyse waren Berechnungen nötig, in welchen es bisweilen um sehr geringe Varianzbeträge ging. Das Hantieren mit allzu kleinen Werten könnte die Validität einer Analyse einschränken, da die Stichprobenabhängigkeit und die „Zufälligkeit“ von Ergebnissen steigen, je kleiner betreffende Werte sind bzw. je punktgenauer abgeleitete Aussagen formuliert werden. Allerdings wurde die Entscheidung, auch minimale Bereiche in die Berechnungen einzubeziehen, aus dem Ziel heraus getroffen, für die größeren Varianzbereiche, -die im Mittelpunkt des Interesses standen-, eine maximale interne Konsistenz und damit eine maximale Validität des Gesamtbildes zu gewährleisten.

Durch diese Einhaltung der maximalen Genauigkeit im System der vorliegenden Datenstruktur ist schließlich davon auszugehen, dass für die Beantwortung der Hypothese sowie für grobkörnigere weiterführende Deskriptionen bezüglich der Struktur der einzelnen Komponenten ein ausreichendes Maß an Validität erreicht war.

\subsubsection{ZUSAMMENHÄNGE ZWISCHEN KONSTRUKTEN UND EBENEN}

\subsection{ZUSAMMENHANG ZWISCHEN DEN KOGNITIONSPSYCHOLOGISCHEN KONSTRUKTEN UND DEN EEG-VARIABLEN}

Die Größenordnung der Überschneidung des EEG-Bereichs mit dem der kognitionspsychologischen Konstrukte betrug knappe 30\% (der Rechtschreibvarianz). Der Begriff „Überschneidung“ impliziert eigentlich die Existenz eigenständiger Anteile beider Komponenten, was für die EEG-Variablen nicht gegeben war: Mit Ausnahme nicht nennenswerter Anteile von jeweils ca. einem halben Prozent fiel die gesamte durch das EEG erklärte Varianz in einen Bereich, der von den kognitionspsychologischen Konstrukten bereits abgedeckt wurde und ca. $77 \%$ der Rechtschreibvarianz ausmachte. Die minimal additiven Varianzanteile gingen von den Variablen SIN und PHO aus.

Auf der Basis der genauen Strukturanalyse der Varianzanteile der kognitionspsychologischen Konstrukte konnte eine Aussage darüber getroffen werden, zu welchem Konstrukt die stärkste Affinität von seiten der EEG-Variablen bestand. Es handelte sich hierbei um das Arbeitsgedächtnis. Dieses beherbergte die gesamte Varianz der EEG-Variablen mit Ausnahme des 
bereits erwähnten außerhalb befindlichen einen Prozentes. Das Arbeitsgedächtnis war dabei das einzige Konstrukt der kognitionspsychologischen Reihe, welches den Hauptanteil der Variable PHO abdeckte.

Die Varianzen der beiden Variablen NP und SIN befanden sich nahezu vollständig auch im Bereich der phonologischen Bewusstheit. Somit ist dieses das an zweiter Stelle zu nennende Konstrukt hinsichtlich des Ausmaßes bestehender Gemeinsamkeiten zum EEG-Bereich. An letzter Stelle stand der Abruf aus dem Langzeitgedächtnis, da er lediglich mit Anteilen der EEG-Variablen Überschneidungsbereiche aufwies.

\subsection{ZUSAMMENHANG UNTER DEN KOGNITIONSPSYCHOLOGISCHEN KON- STRUKTEN}

Der aufdringlichste Befund der Strukturanalyse der kognitionspsychologischen Konstrukte besteht in der Mehrfachdetermination der die Rechtschreibleistung erklärenden Varianzanteile. Den Maximalwert von ca. 77\% füllten sowohl die phonologische Bewusstheit (ca. 70\%) als auch das Arbeitsgedächtnis (ca. 60\%) annähernd aus; dennoch waren die Bereiche der beiden Konstrukte etwas gegeneinander verschoben, so dass die Hinzunahme des jeweils anderen das Erklärungspotenzial dennoch signifikant erweitern konnte. Der Abruf aus dem Langzeitgedächtnis nahm einen vergleichsweise geringen Varianzraum von ca. 30\% ein; dieser wurde in den weitesten Teilen sowohl vom Arbeitsgedächtnis als auch von der phonologischen Bewusstheit erklärt.

\subsection{ZUSAMMENHANG UNTER DEN EEG-VARIABLEN}

Die EEG-Variablen wurden mehr durch die Erhebungsmethodik denn durch inhaltliche Kriterien geeint. Das Bild gemeinsamer Varianzen innerhalb des EEG-Bereichs war mit dieser Überlegung vollständig schlüssig: Die Variable PHO, deren Stimuli nur zum Teil, und deren Kontrast keinerlei Gemeinsamkeiten mit den Variablen NP und SIN hatte, wies in Folge keine gemeinsamen Varianzanteile mit den beiden anderen Variablen auf. Diese jedoch verlangten beide die Diskrimination eines dynamischen Frequenzkontrastes, so dass deren Varianzen wiederum über einen Überschneidungsbereich verfügten. 


\subsubsection{ANMERKUNGEN ZU EINZELNEN KONSTRUKTEN}

\subsection{PHONOLOGISCHES ARBEITSGEDÄCHTNIS}

Mit Varianzerklärungsanteilen an der Rechtschreibleistung von $62 \%$ von seiten des phonologischen Arbeitsgedächtnisses und von 71\% von seiten der phonologischen Bewusstheit kann davon ausgegangen werden, dass beide Konstrukte eine bedeutsame Rolle bei der Vorhersage der Rechtschreibstörung einnehmen. Damit unterstützen die Resultate der vorliegenden Studie die Position Hasselhorns et al. (2000b) sowie die Befunde der Längsschnittstudien von Klicpera \& Gasteiger-Klicpera (1995), Näslund \& Schneider (1996), Schneider \& Näslund (1993) oder Weinert \& Schneider (1987, 1992): Im Gegensatz zu Behauptungen aus englischsprachigen Ländern spiele das phonologische Arbeitsgedächtnis im deutschen Sprachraum für die Diagnose und Verursachung der Lese-/Rechtschreibstörung -neben dem Abruf aus dem Langzeitgedächtnis und der phonologischen Bewusstheit- eine bedeutsame Rolle. Es war vermutet worden, der Unterschied zwischen den Nationen würde durch die längere Wortlänge im Deutschen bedingt, welche das phonologische Arbeitsgedächtnis im Allgemeinen stärker belaste, oder wäre der schreibleistungsfokussierten Diagnose in deutschen Sprachraum zuzuschreiben.

Obschon offenbar das phonologische Arbeitsgedächtnis im deutschen Sprachraum für die Rechtschreibleistung und damit für die Diagnose und Verursachung der Lese-/Rechtschreibstörung unübersehbare Rolle spielt, wird dadurch die phonologische Bewusstheit jedoch nicht ihrer Bedeutsamkeit enthoben. Dass diese beiden Konstrukte geeignet sind, sich zu ergänzen, obwohl ihre jeweilige eigene Varianzstärke schon beträchtlich ist, zeigte die StepwiseRegression bei der Zusammenfassung der Konstrukte: Das Modul band zur Vorhersage der Rechtschreibleistung beide Konstrukte in die Variablenauslese ein.

Als Repräsentanten des Konstrukts Arbeitsgedächtnis wurden durch die Stepwise-Technik drei Variablen aggregiert. Diese waren die langsilbigen Items des Mottier-Tests, der Trigramm-Test und die Interaktionsvariable des Kunstwort-Nachsprechens. In dieser Auswahl manifestieren sich die drei untersuchten Funktionsmerkmale der phonologischen Schleife, nämlich das Speichervolumen durch die Mottier-Items, die Rehearsal-Geschwindigkeit durch den Trigramm-Test, und - dies ist besonders bemerkenswert - die postulierte Funktion „Speicherpräzision" (Grube et al., 1998; Hasselhorn, 1999) durch die in dieser Arbeit eingeführte Interaktionsvariable. Die Interaktionsvariable war als dritte Variable nach der Mottier- und der 
Trigramm-Variablen durch die mathematische Prozedur ausgewählt worden. Dabei wurde sie anderen Speichervolumenindikatoren (wie auch den Schleifenkapazitätsindikatoren) vorgezogen und damit als erklärungsrelevanter erachtet. In ihr zeigt sich offenbar ein Aspekt des phonologischen Arbeitsgedächtnisses, der durch die bekannten Variablen nicht erfasst wird. Das Postulat Hasselhorns und Kollegen wird demzufolge durch die Resultate unterstützt, ebenso wie die eingeführte Präzisionsvariable selbst. Dass diese Variable jedoch unter den repräsentativen Variablen die am schwächsten varianzerklärende war, kann als Impuls für deren Weiterentwicklung verstanden werden. Dabei ist jedoch einschränkend zu erwähnen, dass sie zu individualdiagnostischen Zwecken nicht einzusetzen sein wird, da sie sich aus einer doppelten Differenz herleitet, was die Interpretation interindividueller Unterschiede erschwert.

Ein anderer Aspekt der Auslese besteht darin, dass die Gedächtnisspannen beiseite gelassen wurden. Offenbar besaß das Agglomerat der Indikatoren der einzelnen Funktionsmerkmale eine höhere Aussagekraft für die Charakterisierung der phonologischen Schleife als die Spannenvariablen, die als Indikatoren der funktionellen Schleifenkapazität gelten.

\subsection{PHONOLOGISCHE BEWUSSTHEIT}

Die optimale Besetzung zur Repräsentation der phonologischen Bewusstheit alleine bestand in einer Kombination aus Variablen, die die phonologische Bewusstheit im engeren Sinne erfassen („Laute Ersetzen“, „Laute Vertauschen“), und aus einer Variablen, die die phonologische Bewusstheit im weiteren Sinne untersucht („Laute Klassifizieren). Das „Laute Klassifizieren“ erwies sich im Gegensatz zum „Laute Ersetzen“ und zum „Laute Vertauschen“ für die Vorhersage der Rechtschreibleistung als weniger bedeutsam, wenn neben den Variablen der phonologischen Bewusstheit auch Variablen des Arbeitsgedächtnisses in die Prädiktion eingebunden wurden. Offenbar besitzt die Fähigkeit der Manipulation von Phonemen eine größere Aussagekraft hinsichtlich der Vorhersage der Rechtschreibleistung als die Fähigkeit zur Manipulation von Morphemen. 


\subsection{ABRUF AUS DEM LANGZEITGEDÄCHTNIS}

Es wundert, dass der Varianzanteil des Abrufs aus dem Langzeitgedächtnis weniger als die Hälfte der beiden anderen kognitionspsychologischen Konstrukte betrug. Die wahrscheinlichste Erklärung dafür besteht vermutlich weniger in einem geringeren realen Erklärungspotenzial des Konstrukts, als vielmehr darin, dass dieses Konstrukt durch lediglich eine Variable vertreten wurde. Daraus erwächst die Aufforderung an eine folgende Untersuchung, mehr Variablen zur Erfassung dieses Konstrukts zur Verfügung zu stellen. Für diese Studie bedeutet die obige Überlegung eine eingeschränkte Validität hinsichtlich aller Aussagen bezüglich des Ausmaßes der Bedeutsamkeit des Abruf aus dem Langzeitgedächtnis bei der Erklärung der Rechtschreibleistung bzw. der Lese-/Rechtschreibschwäche. 


\subsubsection{DISKUSSION ZUR MODELLIERUNG DES ZUSAMMENHANGS}

\subsubsection{PSychologische HyPOTHESE 3B}

\subsection{BEWÄHRUNGSSTATUS}

Aufgrund konzeptueller Ähnlichkeiten zwischen Repräsentationen des auditiv sensorischen Gedächtnisses, wie sie mithilfe der MMN abgegriffen werden, und Spuren des phonetischen Speichers im Arbeitsgedächtnis, aufgrund der Überlegung, dass die Anforderung an die Komplexität der Verarbeitung bei der phonologischen Bewusstheit der Komplexitätsanforderung beim Rechtschreiben wahrscheinlich näher ist als die eines anderen Konstrukts, und schließlich aufgrund der konzeptuellen Ähnlichkeit zwischen der Abruffunktion aus dem Langzeitgedächtnis und dem Steuerungsmodul des Arbeitsgedächtnisses wurden Thesen über eine Struktur sukzessiver Einflussnahme der Konstrukte von der Ebene auditiv sensorischer Verarbeitung bis hin zur Rechtschreibleistung aufgestellt.

Die psychologische Hypothese $3 b$ hatte demzufolge ein Modell favorisiert, dessen Anfang durch die EEG-Variablen gebildet würde. Als folgende Etappe würde sich das Arbeitsgedächtnis anschließen, das wiederum von der phonologischen Bewusstheit gefolgt würde. Diese wäre der direkte Prädiktor der Rechtschreibleistung. Der Abruf aus dem Langzeitgedächtnis stünde in Beeinflussung durch das Arbeitsgedächtnis und würde selbst auf die phonologische Bewusstheit Einfluss nehmen. Für das EEG war ein weiterer direkter Einfluss auf die Rechtschreibleistung eingeräumt worden.

Allerdings ist es schwierig, die Ergebnisse der Hypothesenprüfung zu beurteilen: Die Beziehungen sowohl unter den kognitionspsychologischen Konstrukten als auch von den EEG-Variablen aus zu diesen waren vorhersagegemäß zu beobachten. Allein erlaubte das Modell, in welchem sich diese Beziehungen manifestierten, nicht seine Akzeptanz, da durch die vorgegebene Struktur zu wenig Varianz erklärt wurde, das heißt, die kritische Schwelle des Gütekriteriums GFI unterschritten wurde. Das postulierte Modell $\mathrm{zu}$ akzeptieren wäre damit falsch. Nichtsdestotrotz erwies sich keine andere Modell-Lösung als bessere sinnvolle Alternative; und in jeder Variante bestätigten sich die postulierten Beziehungen (weitestgehend) erneut. Daher wäre es ebenso nicht richtig zu behaupten, die Zusammenhänge zwischen den Konstrukten bzw. zwischen der neurophysiologischen Verarbeitungsebene, den kognitions-psychologischen 
Konstrukten und der Rechtschreibleistung seien völlig anders gestaltet, als es die Hypothese postulierte. Diese Situation entspricht einem Dilemma.

\subsection{EIN WEG AUS DEM DILEMMA}

Um einen Weg aus dem Dilemma zu finden, wurden folgende Überlegungen angestellt:

Die Goodness of Fit - Indizes kennzeichnen die Menge erklärter Varianz im Variablensystem. Da die Beziehungen zwischen (latenten) Variablen in der Realität nie so einfach sind, wie sie in einem theoretischen Modell formuliert und geprüft werden, erhöht das schlichte Hinzufügen von Variablen zu einem System nicht nur die Menge an Varianz, sondern ebenso die Menge an im Modell unerklärbarer Varianz. Das vorliegende Modell besitzt sieben latente Variablen, die sich aus zehn Messvariablen ableiten. Dies ist ein hoher Grad an Komplexität, vor allem wenn man einbezieht, dass die Messvariablen der kognitionspsychologischen Konstrukte auch stark interkorrelieren. Es erschien demzufolge als ein zu rechtfertigender Versuch, das System zu simplifizieren. Die inhaltlich am wenigsten bedeutungsschwerste, zudem durch einen relativ geringen Pfadkoeffizienten und nicht in direkter Linie von physiologischer Ebene zur Rechtschreibung verbundene Variable war der Abruf aus dem Langzeitgedächtnis. Im Gegensatz zu den anderen prädiktiven kognitionspsychologischen Konstrukten konstituierte sich dieses nur aus einer einzigen Messvariable, so dass deren Validität ebenfalls nicht mit der der beiden anderen kognitionspsychologischen Konstrukte vergleichbar war (siehe auch Punkt 6.3.1.4.3.). Auf diese Variable schien demzufolge am ehesten verzichtbar, wenn die Gesamtvarianz des Systems reduziert werden sollte, ohne dass für die Aussagekraft hinsichtlich der zu prüfenden Beziehungen größere Einbußen hingenommen werden mussten.

Es entstand folgende Modell-Lösung: 


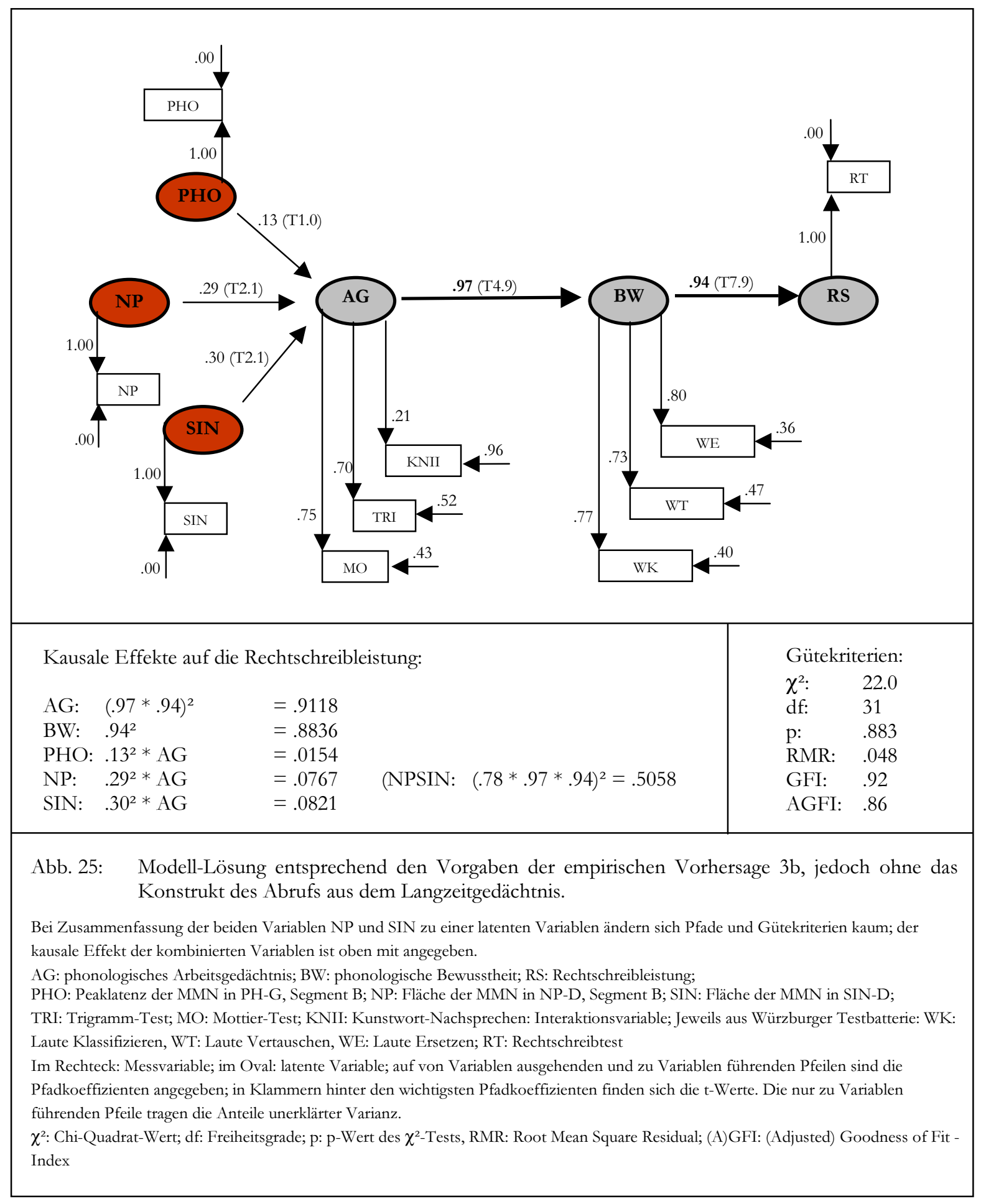

Die Gütekriterien weisen dieses Modell als annehmbar aus.

Hinsichtlich zweier Punkte ergaben sich Unterschiede im Vergleich zur Lösung des Modellpostulats: Zum einen entfiel der Einfluss der Variable PHO auf die Rechtschreibleistung. Dies 
wurde als keine dramatische Änderung begriffen, da dieser Pfad im postulierten Modell ohnehin lediglich eine Option darstellte und sich zudem als der schwächste aller EEG-Pfade erwiesen hatte. Auffällig war der kausale Effekt des Arbeitsgedächtnisses: Sein Gesamteffekt stieg auf die Größenordnung des Effekts der phonologischen Bewusstheit (.88) und übertraf diesen sogar leicht (.91). Auch dies konnte hingenommen werden, da das Arbeitsgedächtnis nichtsdestotrotz weiterhin in der Position vor der phonologischen Bewusstheit einzuordnen blieb.

Weitaus bedeutsamer als diese Unterschiede waren jedoch die Übereinstimmungen zwischen diesem und dem postulierten Modell bzw. dessen Variante mit direkten Pfaden aller kognitionspsychologischen Konstrukte zur Rechtschreibleistung. Die Befunde bezüglich der Elementfolgen und der Pfadgrößen reproduzierten sich beinahe vollständig - und manifestierten sich nun in einem Modell, welchem aufgrund seiner Gütekriterien (und der Abwesenheit unerklärbarer Artefakte) Annehmbarkeit attestiert würde, wäre es denn postuliert worden.

Mit der psychologischen Hypothese $3 b$ sollte dementsprechend wie folgt verfahren werden: Die Hypothese selbst konnte nicht als bewährt gelten: Diejenigen Fakten, die im Prüfmodell vorhanden waren, sich bereits in dessen erweitertem Alternativmodell bestätigt und zuletzt im Modell ohne den Abruf aus dem Langzeitgedächtnis repliziert hatten, sollen nichtsdestotrotz als höchstwahrscheinlich zutreffend erachtet werden. Es sind dies

* die starken Beziehungen zwischen den kognitionspsychologischen Konstrukten bzw. der kognitionspsychologischen Konstrukte auf die Rechtschreibleistung und damit verbunden die starken kausalen Effekte von phonologischer Bewusstheit und Arbeitsgedächtnis auf die Rechtschreibleistung,

* die zwar schwachen, aber vorhandenen Einflüsse (Pfade sowie kausale Effekte) der EEGEbene auf die Ebene der kognitionspsychologischen Verarbeitung,

* die Position der phonologischen Bewusstheit als direkten und die des Arbeitsgedächtnis als über die phonologische Bewusstheit gehenden indirekten Prädiktor der Rechtschreibleistung

* und die Nähe zwischen EEG-Variablen und Arbeitsgedächtnis.

* Der bemerkenswerte Effekt, dass das Zusammenfassen der Variablen NP und SIN zu einer enormen Erhöhung des kausalen Effekts führte, sollte ebenfalls festgehalten werden. 


\subsubsection{EINBETTUNG DER BEFUNDE IN LITERATUR}

\subsection{ZUR VORSTELLUNG VERZERRTER REPRÄSENTATIONEN AN DER BASIS DER STÖRUNG}

Eine der wichtigsten Aussagen der erzielten Befunde liegt darin, dass ein gerichteter Zusammenhang von der Ebene des auditiv sensorischen Gedächtnis über die Ebene der Kurzzeitgedächtnisprozesse hin zur Rechtschreibleistung gefunden wurde. Das ergänzt die Ergebnisse der multiplen Regression, die bereits von hohen gemeinsamen Varianzanteilen aller drei kognitionspsychologischen Konstrukte bei der Erklärung der Rechtschreibleistung zeugten, um den Kausalitätsgedanken. Immer wieder wird in der Literatur die Vorstellung von verzerrten Repräsentationen geäußert, die von perzeptiven Defiziten her rühren und als falsche bzw. zu unspezifische Muster im Langzeitspeicher die schriftsprachlichen Unsicherheiten der Lese-/Rechtschreibschwäche verursachen (z.B. Čeponiene et al., 1999; Chiappe, et al., 2001; Csépe \& Gyurkócza, 1995; Studdert-Kennedy \& Mody, 1995). Auch wenn durch die Ablehnung der Hypothese nicht von einer „Bewährung“ und aufgrund des Wegfalls des Abrufs aus dem Langzeitgedächtnis erst recht nicht von einer vollständigen Bewährung dieses Gedankens die Rede sein kann, können die extrahierten Befunde in dem Sinne deutlicher Hinweise auf die Richtigkeit dieser Überlegung gewertet werden. Mit Schulte-Körne et al. (1999b) ist die vorliegende Studie die einzige, die diese allgemein akzeptierte, aber bislang ungeprüfte Vorstellung zum Untersuchungsgegenstand machte.

Allerdings ist darauf hinzuweisen, dass das Vorhandensein kausal begründbarer Zusammenhänge lediglich die Aussage erlaubt, dass die aufeinanderfolgenden Prozesse in einer sinnhaften und gerichteten Beziehung zueinander stehen. Damit ist der Rückschluss gerechtfertigt, Resultate mangelhafter Verarbeitung im frühesten Schritt würden weitergegeben und sich letztendlich in der mangelhaften orthographischen Leistung manifestieren. Ob jedoch die schlechten Leistungen der lese-/rechtschreibschwachen Probanden bei Aufgaben des Arbeitsgedächtnis und der phonologischen Bewusstheit allein aus der Rezeption unpräzisen Materials zustande kommen, oder ob jeder weitere Prozess der Sprachverarbeitung für sich und zusätzlich beeinträchtigt ist, kann mit der gewählten Methode nicht geklärt werden. Hierbei wäre sogar für unterschiedliche Personen ein Beginn der defizitären Verarbeitung auf unterschiedlichen Etappen desselben Weges vorstellbar. Aufgrund der Heterogenität der Störung würden solche Befunde nicht überraschen. 


\subsection{VERGLEICH MIT DEM LISREL-MODELL VON SCHULTE-KÖRNE}

Schulte-Körne et al. (1999b) hatten, ebenso wie es in dieser Arbeit geschehen war, aus Variablen, die unterschiedliche Etappen der Sprachverarbeitung abbilden, ein LISREL-Modell berechnet. Es war ihnen gelungen, eine Verbindung von der Ebene des auditiven sensorischen Gedächtnis (einer MMN-Variable) bis hin zur Rechtschreibleistung zu ziehen. Zwischen den beiden Polen befand sich zuerst eine Variable zur aktiven Diskriminationsleistung und anschließend eine zur phonologischen Bewusstheit zählende Variable. Ein direkter Einfluss des EEGs auf die Rechtschreibung war ebenfalls Bestandteil des Modells.

In dem Nachweis der Verbindbarkeit unterschiedlicher Verarbeitungsebenen bzw. Konstrukten, von denen angenommen wird, dass sie zeitlich bzw. physiologisch aufeinander aufbauende Prozesse beinhalten, sowie in der Wahl der Pole, zwischen welchen der Zusammenhang geprüft wurde, entspricht das Modell der Marburger Arbeitsgruppe dem der vorliegenden Studie. Möglicherweise irritierend sind die guten Goodness of Fit - Indizes des SchulteKörneschen Modells (GFI $=.97, \mathrm{AGFI}=.88$ ), die im Gegensatz zu den hier erzielten Werten stehen. Der Unterschied ist jedoch mit der relativ geringen Varianz im Schulte-Körneschen Modell zu begründen, die wiederum darauf zurückgeht, dass sich dort jede latente Variable aus lediglich einer Messvariable konstituierte. (Ein vergleichshalber analog konzipiertes Modell erbrachte in diesem Datensatz eine perfekte Anpassung.) Trotz ungünstigerer Gütekriterien ist daher die vorliegende Analyse als nicht unvalider zu beurteilen.

Auf die Behauptung eines direkten Pfades von der physiologischen Ebene auf die Rechtschreibleistung kann hier nur vorsichtig Bezug genommen werden, da dieser Pfad im geprüften, aber nicht angenommenen Modellpostulat vorkam und im nicht-postulierten, aber annehmbaren Modell entfallen war. In Übereinstimmung mit Schulte-Körne et al. (1999b) war hierbei der Umstand, dass diejenige MMN-Variable, von der die direkte Wirkung ausging, durch die phonologische Kontrastbedingung erzeugt worden war. Da der Pfad jedoch schon im postulierten Modell sehr schwach (.14) war und sich durch das Wegfallen bei Modellveränderung offenbar als relativ labil erwiesen hatte, besteht hier die Tendenz, ihn eher für unbedeutend zu halten. Möglicherweise wird ein Teil der Varianz, die bei Schulte-Körne frei ist, in direkter Weise auf die Rechtschreibleistung gerichtet, während sie im Modell der vorliegenden Studie durch das Arbeitsgedächtnis weitgehend aufgefangen wird. Dies entspräche auch den Ergebnissen aus der Berechnung der multiplen Regression. 
Aus dem Befund, dass eine aktive Gap-Erkennungsaufgabe keine signifikanten Gruppenunterschied zwischen lese-/rechtschreibschwachen und gesunden Probanden ergeben hatte, hatten Schulte-Körne et al. (1999b), eine weitere wichtige Aussage ihrer Veröffentlichung abgeleitet (vgl. Punkt 6.2.3.1.3.). Sie hatten daraus geschlossen, Defizite in der rasch temporalen Verarbeitung würden für die Prädiktion der Schreibleistung eine untergeordnete Rolle spielen. Nicht nur die Befunde aus der Regressionsberechnung sprechen eine andere Sprache; auch die Befunde aus der Prüfung des hiesigen Modellpostulats stehen der Schulte-Körneschen Aussage entgegen: Die MMN-Variablen aus der nichtphonologischen und der Sinus-Bedingung, die beide durch eine zeitlich rasche Veränderung der Deviants (dynamische Deviants) charakterisiert waren, erzeugten auf das Arbeitsgedächtnis Pfade, die in ihrer Höhe denjenigen der phonologischen MMN-Variable sogar leicht übertrafen und zudem ein potentielles Signifikanzkriterium erfüllt hätten. Diese Befunde lassen eine Störungs-Verursachung durch Defizite der Verarbeitung rasch aufeinanderfolgender Reize eher wahrscheinlich denn unwahr-scheinlich erscheinen (siehe oben). Dieser Eindruck wird verstärkt durch den Effekt, dass die Zusammenfassung dieser beiden Variablen in jedem Modell eine enorme Steigerung des Pfadkoeffizenten bzw. des Kausaleffektes zur Folge hatte. Dies wirft nämlich die Vermutung auf, dass die nachweisbare Wirkung von seiten der elektrophysiologischen Ebene beträchtlich gesteigert werden könnte, wenn dem Paradigma nach weiterer künftiger Exploration mehrere bewährte Messvariablen zur Verfügung stünden, wie es bei den kognitionspsychologischen Konstrukten der Fall ist. Allerdings muss hier eingeräumt werden, dass dasselbe für den phonologischen Kontrast der Fall sein könnte, würde dieser durch mehrere verwandte Variablen repräsentiert.

\subsection{BEDEUTUNG VON ARBEITSGEDÄCHTNIS BZW. PHONOLOGISCHER BEWUSSTHEIT BEI DER VERURSACHUNG DER LESE-/RECHTSCHREIB- SCHWÄCHE}

Die Ansicht, das phonologische Arbeitsgedächtnis sei für die Verursachung und Diagnose der Lese-/Rechtschreibschwäche im deutschen Sprachraum nicht unbedeutender als die phonologische Bewusstheit, konnte bereits im vorangegangenen Abschnitt unterstützt werden (Hasselhorn et al., 2000b; Klicpera \& Gasteiger-Klicpera, 1995; Näslund \& Schneider, 1996; Schneider \& Näslund, 1993; Weinert \& Schneider, 1987, 1992): In keiner bislang veröffentlichten Arbeit wurden diese beiden Konstrukte in einer Konkurrenzsituation untersucht. Da dies hier der Fall war, konnte festgestellt werden, dass der Prädiktionsunterschied zwischen phonologischer Bewusstheit und Arbeitsgedächtnis weniger in dem Ausmaß der Bedeutung lag 
- dieses war in beiden Fällen stets hoch und wurde in Abhängigkeit von der Umgebung mitprädizierender Konstrukte mal für das eine, mal für das andere Konstrukt höher angegeben -, sondern in der Prädiktionssequenz. Die phonologische Bewusstheit als eine bereits sehr komplexe Fähigkeit erwies sich postulatsgemäß der schriftsprachlichen Leistung näher und war daher im Modell optimal als direkter Prädiktor unterzubringen. Die Aufgaben zum Arbeitsgedächtnis haben eher basalere, an zerebrale Konstitutionen anmutende Fähigkeiten zum Ziel. Auf der Grundlage dieser Fähigkeiten arbeiten nicht nur die für die Rechtschreibung, sondern auch die für die phonologische Bewusstheit notwendigen Prozesse. In der Konkurrenzsituation mit der phonologischen Bewusstheit ordnet sich daher das Arbeitsgedächtnis als indirekter Prädiktor der Rechtschreibleistung ein. 


\subsection{DISKUSSION DES GESAMTBILDES NACH ASPEKTEN}

\subsubsection{ZUSAMMENHANG ZWISCHEN DEN PRÄDIKTORISCHEN BEREICHEN}

Es war in der vorliegenden Studie gelungen, einen Zusammenhang zwischen zwei prädiktorischen Bereichen der Lese-/Rechtschreibschwäche nachzuweisen, nämlich zwischen der Informationsverarbeitung im auditiven sensorischen Gedächtnis, erfasst mit dem Mismatch Negativity - Paradigma, und der phonologischen Informationsverarbeitung.

Im Rahmen dieser Ermittlungen wurde deutlich, dass alle zur Vorhersage der orthographischen Leistung herangezogenen Konstrukte / Verarbeitungsebenen weitreichende gegenseitige Abhängigkeiten aufwiesen. Besonders starke Abhängigkeiten bildeten sich im Modell durch unmittelbare Sukzession der Konstrukte ab (EEG-Variablen und Arbeitsgedächtnis).

Auch wenn die Modellprüfung nicht zu einem bewährten Modell geführt hatte, wurde dennoch deutlich, dass die verschiedenen prädiktiven Bereiche bzw. Konstrukte als physiologisch aufeinander aufbauende Prozesse verstanden werden können. Damit gewinnt die Vermutung an Grundlage, unexakte Repräsentationen stünden am Beginn der Störung und würden sich über die verschiedenen Verarbeitungsstufen bis zur Ebene der schriftsprachlichen Leistung auswirken (z.B. Čeponiene et al., 1999; Chiappe, et al., 2001; Csépe \& Gyurkócza, 1995; Studdert-Kennedy \& Mody, 1995).

Die Abfolge der Ebenen / Konstrukte in dem Modell setzte mit der neurophysiologischen Ebene ein, an welche sich das Arbeitsgedächtnis anschloss. Das Bindeglied zwischen dem Arbeitsgedächtnis und der Rechtschreibleistung stellte die phonologische Bewusstheit dar.

Aufgrund einer ansonsten zu starken Multikollinearität der Daten wurden Aussagen bzgl. der Einflussnahme der Konstrukte / Verarbeitungsebenen nur aus einer Modellstruktur gezogen, in welcher der Abruf aus dem Langzeitgedächtnis nicht vertreten war. Das Fehlen des Abrufs aus dem Langzeitgedächtnis ist für die Vorstellung verzerrter Spuren am Beginn der Lese-/Rechtschreibschwäche jedoch besonders bedauernswert, da Langzeitrepräsentationen in einer solchen eine zentrale Rolle spielen.

An dieser Stelle sei der Zusammenhang der EEG-Ebene mit dem Arbeitsgedächtnis besonders hervorgehoben, da dieser den Knotenpunkt der Verknüpfung von neurophysiologischer mit kognitionspsychologischer Ebene bildete. Die Ausgangsbasis für die Suche nach einem Zusammenhang zwischen Verarbeitungsdefiziten beider Ebenen bildeten erstaunliche Parallelen zwischen Merkmalen des phonetischen Speichers und dem mit der Mismatch 
Negativity erfassten Spurenbildungsprozess des auditiven sensorischen Gedächtnis. In der Tat war der ermittelte Zusammenhang an dieser Stelle besonders eng. Grundlage dieser Aussage bildet der Befund, dass der Erklärungsbereich an Rechtschreibvarianz der EEG-Variablen sich so gut wie vollständig mit dem des Arbeitsgedächtnisses deckte, ebenso wie die Abfolge im LISREL-Modell, wo die EEG-Variablen ihre Hauptwirkung auf das Arbeitsgedächtnis ausübten. Čeponiene et al. (1999), die eine Verbindung zwischen dem Arbeitsgedächtnistest „Kunstwort-Nachsprechen“ und der Mismatch Negativity nachgewiesen hatten, werden hierin durch dieses Resultat unterstützt.

Die kognitionspsychologischen Konstrukte erreichten in der vorliegenden Studie ein Erklärungspotenzial von knapp 80\% der Rechtschreibvarianz, ein Maß, das bereits als hoch zu beurteilen ist. Ebenso wenig wie die EEG-Variablen ist hierbei der in dieser Studie erfasste Anteil der Variablen des Abrufs aus dem Langzeitgedächtnis notwendig. Wollte man folglich mit minimalem Aufwand und maximaler Effizienz die Rechtschreibleistung vorhersagen, so genügten laut dieser Arbeit die beiden Konstrukte Arbeitsgedächtnis und phonologische Bewusstheit, repräsentiert durch drei Arbeitsgedächtnis-Tests der Funktionsmerkmale „Rehearsal“, „Speichervolumen“ und „Speicherpräsision“, sowie durch die zur phonologischen Bewusstheit im engeren Sinne gehörigen Tests „Laute Ersetzen“ und „Laute Vertauschen“.

Der ca. 30\% große Varianzanteil, den die EEG-Variablen insgesamt zu erklären in der Lage waren, deckte sich (nahezu) vollständig mit den von den kognitionspsychologischen Konstrukten erklärten Anteilen. Dies war zwar im Sinne der Hypothese und implizierte, dass das Mismatch-Paradigma für die Rechtschreibprädiktion eine potentielle alternative Methodik zur Verfügung stellen könnte (vorausgesetzt, durch die Entwicklung weiterer MMN-Variablen würde der erklärte Varianzanteil der Rechtschreibleistung gesteigert). Ein Informationsgewinn, der über die Vorhersageleistung bisher angewandter, kognitionspsychologischer Methoden hinausgeht, wurde (hier) jedoch nicht erreicht.

Falls in einer weiterführenden Studie Interesse bestünde, mithilfe des MMN-Paradigmas additive Anteile zu finden, so würde laut der vorliegenden Arbeit sprachlichen Kontrasten die größte Entdeckungswahrscheinlichkeit eingeräumt, da die Variable PHO hier einen minimalen additiven Varianzbereich ausmachte bzw. teilweise einen direkten Einfluss auf die Rechtschreibleistung zeigte. 


\subsubsection{SPEZIFIKATION DES VERARBEITUNGSDEFIZITS BEI DER LESE-}

\section{/RECHTSCHREIBSCHWÄCHE}

Es wurde weiterhin untersucht, welcher Art ein frühes Verarbeitungsdefizit sein könnte, das als Mangel der Verarbeitungspräzision an unterschiedlichen Etappen der Sprachverarbeitung deutlich wird und welches möglicherweise von Verarbeitungsstufe zu Verarbeitungsstufe weitergereicht wird, bis es sich schließlich in einer Lese-/Rechtschreibschwäche manifestiert.

Die neurophysiologischen Messungen hatten nur wenige Gruppenunterschiede aufgedeckt. Diese wenigen genügten dennoch, um zum einen gruppenabhängige Verarbeitungsunterschiede im auditiven sensorischen Gedächtnis als existent $\mathrm{zu}$ erachten und mit Verarbeitungsunterschieden des Kurzzeitgedächtnis in Verbindung zu bringen und zum anderen, um Aussagen hinsichtlich einer Theorie zur Störungsverursachung im auditiven sensorischen Gedächtnis abzuleiten. Eine primäre Beeinträchtigung erst auf sprachlicher Ebene wurde deutlich abgelehnt, da diese Theorie nicht vereinbar mit den beiden Befunden von Verarbeitungsunterschieden bei nichtsprachlichen Bedingungen war. Diese beiden Variablen zeigten nicht nur gruppenabhängige Verarbeitungsunterschiede und waren in der Lage, ebenso wie die sprachliche MMN-Variable Anteile an der Rechtschreibvarianz zu erklären; die Varianzanteile, die durch die nonsprachlichen Variablen erklärt wurden, betrugen außerdem jeweils das Doppelte der Varianzerklärung der sprachlichen Variable. Dasselbe Bild bot die Modellierung: Alle drei MMN-Variablen zeigten Einflüsse auf das Arbeitsgedächtnis, wobei die Pfade der nichtsprachlichen Variablen sogar stärker als die der sprachlichen Variable waren.

Das hier erhaltene Muster von Gruppenunterschieden indizierenden EEG-Variablen deutete auf ein Defizit der rasch temporalen Verarbeitung hin. Allerdings konnte nach Einbezug der Literatur und dadurch aufkommender Kritik an der möglicherweise zu leichten Gestaltung der entsprechenden Kontraste ein generelles Defizit, die dritte geprüfte Theorie, ebenfalls nicht abgelehnt werden.

Die Theorie des temporalen Verarbeitungsdefizits ist ebenso wie die eines generellen Defizits mit einem Mangel an Verarbeitungspräzision in Einklang zu bringen. Diese Unterstützung von anderer Seite untermauert die These, dass bei Lese-/rechtschreibschwachen tatsächlich eines der beiden Verarbeitungsdefizite vorliegt. Da ein Defizit der temporalen Verarbeitung und ein generelles Defizit sich inhaltlich nicht ausschließen, ist es möglich, im Resümee beide Theorien nebeneinander stehen zu lassen. Somit konnte die Dreizahl der zur Verfügung stehenden Theorien nicht wie erhofft auf eine einzelne, aber immerhin auf zwei in Frage kommende Theorien eingegrenzt werden. 


\subsubsection{DAS KRITERIUM DER VERARBEITUNGSPRÄZISION}

Der Aspekt eines Mangels an Präzision der Informationsverarbeitung scheint gemäß der vorliegenden Datenlage für die Verursachung der Lese-/Rechtschreibschwäche eine Rolle zu spielen, da er in unterschiedlichen Kontexten bedeutsam wurde:

Auf kognitionspsychologischer Ebene konnte auf der Basis eines scheinbar paradoxen Effektes eine präzisionsbezogene Variable gebildet werden. Der Effekt bestand in einem Leistungsvorteil lese-/rechtschreibschwacher Kinder abbildete bei der Reproduktionsleistung verzerrter Items bei zweisilbigen Worten. Seine Paradoxie wurde durch eine Interpretation aufgelöst, gemäß welcher für lese-/rechtschreibschwache Kinder eine Gewöhnung an unpräzise Hörereignisse stattgefunden haben könnte und die ihnen bei kurzen und ihrem Speichervolumen angemessenen Items eine Art Trainingsvorteil verschaffen könnte.

Diese Variable wurde neben einem Geschwindigkeits- und einem Größenparameter als repräsentative Variable für das phonologische Arbeitsgedächtnis (von einer mathematischen Prozedur) ausgewählt, was die Relevanz der Variablen für das Arbeitsgedächtnis und sein Erklärungspotenzial der Rechtschreibleistung verdeutlicht.

Eine verminderte Verarbeitungspräzision ließe sich mit einer Vorstellung in Einklang bringen, die durch ein mit neurophysiologischen Methoden arbeitendes Team (Kujala et al., 2000) aufgebracht wurde. Es handelt sich um die Überlegung, ein Zeitfenster zur Integration einzelner Spuren zu einer Gesamtrepräsentation sei bei lese-/rechtschreibschwachen Personen verlängert und verwische auf diese Art und Weise den Höreindruck.

Eine derartige Integrationsfensterverlängerung böte für einen überraschenden Befund auf der neurophysiologischen Ebene, nämlich den des Interaktionseffekts der Gruppe mit der Art des Frequenzkontrastes, eine plausible Erklärung: Gesunden Probanden fiel die Diskriminierung des charakteristischen „wellenförmigen“ leichter als die des gleichmäßig erhöhten Deviants; lese-/rechtschreibschwachen Probanden erschien der „wellenförmige“ Deviant durch die verlängerte Integration möglicherweise weniger stark oder verwaschen erhöht, so dass ihnen die Diskrimination im Vergleich zum konstanten Frequenzkontrast schwerer fiel.

Somit konnte das Merkmal der Verarbeitungspräzison ebenenübergreifend nachgewiesen werden. 


\subsubsection{BEDEUTUNG DES ARBEITSGEDÄCHTNIS IM VERGLEICH ZUR PHONOLOGISCHEN BEWUSSTHEIT}

Wiederholt wurde die Debatte um die Bedeutung des Arbeitsgedächtnisses im Vergleich zur Bedeutung der phonologischen Bewusstheit für die Verursachung und Diagnose der Lese/Rechtschreibschwäche im deutschen Sprachraum aufgeworfen. Die Antwort, die auf der Basis der hier vorliegenden Resultate gegeben werden kann, vereinigt Forscher, die in dem einen Konstrukt, sowie Forscher, die dem anderen Konstrukt die Hauptrolle der Prädiktion zuschreiben.

Alle Tests beider Konstrukte führten zu effektstarken Gruppenunterschieden, beide erklärten beträchtliche Teile der Rechtschreibvarianz und beide zeigten im LISREL-Modell starke kausale Effekte auf die Rechtschreibleistung. Beide Konstrukte sind somit für die Prädiktion bedeutsam. Dies wird besonders in dem Befund deutlich, dass sich zur Erklärung der Rechtschreibvarianz eine Kombination aus beiden Konstrukten als die optimale VariablenZusammenstellung erwies.

Der Unterschied zwischen beiden Konstrukten bestand in der Nähe zur schriftsprachlichen Leistung: Die phonologische Bewusstheit als bereits komplexere Aufgabe nahm die Position als direkter Prädiktor der Rechtschreibleistung ein; das Arbeitsgedächtnis, das basalere Fähigkeiten prüft, übte seinen Einfluss auf die Rechtschreibleistung über die phonologische Bewusstheit und damit indirekt aus.

\subsubsection{METHODISCHE ÜBERLEGUNGEN}

$\mathrm{Zu}$ einer Positionierung des Abrufs aus dem Langzeitgedächtnis ließen sich keine Aussagen treffen, da diese Komponente lediglich Bestandteil nicht annehmbarer Modelliösungen war, was wiederum auf dessen relativ geringes Varianzerklärungspotenzial zurückzuführen war. Aus der relativ geringen Bedeutsamkeit des Abrufs aus dem Langzeitgedächtnis im vorliegenden Datensatz ließe sich möglicherweise auf die wahren kognitiven Gegebenheiten schließen. Dagegen steht jedoch der Einwand, dass das Element des Langzeitgedächtnisses einen wichtigen sinnhaften Knotenpunkt in einer Annahme zur Auswirkung verzerrter Repräsentationen auf die Rechtschreibleistung darstellen müsste. Wahrscheinlicher ist daher, dass durch die Repräsentation des Konstrukts durch nur eine Variable dessen Einfluss unterschätzt wurde. In 
einer Nachfolgestudie sollte von daher ein breiteres Spektrum entsprechender Variablen angeboten werden. Dabei wäre ebenso zu überlegen, ob das Konstrukt „Abruf phonologischer Codes aus dem Langzeitgedächtnis“ nicht um eine Langzeitspeicherkomponente phonologischer Codes erweitert werden könnte. Grund dieser Überlegung liegt ebenfalls in der relativ geringen ermittelten Bedeutsamkeit des Langzeitgedächtnis-Abrufs, da sie möglicherweise auch auf eine zu enge Fokussierung einer Fähigkeit hinweist.

Hinsichtlich der Bedeutsamkeit der Komponenten im Modell ist zu erwähnen, dass jeweils die von kognitionspsychologischen Konstrukten ausgehenden Pfade stets hoch waren, wohingegen die von EEG-Variablen ausgehenden Pfade schwache Koeffizienten trugen. Dies mag einer realen geringen Aufdeckbarkeit von Verbindungen zwischen neurophysiologischer und kognitionspsychologischer Ebene zuzuschreiben sein. Ebenso möglich sind jedoch wiederum methodische Ursachen: Eine größere Anzahl verwandter EEG-Variablen, die zu einer latenten Variable zusammenfassbar gewesen wären, hätten eventuell größere Varianzanteile erklären können; darauf weist der Befund hin, dass die Zusammenfassung zweier EEG-Variablen stets $\mathrm{zu}$ einer massiven Steigerung der Bedeutsamkeit geführt hatte. Die Exploration des MMNParadigmas und die damit verbundene Bereitstellung weiterer Variablen muss jedoch erst noch geleistet werden.

Auch in einem anderen Zusammenhang wurde die Exploration des MMN-Paradigmas vorgeschlagen: Um falsche Hypothesenentscheidungen vermeiden $\mathrm{zu}$ können, müssen Parameter zur Verfügung stehen, die einen optimalen gruppendiskriminierenden Schwierigkeitsgrad aufweisen. Um dies zu gewährleisten, müssten unterschiedlich schwierige Varianten des Zielparameters einer Art Normierung unterzogen werden.

\subsubsection{DIAGNOSTISCHE NUTZBARKEIT DES MMN-PARADIGMAS}

Wie die Definition gemeinsamer Anteile bei der Erklärung der Rechtschreibvarianz gezeigt hat, bieten die (hier eingesetzten) EEG-Variablen hinsichtlich der kognitionspsychologischen Tests eine alternative, keine additive, Erklärungsmöglichkeit. Aber auch diese Alternative bleibt im Ausmaß ihres Erklärungspotenzials weit hinter dem der kognitionspsychologischen Konstrukte zurück. Zum gegenwärtigen Entwicklungsstand ist das MMN-Paradigma folglich nicht als Ersatz der kognitionspsychologischen Tests bei der Diagnose einer Lese-/Rechtschreibschwäche vorstellbar. 
Es könnte jedoch hierfür eine Ausgangsbasis bieten:

Durch die Zusammenfassung der beiden frequenzkontrastierenden Variablen war deren kausaler Effekt auf die Rechtschreibleistung im LISREL-Modell drastisch angestiegen. Dies impliziert eine Steigerbarkeit des Erklärungspotenzials, wenn eine höhere Anzahl entsprechender MMN-Variablen zur Verfügung steht. Für die Ausgestaltung der MMN-Kontraste können keine einschränkenden Hinweise gegeben werden, da diejenigen hier angewandten Bedingungen, bei welchen es nicht zu Gruppenunterschieden kam, nicht als solche, sondern hinsichtlich ihres gruppen-(nicht)-diskrimierenden Schwierigkeitsgrades angezweifelt wurden. In diesem Sinne ist davon auszugehen, dass jede einzelne der hier applizierten (wie auch potentieller zukünftiger) Bedingungen in ihrer Erklärungskraft verbesserbar ist, wenn nach einer genauen Exploration der optimal gruppendiskriminierende Schwierigkeitsgrad dargeboten wird.

Die Entwicklung neuer MMN-Paradigmata mag auch zu Variablen führen, die hinsichtlich der kognitionspsychologischen Konstrukte additive Bereiche der Rechtschreibvarianz erklären.

Soll das MMN-Paradigma diagnostisch genutzt werden, müssten die oben genannten Verbesserungen erfolgen. Dies gilt insbesondere, wenn es als Frühdiagnostikum in die Routine einer kinderärztlichen Untersuchung Eingang finden soll. Es gälte hierbei, Bedingungen zu finden, die eine so starke Deutlichkeit und Reliabilität besitzen, dass die Ableitung innerhalb kurzer Zeit, das heißt anhand nur weniger Sweeps erfolgen kann. Wenn ein solches Paradigma gefunden ist, müsste es auf die gewünschte vorschulische Altersgruppe übertragbar sein und anhand dieser Gruppe genormt werden.

Es ist folglich festzuhalten, dass die bekannten und erprobten Mismatch Negativity Bedingungen durchaus eine Ausgangsbasis für einen künftigen physiologischen (Früh-) Indikator der Lese-/Rechtschreibschwäche bieten können; bis dieser jedoch zur Verfügung steht und routinemäßig einsetzbar ist, ist noch ein weiter Weg der Exploration, Konkretisierung, Verbesserung und Normierung zurückzulegen. 


\section{ZUSAMMENFASSUNG}

Im Mittelpunkt des Interesses standen in dieser Studie zwei unterschiedliche, mit der Verarbeitung von Sprache befasste Forschungstraditionen, da in deren beider Kontext Korrelate der Lese-/Rechtschreibschwäche berichtet werden: Aus der neurophysiologischen Forschung wurde zur Untersuchung der frühen Verarbeitungsstufe des auditiven sensorischen Gedächtnisses das Mismatch Negativity - Paradigma bekannt. Spätere Verarbeitungsprozesse wurden im Rahmen kognitionspsychologischer Ansätze definiert: es handelt sich um das phonologische Arbeitsgedächtnis, den Abruf aus dem Langzeitgedächtnis und die phonologische Bewusstheit. Zur Untersuchung dieser Konstrukte stehen bekannte paper-pencil-Tests bereit.

Das Ziel der vorliegenden Arbeit bestand darin, mögliche Gemeinsamkeiten zwischen den betreffenden Indikatoren aufzudecken und zu definieren. Daraus erhoffte man sich Implikationen für eine zukünftige Nutzbarkeit des MMN-Paradigmas als physiologisches Frühdiagnostikum der schriftsprachlichen Störung. Über eine gezielte Gestaltung des MMN-Paradigmas sollten außerdem Hinweise über die Art eines auf dieser Verarbeitungsstufe detektierbaren Verarbeitungsdefizits gewonnen werden.

In jedem untersuchten Bereich wurden in Einklang mit den Berichten der Literatur Verarbeitungsdefizite nachvollzogen. In der Tat zeigten sich weitreichende Überschneidungen der Varianzen, wenn man die Vorhersage der MMN-Variablen bzw. die Vorhersage der kognitionspsychologischen Konstrukte auf die Rechtschreibleistung verglich. Diese Überschneidungen waren derart gestaltet, dass das Prädiktionspotenzial des EEGs vollständig in dem der kognitionspsychologischen Konstrukte unterging. Somit konnten die MMN-Variablen eine Vorhersage der Rechtschreibleistung durch kognitionspsychologische Konstrukte eventuell teilweise ersetzen, jedoch nicht verbessern. Das bereits etwa 80\% der Rechtschreibvarianz umfassende Erklärungspotenzial von seiten der kognitionspsychologischen Konstrukte wurde vor allem durch die phonologische Bewusstheit mit ca. $70 \%$ und dem phonologischen Arbeitsgedächtnis mit ca. $60 \%$ getragen.

In besonderer Weise deckten sich die MMN-Varianzen mit denen des phonologischen Arbeitsgedächtnisses. So fügte sich auch die Arbeitsgedächtnis-Komponente in einem kausalanalytischen Modell an die des EEGs. Sie wurde von der phonologischen Bewusstheit gefolgt, welche schließlich die Rechtschreibleistung direkt vorhersagte. Die Tatsache, dass es möglich war, die verschiedenen Komponenten in einem Modell miteinander zu verknüpfen, zeigt die Existenz eines inhaltlichen Zusammenhangs der Prozesse auf. Dabei ist denkbar, dass eine 
Verarbeitungsschwäche in der frühesten Komponente an die darüberliegenden Prozesse weitergereicht wird und letztlich als Lese-/Rechtschreibschwäche sichtbar wird; es ist jedoch ebenso denkbar, dass die Funktion jeder einzelnen Etappe für sich (neu) gestört ist.

Das Muster der MMN-Variablen, welche gruppenabhängige Verarbeitungsunterschiede im auditiv sensorischen Gedächtnis aufgedeckt hatten, gestattete den Ausschluss eines spezifisch sprachliches Defizits. Ein generelles Verarbeitungsdefizit lag gemäß der Datenlage weiterhin im Rahmen des Möglichen. Am Plausibelsten erschien jedoch eine Störung der Verarbeitung rasch folgender Reize, da diese mit der Theorie eines verlängerten Integrationsfensters bei der Synthese der Spuren einzelner Stimuluseigenschaften zu einer Gesamt-Repräsentation erklärt werden kann, und diese Theorie wiederum auch andere (Neben-) Befunde der Studie zu integrieren in der Lage ist.

Diese Befunde „am Rande“ berühren die Thematik der Verarbeitungspräzision.

Unter anderem war hinsichtlich dieses Kriteriums aus dem Kunstwort-Nachsprechen eine Variable entwickelt worden, die im Folgenden nicht nur eine gruppenabhängige Differenz der Verarbeitungspräzision indizierte, sondern sich zudem neben den Merkmalen „Geschwindigkeit“ und „Speichervolumen“ als repräsentativ für das Arbeitsgedächtnis erwiesen hatte. Damit wurde die Variable an sich ebenso wie das Funktionsmerkmal der Verarbeitungspräzision bestätigt.

Obschon die neurophysiologische Messung Gruppenunterschiede aufzeigte, waren Anzahl und Stärke der vorhandenen Effekte gering. Es entstand der Verdacht, es seien weniger Gruppenunterschiede sichtbar geworden, als realiter vorlägen. Aus diesem Grunde wurden Überlegungen angestellt, das Mismatch - Paradigma zu optimieren: Hierbei gälte es zum einen, unterschiedliche Variablentypen zu entwickeln, wie zum anderen, bei diesen den jeweiligen optimalen gruppendiskriminierenden Schwierigkeitsgrad zu definieren. Diese Arbeitsschritte wären unbedingt notwendig, wenn das Paradigma eine (früh-)diagnostische Anwendung finden soll.

Aufgrund geringer Effektstärken bei den neurophysiologischen Untersuchungen ist bezüglich der Übertragbarkeit der entsprechenden Ergebnisse auf die Gesamtpopulation Vorsicht geboten. Die berichteten Ergebnisse und Schlussfolgerungen wurden mit dieser Vorsicht formuliert. 


\section{LITERATURVERZEICHNIS}

Aaltonen, O., Tuomainen, J., Laine, M. \& Niemi, P. (1993). Cortical differences in tonal versus vowel processing as revealed by an ERP component called mismatch negativity (MMN). Brain and Language, 44, 139-152.

Achenbach, T.M. (1991). Child Behavior Checklist 4-18. CBCL. Burlington, V.T.: University of Vermont, Department of Psychiatry.

Adams, M.J (1990). Beginning to read: Thinking and learning about print. Cambridge, MA: MIT Press.

Alho, K., Woods, D.L., Algazi, A. \& Näätänen, R. (1992). Intermodal selective attention. II. Effects of attentional load on processing of auditory and visual stimuli in central space. Electroencephaly and Clinical Neurophysiology, 82, 356.368.

Angermaier, M. (1974). Psycholinguistischer Entwicklungstest. Weinheim: Beltz.

Backhaus, K., Erichson, B., Plinke, W. \& Weiber, R. (1994). Multivariate Analysemethoden. eine anwendungsorientierte Einführung. 7. Aufl. Heidelberg: Springer.

Baddeley, A.D. (1982). Reading and working memory. Bulletin of the British Psychological Society, 35, 414-417.

Baddeley, A.D. (1986). Working memory. Oxford: Oxford University Press.

Baddeley, A.D. (1990). Human memory: Theory and practice. Hillsdale, NJ: Erlbaum.

Baddeley, A.D. (1994). The magical number seven: Still magic after all these years? Psychological Review, 101, 353-356.

Baddeley, A.D. (1996a). Exploring the central executive. Quarterly Journal of Experimental Psychology, 49A, 5-28.

Baddeley, A.D. (1996b). The concept of working memory. In: S.E. Gathercole (Hrsg.): Models of short-term memory (S.1-27). Hove: Psychology Press.

Baddeley, A.D., Gathercole, S.E. \& Papagno, C. (1998). The phonological loop as a language learning device. Psychological Review, 105: 158-173.

Baddeley, A.D. \& Hitch, G.J. (1974). Working memory. In: G. Bower (Hrsg.): The psychology of learning and motivation. Vol. 8, 47-90. San Diego, CA: Academic Press.

Baddeley, A.D. \& Salamé, P. (1986). The unattended speech effecht: Perception or memory? Journal of Experimental Psychology, 12, 525-29.

Baddeley, A.D., Thompson, N. \& Buchanan, M. (1975). Word length and the structure of short-term memory. Journal of Verbal Learning and Verbal Beahavior, 14, 575-589. 
Baddeley, A.D. \& Wilson, B. (1985). Phonological coding and short-term memory in patients without speech. Journal of Memory and Language, 24, 490-502.

Bakker, D.J. (1992). Neuropsychological classification and treatment of dyslexia. Journal of Learning Disabilities, 25 (2), 102-109.

Bakker, D.J. (1979). Perceptual asymmetries and reading proficiency. In: Bortner, M. (Hrsg.): Cognitive growth and development, S. 134-152. New York: Brunner / Mazel.

Bakker, D.J. (1986). Electrophysiological validation of L- and P-type dyslexia. Journal of Clinical and Experimental Neuropsychology, 8, 133.

Bakker, D.J. (1973). Ear dominance and reading ability. Cortex, 9, 301-312.

Baldeweg, T., Richardson, A., Watkins, S., Foale, C. \& Gruzelier, J. (1999). Impaired auditory frequency discrimination in dyslexia detected with mismatch evoked potentials. Annals of Neurology, 45, 495-503.

Banaschewski, T., Tiffin-Richards, M., Hasselhorn, M. \& Rothenberger, A. (2000). Komorbidität von hyperkinetischer Störung und Legasthenie am Beispiel phonologischer, semantischer und syntaktischer Sprachfähigkeiten bei Kindern. Sprache, Stimme, Gehör, 24, 106-112.

Bäumler, G. (1985). Farbe-Wort-Interferenztest (FWIT). Göttingen: Hogrefe.

Berkhan, O. (1885). Über die Schriftsprache bei Halbidioten und ihre Ähnlichkeit mit dem Stammeln. Archiv für Psychiatrie, 16, 78-86.

Berkhan, O. (1886). Über die Schriftsprache bei Halbidioten und ihre Ähnlichkeit mit dem Sprachgebrechen. 2. Stammeln und Stottern. Archiv für Psychiatrie, 17, 861-897.

Birkel, P. (1994a). Weingartener Grundwortschatz Rechtschreib-Test für erste und zweite Klassen (WRT 1+). Göttingen. Hogrefe.

Birkel, P. (1994b). Weingartener Grundwortschatz Rechtschreib-Test für zweite und dritte Klassen (WRT 2+). Göttingen. Hogrefe.

Birkel, P. (1994c). Weingartener Grundwortschatz Rechtschreib-Test für dritte und vierte Klassen (WRT 3+). Göttingen. Hogrefe.

Bishop, D.V.M., Carlyon, R.P., Deeks, J.M. \& Bishop, S.J. (1999). Auditory processing impairment: Neither necessary nor sufficient for causing language impairment in children. Journal of Speech, Lanaguage, and Hearing Research, 42, 1295-1310.

Blaesser, B. (1994). Die Bedeutung der phonologischen Bewusstheit für das frühe Lesen und schreiben: Theoretische Fundierung und Förderungsmöglichkeiten. Unveröffentlichte Dissertation, Universität Würzburg.

Bloom, A., Wagner, M., Reskin, L. \& Bergman, A. (1980). A comparison of intellectually delayed and primary reading disabled children on measures of intelligence and achievement. Journal of Clinical Psychology, 36, 788-790. 
Booth, J.R., Perfetti, C.A., MacWhinney, B. \& Hunt, S.B. (2000). The association of rapid temporal perception with orthographic and phonological processing in children and adults with reading impairment. Scientific Studies of Reading, 4, 101-132.

Bradley, L. \& Bryant, P.E. (1978). Difficulties in auditory organization as a possible cause of reading backwardness. Nature, 271, 746-747.

Bradley, L. \& Bryant, P.E. (1985). Rhyme and reason in reading and spelling. Ann Arbor: University of Michigan Press.

Brady, SA. \& Shankweiler, D.P. (Hrsg.) (1991). Phonological processes in literacy. Hillsdale, NJ: Erlbaum.

Broadbent, D.E. (1958). Perception and communication. New York: Pergamon Press.

Broadbent, D.E. (1984). The maltese cross: A new simplistic model for memory. Behavioral and Brain Sciences, 7: 55-94.

Bruck, M. (1990). Word recognition skills of adults with childhood diagnoses of dyslexia. Developmental Psychology, 26, 439-454.

Bryant, P., Bradley, L., MacLean, M. \& Crossland, J. (1989). Nursery rhymes, phonological skills and reading. Journal of Child Language, 16, 407-428.

Castles, A. \& Coltheart, M. (1993). Varieties of developmental dyslexia. Cognition, 47, 19-180.

Čeponiene, R., Service, E., Kurjenluoma, S., Cheour, M. \& Näätänen, R. (1999). Children's performance on pseudoword repetition depends on auditory trace quality: evidence from event-related potentials. Developmental Psychology, 35, 3, 709-720.

Cheour, M., Čeponiene, R., Lehtokoski, A., Luuk, A., Allik, J., Alho, K. \& Näätänen, R. (1998). Development of language-specific phoneme representations in the infant brain. Nature of Neuroscience, 1, 351-353.

Chiappe, P., Chiappe, D.L. \& Siegel, L. (2001). Speech perception, lexicality, and reading skill. Journal of Experimental Child Psychology, 80, 58-74.

Conrad, R. (1964). Acoustic confusion in immediate memory. British Journal of Psychology, $55,75-84$.

Cowan, N. (1984). On short and long auditory stores. Psychological Bulletin, 96: 341-370.

Cowan, N. (1988). Evolving conceptions of memory storage, selective attention, and their mutual constraints within the human information-processing system. Psychological Bulletin, 104: 63-191.

Cowan, N. (1995). Attention and memory. An integrated framework. Oxford: University Press.

Cowan, N., Winkler, I., Teder, W. \& Näätänen, R. (1993). Memory prerequisites of the mismatch negativity in the auditory event-related potential (ERP). Journal of Experimental Psychology: Learning, Memory, and Cognition, 19. 909-921. 
Csépe, V. \& Gyurkócza, E.E. (1995). Normal and disturbed phoneme perception: mismatch negativity in dyslexia. Handout of a COST A8 Workshop on phonology and reading disorders: A multidisciplinary approach held in Amsterdam.

Das, J.P., Mensink, D. \& Mishra, R.K. (1990). Cognitive processes separating good and poor readers when IQ is covaried. Learning and Individual Differences, 2, 423-436.

DeFries, J.C., Fulker, D.W. \& LaBuda, M.C. (1987). Evidence for a genetic etiology in reading disability for twins. Nature, 329, 537-539.

DeFries, J.C., Stevenson, J., Gillis, J.J. \& Wadsworh, S.J. (1991). Genetic etiology of spelling deficits in the colorado and London twin studies of reading disability. Reading and Writing Interdisciplinary Journal, 3, 271-283.

De Jong, P.F. (1998). Working memory deficits of reading disabled children. Journal of Experimental Child Psychology, 70, 75-96.

De Weirdt, W. (1988). Speech perception and frequency discrimination in good and poor readers. Applied Psycholinguistics, 9, 163-183.

Döpfner, M. \& Lehmkuhl, G. (1998). Diagnostik-System für psychische Störungen im Kindesund Jugendalter nach ICD 10 und DSM IV. Bern: Huber.

Duara, R., Kushch, A., Gross-Glenn, K., Barker, W.W., Jallad, B., Pascal, S., Loewenstein, D.A., Sheldon, J., Rabin, M., Lewin, B. \& Lubs, H. (1991). Neuroanatomic differences between dyslexic and normal readers on magnetic resonance imaging scans. Archives of Neurology, 48, 410-416.

Escera, C., Alho, K., Winkler, I. \& Näätänen, R. (1998). Neural mechanisms of involuntary attention to acoustic novelty and change. Journal of Cognitive Neuroscience, 10, 590-604.

Esser, G. (1991). Was wird aus Kindern mit Teilleistungsschwächen? Stuttgart: Enke.

Esser, G. \& Schmidt, M. (1994). Children with specific reading retardation - early determinants and long-term outcome. Acta Paedopsychiatrica 56, 226-237.

Evans, L.D. (1990). A conceptual overview of the regression discrepancy model or evaluating severe discrepancy between IQ and achievement scores. Journal of Learning Disabilities, 23, 406-412.

Farmer, M.E. \& Klein, R.M. (1995). The evidence for a temporal processing deficit linked to dyslexia: a review. Psychonomic Bulletin and Review, 2, 460-493.

Foorman, B.R., Francis, D.J., Fletcher, J.M. \& Lynn, A. (1996). Relation of phonological and orthographic processing to early reading: Comparing two approaches to regression-based, reading-level-match designs. Journal of Educational Psychology, 88, 639-652.

Ford, J.M., \& Hillyard, S.A. (1981). Event-related potentials (ERPs) to interruptions of a steady rhythm. Psychophysiology, 18, 322-330. 
Fowler, A.E. (1991). How early phonological development might set the stage for phoneme awareness. In: S. Brady \& D. Shankweiler (Hrsg.): Phonological processes in literacy: $A$ tribute to Isabelle Y. Liberman (S. 91-117). Hillsdale, NJ: Erlbaum.

Froster, U., Schulte-Körne, G., Hebebrand H. \& Remschmidt, H. (1993). Cosegregation of balanced translocation with retarded speech development and dyslexia. Lancet, 342, 178179.

Galaburda, A.M. (1988). The pathogenesis of childhood dyslexia. In: F. Plum (Hrsg.): Language, Communication, and the Brain. New York: Raven Press.

Galaburda, A.M. (1991). The reading brain: the biological basis of dyslexia. In: Duane, D.D. \& Gray, D.B. (Hrsg.): Parkton: York Press.

Galaburda, A.M., Aboitiz, F., Rosen, G.D. \& Sherman, G.F. (1986). Histological asymmetry in the primary visual cortex of the rat: Implications for mechanisms of cerebral asymmetry. Cortex, 22, 151-160.

Galaburda, A.M. \& Kemper, T.L. (1979). Cytoarchitectonic abnormalities in developmental dyslexia; a case study. Annals of Neurology, 6, 94-100.

Galaburda, A.M., LeMay, M., Kemper, T.L. \& Geschwind, N. (1978). Right-left asymmetries in the brain. Science, 199, 852-856.

Galaburda, A.M. \& Livingstone, M. (1993). Evidence for a magnocellular defect in developmental dyslexia. In: Tallal, P., Galaburda, A.M., Llinás, R.R. \& von Euler, C. (Hrsg.): Temporal information processing in the nervous system: special reference to dyslexia and dysphasia. Annals of the New York Academy of Sciences, New York, 682, 7082.

Galaburda, A.M., Sherman, G.F., Rosen, G.D., Aboitiz, F. \& Geschwind, N. (1985). Developmental dyslexia: Four consecutive cases with cortical anomalies. Annals of Neurology, 18, 222-233.

Gathercole, S.E. (1997). Models of verbal short-term memory. in: M.A. Conway (Hrsg.): Cognitive models of memory (S. 13-45). Hove: Psychology Press.

Gathercole, S.E. \& Baddeley, A.D. (1990). Phonological memory deficits in language disordered children: Is there a causal connection? Journal of Memory and Language, 29. 336-360.

Gathercole, S.E. \& Baddeley, A.D. (1993). Working memory and language. Hove: Erlbaum.

Gathercole, S.E., Willis, C., Baddeley, A.D. \& Emslie, H. (1994). The children's test of nonword repetition: a test of phonological working memory. Memory, 2, 103-127.

Gathercole, S.E., Willis, C.S., Emslie, H. \& Baddeley, A.D. (1991). The influences of syllabes and wordlikeness on children's repetition of nonwords. Applied Psycholinguistics, 12, 349367. 
Gathercole, S.E., Willis, C.S., Emslie, H. \& Baddeley, A.D. (1992). Phonological memory and vocabulary development during the early school years: A longitudinal study. Developmental Psychology, 28, 887-898.

Geschwind, N. \& Levitsky, W. (1968). Human brain: Left-right asymmetries in temporal speech region. Science, 161, 186-187.

Giard, M.H., Lavikainen, J., Reinikainen, K., Bertrand, O, Pernier, J. \& Näätänen, R. (1995). Separate representation of stimulus frequency, instensity, and duration in auditory sensory memory: An event-related potential and dipole-model study. Journal of Cognitive Neuroscience, 7, 133-143.

Godfrey, J.J., Syrdal-Lasky, A.K., Millay, K. \& Knox, C.M. (1981). Performance of dyslexic children on speech perception tests. Journal of Experimental Child Psychology, 32, 401-424.

Gomes, H., Bernstein, R., Ritter, W., Vaughan, H.G.,Jr. \& Miller, J. (1997). Storage of feature conjunctions in transient auditory memory. Psychophysiology, 34, 712-716.

Golkenrath (1984). Verständnis und Wandel des Legasthenie-Begriffes von den Anfängen bis zur Gegenwart. Dissertation. Technische Universität Aachen. Herzogenrath: Verlag Murken-Altrogge.

Goswami, U. \& Bryant, P. (1990). Phonological skills and learning to read. London: Erlbaum.

Gough, P., Ehri, L. \& Treiman, R. (1992). Reading acquisition. Hillsdale, NJ: Erlbaum.

Goyette, C.H., Conners, C.K. \& Ulrich, R.F. (1978). Normative data on revived Conners Parent and Teacher Rating Scales. Journal of Abnormal Child Psychology, 6, 221-136.

Graham, S.A. \& Kline, R.B. (1991). IQ differences among poor readers: do they matter? Unpublished manuscript, Concordia University, Montreal, Quebec.

Gratton, G., Coles, M.G. \& Donchin, E. (1983). A new method for off-line removal of ocular artifact. Electroencephalography and Clinical Neurophysiology, 55, 468-484.

Grimm, T., Noethen, M. \& Schulte-Körne, G. (1998). Zur Genetik der Legasthenie. Sprache, Stimme, Gehör 22, 8-12.

Grube, D. (1999). Arbeitsgedächtnis und Zeitverarbeitung im Alter. In: D.H. Rost (Hrsg.): Pädagogische Psychologie und Entwicklungspsychologie. Berlin: Waxmann.

Grube, D., Hasselhorn, M. \& Weiß, J. (1998). Altersdefizite im phonologischen Arbeitsgedächtnis: Spielt die Verarbeitungspräzision des phonetischen Speichers eine Rolle? Zeitschrift für Gerontopsychologie und-psychiatrie, 11, 3 -11.

Grund, M., Haug, G. \& Naumann, C. (1994). Diagnostischer Rechtschreibtest für 4. Klassen (DRT 4). Weinheim: Beltz.

Grund, M., Haug, G. \& Naumann, C. (1995). Diagnostischer Rechtschreibtest für 5. Klassen (DRT 5). Weinheim: Beltz.

Haslam, R.H., Dalby, J.T. \& Rademaker, A.W. (1981). Cerebral asymmetry in developmental dyslexia. Archives of Neurology, 38, 679-682. 
Hasselhorn, M. (1986). Differentielle Bedingungsanalyse verbaler Gedächtnisleistungen bei Schulkindern. Frankfurt/Main: Peter Lang.

Hasselhorn, M. (1992). Entwicklung des kategorialen Organisierens: Anfänge der Wissensnutzung als Strategie kindlicher Gedächtnisfähigkeiten. Unveröffentlichte Habilitationsschrift. Institut für Psychologie. Göttingen.

Hasselhorn, M. (1999). Wird der kindliche Dysgrammatismus durch Gedächtnisdefekte verursacht? In: DGS-Landesgruppe Sachsen e.V. (Hrsg.): Sprachheilpädagogik über alle Grenzen - Sprachentwicklung in Bewegung (S.102-106).

Hasselhorn, M., Grube, D. \& Mähler, C. (2000c). Theoretisches Rahmenmodell für ein Diagnostikum zur differentiellen Funktionsanalyse des phonologischen Arbeitsgedächtnisses. In: M. Hasselhorn, W. Schneider \& H. Marx (Hrsg.): Diagnostik von Lese-/Rechtschreibschwierigkeiten. Tests und Trends, N.F. Bd. 1. Jahrbuch der pädagogischpsychologischen Diagnostik (S. 167-181). Göttingen: Hogrefe.

Hasselhorn, M., Hille, B., Süß, U. Werner, I. \& Grube, D. (1995). Ist die Entwicklungsdysphasie die Folge von Entwicklungsstörungen im Arbeitsgedächtnis? Vortrag auf der 12. Tagung Entwicklungspsychologie vom 25.-29.9.1995.

Hasselhorn, M. \& Körner, K. (1997). Nachsprechen von Kunstwörtern: Zum Zusammenhang zwischen Arbeitsgedächtnis und syntaktischen Sprachleistungen bei Sechs- und Achtjährigen. Zeitschrift für Entwicklungspsychologie und Pädagogische Psychologie, 29, 212236.

Hasselhorn, M., Lingelbach, H. \& Gabert, A. (1991). Zur Entwicklung des Arbeitsgedächtnisses bei Schulkindern: Ist der phonetische Ähnlichkeitseffekt auf die Gedächtnisspanne wirklich altersabhängig? Zeitschrift für Entwicklungspsychologie und Pädagogische Psychologie, 13, 2, 148-158.

Hasselhorn, M. \& Seidler, U. (1999). Ist das „Nachsprechen von Kunstwörtern“ für Entwicklungsanalysen des phonologischen Arbeitsgedächtnisses geeignet? Vortrag auf der 14. Tagung Entwicklungspsychologie vom 13.-16. September 1999 in Fribourg (Schweiz).

Hasselhorn, M., Seidler-Brandler, U. \& Körner, K. (2000a). Ist das „Nachsprechen von Kunstwörtern" für die Entwicklungsdiagnostik des phonologischen Arbeitsgedächtnisses geeignet? In: M. Hasselhorn, W. Schneider \& H. Marx (Hrsg.): Diagnostik von Lese/Rechtschreibschwierigkeiten. Tests und Trends, N.F. Bd. 1. Jahrbuch der pädagogischpsychologischen Diagnostik(S. 119-133). Göttingen: Hogrefe.

Hasselhorn, M., Tiffin-Richards, M.C., Woerner, W., Banaschewski, T. \& Rothenberger, A. (2000b). Spielt der phonetische Speicher des Arbeitsgedächtnisses eine bedeutsame Rolle für die Differentialdiagnose von Lese-/Rechtschreibschwierigkeiten? Analysen zum „Kunstwörter-Nachsprechen“ bei Kindern mit LRS- und/oder HKS-Diagnose. In: M. Hasselhorn, W. Schneider \& H. Marx (Hrsg.): Diagnostik von Lese-/Rechtschreibschwierigkeiten. Tests 
und Trends, N.F. Bd. 1. Jahrbuch der pädagogisch-psychologischen Diagnostik(S. 149-165). Göttingen: Hogrefe.

Hier, D.B., LeMay, M., Rosenberger, P.B. \& Perlo, V. (1978). Developmental dyslexia: Evidence for a subgroup with reversed cerebral asymmetry. Archives of Neurology, 35, 9092.

Hennighausen, K., Remschmidt, H. \& Warnke, A. (1994). Visually evoked potentials in boys with developmental dyslexia. European Child and Adolescent Psychiatry, 3, 72-118.

Horn, W. (1969). Prüfsystem für Schul- und Bildungsberatung. P-S-B. Göttingen: Hogrefe.

Horwitz, B., Rumsey, J.M., Donohue, B.C. (1998). Functional connectivity of the angular gyrus in normal reading and dyslexia Proceedings of the National Academy of Sciences of the United States of America, 95, 8939-8944.

Hulme, C. (1984). Developmental differences in the effects of acoustic similarity on memory span. Developmental Psychology, 20, 650-652.

Imada, T., Fukuda, K., Kawakatsu, M., Masjiko, T., Odaka, K., Hayashi, M., Aihara, K. \& Kotani, M. (1993). Mismatch fields evoked by a rhythm passage. In: L. Deecke, C. Baumgartner, G. Stroink \& S.J. Williamson (Hrsg.): Advances in biomagnetism (S. 118119). Wien: $9^{\text {th }}$ International Conference on Biomagnetism.

Jääskeläinen I., Alho, K., Escera, K., Winkler, I., Sillanaukee, P. \& Näätänen, R. (1996). Effects of ethanol and auditory distraction on forced choice reaction in time. Alcohol, 13, 153-156.

Jack, A. (1990). Effects of instrumental music and environmental sounds on phonological short-term memory. Unpublished bachelor's thesis, University of Aberdeen, Aberdeen.

Jansen, H., Mannhaupt, G., Marx, H. \& Skowronek, H. (1998). Bielefelder Screening zur Früherkennung von Lese-/Rechtschreibschwierigkeiten (BISC). Göttingen: Hogrefe.

Jaramillo, M., Alku, P. \& Paavilainen, P. (1999). An event-related potential (ERP) study of duration changes in speech and non-speech sounds. NeuroReport, 10, 3301-3305.

Jones, D. (1993). Objects, streams, and threads of auditory attention. In: A. Baddeley \& L. Weiskrantz (Hrsg.): Attention: Selection, awareness, and control (S.87-104). Oxford: Clarendon Press.

Jones, D.M. \& Macken, W.J. (1993). Irrelevant tones produce an irrelevant speech effect: Implications for phonological coding in working memory. Journal of Experimental Psychology: Learning, Memory, and Cognition, 19, 369-381.

Jorm, A.F., Share, D.L., MacLean, R. \& Matthews, R. (1986). Cognitive factors at school entry predictive of specific reading retardation and general reading backwardness: A research note. Journal of Child Psychology and Psychiatry, 27, 45-54.

Kaufmann, W.E. \& Galaburda, A.M. (1989). Cerebrocortical microdysgenesis in neurologically normal subjects: A histopathologic study. Neurology, 39, 238-244. 
Kaukoranta, E., Sams, M., Hari, R., Hämäläinen, M. \& Näätänen, R. (1989). Reactions of auditory cortex to a change in tone duration. Hearing Research, 41, 15-22.

Klatte, M. \& Hellbrück, J. (1993). Der „Irrelevant Speech Effect“: Wirkungen von Hintergrundschall auf das Arbeitsgedächtnis. Zeitschrift für Lärmbekämpfung, 40, 91-98.

Klicpera, C. \& Gasteiger-Klicpera, B. (unter Mitarbeit von A. Schabmann) (1993). Lesen und Schreiben - Entwicklung und Schwierigkeiten. Die Wiener Längsschnittuntersuchungen über die Entwicklung, den Verlauf und die Ursachen von Lese-Schreibschwierigkeiten in der Pflichtschulzeit. Bern: Huber.

Klicpera, C. \& Gasteiger-Klicpera, B. (1995). Psychologie der Lese- und Schreibschwierigkeiten. Weinheim: Psychologie Verlags Union.

Klicpera, C. \& Gasteiger-Klicpera, B. (2001). Macht Intelligenz einen Unterschied? Rechtschreiben und phonologische Fertigkeiten bei diskrepanten und nichtdiskrepanten Lese/Rechtschreibschwierigkeiten. Zeitschrift für Kinder- und Jugendpsychiatrie und Psychotherapie, 29, 1, 37-49.

Kraus, N., McGee, T.J., Carrell, T.D. \& Sharma, A. (1995). Neurophysiologic bases of speech discrimination. Ear and Hearing, 16, 19-37.

Kraus, N., McGee, T.J., Sharma, A., Carrell, T.D. \& Nicol, T.G. (1992). Mismatch negativity event-related potential elicited by speech stimuli. Ear and Hearing, 13, 158-164.

Kujala, T., Karma, K., Čeponiene, R., Belitz, S., Turkkila, P., Tervaniemi, M. \& Näätänen, R. (2001). Plastic neural changes and reading improvement caused by audiovisual training in reading-impaired children. PNAS, 98, 18, 10509-10514.

Kujala, T., Myllyviita, K., Tervaniemi, M., Alho, K. Kallio, J. \& Näätänen, R. (2000). Basic auditory dysfunction in dyslexia as demonstrated by brain activity measurements. Psychophysiology, 37, 1-5.

Kujala, T. \& Näätänen, R. (2001). The mismatch negativity in evaluating central auditory dysfunction in dyslexia. Neuroscience and Biobehavioral Reviews, 25, 535-543.

Küspert, P. (1998). Phonologische Bewusstheit und Schriftspracherwerb: Zu den Effekten vorschulischer Förderung der phonologischen Bewusstheit auf den Erwerb des Lesens und Rechtschreibens. Frankfurt a.M.: Lang.

Küspert, P. \& Schneider, W. (1999; 2003). Hören, lauschen, lernen. Sprachspiele für Kinder im Vorschulalter. Würzburger Trainigsprogramm zur Vorbereitung auf den Erwerb der Schriftsprache. 4. Auflage (2003). Göttingen. Vandenhoeck \& Ruprecht.

Landauer, T.K. (1962). Rate of implicit speech. Perceptual and Motor Skills, 15, 646.

Landerl, K. \& Wimmer, H. (1994). Phonologische Bewusstheit als Prädiktor für Lese- und Schreibfertigkeiten in der Grundschule. Zeitschrift für Pädagogische Psychologie, 8, 153164. 
Lang, H.A., Nyrke, T., Ek, M., Aaltonen, O., Raimo, I. \& Näätänen, R. (1990). Pitch discrimination performance and auditory event-related potentials. In: C.H.M. Brunia, A.W.K. Gaillard, A. Kok, G. Mulder \& M.N. Verbaten (Hrsg.): Psychophysiological brain research (Bd. 1, S. 294-298). Tilburg: Tilburg University Press.

Leppänen, P.H.T., Richardson, U., Pihko, E., Eklund, K.M., Guttorm, T.K., Aro, M. \& Lyytinen, H. (2002). Brain responses to changes in speech sound durations differ between infants with and without familial risk for dyslexia. Developmental Neuropsychology, 22, 1, 407-422.

Levänen, S, Ahonen, A., Hari, R., McEvoy, L. \& Sams, M. (1996). Deviant auditory stiuli activate human left and right auditory cortex differently. Cerebral Cortex, 6, 288-296.

Liberman, I.Y. \& Shankweiler, D., Orlando, C., Harris, K.S. \& Berti, F.B. (1971). Letter confusions and reversals of sequence in the beginning reader: Implications for Orton's theory of developmental dyslexia. Cortex, 7, 127-142.

Linder, M. (1951). Über Legasthenie (spezielle Leseschwäche). Zeitschrift für Kinderpsychiatrie, 17, 97.

Linder, M. \& Grissemann, H. (1996). Zürcher Lesetest. Göttingen. Hogrefe.

Livingstone, M.S., Rosen, C.D., Dislane, F.W. \& Galaburda, A.M. (1991). Physiological and anatomical evidence for a magnocellular defect in developmental dyslexia. Proceedings of the National Academy of Sciences of the United States of America, 88, 7943-7947.

Locke, J.L. (1997). A theory of neurolinguistic development. Brain and Language, 58, 265-326.

Logie, R.H. (1995). Visual-spatial working memory. Hove: Lawrence Erlbaum Associates.

Lounasmaa, O.V., Hari, R., Joutsiniemi, S.-L. \& Hämäläinen, M. (1989). Multi-SQUID recordings of human cerebral magnetic fields may give information about memory processes. Europhysics Letters, 9, 603-608.

Lubs, H.A., Smith, S., Kimberling, W., Pennington, B., Gross-Glenn, K. \& Duara, R. (1988). Dyslexia subtypes: Genetics, behavior, and brain imaging. In: F. Plum (Hrsg.): Language, Communication and the Brain. New York: Raven Press.

Lundberg, I., Frost, J. \& Peterson, O. (1988). Effects of an extensive training program for stimulating phonological awareness in preschool children. Reading Research Quarterly, 23, 263-284.

Lundberg, I., Olofsson, A., Wall, S. (1980). Reading and spelling skills in the first school years predicted from phonemic awareness skills in kindergarten. Scandinavian Journal of Psychology, 21, 159-173.

Lyytinen, H., Blomberg, A.P. \& Näätänen, R. (1992). Event-related potentials and autonomic responses to a change in unattended auditory stimuli. Psychophysiology, 29, 523-534.

Manis, F.R., Seidenberg, M.S., Doi, L.M., McBride-Chang \& Peterson, A. (1996). On the basis of two subtypes of developmental dyslexia. Cognition, 58, 157-195. 
Marshall, C.M., Snowling, M.J. \& Bailey, P.J. (2001). Rapid auditory processing and phonological ability in normal readers and readers with dyslexia. Journal of Speech, Language, and Hearing Research, 44, 925-940.

Marx, H. (1997). Erwerb des Lesens und Rechtschreibens. In: F.E. Weinert \& A. Helmke (Hrsg.): Entwicklung im Grundschulalter (S. 83-138). Weinheim: Psychologie Verlags Union.

Marx, P. (1998). Zur Erfassung der phonologischen Informationsverarbeitung im Grundschulalter. Weinheim: Psychologie Verlags Union.

Marx, P., Weber, J.-M. \& Schneider, W. (2001),. Legasthenie versus allgemeine Lese/Rechtschreibschwäche. Ein Vergleich der Leistungen in der phonologischen und visuellen Informationsverarbeitung. Zeitschrift für Pädagogische Psychologie, 15, 2, 85-98.

Maugham, B. (1995). Annotation: Long-term outcomes of developmental reading problems. Journal of Child Psychology and Psychiatry, 36: 357-371.

Maugham, B., Gray, G. \& Rutter, M. (1985). Reading retardation and antisocial behavior: A follow-up into employment. Journal of Child Psychology and Psychiatry, 26: 741-758.

Mayringer, H. \& Wimmer, H. (1999). Pseudoname learning by german-speaking children with dyslexia: evidence for a phonological learning deficit. Journal of Experimental Child Psychology, 75, 116-113.

McGee, R., Share, D., Moffitt, T.E., Williams, S. \& Silva, R.A. (1988). Reading disability, behavior problems and juvenile delinquency. In: Saklofske, D.H., Eysenck, S.B.G. (Hrsg.): International perspectiveness. London: Holder \& Stoughton, 357-390.

Mody, M. (1993). Bases of reading impairment in speech perception: A deficit in rate of auditory processing or in phonological coding? Unpublished doctoral dissertation. City University of New York.

Mody, M., Studdert-Kennedy, M. \& Brady, S. (1995). Speech perception deficits in poor readers: Auditory processing or phonological coding? Journal of Experimental Child Psychology, 64, 199-231.

Morais, J., Cary, L., Alegria, J. \& Bertelson, P. (1979). Does awareness of speech as a sequence of phonemes arise spontaneously? Cognition, 7, 323-331.

Morgan, W.P. (1896). A case of congenital wordblindness. British Medical Journal, 2, 13-78.

Morris, R.D., Stuebing, K.K., Fletcher, J.M., Shawitz, S.E., Lyon, G.R., Shankweiler, D.P., Katz, L., Francis, D.J. \& Shaywitz, B.A. (1998). Subtypes of reading disability: Variability around a phonological core. Journal of Educational Psychology, 90, 347-373.

MacLean, M., Bryant, P.E. \& Bradley, L. (1987). Rhymes, nursery rhymes and reading in early childhood. Merrill-Palmer Quarterly, 33, 255-282.

Mottier, G. (1951). Der Mottier-Test. Über Untersuchungen zur Sprache lesegestörter Kinder. Folia Phoniatrica, 3, 170 - 177. 
Näätänen, R. (1992). Attention and brain function. Hillsdale, NJ: Lawrence Erlbaum.

Näätänen, R. (1995). The mismatch negativity: A powerful tool for cognitive neuroscience. Ear and Hearing, 16, 6-8.

Näätänen, R. \& Alho, K. (1995). Mismatch negativity - a unique measure of sensory auditory processing in audition. International Journal of Neuroscience, 80, 317-337.

Näätänen, R., Gaillard, A.W.K. \& Mäntysalo, S. (1978). Early selective attention effect on evoked potential reinterpreted. Acta Psychologica, 42, 313-329.

Näätänen, R., Jiang, D., Lavikainen, J., Reinikainen, K. \& Paavilainen, P. (1993a). Eventrelated potentials reveal a memory trace for temporal features. NeuroReport, 5, 310-312.

Näätänen, R., Paavilainen, P. \& Reinikainen, K. (1989). Do event-related potentials to infrequent decrements in duration of auditory stimuli demonstrate a memory trace in man? Neuroscience Letters, 107, 347-352.

Näätänen, R., Paavilainen, P., Alho, K., Reinikainen, K. \& Sams, M. (1987). The mismatch negativity to intensity changes in an auditory stimulus sequence. in: R. Johnson Jr., J.W. Rohrbaugh \& R. Parasuraman (Hrsg.): Current trends in event-related potential research (S. 125-131). Amsterdam: Elsevier.

Näätänen, R., Schröger, E., Karakas, S., Tervaniemi, M. \& Paavilainen, P. (1993b). Development of a memory trace for a complex sound in the human brain. NeuroReport, 4 , 503-506.

Näätänen, R. \& Winkler, I. (1999). The concept of auditory stimulus representation in cognitive neuroscience. Psychological Bulletin, 125, 6, 826-859.

Näslund, J.C. \& Schneider, W. (1996). Kindergarten letter knowledge, phonological skills, and memory process: Relative effects on early literacy. Journal of Experimental Child Psychology, 62, 30-59.

Naylor, C.E., Felton, R.H. \& Wood, F.B. (1990). Adult outcome in developmental dyslexia. In: Pavlidis, G.T. (Hrsg.): Perspectives on Dyslexia. Vol. 2. London: Wiley, 215-229.

Neisser, U. (1967). Cognitive Psychology. New York: Appleton-Century-Crofts.

Nordby, H., Roth, W.T. \& Pfefferbaum, A. (1988). Event-related potentials to time-deviant and pitch-deviant tones. Psychophysiology, 25, 249-261.

Öhmann, A. (1979). The orienting response, attention and learning: An information-processing perspective. In: H.D. Kimmel, E.H. van Olst \& J.F. Orlebeke (Hrsg.): The orienting reflex in humans (S. 443-471). Hillsdale, NJ: Erlbaum.

Öhmann, A. (1992). Orienting and attention: Preferred preattentive processing of potentially phobic stimuli.In: B.A. Campbell, R. Richardson \& H. Hayne (Hrsg.): Attention and information processing in infants and adults: Perspectives from human and animal research (S. 263-295). Hillsdale, NJ: Erlbaum.

Orton, S.T. (1937). Reading, writing, and speech problems in children. New York: Norton. 
Orvarschel, H., Puig-Antich, J. (1987). Schedule for Affective Disorder in Schizophrenia for School Age Children: K-SADS-E. $4^{\text {th }}$ ed. Pittsburgh: Western Psychiatric Institute.

Paavilainen, P., Alho, K., Reinikainen, K., Sams, M. \& Näätänen, R. (1991). Righthemisphere dominance of different mismatch negativities. Electroencephalography and Clinical Neuroscience, 78, 466-479.

Paavilainen, P., Karlsson, M-L., Reinikainen, K. \& Näätänen, R (1989). Mismatch negativity to change in spatial location of an auditory stimulus. Electroencephalography and Clinical Neurophysiology, 73, 129-141.

Ranschburg (1916). Die Leseschwäche (Legasthenie) und Rechenschwäche (Arithmasthenie) der Schulkinder im Licht des Experimentes. Berlin. [zitiert nach Golkenrath, 1984, S. 7 ff.]

Rathenow, P. (1979). Westermann Rechtschreibtest 4/5. Braunschweig: Westermann.

Rathenow, P., Laupenmühlen, D. \& Vöge, J. (1981). Westermann Rechtschreibtest 6+. Braunschweig: Westermann.

Rayner, K., Pollatsek, A. \& Bilsky, A.B. (1995). Can temporal processing deficit account for dyslexia? Psychonomic Bulletin and Review, 2, 501-507.

Read, C., Zhang, Y.-F., Nie, H.-Y. \& Ding, B.-Q. (1986). The ability to manipulate speech sounds depends on knowing alphabetic writing. Cognition, 24, 31-45.

Reed, M.A. (1989). Speech perception and the discrimination of breif auditory cues in reading disabled children. Journal of Experimental Child Psychology, 48, 270-292.

Remschmidt, H., \& Walter, R. (1990). Psychische Auffälligkeiten bei Schulkindern. Göttingen: Hogrefe.

Rutter, M., Tizard, J., Yule, W., Graham, P. \& Whitmore, K. (1976). Isle of Wight Studies 1964 - 1974. Psychological Medicine, 6, 313-332.

Sandridge, S.A. \& Boothroyd, A. (1996). Using naturally produced speech to elicit the mismatch negativity. Journal of the American Academy of Audiology, 7, 105-112.

Saß, H., Wittchen, H.-U., Zandig, M. (Hrsg.) (1998). Diagnostisches und Statistisches Manual Psychischer Störungen DSM IV. 2. Auflage. Göttingen: Hogrefe.

Satz, P., Rardin, D \& Ross, J. (1971). An evaluation of a theory of specific developmental dyslexia. Child Development, 42, 2009-2021.

Sawyer, D.J. \& Fox, B.J. (Hrsg.) (1991). Phonological awareness in reading: The evolution of current perspectives. New York: Springer.

Schneider, W. (1997). Rechtschreiben und Rechtschreibschwierigkeiten. in F.E. Weinert (Hrsg.): Enzyklopädie der Psychologie. Serie Pädagogische Psychologie, Bd. 3: Psychologie des Unterrichts und der Schule(S. 327-363). Göttingen: Hogrefe.

Schneider, W. \& Näslund, J.C. (1993). The impact of early metalinguistic competencies and memory capacity on reading and spelling in elementary school: Results of the Munich 
Longitudinal Study on the Genesis of Individual Competencies (LOGIC). European Journal of Psychology of Education, 8, 273-288.

Schneider, W., Visé, M, Reimers, P. \& Blaesser, B (1994). Auswirkungen eines Trainings der sprachlichen Bewusstheit auf den Schriftspracherwerb in der Schule. Zeitschrift für Pädagogische Psychologie, 8, 177-188.

Schröger, E. (1996). A neural mechanism for involuntary attention shifts to changes in auditory stimulus stimulation. Journal of Cognitive Neuroscience, 8, 527-539.

Schröger, E. \& Wolff, C. (1996). Mismatch response of the human brain to changes in sound location. NeuroReport, 7, 3005-3008.

Schulte-Körne, G., Deimel, W., Bartling, J. \& Remschmidt H. (1998b). Die Bedeutung der auditiven Wahrnehmung der phonologischen Bewusstheit für die Lese-/Rechtschreibschwäche. Sprache - Stimme - Gehör, 22, 24-29.

Schulte-Körne, G., Deimel, W., Bartling, J. \& Remschmidt H. (1998c). Auditory processing and dyslexia: evidence for a specific speech processing deficit. NeuroReport, 9, 337-340.

Schulte-Körne, G., Deimel, W., Bartling, J. \& Remschmidt H. (1999a). Pre-attentive processing of auditory patterns in dyslexic human subjects. Neuroscience Letters, 276, 41-44.

Schulte-Körne, G., Deimel, W., Bartling, J. \& Remschmidt H. (1999b). The role of phonological awareness, speech perception, and auditory temporal processing for dyslexia. European Child \& Adolescent Psychiatry, 8, Suppl. 3, 28-34.

Schulte-Körne, G., Deimel, W., Müller, K., Gutenbrunner, C. \& Remschmidt, H. (1996). Familial aggregation of spelling disability. Journal of Child Psychology and Psychiatry, 37, 817-822.

Schulte-Körne, G., Deimel, W. \& Remschmidt H. (2001). Zur Diagnostik der Lese-/Rechtschreibstörung. Zeitschrift für Kinder- und Jugendpsychiatrie und Psychotherapie, 29, 2 , 113-116.

Schulte-Körne, G., Grimm, T., Nöthen, M.M., Müller-Myhsok, B., Cichon, S., Vogt, I.R., Propping, P. \& Remschmidt, H. (1998a). Evidence for linkage of spelling disabilty to chromosome 15. American Journal of Human Genetics, 63, 279-282.

Schulte-Körne, G., Remschmidt, H. \& Hebebrand, J. (1993). Zur Genetik der Lese/Rechtschreibschwäche. Zeitschrift für Kinder- und Jugendpsychiatrie, 21, 242-252.

Shannon, R.V., Zeng, F.-G., Kamath, V., Wygnonski, J. \& Ekelid, M. (1995). Speech recognition with primarily temporal cues. Science, 270, 303-304.

Shaywitz, S.E. (1996, November). Dyslexia. Scientific American, 98-104.

Siegel, L.S., Share, D. \& Geva, E. (1995). Evidence for superior graphic skills in dyslexics. Psychological Science, 6, 250-254. 
Silva, P.A., McGee, R. \& Williams, S. (1985). Some characteristics of 9-year old boys with general reading backwardness or specific reading retardation. Journal of Child Psychology and Psychiatry, 26, 407-421.

Snowling, M.J. (2000). Dyslexia. 2. Ausg. Oxford: Blackwell Publishers Ltd.

Stanovich, K.E., Siegel, L.S. \& Gottardo, A. (1997). Converging evidence for phonological and surface subtypes of reading disability. Journal of Educational Psychology, 89, 114-127.

Stanovich, K.W. (1988). The right and wrong places to look for the cognitive locus of reading disability. Annals of Dyslexia, 38, 154-177.

Stanovich, K.W. (1991). Changing models of reading and reading acquisition. In: L. Rieben \& C. Perfetti (Hrsg.): Learning to read: Basic research and its implications (pp. 19-31). Hillsdale: Erlbaum.

Steffens, M.L., Eilers, R.E., Gross-Glenn, K. \& Jallad, B. (1992). Speech perception in adult subjects with familial dyslexia. Journal of Speech and Hearing Research, 35, 192-200.

Stevenson, J., Graham, P., Fredman, G. \& McLoughlin, V. (1987). A twin study of genetic influences on reading and spelling ability and disability. Journal of Child Psychology and Psychiatry, 28, 229-247.

Strehlow, U., Kluge, R., Müller, H. \& Haffner, J. (1992). Der langfristige Verlauf der Legasthenie über die Schulzeit hinaus. Katamnesen aus einer kinderpsychiatrischen Ambulanz. Zeitschrift für Kinder- und Jugendpsychiatrie 20, 254-265.

Studdert-Kennedy, M. \& Mody, M. (1995). Auditory temporal perception deficits in the reading impaired: A critical review of the evidence. Psychonomic Bulletin \& Review, 2, 4, 508-514.

Swan, D. \& Goswami, U. (1997). Phonological awareness deficits in developmental dyslexia and the phonological representations hypothesis. Journal of Experimental Child Psychology, $66,18-41$.

Talcott, J.B., Witton, C., McClean, M., Hansen, P.C. Rees, A., Green, G.G.R. \& Stein, J.F. (1999). Can sensitivity to auditory frequency modulation predict children's phonological and reading skills? NeuroReport, 10, 2045-2050.

Tallal, P. (1980). Auditory temporal perception, phonics, and reading disabilities in children. Brain and Language, 9, 182-198.

Tallal, P., Miller, S.L., Jenkins, W.M. \& Merzenich, M.M. (1997). The role of temporal processing in developmental language-based learning disorders: Research and clinical implications. In: B.A. Blachman (Hrsg.): Foundations of reading acquisition and dyslexia ( S. 49-66). Hillsdale, NJ: Erlbaum.

Taylor, H.G., Satz, P. \& Friel, J. (1979). Developmental dyslexia in relation to other childhood reading disorders: Significance and clinical utility. Reading Research Quarterly, 15, 84-101. 
Tewes, U., Rossmann, P. \& Schallenberger U. (1999). Hamburg-Wechsler-Intelligenztest für Kinder - Dritte Auflage. HAWIK-III. Göttingen: Huber.

Tiitinen, H., May, P., Reinikainen, K. \& Näätänen, R. (1994). Attentive novelty detection in humans is governed by pre-attentive sensory memory. Nature, 372, 90-92.

Tiitinen, H., Sinkkonen, J., May, P. \& Näätänen, R. (1994). The auditory transient 40-Hz response is insensitive to changes in stimulus features. NeuroReport, 6, 190-192.

Treiman, R. (1991). Phonological awareness and its roles in learning to read and to spell. In: D.J. Sawyer \& B.J. Fox (Hrsg.): Phonological awareness in reading: The evolution of current perspectives (pp. 159-189). New York: Springer.

Wagner, R.K. (1986). Phonological processing abilities and reading: Implications for disabled readers. Journal of Learning Disabilities, 19, 623-630.

Wagner, R.K. \& Torgesen, J.K. (1987). The nature of phonological processing and ist causal role in the acquisition of reading skills. Psychological Bulletin, 101, 192-212.

Wagner, R.K., Torgesen, J.K. \& Rashotte, C. (1994). Development of reading-related phonological processing abilities: new evidence of bidirectional causality from a latent variable longitudinal study. Developmental Psychology, 30, 1, 73-87.

Wagner, R.K., Torgesen, J.K., Rashotte, C.A. Hecht, S.A. Berker, T.A., Burgess, S.R. Donahue, J. \& Garon, T. (1997). Changing relations between phonological processing abilities and word-level reading als children develop from beginning to skilled readers: A 5year longitudinal study. Developmental Psychology, 33, 468-479.

Warnke, A. (1990). Legasthenie und Hirnfunktion. Neuropsychologische Befunde zur visuellen Informationsverarbeitung. Bern: Huber.

Warnke, A. \& Remschmidt, H. (1992). Visual information processing in developmentally dyslexic boys: A neuropsychological study. In: Remschmidt, H., Schmidt, M.H. (Hrsg.): Developmental Psychopathology. Göttingen: Hogrefe.

Warnke, A. \& Wewetzer, Ch. (1997). Legasthenie: Definition und Psychopathologie. Zeitschrift praktische Augenheilkunde und augenärztliche Fortbildung, 18, 94-102.

Watson, B.U. \& Miller, T.K. (1993). Auditory perception, phonological processing, and reading ability/disability. Journal of Speech and Hearing Research, 336, 850-863.

Weinert, F.E. \& Schneider, W. (Hrsg.) (1987). The Munich Longitudinal Study on the Genesis of Individual Competencies (LOGIC), Report No. 2: Documentation of assessment procedures used in waves one to three. Technical Report, Munich: Max Planck Institute for Psychological Research.

Weinert, F.E. \& Schneider, W. (Hrsg.) (1992). The Munich Longitudinal Study on the Genesis of Individual Competencies (LOGIC), Report No. 8: Assessment procedures and results of wave five. Technical Report, Munich: Max Planck Institute for Psychological Research. 
Weltgesundheitsorganisation (1993). Internationale Klassifikation psychischer Störungen. ICD 10. Kapitel V. Klinisch - diagnostische Leitlinien. Dilling, H., Mombour, W., Schmidt, M.H. (Hrsg.). 2. Auflage. Göttingen: Huber.

Werker, J.F. \& Tees, R.C. (1987). Speech perception in severely disabled and average reading children. Canadian Journal of Psychology, 41, 48-61.

Williams, R.W. \& Rakic, P. (1988). Eliminations of neurons from the rhesus monkey's lateral geniculate nucleus during development. The Journal of Comparative Neurology, 272, 424436.

Winkler, I., Kujala, T., Tiitinen, H., Sivonen, P., Alku, P., Lehtokoski, A., Czigler, I. Csépe, V., Ilmoniemi, R.J. \& Näätänen, R. (1999). Brain responses reveal the learning of foreign language phonemes. Psychophysiology, 36, 638-642.

Winkler, I. \& Schröger, E. (1995). Neural representation for the temporal structure of sound patterns. NeuroReport, 6, 690-694.

Winkler, I., Tervaniemi, M., Schröger, E., Wolff, C. \& Näätänen, R. (1998). Pre-attentive processing of auditory spatial information: Electrophysiological evidence from human subjects. Neuroscience Letters, 242, 49-52.

Wolf, M. (1991). Naming speed and reading: The contribution of the cognitive neurosciences. Reading Research Quarterly, 26, 123-141.

Wolf, M., \& Bowers, P.G. (1999). The double-deficit hypothesis for the development dyslexia. Journal of Educational Psychology, 91, 415-438.

Wolf, M. \& Obregon M. (1992). Early naming deficits, developmental dyslexia, and a specific deficit hypothesis. Brain and Language, 42, 219-247. 


\title{
9. ANHANG
}

\author{
ANHANGSVERZEICHNIS
}

Anhang A:

Protokollbogen

ii - ix

Anhang B:

Datenblatt mit Legende

$\mathrm{x}-\mathrm{xi}$

Anhang C:

Kovarianz- /Korrelationsmatrix

xii 
ANHANG A:

PROTOKOLLBOGEN DER KOGNITIONSPSYCHOLOGISCHEN TESTS

\section{WORTSPANNE}

Vorgabe: 1 Wort/Sekunde

\begin{tabular}{|l|l|l|}
\hline i. & Haus - Ball - Pilz & \\
\hline 2a & Stern - Topf & \\
\hline 2b & Fisch - Baum & \\
\hline 3a & Ball - Fisch - Schuh & \\
\hline 3b & Fisch - Stern - Baum & \\
\hline 4a & Pilz - Ball - Baum - Topf & \\
\hline 4b & Schuh - Fisch - Haus - Stern & \\
\hline 5a & Topf - Haus - Baum - Pilz - Ball & \\
\hline 5b & Haus - Fisch - Stern - Schuh - Pilz & \\
\hline $6 a$ & Baum - Pilz - Ball - Stern - Topf - Fisch & \\
\hline $6 b$ & Stern - Fisch - Schuh - Haus - Topf - Ball & \\
\hline $7 a$ & Pilz - Schuh - Topf - Stern - Fisch - Baum -Haus & \\
\hline $7 b$ & Fisch - Ball - Pilz - Haus - Schuh - Stern - Baum & \\
\hline 8a & Ball - Pilz - Schuh - Baum - Topf - Haus - Fisch - Stern & \\
\hline 8b & Schuh - Fisch - Baum - Stern - Ball - Haus - Pilz - Topf & \\
\hline & GEDÄCHTNISSPANNE: & \\
\hline
\end{tabular}




\section{MOTTIER}

Normen Mottier

\begin{tabular}{|l|l|c|c|c|c|c|c|c|}
\hline \multicolumn{2}{|c|}{ Alter } & $7 \mathrm{~J}$. & $8 \mathrm{~J}$. & $9 \mathrm{~J}$. & $10 \mathrm{~J}$. & $11 \mathrm{~J}$. & $12 \mathrm{~J}$. & $13-16 \mathrm{~J}$. \\
\hline 4 & normal & 22 & 23 & $23-24$ & 24 & 25 & 25 & 26 \\
\hline 3 & red. & $16-18$ & $17-19$ & $18-20$ & $19-21$ & $20-22$ & $20-22$ & $21-22$ \\
\hline 2 & stk. red. & $12-15$ & $13-16$ & $14-17$ & $15-18$ & $16-19$ & $17-19$ & $18-20$ \\
\hline 1 & sehr stk. red. & $<12$ & $<13$ & $<14$ & $<15$ & $<16$ & $<17$ & $<18$ \\
\hline
\end{tabular}

\section{MOTTIER-KUNSTWORTE}

\begin{tabular}{|c|c|c|}
\hline \multicolumn{2}{|c|}{ CD } & \multirow[t]{2}{*}{ Pkte } \\
\hline \multicolumn{2}{|r|}{ rela } & \\
\hline 2 & \multicolumn{2}{|l|}{ noma } \\
\hline 3 & godu & \\
\hline 4 & mera & \\
\hline 5 & luri & \\
\hline 6 & limo & \\
\hline 7 & kapeto & \\
\hline 8 & giboda & \\
\hline$\overline{9}$ & lorema & \\
\hline 10 & tokipa & \\
\hline 11 & dugabe & \\
\hline 12 & nomari & \\
\hline 13 & pikatura & \\
\hline 14 & gabodila & \\
\hline 15 & monalura & \\
\hline$\overline{16}$ & topakimo & \\
\hline 17 & debagusi & \\
\hline 18 & relomano & \\
\hline
\end{tabular}

\begin{tabular}{|l|l|l|}
\hline 19 & katopinafe & \\
\hline 20 & gebidafino & \\
\hline 21 & ronamelita & \\
\hline 22 & tapikusawe & \\
\hline 23 & degobesaro & \\
\hline 24 & muralenoka & \\
\hline 25 & pekatorisema & \\
\hline 26 & dagobilaseta & \\
\hline 27 & leraminofeko & \\
\hline 28 & kapotilafesa & \\
\hline 29 & bigadonafera & \\
\hline 30 & nomalirakosa & \\
\hline GES & & \\
\hline
\end{tabular}




\section{WÜRZBURGER TESTBATTERIE}

\section{$\underline{\text { Vokalersetzung }}$}

$\mathrm{a} \rightarrow \mathrm{i}$; falsch: Im Falle mehrerer ,,a“ ist das Wort falsch, wenn nicht alle „a“ korrekt ersetzt wurden

(„Pizzia“) oder wenn die „¡““ - angrenzenden Laute verkehrt sind. Der Wortrest ansonsten darf falsch sein.

\begin{tabular}{|c|c|c|}
\hline Wand & Mathematik & Klasse \\
\hline Kran & Klara & Andreas \\
\hline Pizza & Kartoffel & lachen \\
\hline Ananas & Namenstag & Anna \\
\hline Fußball & Kamm & Handballmannschaft \\
\hline$\overline{\text { GESAMT }}$ & & \\
\hline
\end{tabular}

Restwortbestimmung

Der ganze Restwort muß korrekt wiedergegeben werden.

\begin{tabular}{|c|c|c|}
\hline Wal - al & Schlucken - lucken & omta - mta \\
\hline Trick - rick & Haus - aus & frasi - rasi \\
\hline Pferd - ferd & Pflaume - flaume & eglu - glu \\
\hline Ehre - re & groß - roß & bib - ib \\
\hline Knall - nall & Stimme - timme & spredo - predo \\
\hline GESAMT & & \\
\hline
\end{tabular}

Phonemvertauschung

Vertauschen der ersten beiden Laute. Richtig: Wenn die ersten beiden Laute korrekt vertauscht wurden, obwohl der Wortrest nicht mehr korrekt ist.

\begin{tabular}{|c|c|c|}
\hline Radio - ardio & Klasse - lkasse & elbi - lebi \\
\hline rot - ort & Igel - giel & serog - esrog \\
\hline Arbeit - rabeit & Ente - nete & raltu - arltu \\
\hline Tiger - itger & Löwe - ölwe & artali - ratali \\
\hline Ordnung - rodnung & Burg - ubrg & ekaut - keaut \\
\hline GESAMT & & \\
\hline
\end{tabular}




\section{$\underline{\text { Klassifikation }}$}

A: Der in der Reihe unterschiedliche Anfangslaut soll identifiziert werden.

B: Der in der Reihe unterschiedliche Endlaut soll identifiziert werden.

\begin{tabular}{|l|l|l|l|l|l|}
\hline A & fem - tar -fur -fap & & B & zoss - ress - poss - reff & \\
\hline A & gisch - gon - kik - gat & & B & Stern - Schirm - Stein - Bein & \\
\hline A & Kopf - Turm - tief - Trick & & B & Frank - Hand - Hemd - Sand & \\
\hline A & tosch - dup - tap - tus & & B & track - klott - dock - seck & \\
\hline A & Fisch - Schiff - frech - Vogel & & B & Teich - Deich - reich - Fleisch & \\
\hline A & mib - moch - mog - nog & & B & rung - spling - zunk - feng & \\
\hline & GESAMT: & & &
\end{tabular}

\section{LESETEST}

Bearbeitung entsprechend der altersgemäßen Anweisung:
2., 3. $K l$.:
WL $1+2$, evtl. Einzellaute
$L A 1+2+3$
4. $K l$ :
$W L 1+2+3$
$L A 3+4+5$
5. $\mathrm{Kl}$
$W L 1+2+3$
LA $3+4+5$
6. Kl.(evtl sim. 5. Kl.) WL 3:
LA $3+4+5+6$ 
5. PET

\begin{tabular}{|c|c|c|c|}
\hline & $\mathrm{CD}$ & $\underline{\mathbf{L V}}$ & \\
\hline $\mathrm{i}$ & 1 & Bsp.: Fisch & ---- \\
\hline 1 & 2 & Schuh & \\
\hline 2 & 3 & Eis & \\
\hline 3 & 4 & Uhr & \\
\hline 4 & 5 & Kind & \\
\hline 5 & 6 & Schiff & \\
\hline 6 & 7 & $\mathrm{Nu} ß$ & \\
\hline 7 & 8 & Maus & \\
\hline 8 & 9 & Zug & \\
\hline 9 & 10 & Zahn & \\
\hline 10 & 11 & alt & \\
\hline 11 & 12 & See & \\
\hline 12 & 13 & Kuh & \\
\hline 13 & 14 & Schnee & \\
\hline 14 & 15 & Rauch & \\
\hline 15 & 16 & Reis & \\
\hline 16 & 17 & Milch & \\
\hline 17 & 18 & müde & \\
\hline 18 & 19 & dick & \\
\hline 19 & 20 & Baum & \\
\hline 20 & 21 & Mond & \\
\hline 21 & 22 & Schirm & \\
\hline 22 & 23 & Radio & \\
\hline 23 & 24 & Feder & \\
\hline 24 & 25 & Garten & \\
\hline $\mathrm{i}$ & 26 & $\mathrm{za}$ & \\
\hline 25 & 27 & lek & \\
\hline
\end{tabular}

\begin{tabular}{|l|l|l|l|}
\hline 26 & 28 & vum & \\
\hline 27 & 29 & nast & \\
\hline 28 & 30 & spak & \\
\hline 29 & 31 & tapik & \\
\hline 30 & 32 & opasto & \\
\hline 31 & 33 & noride & \\
\hline 32 & 34 & adelmat & \\
\hline 33 & 35 & soberno & \\
\hline & & GES & \\
\hline
\end{tabular}

\begin{tabular}{|c|c|c|}
\hline 15 & 50 & Polizist \\
\hline 16 & 51 & Teppich \\
\hline 17 & 52 & Straßenbahn \\
\hline 18 & 53 & Bademantel \\
\hline 19 & 54 & Nachtigall \\
\hline 20 & 55 & Lesebuch \\
\hline 21 & 56 & Hausdach \\
\hline 22 & 57 & Kaufmann \\
\hline 23 & 58 & Dachdecker \\
\hline 24 & 59 & Tischtennisball \\
\hline 25 & 60 & Aufzug \\
\hline 26 & 61 & Telefonbuch \\
\hline 27 & 62 & Regenrinne \\
\hline 28 & 63 & Luftballon \\
\hline 29 & 64 & $\begin{array}{l}\text { Bügeleisen / Lügereien / } \\
\text { Hügelreihen }\end{array}$ \\
\hline 30 & 65 & $\begin{array}{l}\text { Sandkasten / } \\
\text { Wandkasten }\end{array}$ \\
\hline 31 & 66 & Liegestuhl \\
\hline 32 & 67 & Staubsauger \\
\hline 33 & 68 & Schuhputzzeug \\
\hline 34 & 69 & Plattenspieler \\
\hline 35 & 70 & $\begin{array}{l}\text { Schreibmaschine / } \\
\text { Eismaschine / } \\
\text { Teigmaschine }\end{array}$ \\
\hline 36 & 71 & Bordsteinkante \\
\hline & & GES \\
\hline
\end{tabular}

\begin{tabular}{|l|l|l|l|}
\hline \multicolumn{2}{|c|}{ CD } & \multicolumn{2}{c|}{ WE } \\
\hline 1 & 36 & Schokolade & \\
\hline 2 & 37 & Flugzeug & \\
\hline 3 & 38 & Limonade & \\
\hline 4 & 39 & Eisschrank & \\
\hline 5 & 40 & Spaghetti & \\
\hline 6 & 41 & Fingernagel & \\
\hline 7 & 42 & Kaffekanne & \\
\hline 8 & 43 & Sonnenschein & \\
\hline 9 & 44 & Teelöffel & \\
\hline 10 & 45 & Krokodil & \\
\hline 11 & 46 & Osterhase & \\
\hline 12 & 47 & Zeitung & \\
\hline 13 & 48 & Badewanne & \\
\hline 14 & 49 & Elefant / elegant & \\
\hline
\end{tabular}


6. WORTFLÜSSIGKEITSTEST

pro Buchstabe jeweils $1 \mathrm{~min}$

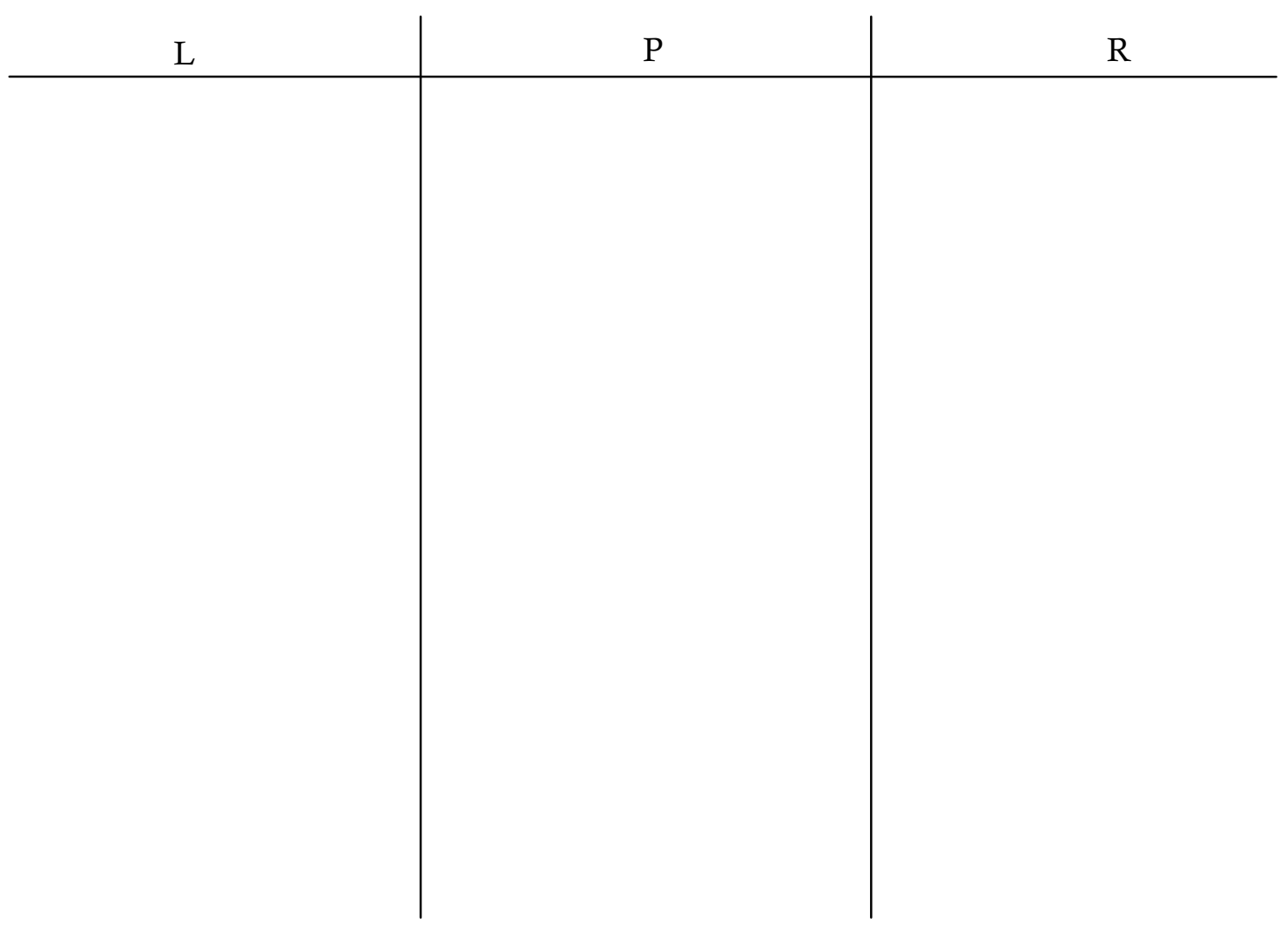

Gesamt [L]:

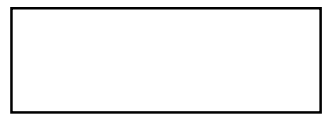

Gesamt [P]:

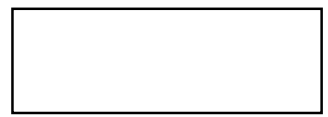

Gesamt [R]:

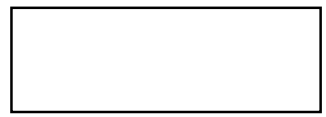

Gesamt $[L, P, R]$ :

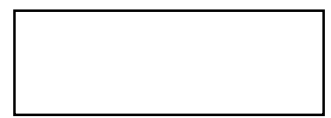




\section{RECHTSCHREIBTEST Für jedes Alter separat}

\section{KUNSTWÖRTER NACHSPRECHEN}

\begin{tabular}{|l|l|l|l|}
\hline & CD & & richtig? \\
\hline i & 31 & bralzen & \\
\hline i & 32 & bralzen & \\
\hline i & 33 & gliebunade & \\
\hline i & 34 & franulich & \\
\hline 1 & 35 & Werulater & \\
\hline 2 & 36 & Mattau & \\
\hline 3 & 37 & wuralten & \\
\hline 4 & 38 & Geserabung & \\
\hline 5 & 39 & Maling & \\
\hline 6 & 40 & Zawo & \\
\hline 7 & 41 & Schliebunder & \\
\hline 8 & 42 & Vorluch & \\
\hline 9 & 43 & verkraffern & \\
\hline 10 & 44 & sulibritzen & \\
\hline 11 & 45 & Fallurwakel & \\
\hline 12 & 46 & bestrugeln & \\
\hline 13 & 47 & verklubenatzt & \\
\hline 14 & 48 & Laukolz & \\
\hline 15 & 49 & Pfitzorkaden & \\
\hline 16 & 50 & resubelt & \\
\hline 17 & 51 & lefal & Külinge \\
\hline 18 & 52 & \\
\hline
\end{tabular}

\begin{tabular}{|l|l|l|l|}
\hline 19 & 53 & krauern & richtig? \\
\hline 20 & 54 & Blukenbarling & \\
\hline 21 & 55 & Naloß & \\
\hline 22 & 56 & sallen & \\
\hline 23 & 57 & plabeln & \\
\hline 24 & 58 & geschraten & \\
\hline 25 & 59 & Kattaus & \\
\hline 26 & 60 & gerutten & \\
\hline 27 & 61 & Mindinnen & \\
\hline 28 & 62 & Frielaume & \\
\hline 29 & 63 & Saberlicke & \\
\hline 30 & 64 & pfranden & \\
\hline 31 & 65 & karflumen & \\
\hline 32 & 66 & Jalosse & \\
\hline 33 & 67 & Kudilabert & \\
\hline 34 & 68 & verkrabaten & \\
\hline 35 & 69 & Quitensana & \\
\hline 36 & 70 & Arbaklaschen & \\
\hline GES & & \\
\hline & & \\
\hline
\end{tabular}




\section{FARBSTRICHE BENNENNEN}

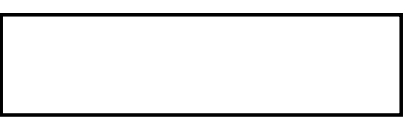

sek für eine Seite “Farbbalken Bennenen“ im Farbworte-Interferenz-Test (,STROOP“-Test)

\section{TRIGRAMME}

mit Probandennummer auf Kassette aufnehmen.

sek für $10 \mathrm{x}$ „Baum - Schuh - Topf“ 


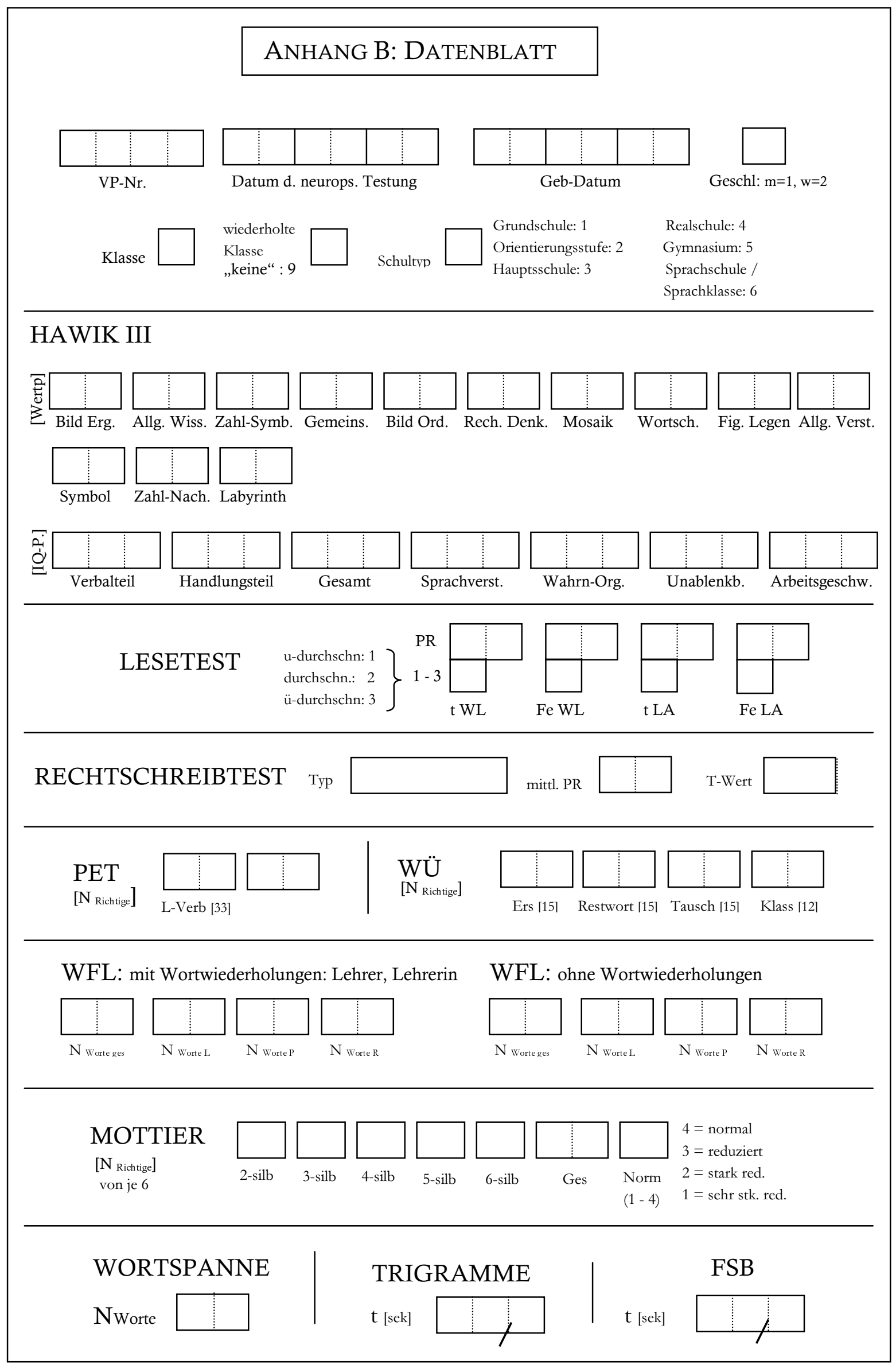




\section{LEGENDE ZUM DATENBLATT}

$\begin{array}{ll}\text { VP-Nr.: } & \text { Versuchspersonen-Nummer } \\ \text { Datum d. neurops. Testung: } & \text { Datum der neuropsychologischen Testung } \\ \text { Geschl: } & \text { Geschlecht } \\ \text { m / w } & \text { männlich / weiblich }\end{array}$

HAWIK-III

HAMBURG WECHSLER
INTELLIGENZTEST, 3 . AUSG.

Wertp.:

Bild. Erg.

$$
\text { Wertpunkte }
$$

Allg. Wiss.

Bilder Ergänzen

Zahl-Symb.

Allgemeines Wissen

Gemeins.

Zahlen-Symbol-Test

Bild Ord.

Gemeinsamkeiten Finden

Rech. Denk.

Bilder Ordnen

Wortsch.

Rechnerisches Denken

Fig.-Legen

Wortschatz-Test

Allg. Verst.

Figuren Legen

Zahl-Nach.

Allgmeines Verständnis

Zahlen Nachsprechen (gesamt)

IQ-P.:

IQ-Punkte

Sprachverst.

Sprachverständnis

Wahrn.-Org.

Wahrnehmungs-Organisation

Unablenkb.

Unablenkbarkeit

Arbeitsgeschw.

Arbeitsgeschwindigkeit

\section{LESETEST}

u-durchschn.:

unterdurchschnittlich

ü-durchschn.:

überdurchschnittlich

PR:

t LA:

Prozentrang

Fe LA:

Leseabschnitte: Zeitwert

Fe WL:

Leseabschnitte: Fehlerwert

tWL:
PET

L-Verb:

W-Erg:

WÜ TESTBATTERIE

Ers:

Restwort:

Tausch:

Klass:

WFL

N Worte ges / L / P / R:

\section{WORTFLÜSSIGKEITSTEST}

Anzahl gesamt / mit Anfangsbuchstaben L / $\mathrm{P}$ / R produzierter Worte

\section{MOTTIER}

\begin{tabular}{|c|c|}
\hline 2- bis 6-sil: & 2- bis 6-silbige Items \\
\hline red: & reduziert \\
\hline stark. red.: & stark reduziert \\
\hline FSB & FARBSTRICHE BENENNEN \\
\hline $\mathrm{t}:$ & Zeit \\
\hline
\end{tabular}




\section{ANHANG C: KOVARIANZ-/KORRELATIONSMATRIX}

\begin{tabular}{|c|c|c|c|c|c|c|c|c|c|c|c|}
\hline & & \multicolumn{3}{|c|}{ Phonologische Bewusstheit } & \multicolumn{3}{|c|}{ phonologisches Arbeitsgedächtnis } & \multirow{2}{*}{$\begin{array}{c}\text { Abruf des } \\
\text { LZG }\end{array}$} & \multicolumn{3}{|c|}{ EEG } \\
\hline & $\begin{array}{c}\text { Recht- } \\
\text { schrei-bung }\end{array}$ & $\begin{array}{c}\text { Laute } \\
\text { ersetzen } \\
\text { (Wzbg.) }\end{array}$ & $\begin{array}{c}\text { Laute } \\
\text { vertau- } \\
\text { schen } \\
\text { (Wzbg.) }\end{array}$ & $\begin{array}{l}\text { Laute } \\
\text { klassi- } \\
\text { fizieren } \\
\text { (Wzbg.) }\end{array}$ & Mottier & $\begin{array}{c}\text { Trigram- } \\
\text { me }\end{array}$ & $\begin{array}{l}\text { Kunst- } \\
\text { worte: } \\
\text { Inter- } \\
\text { aktion }\end{array}$ & & PHO & NP & SIN \\
\hline $\begin{array}{l}\text { Recht- } \\
\text { schreib }\end{array}$ & $\begin{array}{c}\mathbf{1 . 0 0 0} \\
218.631206\end{array}$ & & & & & & & & & & \\
\hline \begin{tabular}{|l} 
Laute \\
ersetzen
\end{tabular} & $\begin{array}{c}.735 \\
41.297872\end{array}$ & $\begin{array}{c}1.000 \\
16.170213\end{array}$ & & & & & & & & & \\
\hline $\begin{array}{l}\text { Laute } \\
\text { vertau- }\end{array}$ & $\begin{array}{c}.719 \\
.203093\end{array}$ & $\begin{array}{c}.595 \\
.047171\end{array}$ & $\begin{array}{c}1.000 \\
.000466\end{array}$ & & & & & & & & \\
\hline $\begin{array}{l}\text { Laute } \\
\text { klassifi- }\end{array}$ & $\begin{array}{c}.693 \\
.054301\end{array}$ & $\begin{array}{c}.702 \\
.006533\end{array}$ & $\begin{array}{c}.529 \\
.000013\end{array}$ & $\begin{array}{c}1.000 \\
.000217\end{array}$ & & & & & & & \\
\hline Mottier & $\begin{array}{c}.695 \\
39.737589\end{array}$ & $\begin{array}{c}.574 \\
8.446809\end{array}$ & $\begin{array}{c}.413 \\
.041528\end{array}$ & $\begin{array}{c}.643 \\
.008619\end{array}$ & $\begin{array}{c}1.000 \\
13.3758877\end{array}$ & & & & & & \\
\hline $\begin{array}{l}\text { Tri- } \\
\text { gramme }\end{array}$ & $\begin{array}{c}.636 \\
32.533688\end{array}$ & $\begin{array}{c}.526 \\
5.085106\end{array}$ & $\begin{array}{c}.441 \\
.029126\end{array}$ & $\begin{array}{c}.546 \\
.004665\end{array}$ & $\begin{array}{c}.543 \\
6.666667\end{array}$ & $\begin{array}{l}1.000 \\
9.371897\end{array}$ & & & & & \\
\hline $\begin{array}{l}\text { Kunst- } \\
\text { worte : }\end{array}$ & $\begin{array}{c}.249 \\
26.785461\end{array}$ & $\begin{array}{c}.160 \\
6.765957\end{array}$ & $\begin{array}{c}.103 \\
.030860\end{array}$ & $\begin{array}{c}.093 \\
.003584\end{array}$ & $\begin{array}{c}.110 \\
6.716312\end{array}$ & $\begin{array}{c}.042 \\
4.234486\end{array}$ & $\begin{array}{c}1.000 \\
6.839982\end{array}$ & & & & \\
\hline $\begin{array}{l}\text { Wort- } \\
\text { produk- }\end{array}$ & $\begin{array}{c}.533 \\
52.258865\end{array}$ & $\begin{array}{c}.610 \\
12.191489\end{array}$ & $\begin{array}{c}.514 \\
.056665\end{array}$ & $\begin{array}{c}.641 \\
.013415\end{array}$ & $\begin{array}{c}.458 \\
14.780142\end{array}$ & $\begin{array}{c}.396 \\
10.419326\end{array}$ & $\begin{array}{c}.137 \\
11.112589\end{array}$ & $\begin{array}{c}1.000 \\
43.913121\end{array}$ & & & \\
\hline PHO & $\begin{array}{c}.271 \\
.001859\end{array}$ & $\begin{array}{c}.179 \\
.000421\end{array}$ & $\begin{array}{c}.181 \\
.000001\end{array}$ & $\begin{array}{c}-.069 \\
.000001\end{array}$ & $\begin{array}{c}.226 \\
.000304\end{array}$ & $\begin{array}{c}.147 \\
.000257\end{array}$ & $\begin{array}{c}.127 \\
-.000084\end{array}$ & $\begin{array}{c}.161 \\
.000494\end{array}$ & $\begin{array}{c}1.000 \\
.000002\end{array}$ & & \\
\hline NP & $\begin{array}{c}.324 \\
13.192786\end{array}$ & $\begin{array}{c}.175 \\
2.807364\end{array}$ & $\begin{array}{c}.250 \\
.018425\end{array}$ & $\begin{array}{c}.234 \\
.009669\end{array}$ & $\begin{array}{c}.253 \\
1.0766306\end{array}$ & $\begin{array}{c}.309 \\
2.111864\end{array}$ & $\begin{array}{c}.238 \\
1.686413\end{array}$ & $\begin{array}{c}.096 \\
1.750693\end{array}$ & $\begin{array}{c}-.022 \\
-.000028\end{array}$ & $\begin{array}{c}1.000 \\
7.605607\end{array}$ & \\
\hline SIN & $\begin{array}{c}.340 \\
14.833281\end{array}$ & $\begin{array}{c}.270 \\
2.413985\end{array}$ & $\begin{array}{c}.211 \\
.015183\end{array}$ & $\begin{array}{c}.302 \\
.004631\end{array}$ & $\begin{array}{c}.203 \\
2.916303\end{array}$ & $\begin{array}{c}.238 \\
1.903644\end{array}$ & $\begin{array}{c}.106 \\
2.331568\end{array}$ & $\begin{array}{c}.228 \\
4.450069\end{array}$ & $\begin{array}{c}-.135 \\
-.000185\end{array}$ & $\begin{array}{c}.205 \\
1.671010\end{array}$ & $\begin{array}{c}1.000 \\
8.710263\end{array}$ \\
\hline \multicolumn{12}{|c|}{$\begin{array}{l}\text { Korrelations- und Kovarianzmatrix der pro Verarbeitungsebene am stärksten Rechtschreibvarianz erklärenden } \\
\text { Variablen und der Rechtschreibung }\end{array}$} \\
\hline $\begin{array}{l}\text { Wzbg.: } \\
\text { PHO: } \\
\text { NP: } \\
\text { SIN: }\end{array}$ & $\begin{array}{l}\text { Test entsta } \\
\text { Peaklatenz } \\
\text { Fläche des } \\
\text { Fläche der }\end{array}$ & $\begin{array}{l}\text { nmt der Wi } \\
\text { des zweiten } \\
\text { zweiten Seg } \\
\text { Sinusbeding }\end{array}$ & $\begin{array}{l}\text { zzburger T } \\
\text { Segments } \\
\text { ants der } 1 \\
\text { ang SIN-D }\end{array}$ & $\begin{array}{l}\text { tbatterie } \\
\text { r phonolo } \\
\text { htphonol }\end{array}$ & $\begin{array}{l}\text { schen Beding } \\
\text { ischen Bedin }\end{array}$ & $\begin{array}{l}\text { ung PH-G } \\
\text { gung NP-D }\end{array}$ & & & & & \\
\hline
\end{tabular}

Die obige Kovarianzmatrix bildete die Basis der LISREL-Modelle. Aufgrund derer besseren Lesbarkeit wurde in die Tabelle auch die Korrelationsmatrix integriert. Die Kovarianzwerte wurden mit sechs Dezimalstellen in die LISREL-Syntax eingelesen, so dass auf keiner Position ein Nullwert blieb. 


\section{DANKSAGUNGEN}

Hiermit möchte ich allen meinen Dank aussprechen, die zum Gelingen dieser Arbeit beigetragen haben.

- An erster Stelle steht dabei Herr Prof. Dr. A. Rothenberger, der mir die Möglichkeit eröffnet hat, dieses Thema zu bearbeiten.

- Ebenso gilt mein herzlicher Dank Herrn Prof. Dr. M. Hasselhorn. Sein „Hintergrunddienst“ zu Anfang wandelte sich während der langen Auswertungsphase zu einer mitunter recht zeitintensiven Betreuung, während der es ihm immer wieder gelang, innerhalb kürzester Zeit die wichtigsten Essenzen herauszudestillieren und die wichtigsten Impulse zu setzen. Ohne seine Unterstützung wäre diese Arbeit nicht möglich gewesen.

- Ein herzliches Dankeschön möchte ich der Konrad-Adenauer-Stiftung, insbesondere Frau Dr. Daniela Tandecki, aussprechen. Die Stiftung ermöglichte die Dissertation nicht nur durch ihre dreijährige finanzielle Unterstützung. Ebenso profitierte ich von den persönlichen Kontakten sowie von den jeweils exzellenten Seminaren.

- Herr Dr. T. Banaschewski war verantwortlich für die Leitung des Projekts, in dessen Kontext die vorliegende Dissertation entstand. Er war bei aktuellen Belangen gefordert.

- Herr Dr. W. Woerner partizipierte die Leitungsfunktion Herrn Dr. T. Banaschewskis, indem er das Voranschreiten des Projekts mit aufmerksamem Blick beobachtete. So mancher nichtbegangene Fehler ist seiner Wachsamkeit zuzuschreiben. Mit seiner Einarbeitung meiner Person in die Syntax-Funktion von SPSS erwies er mir weiterhin besonders für die Auswertungsphase einen unschätzbaren Dienst.

- Susanne Mock war die technische Assistentin des Projekts. Ohne ihren immensen zeitlichen Beitrag wäre die Durchführung niemals möglich gewesen.

- Bjoern Albrecht arbeitete sich in die Verarbeitung von EEG-Daten ein. Ihm danke ich insbesondere für seine immer unkomplizierte und frische Zusammenarbeit.

- Andreas Becker, Judith Bönnighausen, Julia Dreesen und Martina Noé danke ich für die Mithilfe bei der Untersuchung der Kinder. 
- Immer wieder mit Fragen von meiner Seite konfrontiert sah sich Frau Priv.Doz. Dr. Micha Strack, und war jeweils um eine fundierte Antwort bemüht. Desgleichen erklärte sich Michael Bast spontan bereit, mich in die Funktionsweise des Programmes LISREL einzuweisen.

- Die Therapeuten der Klinder-/Jugendpsychiatrie sowie den niedergelassenen Therapeuten, die zwischen dem Projekt und ihren Patienten einen Kontakt herstellten, sei an dieser Stelle ein aufrichtiger Dank ausgesprochen.

- Mascha Rieke, geb. Schallenberg, vermittelte in ihrer Funktion als Lehrerin zwischen der Grundschule am Harz in Hattorf und dem Projekt und verhalf der Studie dadurch zu einem rascheren Untersuchen der notwendigen Anzahl an Kindern.

- Ich möchte außerdem ein herzliches Dankeschön an meine Familie und an meine Freunde richten, die mich während der langen Zeit liebevoll unterstützt haben. 


\title{
CURRICULUM VITAE
}

\author{
geboren: \\ 15.02 .1972 \\ Geburtsort: \\ Nürnberg / Deutschland \\ Staatsangehörigkeit: deutsch \\ $1978-1991 \quad$ Abitur in Oberasbach bei Nürnberg. \\ 1991 - $1998 \quad$ Studium der Biologie in Erlangen, Genf und Göttingen. \\ Schwerpunkte: Biochemie, Pharmazie, Botanik. \\ Diplomarbeit: Wirkung eines Conustoxins auf Ionenkanäle des Gehirns und des \\ Muskels. \\ $1997-2002$ \\ Studium der Psychologie in Göttingen. \\ Schwerpunkte: Pädagogische Psychologie; Klinische Psychologie. \\ Diplomarbeit: Zentrale Exekutive des Arbeitsgedächtnis-Modells nach Baddeley. \\ 2000 - $2004 \quad$ Promotion in der Abteilung für Enwicklungspsychologie am Georg-Elias- \\ Müller-Institut für Psychologie Göttingen und in Kooperation mit der Abtei- \\ lung für Kinder- und Jugendpsychiatrie des Klinikums Göttingen. \\ Förderung durch ein Promotionsstipendium der Konrad-Adenauer-Stiftung.
}

